\title{
Hanford Wells
}

March 1976

Prepared for the U.S. Energy

Research and Development Administration under Contract E(45-1):1830 


\section{DISCLAIMER}

This report was prepared as an account of work sponsored by an agency of the United States Government. Neither the United States Government nor any agency Thereof, nor any of their employees, makes any warranty, express or implied, or assumes any legal liability or responsibility for the accuracy, completeness, or usefulness of any information, apparatus, product, or process disclosed, or represents that its use would not infringe privately owned rights. Reference herein to any specific commercial product, process, or service by trade name, trademark, manufacturer, or otherwise does not necessarily constitute or imply its endorsement, recommendation, or favoring by the United States Government or any agency thereof. The views and opinions of authors expressed herein do not necessarily state or reflect those of the United States Government or any agency thereof. 


\section{DISCLAIMER}

Portions of this document may be illegible in electronic image products. Images are produced from the best available original document. 
NOTICE

This report was prepared as an account of work sponsored by the United States Government. Neither the United States nor the United States Energy Research and Development Administration, nor any of their employees, nor any of their contractors, subcontractors, or their employees, makes any warranty, express or implied, or assumes any legal liability or responsibility for the accuracy, completeness or usefulness of any information, apparatus, product or process disclosed, or represents that its use would not infringe privately owned rights.

PACIFIC NORTHWEST LABORATORY

operated by

BATTELLE

for the

U.S. ENERGY RESEARCH AND DEVELOPMENT ADMINISTRATION

Under Contract E(45-1)-1830

Printed in the United States of America

Available from

National Technical Information Service

U.S. Department of Commerce

5285 Port Royal Road

Springfield, Virginia 22151

Price: Printed Copy $\$ 5.00$; Microfiche $\$ 2.25$ 


\section{HANFORD WELLS}

\section{by}

V. L. McGhan, D. A. Myers and D. W. Damschen

\section{Resources Systems Section}

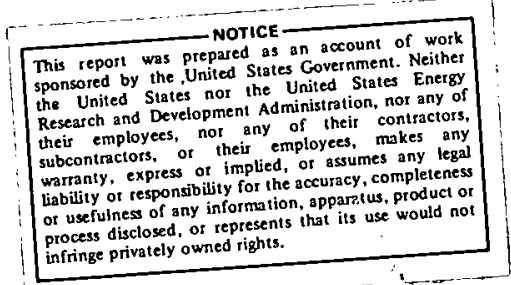

March 1976

\section{BATTELLE}

PACIFIC NORTHWEST LABORATORIES

RICHLAND, WASHINGTON 99352 
HANFORD WELLS

The Hanford Reservation contains about 2100 wells constructed from preHanford Works days to the present. More than 200 older wells were mostly hand-dug farm wells, many of which are no longer usable or even visible. The newer wells are cased wells which were either churn-drilled, drive-barrel-drilled or rotary-drilled. The wells drilled within the Hanford Reservation were constructed by contract drillers, by drilling crews in the employ of ERDA prime contractors, or by the U. S. Geological Survey. Some of these were constructed originally to provide sanitary water for outlying installations such as U.S. Army facilities, others to permit surveillance of the groundwater and sub-surface formations in the vicinity of waste disposal facilities, and still others to obtain data for a geological survey of the region. Some of these wells have been destroyed during construction of new facilities or through casing failures but are included on a master record to identify data obtained from them. As of January, 1976, about 1800 wells still exist. (Table 1 contains a summary of status.) About 850 of these existing wells were drilled to the groundwater table. About 700 of these wells (including about 20 farm wells) still contain water. The others have become dry through infiltration of sediments or a general lowering of the water table in their vicinity.

This report providing the most complete documentation of wells in and adjacent to the Hanford Reservation, supersedes all previous compilations of Hanford wells, the most recent being dated June 1973 (BNWL-1739). Not included in this report or the master record are those destroyed fram wells which were never surveyed into the Hanford coordinate system nor used for water table measurements or water sample collection. References 1,2 and 3 contain the available data on these wells.

\section{WELL NUMBERING SYSTEM}

The well structures are identified by a number composed of three basic parts in the form $X X X-X X X-X X X$. The first part is the facility number $X 99$, where $X$ is $1,2,3,6,11$, or 30 , depending on the Hanford Reservation area in which the well is located. The second and third groups of numbers vary by areas. 


\section{TABLE 1. HANFORD WELLS}

Wells Used For:

Groundwater Contamination Surveillance

266

Groundwater Hydrological Data Collection 323

Dry Wells for Monitoring Waste Management Facilities

980

Basalt Stratigraphy Characterization

14

Total Wells by Area:

$\begin{array}{lcc} & \text { Drilled } & \text { Existing } \\ 100 \text { Area } & 102 & 67 \\ 200 \text { Area } & 1213 & 1165 \\ 300 \text { Area } & 30 & 23 \\ 600 \text { Area } & 642 & 507 \\ 1100 \text { Area (Richland) } & 105 & 16(1961) \\ 3000 \text { Area (N. Richland) } & 8 & 6 \\ \text { Existing Total Wells by Site } \cdots & 2100 & 1783\end{array}$


Well numbers in the 100 Areas (reactor areas) are prefixed by 199. The second group of numbers refers to the area and to the sheet map for the portion of the area where that well is located. The letters B,D,K,F,H,N designate the specific 100 Area, and the number(s) in the group refer to the appropriate sheet map. Therefore, a well on sheet 3 of the 100-B Area would be numbered 199-B3-X. The sheet maps are shown on the official maps for an area. The 100-K and 100- $\mathrm{N}$ Areas were not divided into sheet maps so only the letter symbol appears for wells in these areas.

The third group of numbers refers to the well number within the sheet map area. In some cases wells have been assigned a 100 Area number even though they are outside the area fence. These wells monitor liquid waste ground disposal facilities outside the area fence but are associated with that particular area.

\section{AREAS}

We11 numbers in the 200 Area (Chemical Separations Area) are prefixed by 299, and the second group of numbers refers to the 200 East Area (E) or the 200 West Area ( $W$ ), followed by the sheet number as described above. The third group of numbers refers to the well number within the sheet map subarea. Some of the monitoring wells in the 200 Areas are dry wells (i.e., they do not extend to the water table). These have been differentiated from the water-table wells by making the third group of numbers greater than 50 .

\section{AREA}

Well numbers in the 300 Area are prefixed by 399. Designation of wells in the 300 Area (Laboratory Area) is identical to that described for the 100 and 200 Areas, but no area letter designation is used with the sheet map number in the second aroup of numbers.

\section{AREA}

The 600 Area includes all of the Hanford Reservation outside the limited access areas. Well numbers in this area are prefixed by 699. The second and third groups of numbers for 600 Area wells consist of the north and west 
plant coordinates, respectively, rounded off to the nearest 1000 feet. For example, a wel1-located at plant coordinates N25663, W14554 would be designated 699-26-15. If the well is located south or east of the plant coordinate origin, an $S$ or $E$ is used with the appropriate number. Some 600 Area wells are located with 1000 feet of each other; these have letters ( $, B, C$, etc.) following the numbers for unique identification". A few wells located outside of the Hanford Reservation boundaries have also been given 600 Area designations:

HANFORD TOWNSITE

The weils in the former Hanford townsite are designated as 600 Area wells with HAN as the second number group and the well number as the third number group. These wells were originally surveyed into the Hanford townsite grid which.was established during reactor construction. A conversion to plant coordinates has been made for this edition of the Hanford Wells book.

1100 and 3000 AREAS

We11 numbers in the 1100 Area (City of Richland) and the 3000 Area (old North Richland) have 1199 and 3099 prefixes, respectively. The second and third number groups are the Richland coordinates rounded to the nearest 1000 feet. These wells were located in the Richland coordinate system because the plant grid had not been extended this far south when they were surveyed. New wells within the Hanford Reservation boundaries in the 1100 and 3000 Areas are located and named in the 600 Area plant grid system. However, wells drilled since 1961 within the Richland city limits are not included in the listing.

\section{PIEZOMETER TUBES}

Piezometer tubes were installed in 85 cased wells to measure potentials and sample the aquifer at various depths. Presence of these tubes is indicated by a letter $(0, P, Q, R, S$, or $T)$ after the well number; these tubes are 1 isted with the corresponding well in this tabulation. "Six other sets of piezometer tubes are individually drilled several feet apart, and are also designated by the sequence of letters $P, Q, R$, etc. Each tube of this type is regarded as a separate well in the tabutation. 
WELL DATA

A tabulation of existing wells and others for which data are recorded is composed of sections, each of which reports wells from one of the areas. It was prepared from computer listings of the master well information file maintained by Battelle-Northwest Resources Systems Section.

\section{TABLE FORMAT}

Column 1 - Well Designation and EMA Number

A11 of the existing wells that can be located and others for which data have been reported are designated in Column 1 by the system described earlier. Well designations can change when wells are surveyed into a coordinate system or when a resurvey is made.

The EMA (Environmental Monitoring Analysis Accumulation and Reporting) number in this column identifies the well for the computer program which processes sampling schedules and results. All wells assigned an EMA number have been or are presently in the routine groundwater sampling schedule.

A series of asterisks indicates that a well or wells has been removed from the active wells list. These wells may have been filled in, abandoned or otherwise destroyed.

A single asterisk indicates that the preceding well had piezometer tubes which have since been removed. In cases where only selected piezometer tubes were removed, they are noted by the single asterisk.

A master record of all wells and piezometer tube installations is maintained. Copies of this record may be examined at offices of Battelle-Northwest, Atlantic Richfield Hanford Company and the Energy Research and Development Administration in Richland, Washington.

Column 2 - Coordinates

Well coordinates are listed in Column 2 when this information is available. Plant coordinates are designed "P", Richland coordinates "R". For certain wells the coordinates have been estimated. 


\section{Column 3 - Elevation of Casing}

The elevation of the top of the well casing is reported in feet above mean sea level. Some values have been estimated.

\section{Column 4 - Drilled Depth}

The depth of the well, as determined at the time the well was completed, is listed in feet below the top of the casing.

Column 5 - Depth to Water

The depth from the top of the casing to the water table is listed in feet for wells in which this distance has been measured. These depths to water can flutuate over several feet, particularly for wells near the Columbia , River. Several old farm wells which are not currently being used for water, table elevation measurements are designated "wet" or "dry" as appropriate. In addition numerous wells used by WPPSS as seismic shot holes are listed as "dry".

Column 6 - Casing Diameter

The diameter of the casing used in construction of the well is given in inches. A few of the wells constructed at Hanford required casing of more than one size, and for these only the largest diameter is recorded. The length of the shortest side is listed for rectangularly shaped farm wells.. Column 7 - Depth to Bottom of the Well

The most recent measurement of the bottom depth of the well is reported. Generally, wells become partially filled as sediments enter the well through the perforations. The depths shown in this column were determined from data obtained since 1974 .

Column 8 - Minimum and Maximum Depth to Perforations

The depths to the top and bottom of the well casing perforations are expressed in feet below the top of the well casing. Huwcver, in some wellis alternating perforated and unperforated sections may be between the depths listed. Wells that were completed with well screens have no perforation depths listed. The screen depths are listed under the comments column. 


\section{Column 9 - Date Completed}

This column records the date, to the nearest month where possible, on which the well. was completed. In some cases only the year in which the well was drilled is known.

Column 10 - Former Designations

Column 10 lists the designations that have been used to identify the well in the past. These include local Hanford names or names applied by the agency responsibie for constructing the well, such as the U.S.G.S. designations. The reference numbers used in this column refer to the reference listing for this report. The following is a list of other abbreviations used in the former designations column:

$\begin{array}{ll}\text { DH } & \text { Drill Hole } \\ \text { FFTF } & \text { Fast Flux Test Facility } \\ \text { HD } & \text { Hanford Durand (driller's name) } \\ \text { HI } & \text { Hanford International (driller's name) } \\ \text { HR } & \text { Hanford Ranney (driller's name) } \\ \text { N. Run } & \text { North Run (well taping and sampling route) } \\ \text { RD } & \text { Richland Durand } \\ \text { RI } & \text { Richland International } \\ \text { Rx MPy } & \text { Hanford Project Highway Route } x, \text { Milepost y. } \\ \text { RRY } & \text { Railroad yard } \\ \text { Sea. Inl. Emp. } & \text { Seattle Inland Empire Gas Co. } \\ \text { TH } & \text { Test Hole } \\ \text { USGS } & \text { U. S. Geological Survey } \\ \text { WBD } & \text { White Bluffs Durand } \\ \text { WPPSS } & \text { Washington Public Power Supply System } \\ \text { WWOG } & \text { Western Washington 0il and Gas Co. } \\ \times \text { W } & \text { Well number } x \\ \text { Iumn } 11-\text { Comments }\end{array}$

Noted in this column are: changes made to the wells during routine maintenance, sampling pump installation, seismic study wells, pumped water suppily wells, and wells which have had screens installed. Wells that were not located during the census of wells taken for this report are so indicated in this column. Probably many of these wells have been destroyed. 
While considerable effort has been made to obtain the most accurate and complete tabulation possible of the Hanford Reservation wells, inevitably some omissions and errors may exist, particularly for old farm wells. Periodic additions and corrections to this document will be issued to be made a part of this report.

Maps of the 200 East and 200 west Areas are included on the following pages, and a 600 Area map is in the binder. Only the wells which extend to ground-. water are shown in the 200 Area maps. The 600 Area map shows a 11 wells currently available for water level measurements and groundwater sampling. Area maps showing well locations are available for the 100-N Area $(\mathrm{H}-1-38646)$ and the 300 Area $(\mathrm{H}-3-32585)$. 


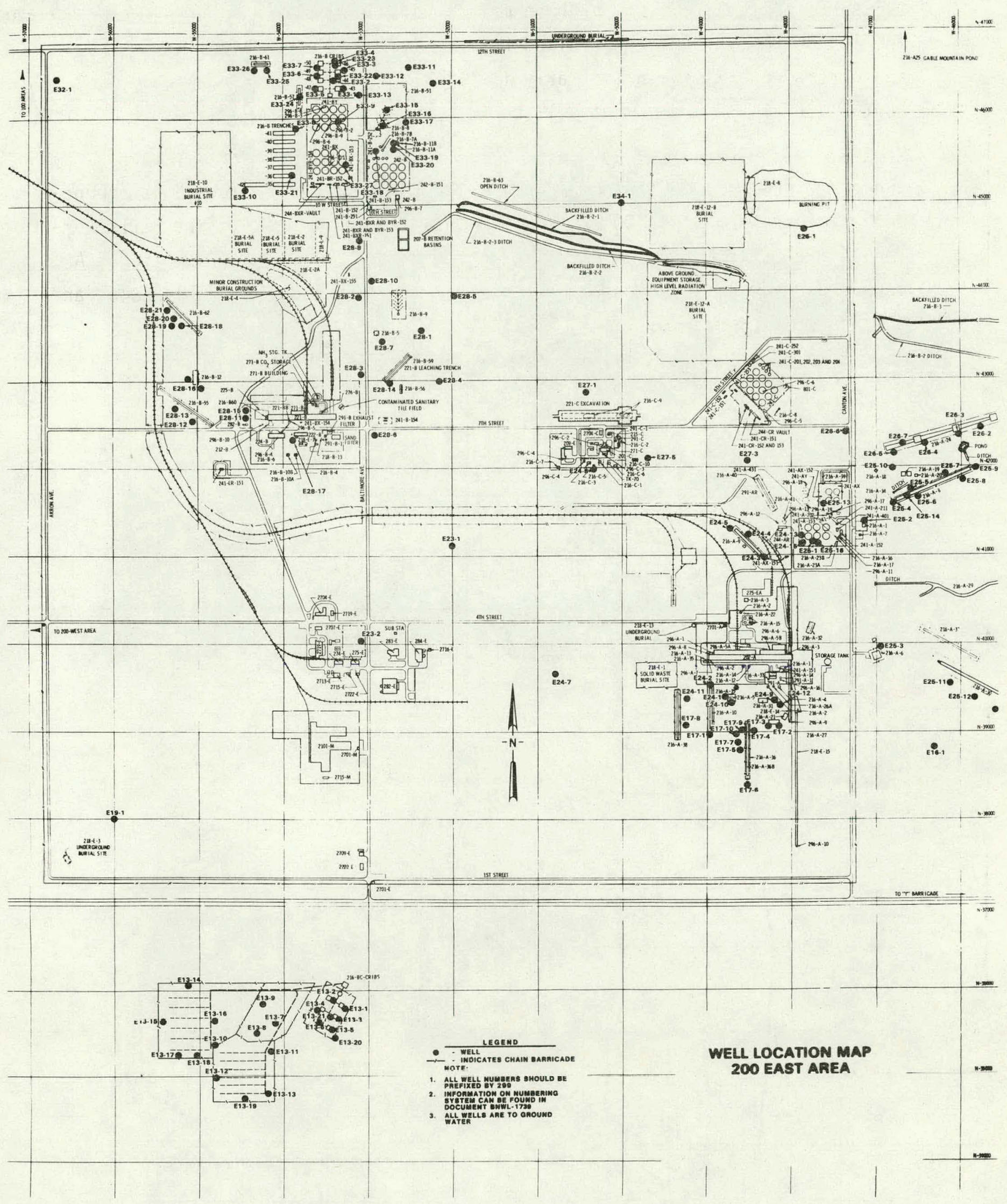




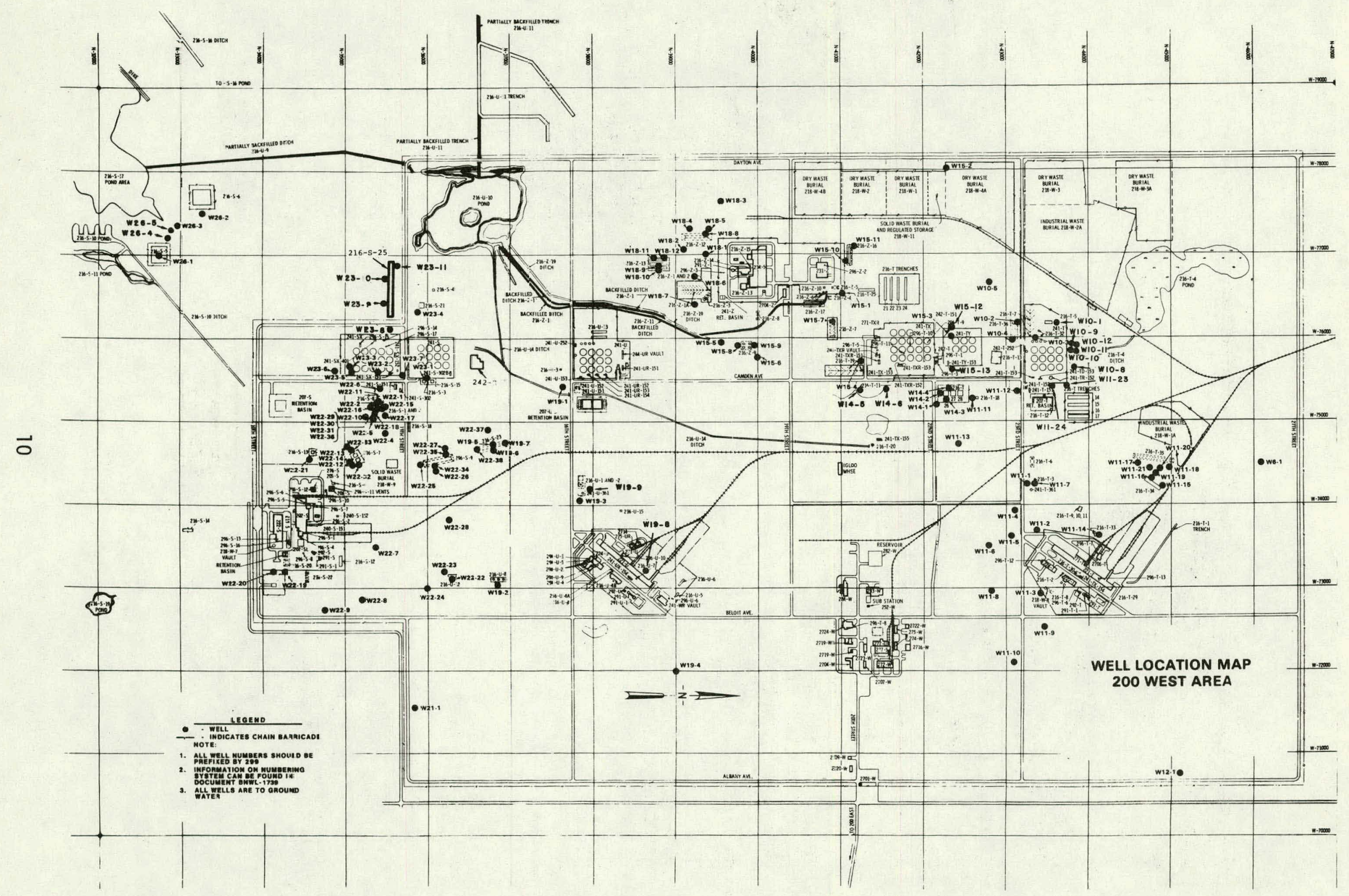

C. 


\section{REFERENCES}

1. Newcomb, R. C., and J. R. Strand, Geology and Groundwater. Characteristics of the Hanford Reservation of the Atomic Energy Commission, Washington. U.S.G.S. Interim Report No. $2,1953$.

2. Jenkins, 0. P. Underground Water Supply of the Region About White Bluffs and Hanford. Washington Department of Conservation and Development, Division of Geology, Bulletin No. 26, 1922.

3. Walters, K. L. and M. J. Grolier, Geology and Groundwater Resources of the Columbia Basin Project Area, Washington, State of Washington Water Supply BuTletin No. 8, Vo1. 1, 1960. 
WELL

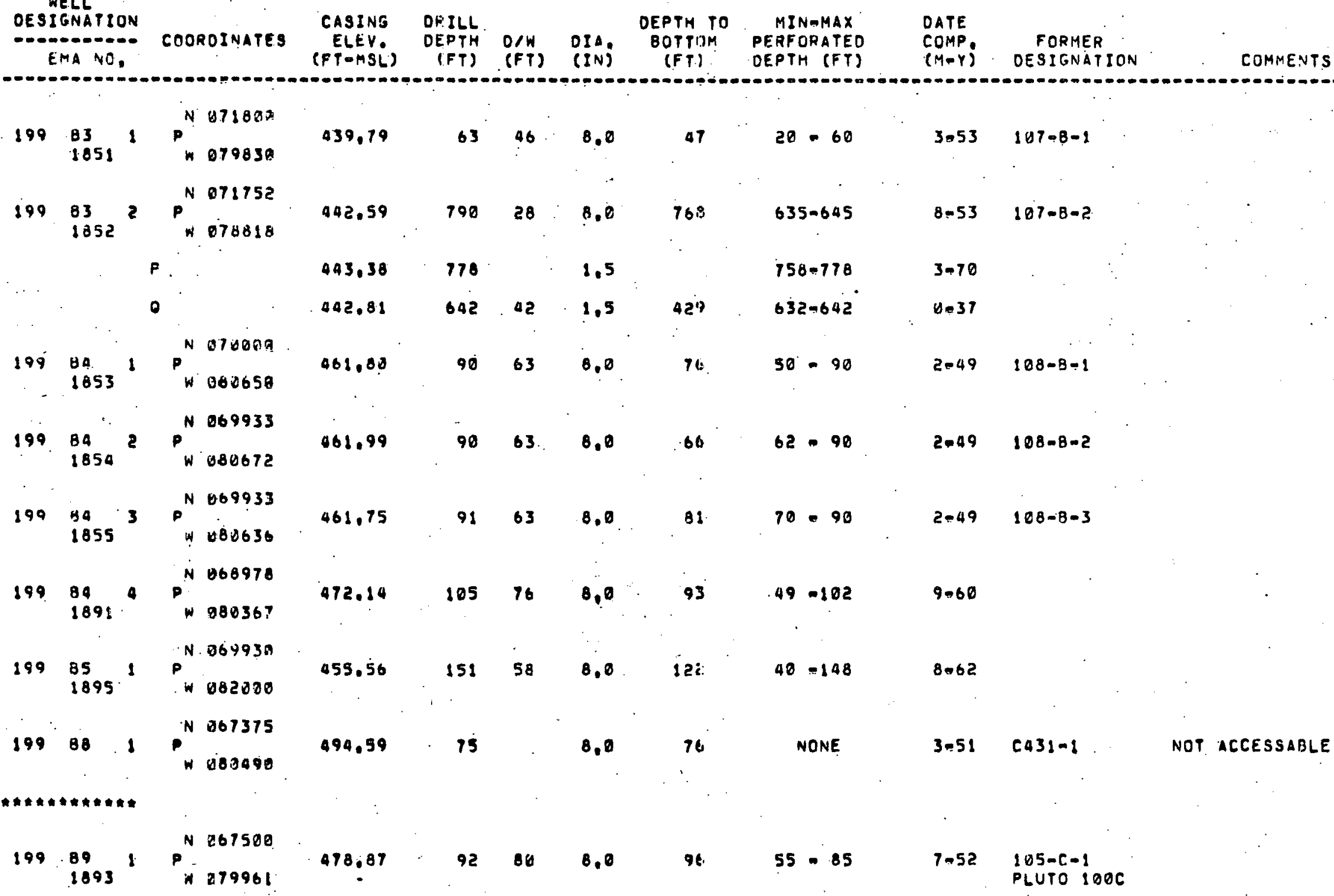


NELL

OESIGNA TION

COOROINATES

CASING DRILL

DRILL
DEP-H OIH OIA. BOTH TO MIN-MAX.

(FT) OEPTH (FT)

DATE

(M-Y)

FORMER

DESIGNATION

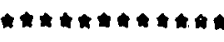

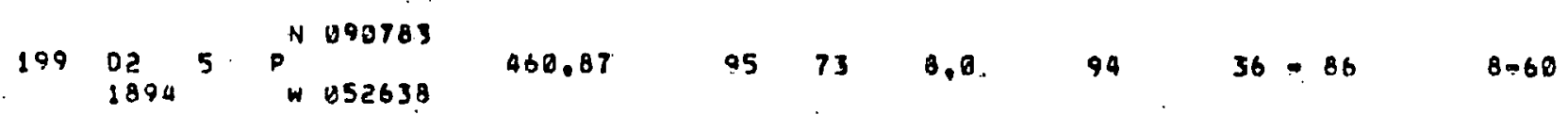

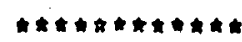

$199 \quad 05 \quad 10$

464.57

E'B ORY

8,0

29

$14 \cdot 27$.

$4057 \quad 120-0 R-1$

199 05. 18

464.57

27 DRY 8,0

28

$14: 27$

4-57 $100-D R-2$

$19905: 12 \quad P^{N} 092125$

4.69 .67

$9165 \quad 6.0$

91

$35 \cdot 90$

$8 \cdot 6 B$

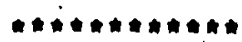

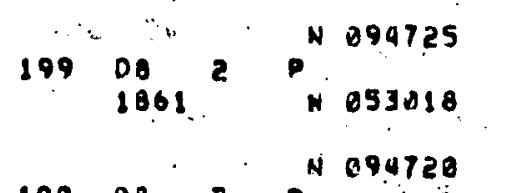

404.61

TT ORY

8.9

44

$30 \cdot 75$

$6-52^{\circ}, 107-0-1$

$199 \begin{array}{lll}08 & 3 & P^{N} 094728 \\ 1862 & & 0522 \mathrm{US}\end{array}$

499.06

8.1

$6,0 \quad 81$

$35 \cdot 79$

$6.52 \quad 107-0-2$ 


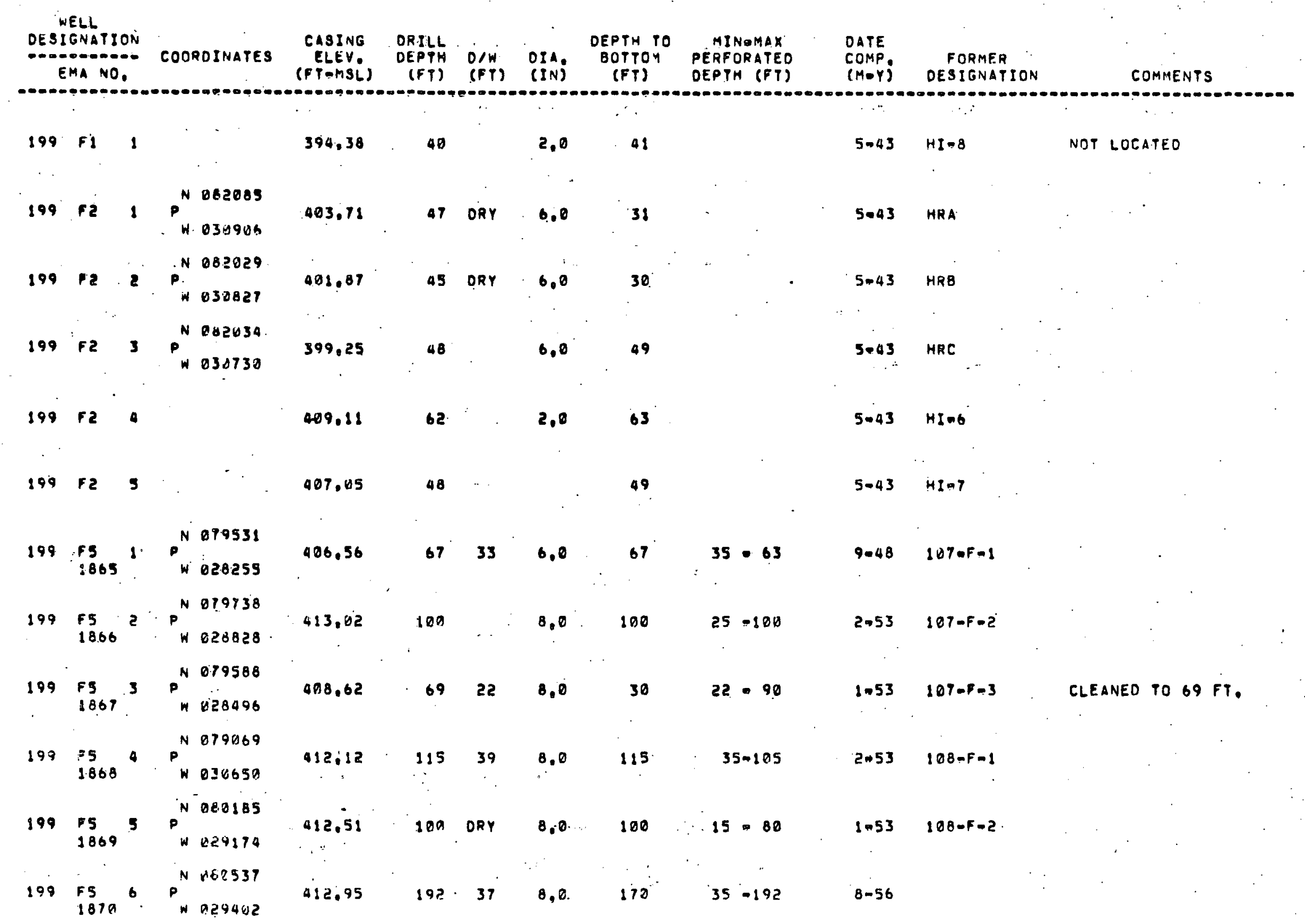


nELL

OESIGYATION

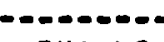

cooruinates

CASING DRILL

ELEV.

DEILTH O/W DIA: OEPTH TO
(FT) (FT) (IN)
(FT) $(F T \rightarrow M S L)$

EOTTOM
(FT)

MINAMAX
PERFORATEO PERFORATED
DEPTH (FT)

DATE

COMP.

DESIGNATION

COMMENTS

\section{N 179309}

199 F5 T P $P^{N 879309}$

412.76

39 ORY

6.8

28

4-58 ARMY ENG.

$19959 \quad 1 \quad P^{N 077199}$

339.74

$150 \quad 13 \quad 8.0$

121

10.140

$8-56$

$199+8 \therefore 1$ P. 075536

$\therefore 1860^{199} \quad 431265$

405.86

$57 \quad 34$

8.0

42

$13-53$.

$8 m 69$

$199 \quad F_{71} 2 p^{N 078661}$

490,74

55 DRY $8.0 \quad 22$

10.53

$8-60$

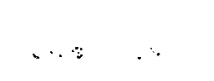

\section{" NELLL}

DESIGNATION

EMA: NO.

COQROINATES

CASING OKILL

ELEV. DEPTH D/W OIAD. BOTTOM if.Timsi)

(FT) (FT) (IN)

BOTTOM

MINMMAX
PERFORATED PERFORATED

OATE

COMP. FORMER

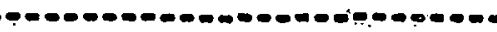

199 H3 1898

N. 094994 .

1898

$421: 98$

75. 45

3.0

75

$29 \cdot 89$

$8 \times 60$

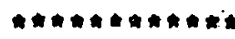

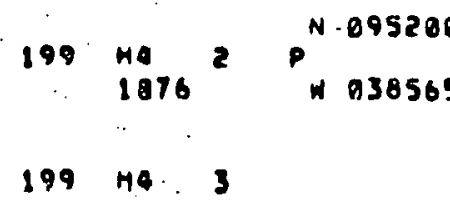

421.43

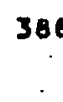

$\begin{array}{rrrr}386 & 3 & 0,0 & 319 \\ 55 & 39 & 0,0 & \therefore\end{array}$

311

5-52 $\quad 107-H \rightarrow 2$

CONFINED AQUIFER

19944.3

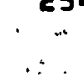

$34.55 . \quad 5074$

COMMENTS

DESIGNATION 


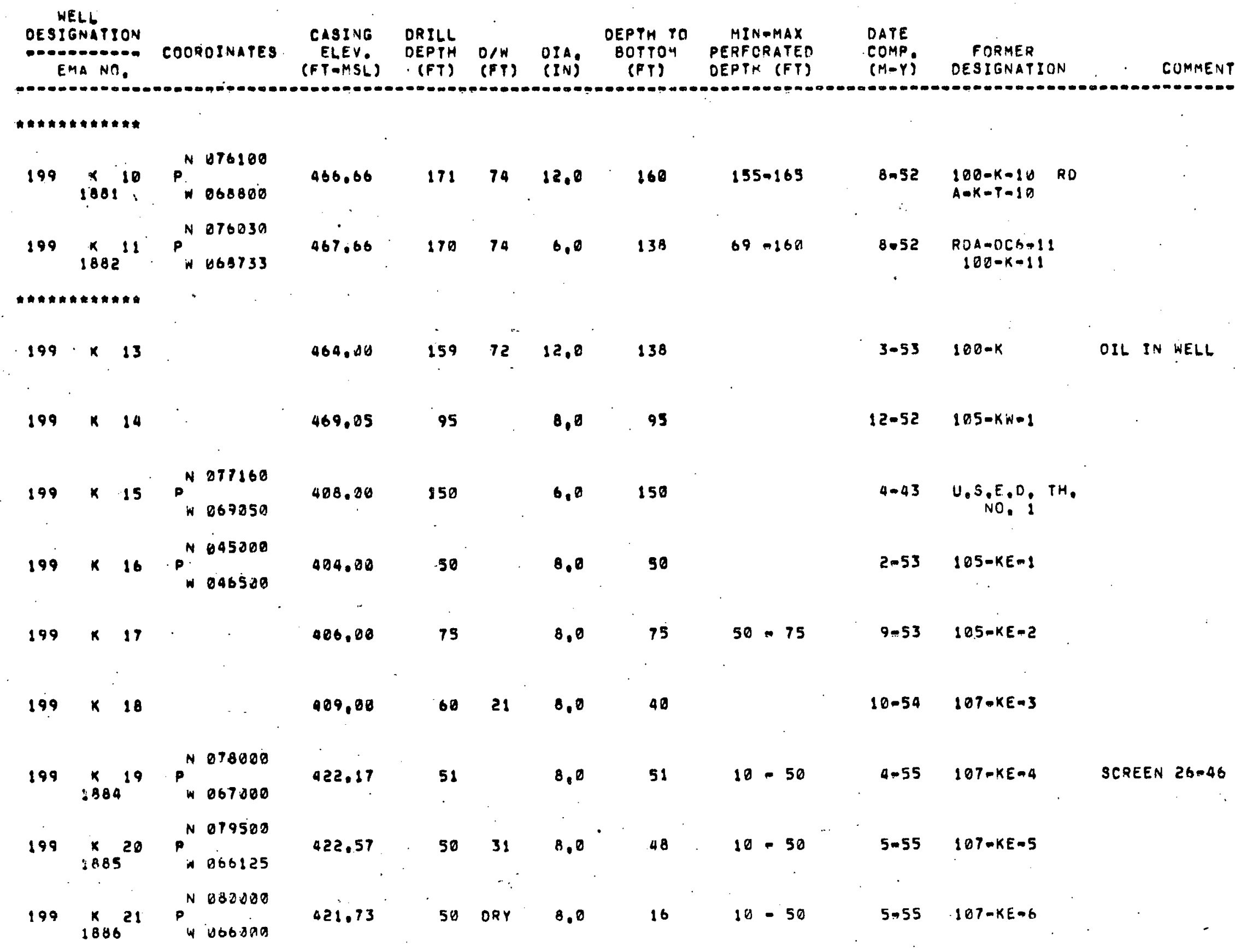


WELL

OESIGNATION

Domignation

COOROINATES

CASING DRILL

ELEV. UEPTH

o/n oIA

EPTH TO

MINTMAX

BOTPIS PERFORATEO

DATE

COMP. FORMER

(M-YY) DESISNATION

COMMENTS

\begin{tabular}{|c|c|c|c|c|c|c|c|c|c|c|c|c|c|c|c|}
\hline 199 & $\begin{array}{c}K \\
2.867\end{array}$ & 22 & $P_{W}^{N}$ & $\begin{array}{c}381070 \\
065000\end{array}$ & 421.68 & 50 & & 8,0 & 4.7 & $10 \cdot 50$ & 5.55 & $\begin{array}{c}107-K E-7 \\
:\end{array}$ & 425 & SCREEN & $23-49$ \\
\hline 199 & K & $\begin{array}{c}1 \\
23 \\
\vdots\end{array}$ & $P^{N}$ & $\begin{array}{l}078000 \\
068000\end{array}$ & 405.00 & 80 & DRY & 8.0 & 25 & $63 \cdot 00$ & $2-56$ & $1.706-K E R=1$ & & & \\
\hline 199 & $\mathbf{k}$ & 24 & $F^{N}$. & $\begin{array}{l}077430 \\
069000\end{array}$ & 467.28 & 50 & & 8.0 & 50 & : NONE & $12-52$ & $185-K W=Z$ & & . & . \\
\hline 199 & 2898 & 25 & $P^{N}$ & $\begin{array}{c}078000 \\
0 \\
068020\end{array}$ & $\begin{array}{r}4.73 .08 \\
-\quad:\end{array}$ & 76 & & 8.0 & 76 & $90=79$ & 8.53 & $105-K w-3$ & & & . \\
\hline 199 & K & 26 & . & $\therefore$ & 464.06 & 15 & & 0.0 & 15 & NONE & 8.53 & $115-K E-1$ & $\therefore$ & & \\
\hline
\end{tabular}




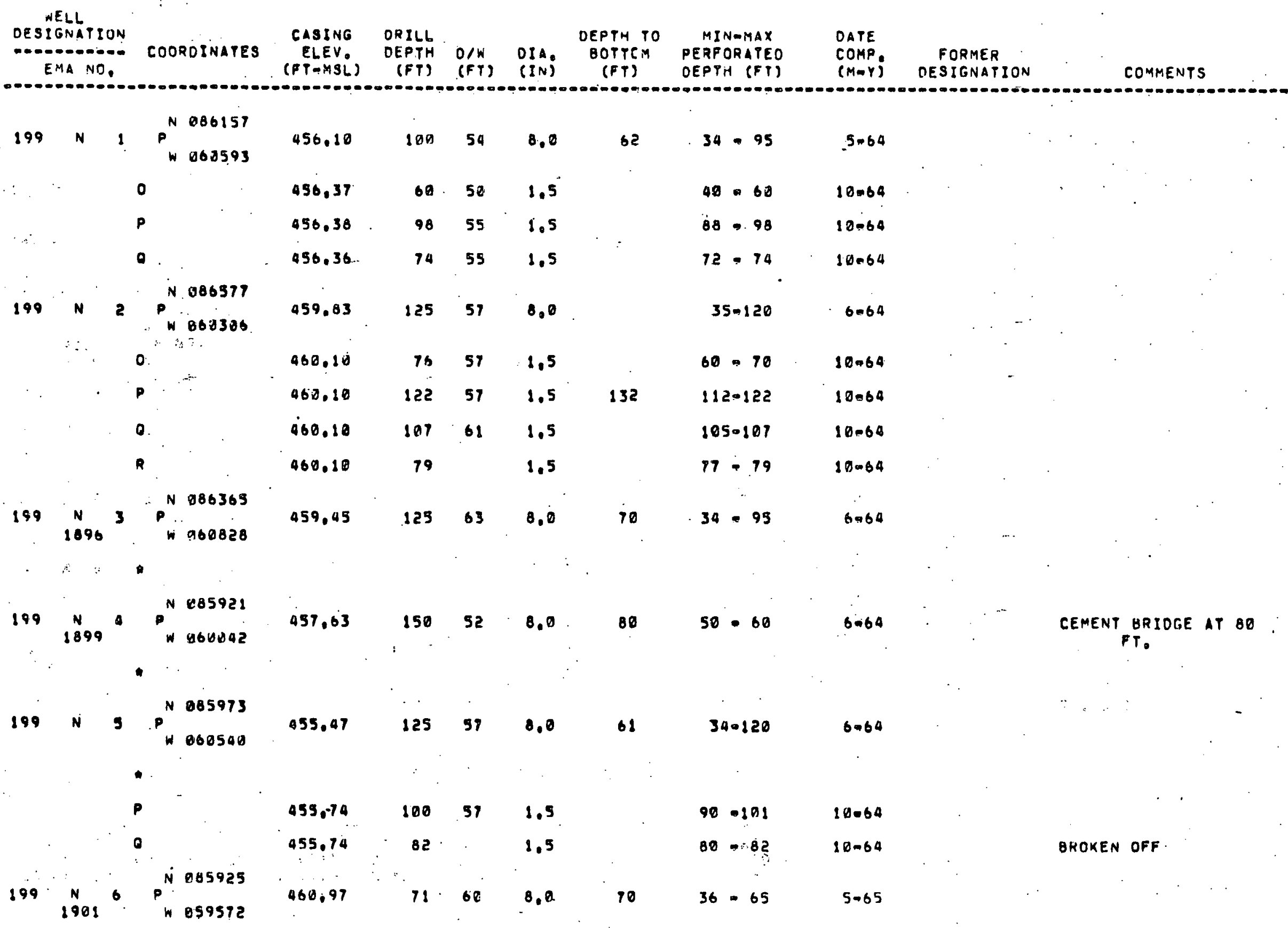


WELL.

OESIGNATION

EM NO

CoOROINATES

CASING DRILL

PERFORATEO

DEPTH (FT)

DATE

CUMP. FORMER

(M-Y) DESIGVATIUN

COMments

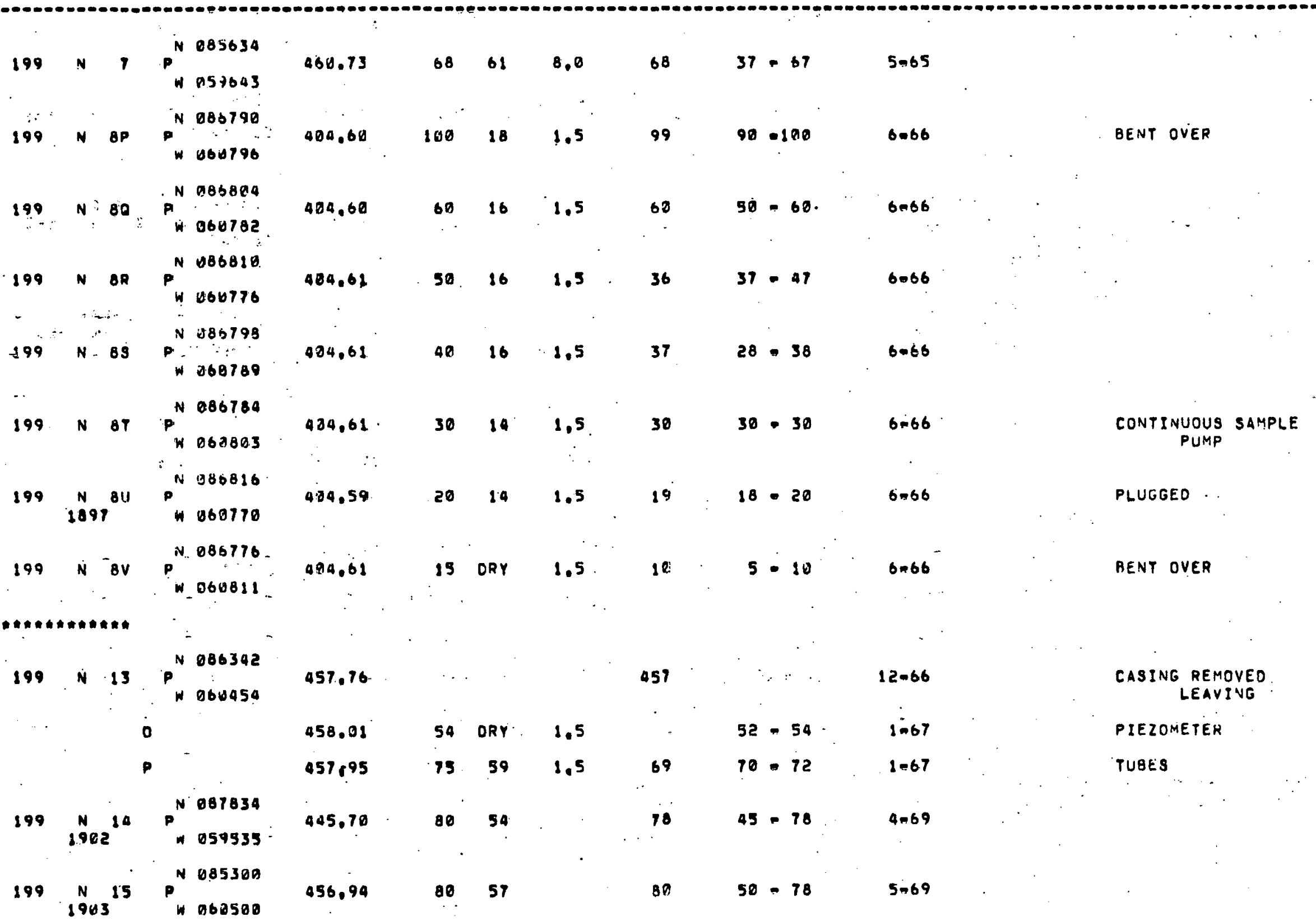


WELL

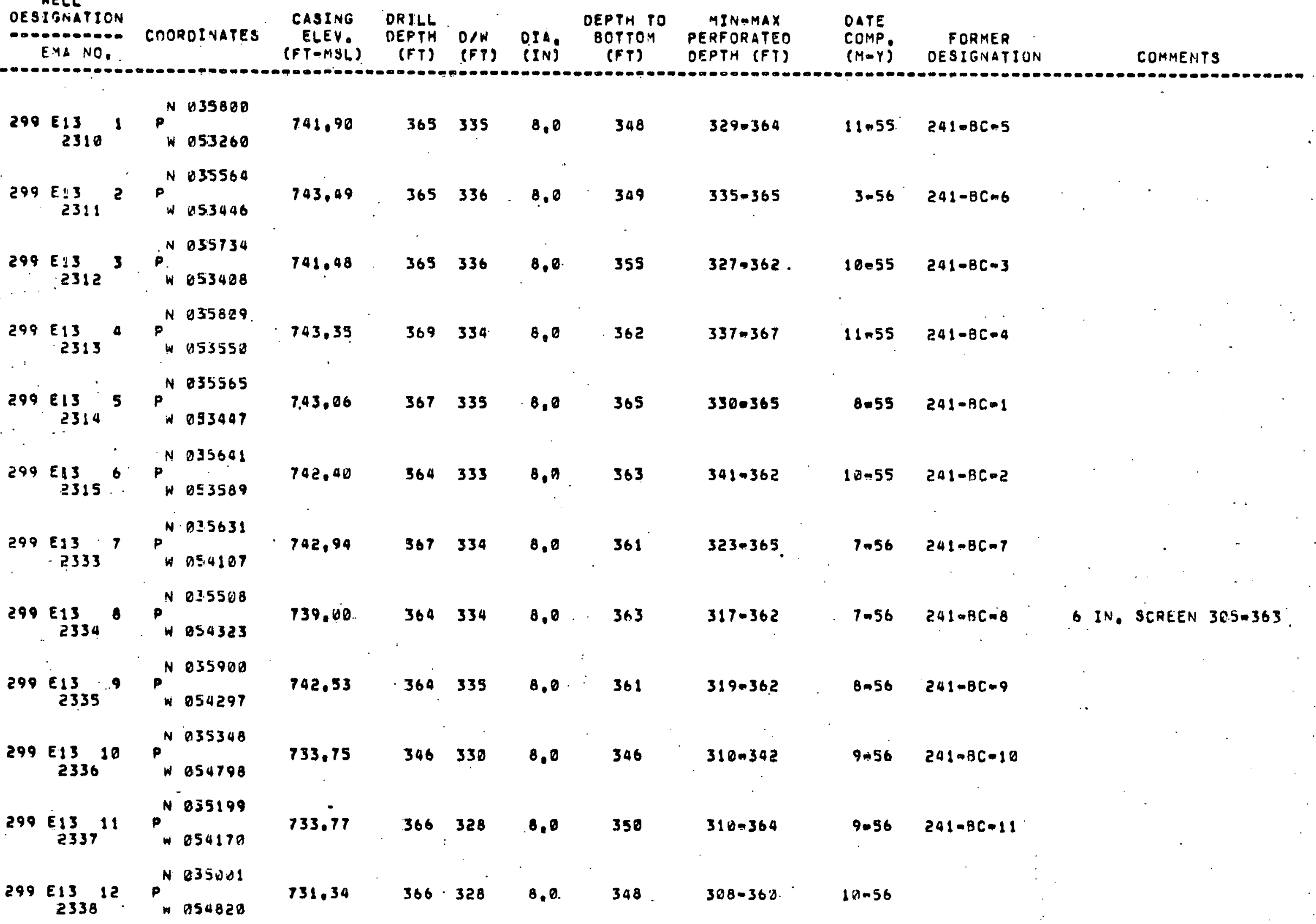


WELL

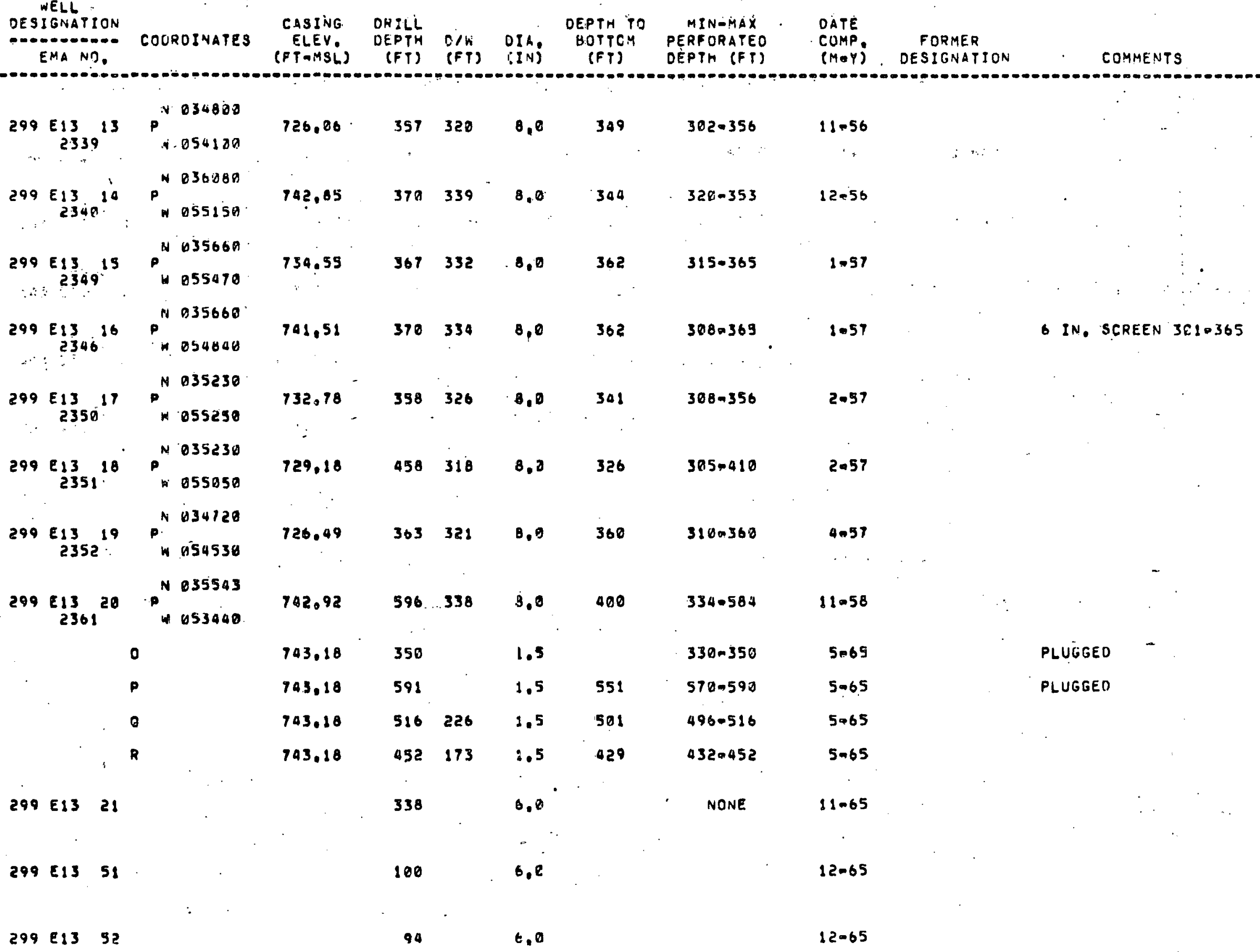




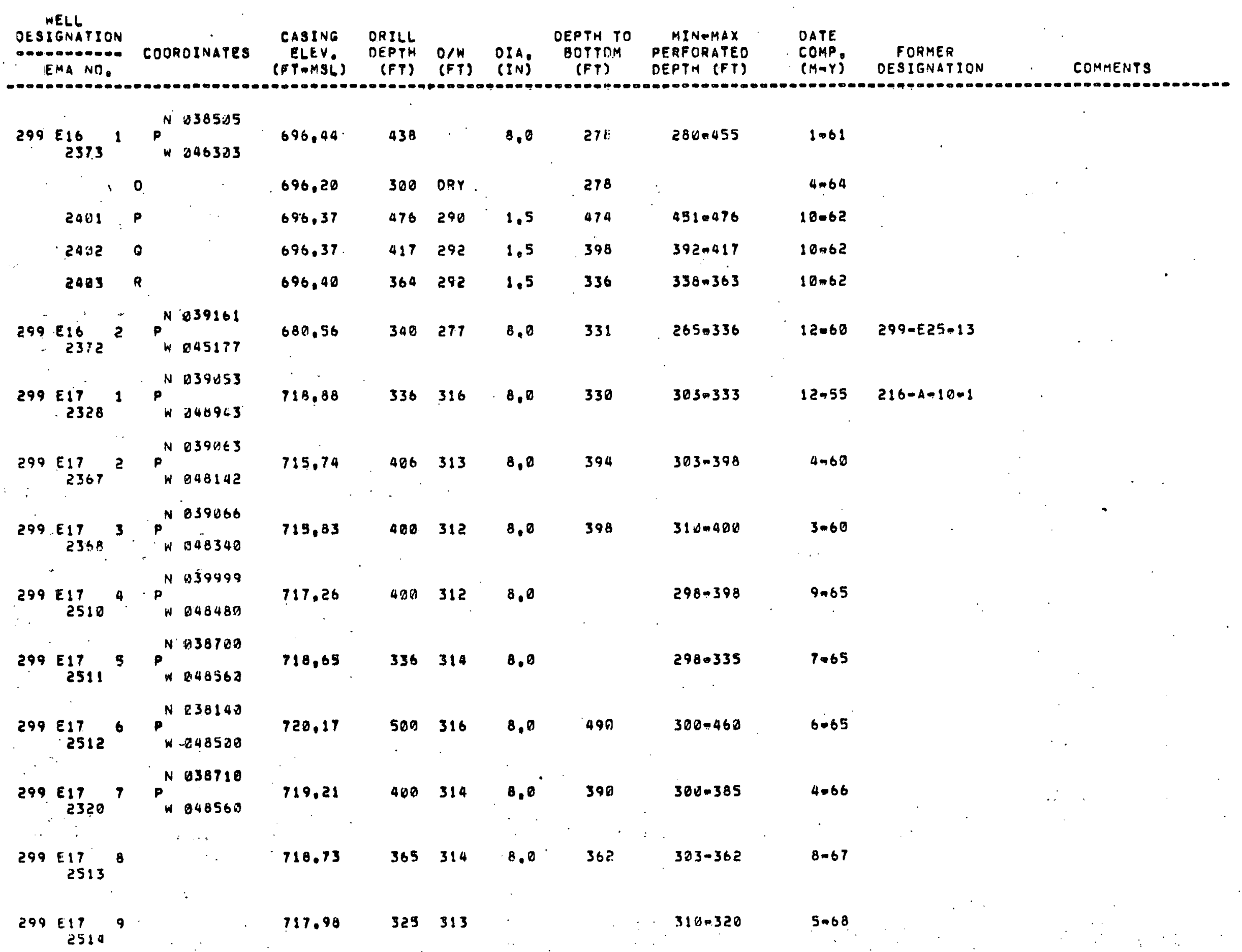


VEELL

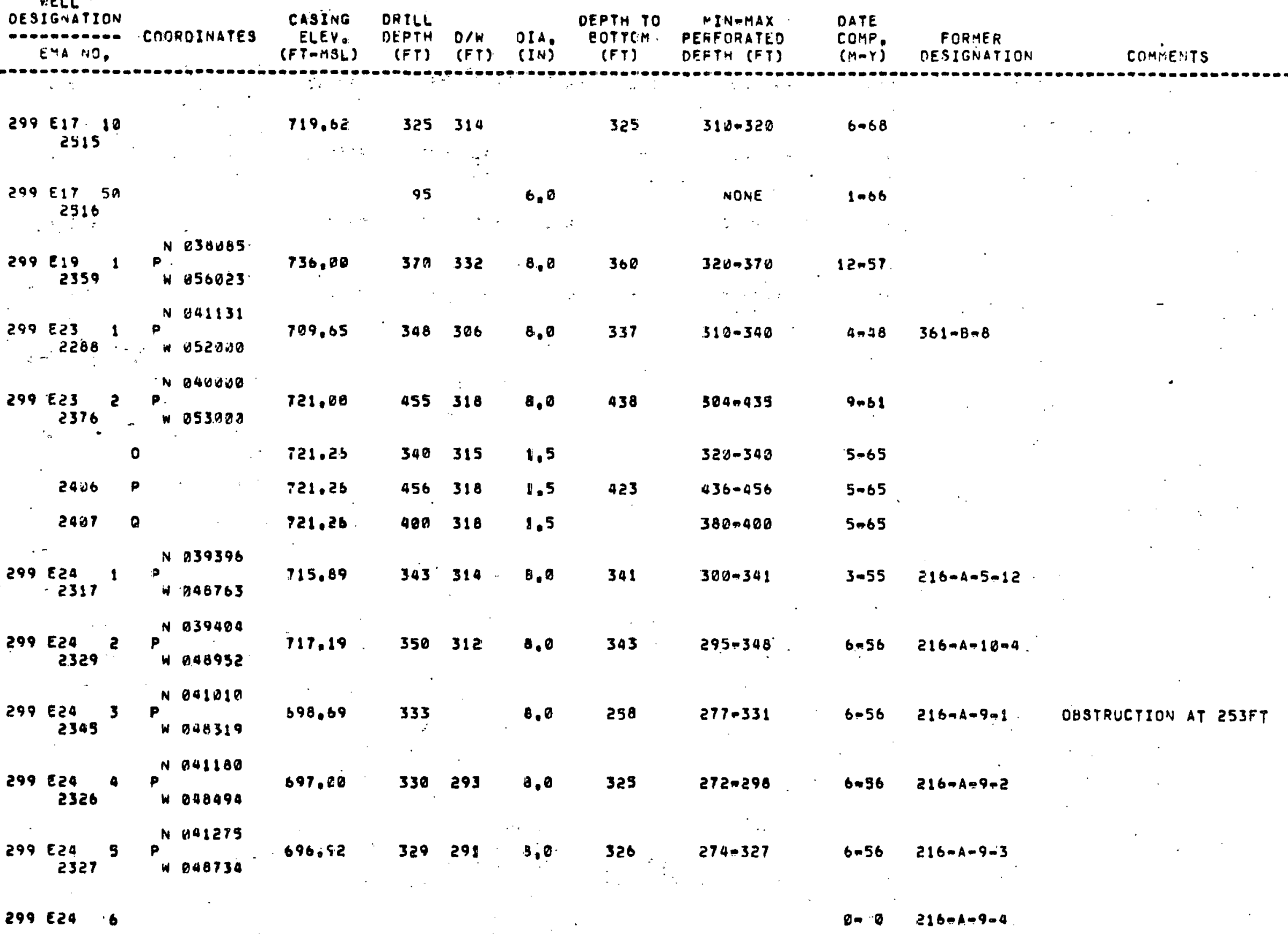




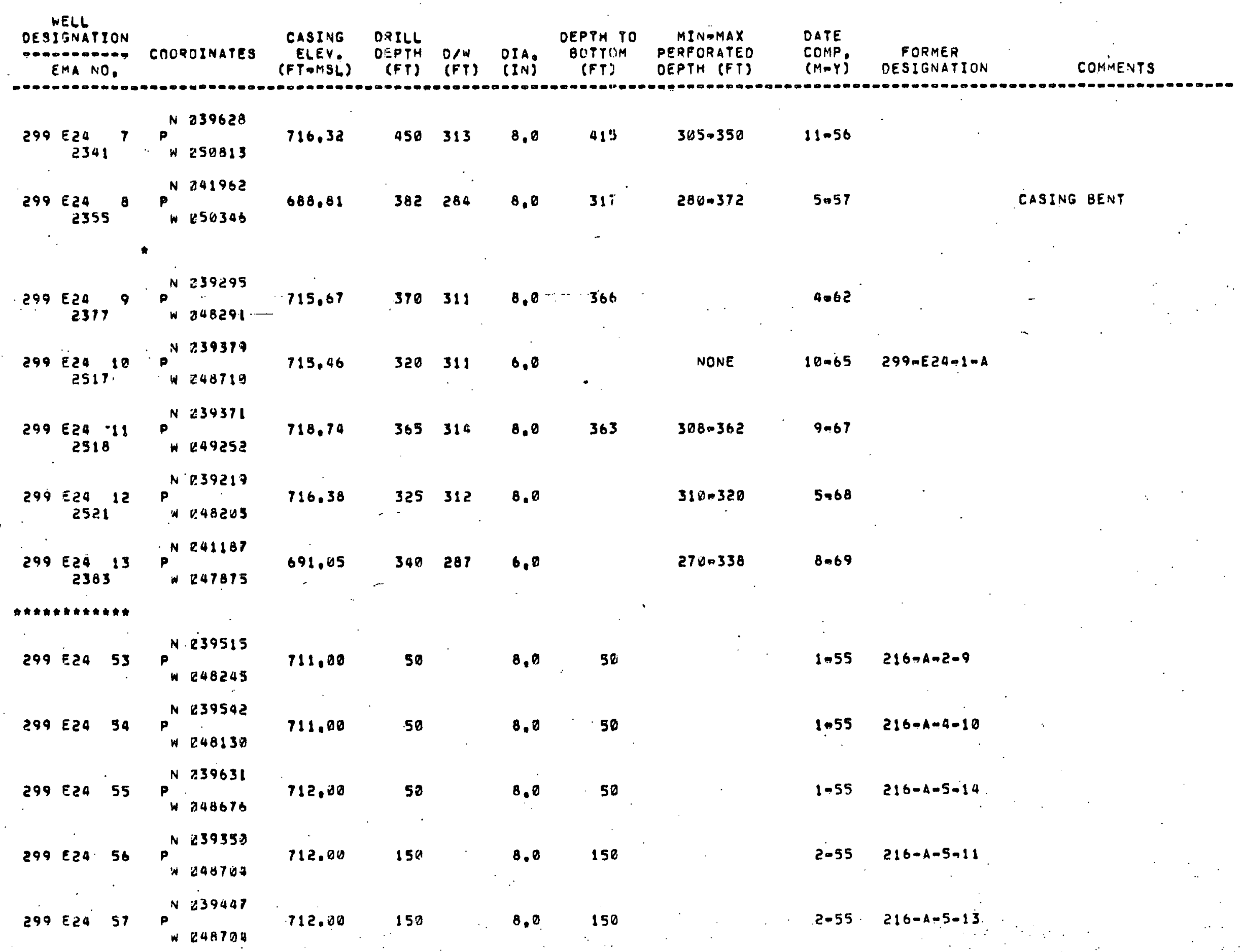




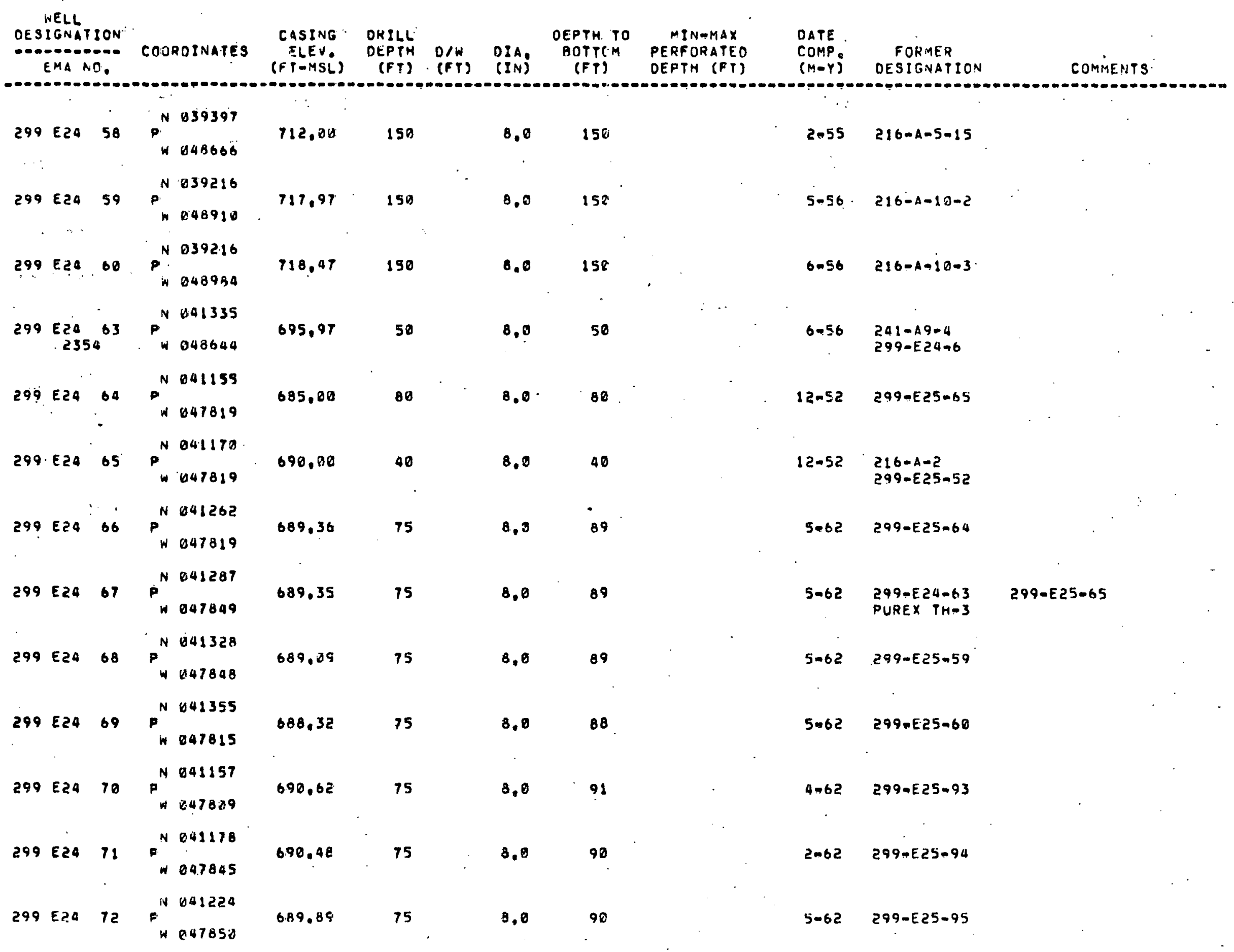




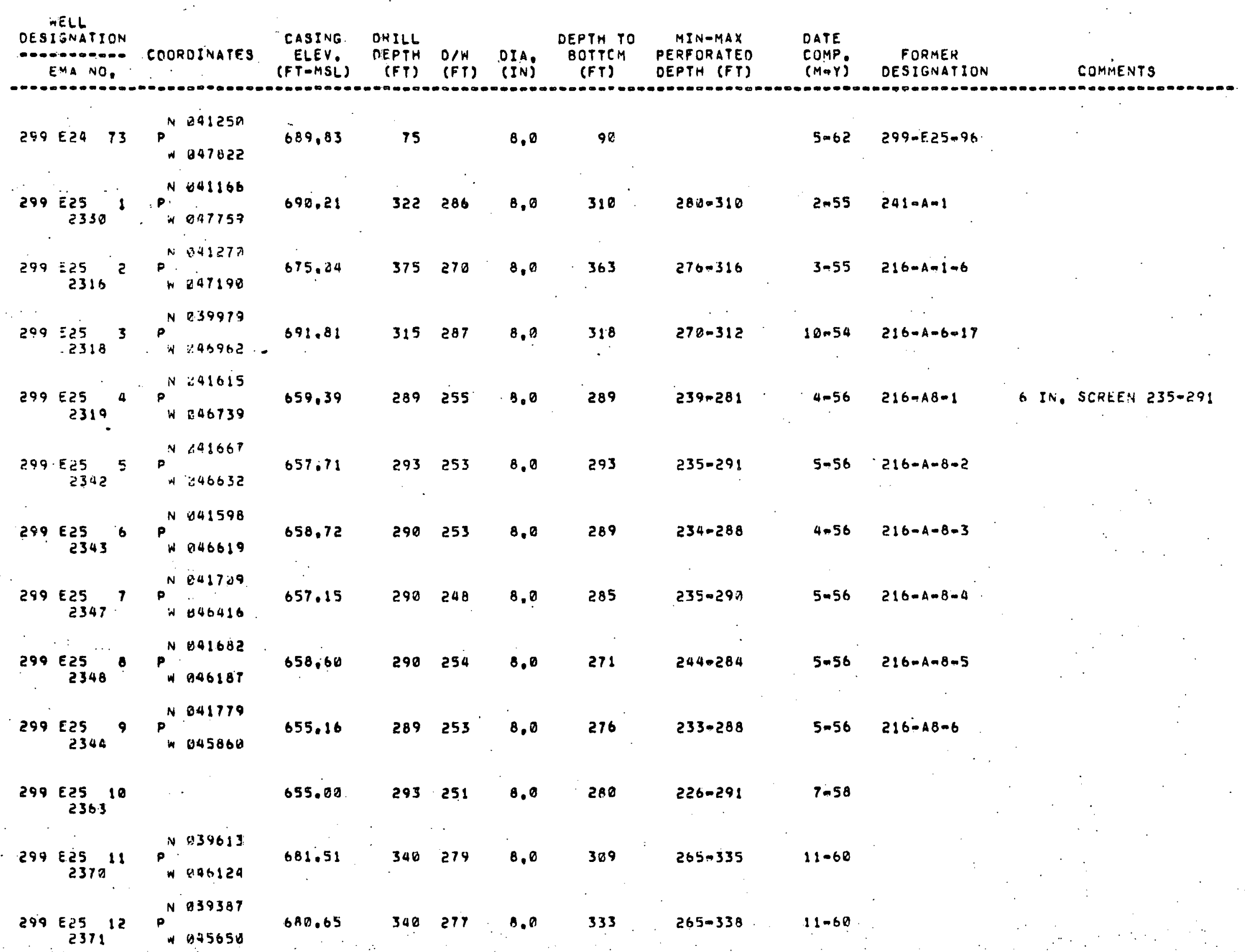




\begin{tabular}{|c|c|c|c|c|c|c|c|c|c|c|c|}
\hline DES & $\begin{array}{l}\text { VELL } \\
\text { IGVAT } \\
\text { EAAN }\end{array}$ & ION & COCROINATES & $\begin{array}{l}\text { CASING } \\
\text { ELEV. } \\
\text { (FT=MSL) }\end{array}$ & $\begin{array}{l}\text { DRILL } \\
\text { OEPTA } \\
\text { (FT) }\end{array}$ & $\begin{array}{l}0 / W \\
(F T)\end{array}$ & $\begin{array}{l}\text { OIA: } \\
\text { IIN) }\end{array}$ & $\begin{array}{c}\text { DEPTH TO } \\
\text { BOTTO:1 } \\
\text { (FT) }\end{array}$ & $\begin{array}{l}\text { MIN-MAX } \\
\text { PERFORATEO } \\
\text { DEPTH (FT) }\end{array}$ & $\begin{array}{l}\text { DATE } \\
\text { COMPP } \\
\text { (M-Y) }\end{array}$ & $\begin{array}{l}\text { FORMER } \\
\text { OESIGNATION }\end{array}$ \\
\hline 299 & $\begin{array}{l}\text { E25 } \\
252\end{array}$ & $3^{13}$ & $\begin{array}{l}\text { N04152? } \\
\text { N0407670 }\end{array}$ & 680.56 & 317 & 276 & 8.0 & 317 & $256-315$ & 10.63 & \\
\hline 299 & E25 & 14 & $:$ & & 208 & 254 & $\Leftrightarrow 0$ & & NÓNE & $\theta=0$ & . \\
\hline 299 & $\begin{array}{l}\text { E } 25 \\
252\end{array}$ & $2^{15}$ & $\begin{array}{l}N=041825 \\
P \quad 047658\end{array}$ & 689,67 & 340 & 284 & 6.0 & & $270-338$ & $7-69$ & $299-\varepsilon 25-1 \mathrm{~h}$ \\
\hline 299 & $\begin{array}{l}\text { E25 } \\
.230\end{array}$ & $4^{16}$ & $\begin{array}{l}P^{N} 241225 \\
* 047800\end{array}$ & 691.17 & 390 & 284 & 6,0 & $\therefore$ & $270-338$ & $7-69$ & $299-\varepsilon 24-15$ \\
\hline Ant & 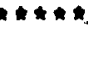 & *** & & . & & $\therefore$ & & & & & $\therefore$ \\
\hline 299 & E25 & 52 & . & & 40 & & 8,0 & . & & $12-52$ & $216-A-2$ \\
\hline Annt & $m+\infty x$ & n. & & & & & & & & & \\
\hline 299 & E25 & 58. & $\begin{array}{l}N 041205 \\
P^{N} 047169\end{array}$ & 674.00 & 150 & & 3.0 & 250 & & $3-55$ & $2: 6-A-7-18$ \\
\hline 299 & E25 & 55 & $\begin{array}{l}P^{N} 041223 \\
{ }_{N} \\
007651^{\circ}\end{array}$ & 688.33 & 151 & . & $B, 0$ & 151. & & $5-55$ & $241-A-3$ \\
\hline 299 & E25 & 56 & $\begin{array}{l}N 441273 . \\
N 247753\end{array}$ & $682.6 \bar{z}$ & 151 & & 1,0 & 151 & & $6-55$ & $241-A-2$ \\
\hline 299 & E25 & 57 & 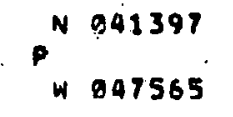 & 687.47 & 1.50 & & 8.0 & 150 & & $6-55$ & $291-A-4$ \\
\hline 299 & E25 & 58 & $\begin{array}{l}P^{N 041160} \\
W 047540\end{array}$ & 688.82 & 151 & & 8.0 & 151 & & 6.55 & $241-4 \div 5$ \\
\hline 299 & E25 & 61 & $\begin{array}{l}P_{N}^{N} 041345 \\
N 047774\end{array}$ & 080.11 & 151 & & 8,0 & 75 & . & 6.55 & $\because$ \\
\hline 299 & E25 & 62 & $\begin{array}{l}P_{W 041304}^{N 8} \\
W 07753\end{array}$ & 888.21 & 75 & & 8.0 & 75 & & $4-52$ & \\
\hline
\end{tabular}




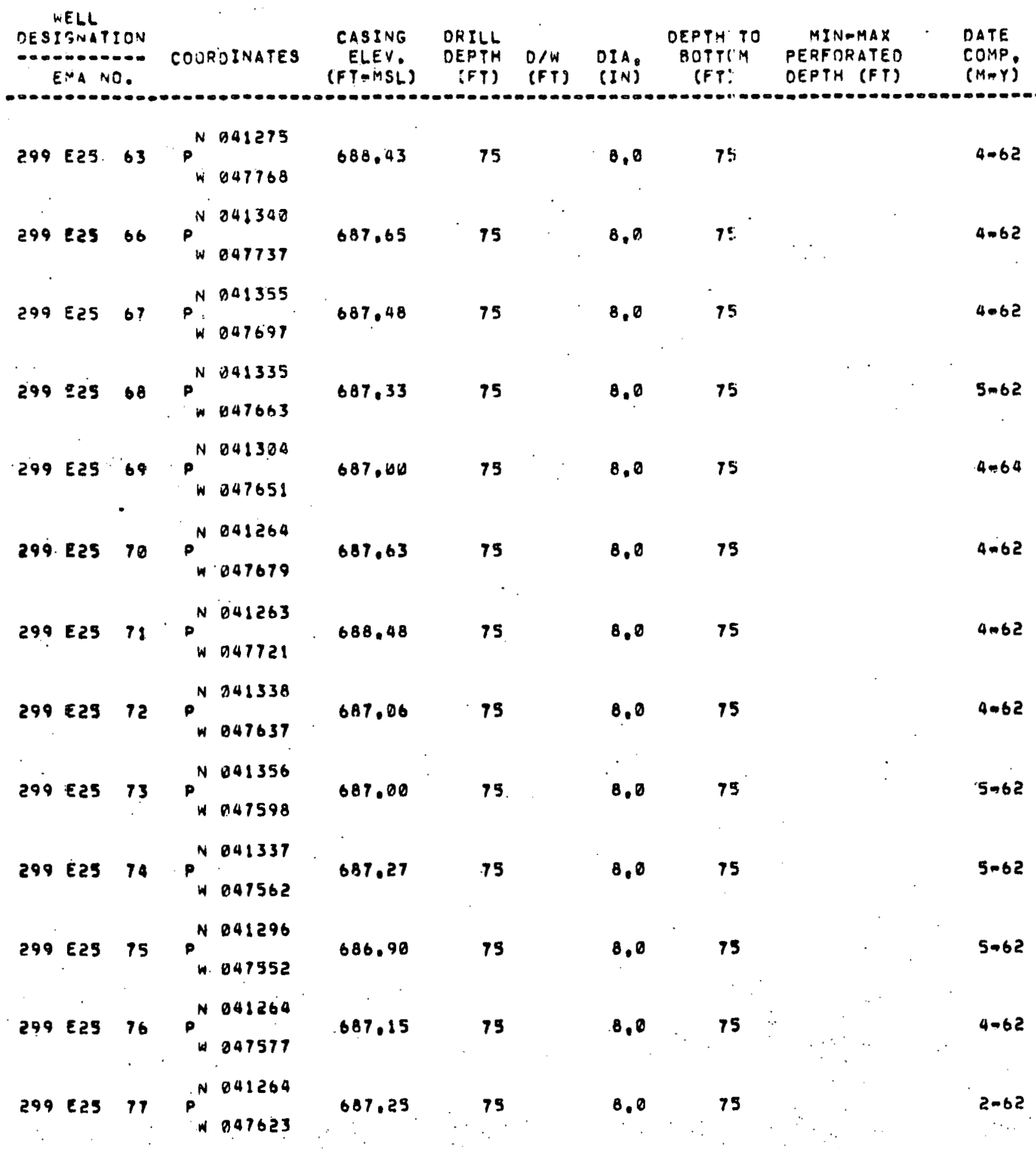


WELL

DESIGNATION
EMA NO. CODROINATES

CASING

DRILL

O/W OTA, DEPTH TO

MINGMAX
PERFORATED

DAYE

(FT-MSL)

(FT) (FT) (IN) (FT)

DEPTH (FP)

COMP.

$(M-Y)$

FORMER

DESIGNATION

COMMENTS

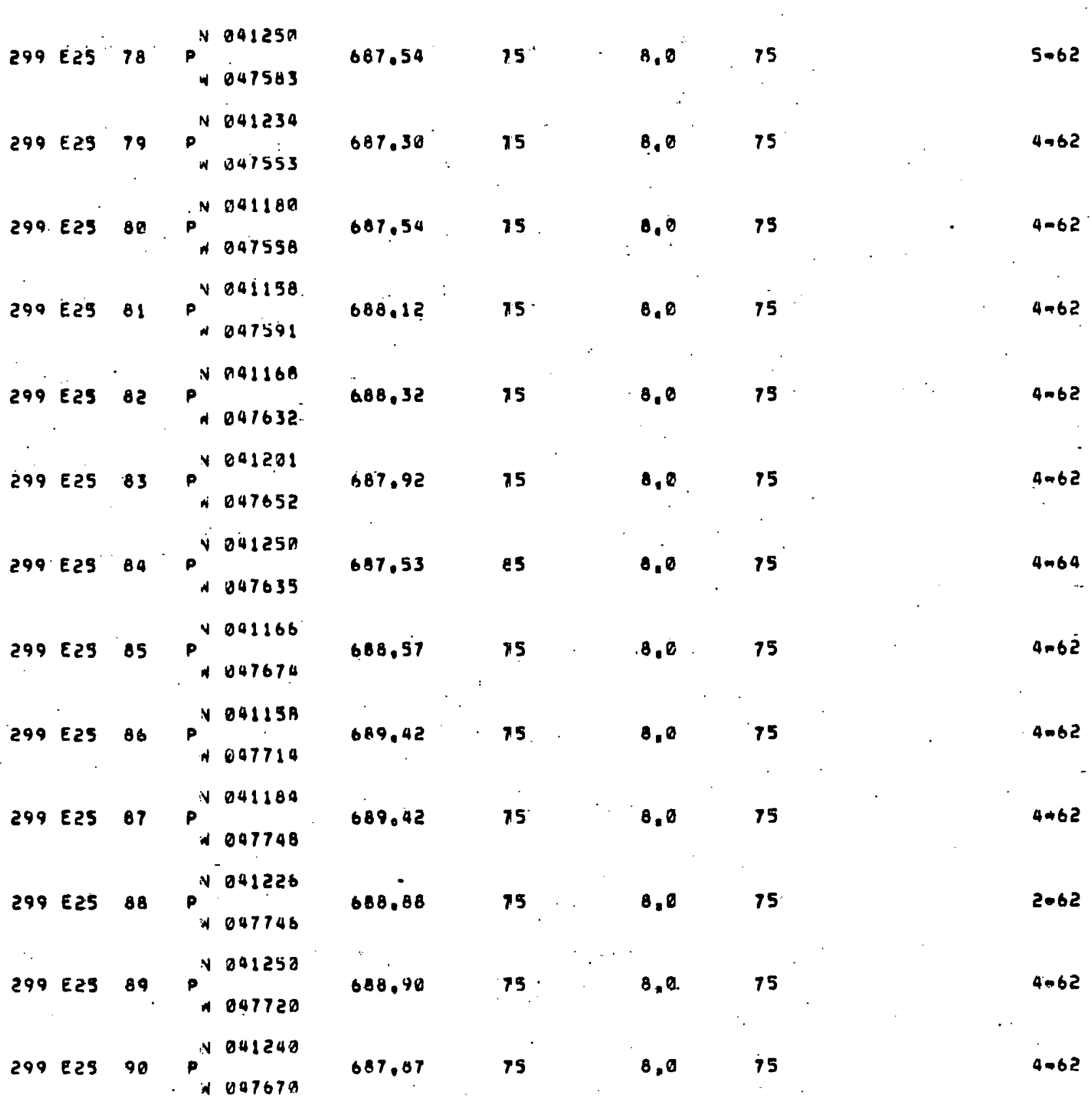


WELL

OESITNATION

COOROINATES

CASING OKILL

$\begin{array}{lll}\text { CASING } & \text { DEILL } \\ \text { ELEV. DEPTH O/W.. DIAD BOTTIOM PERTORATED }\end{array}$

DATE (FT-MSL)

(FT) (FT) (IN) (FT) DEPTH (FT)

COMP FURMER

(MAY) DESIGNATION

CUMMENTS

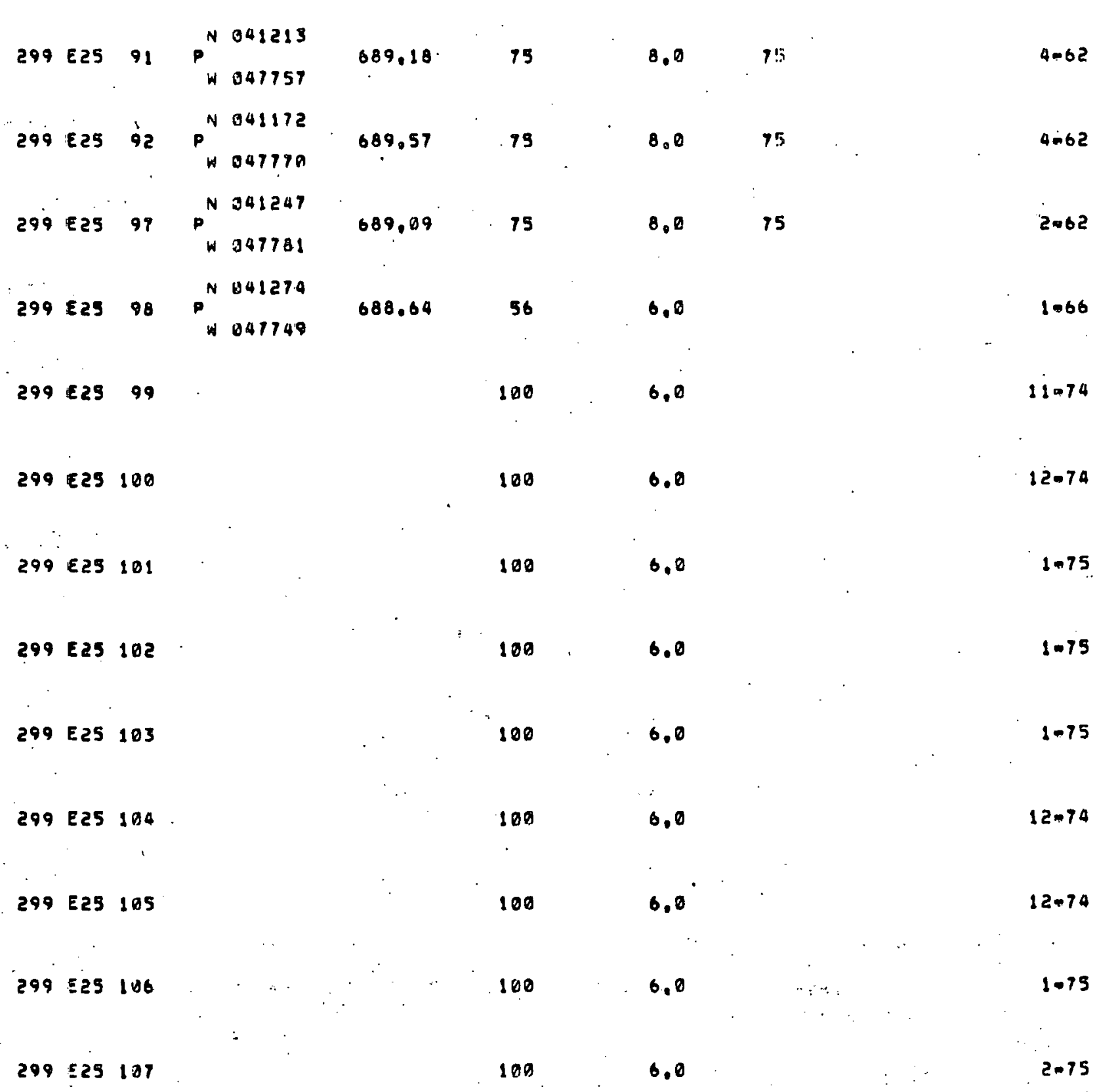


WELL

DESIGNATION

EMA NO.

COORDINATES

CAsINe:

ORILL

OEPTH TO
OIW DIA. BOTTO.4

ELEV.

DEPTH

MINEMAX
PERFORATED

DATE

(FT) (FI) (IN)

(FP)

DEPTH (FT)

COMP.

FORMER

DESIGNATION

COAMENTS

$299 E 25108$

100

6.0

$2-75$

299 EटS 109

100

6,0

2075

299 E23 110

190

$b, 0$

$2-75$

299 EaS 111

100

0.0

$2-75$

299. E2S 112

100

6,0

1075

100

0.0

$1=75$

299 E25 114

100

6,0

$12-74$

299 E2S 115

100

6.0

2.75

299 E25 116

129

6,0

1075

$299 \quad E 25117$

100

6.0

$1-75$

299 E25 118

100

6,0

12.74

$299 E 25119$

100

6.8

12074

$299 E 25 \quad 120$

100

6. 0

$2-75$ 
WELL

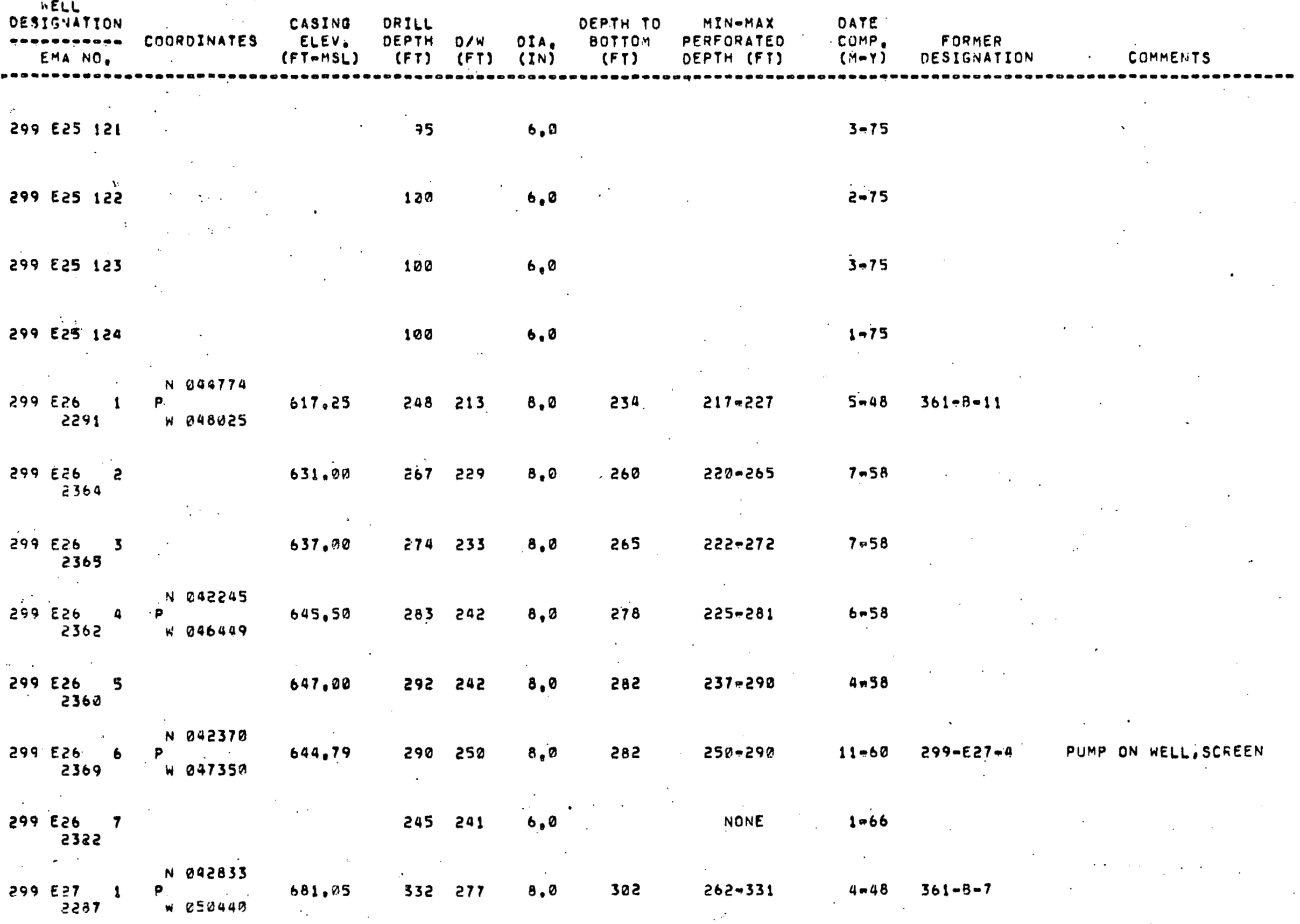




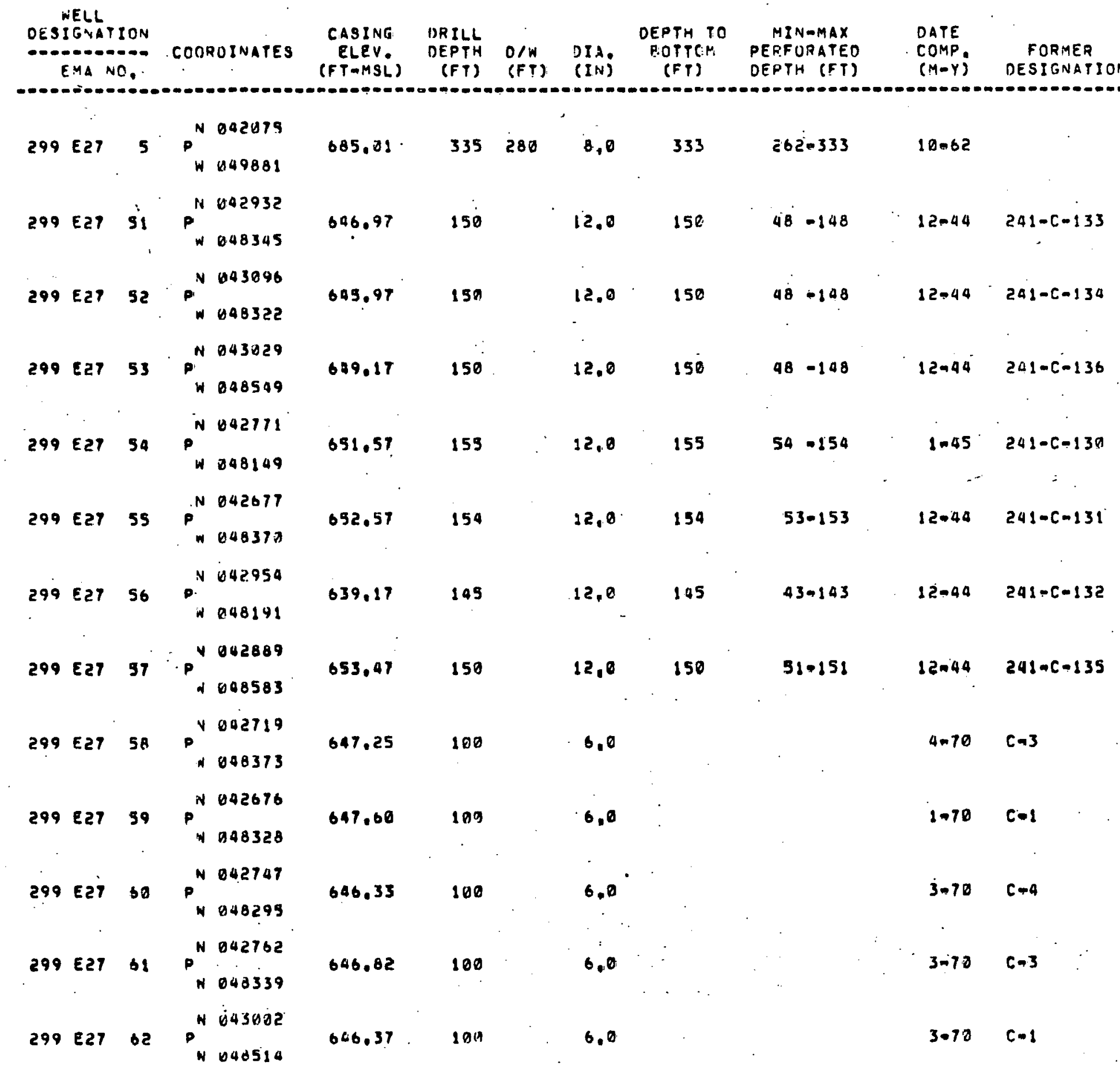


*ELL

OESIGNATION

EMA NO.

COOROINATES

CASING

DEPTH TO MINAMAX

DEPTH D/W OIA BOTYOY PERFORATEO

(FT) (PT) (IN) (FT) DEPTH (FT)

OATE

COMP. FORMER

(M-Y) DESIGNATION

COMMENTS

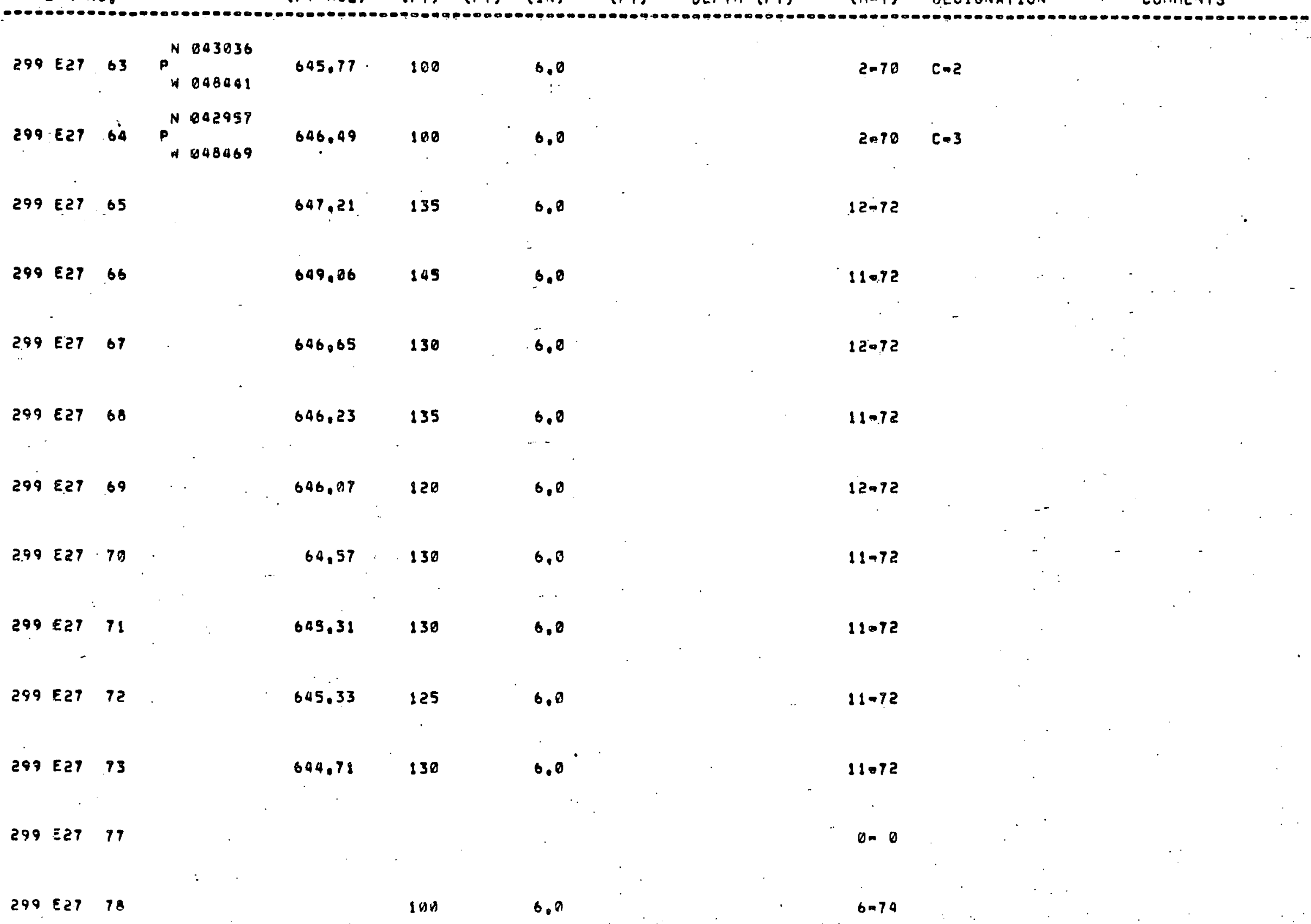


WELL

OESITNATION

oenganatgon

COOROINATES

CASING

DRILL

OEPTH TO

OEPTH O/W OIA, BOTTOM

MINAMAX

PERFORATED

DAPE (FT=MSL)

(FT) (FT: (IN) (FT)

DEPTH (FT)

COMP. FORMER

(M-Y) DESIGNATION

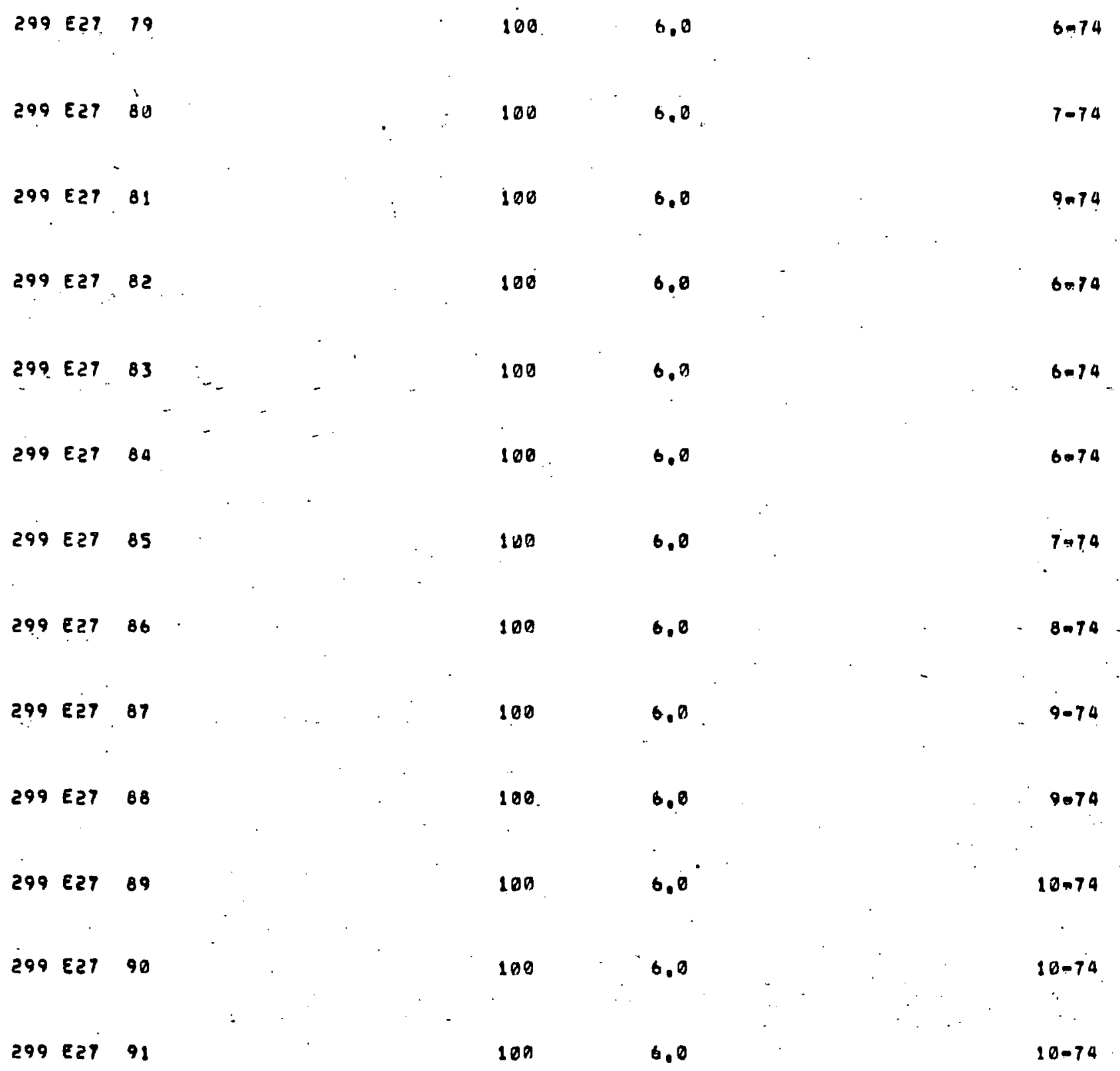


WELL

OESTGNATION

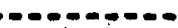

CASING URIL

DEPTH TO

ELEV. DEPTH O/W OIA BOTTOM

PERFORATED

DATE

COMP. FORMER

(M-Y) OESITNATION

COMME:UTS

EMA NO,
$0.04-M S W$ )

$299 \quad 627 \quad 92$

100

6.0

$9-74$

$299 \quad 627.93$

100

0,0

7.74

$299 E 27 \quad 90$

100

6.0

9.74

299 E27 99

100

6.8

$9=74$

290 ह27 96

109

6.0

7.74

$299 \quad E 27 \quad 97$

100

6.0

6.74

$299 \quad 527 \quad 98$

100

6.0

$9-74$

$299 E 27 \quad 99$

100

6.0

$7=74$

$299 \leq 27 \quad 100$

100

6.0

$7=74$

$299527 \quad 101$

100

6.0

9.74

$299 \quad 27102$

100

$6.0^{\circ}$

$9=74$

$299 \quad E 27 \quad 103$

100

6.0

$9 \cdot 74$

299 E27 104

109

6.0

$4-75$ 
WELL

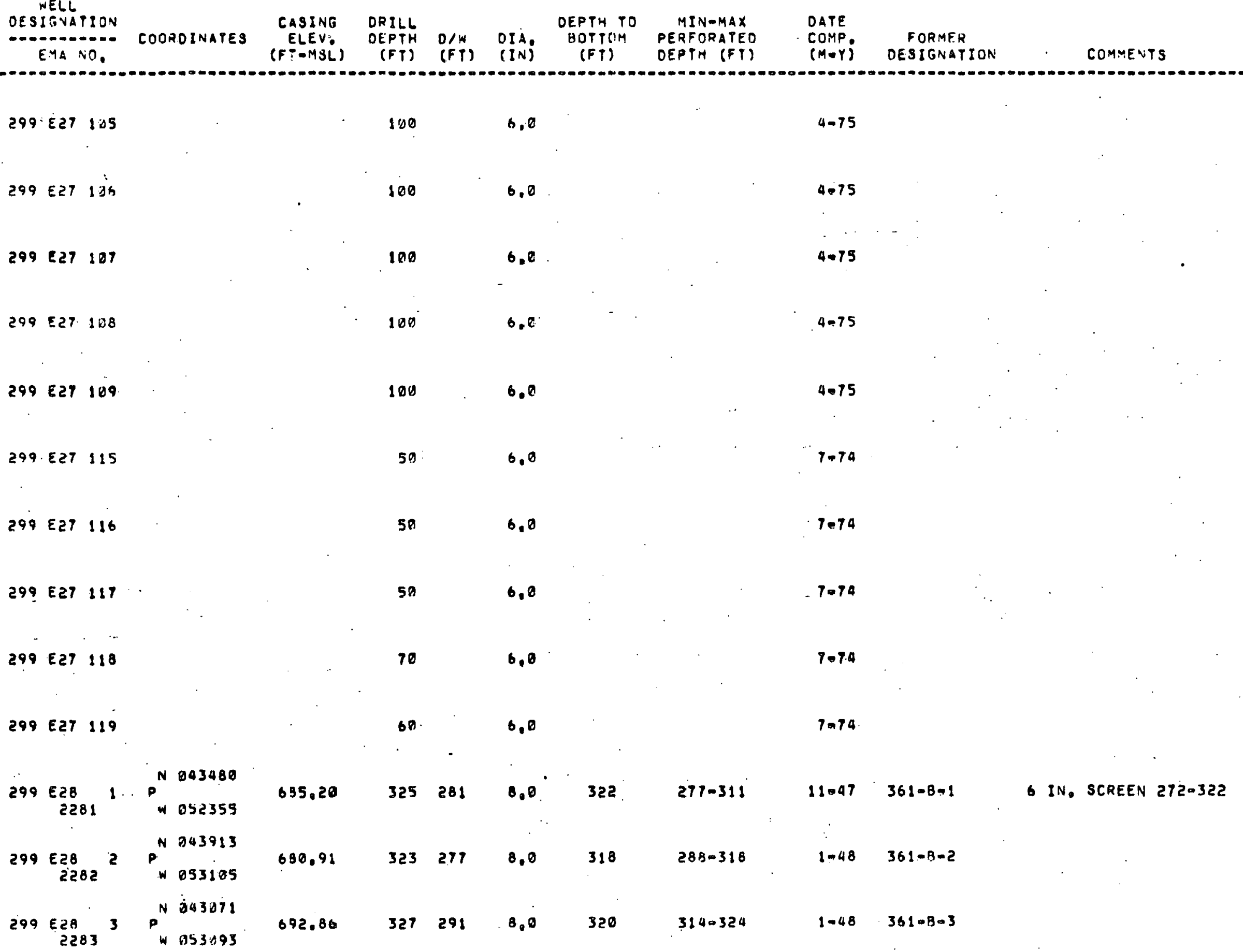




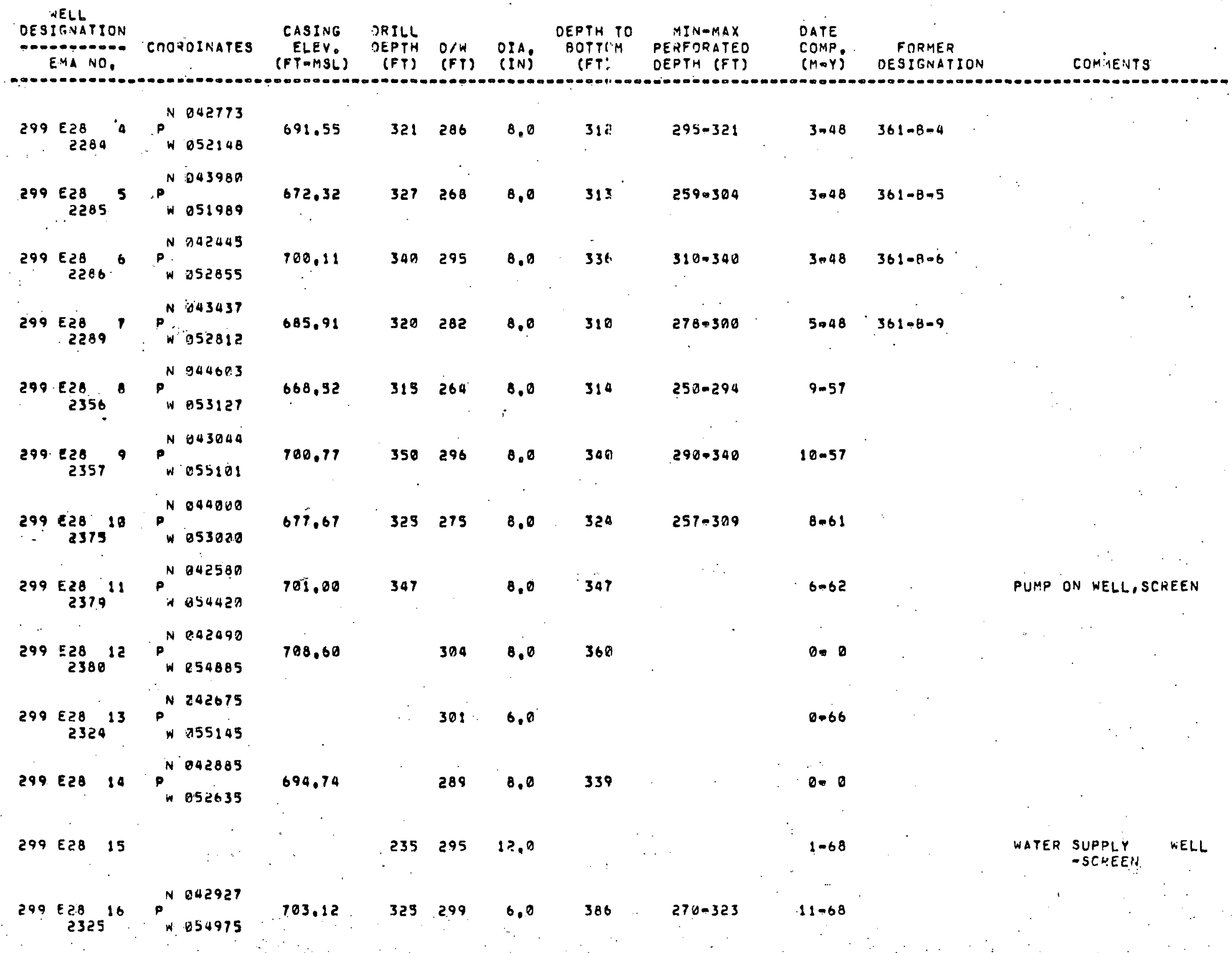




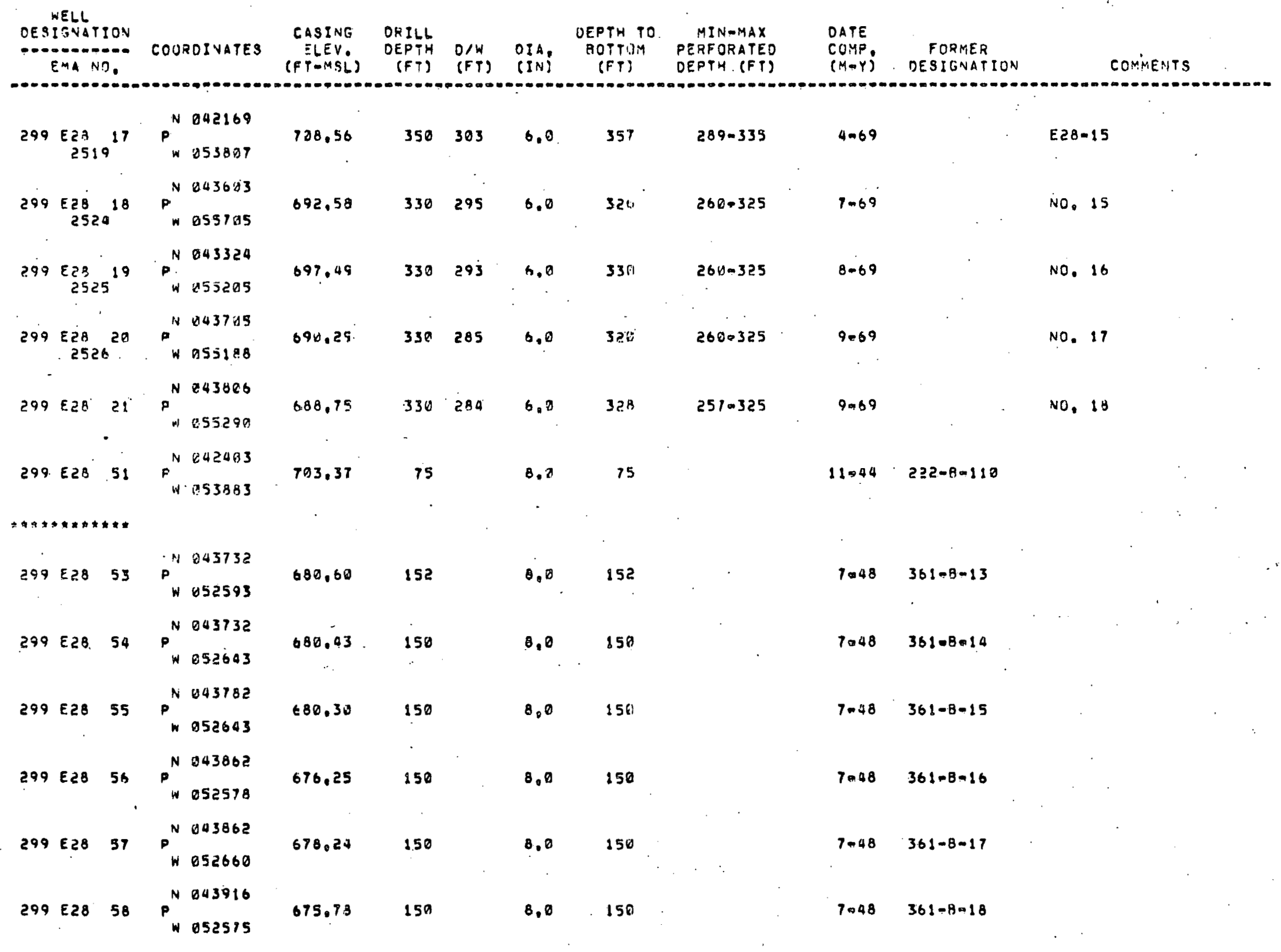




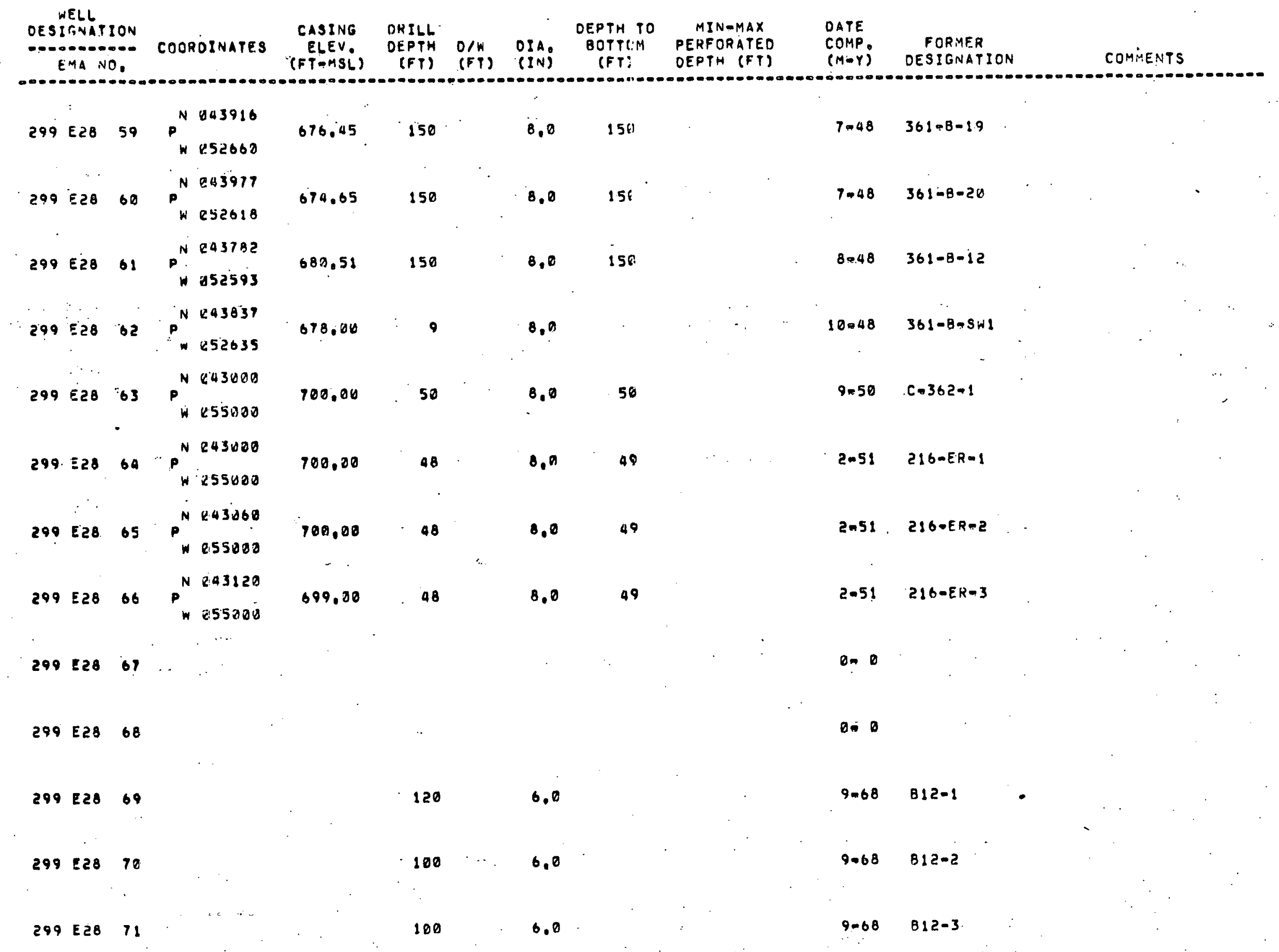




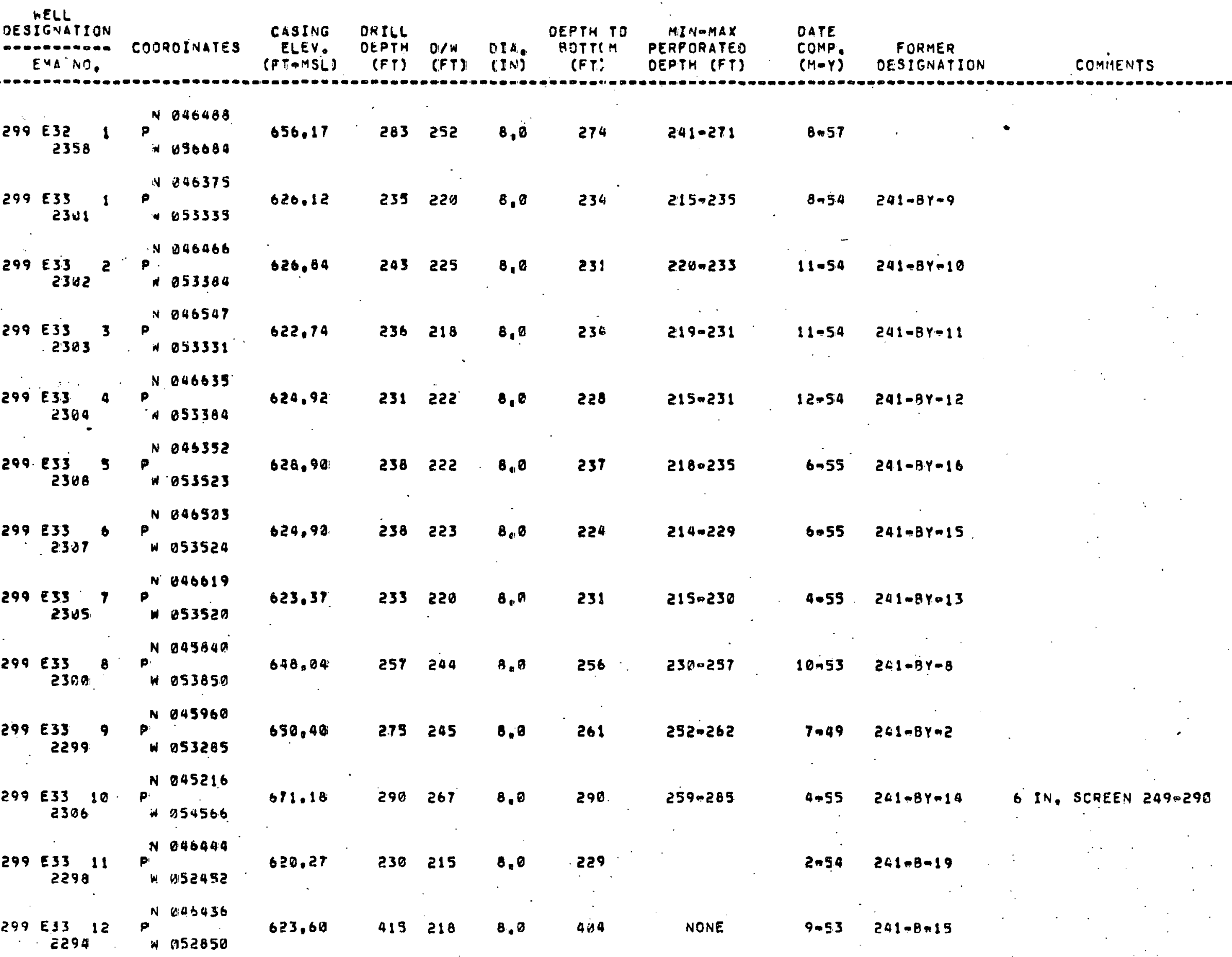




\begin{tabular}{|c|c|c|c|c|c|c|c|c|c|c|}
\hline OES & $\begin{array}{l}\text { WLL } \\
\text { ISNAYION } \\
\text { EMA NOE. }\end{array}$ & COORCINATES & $\begin{array}{l}\text { CASING } \\
\text { ELEV. } \\
\text { (FTAMSL) }\end{array}$ & $\begin{array}{l}\text { DRILL } \\
\text { DEPTH } \\
\text { (FT) }\end{array}$ & $\begin{array}{l}0 / W \\
(F T)\end{array}$ & OIA: & $\begin{array}{l}\text { DEPTH TO } \\
\text { BOTTCM } \\
\text { (FT) }\end{array}$ & $\begin{array}{l}\text { MINAMAX } \\
\text { PERFDRATEO } \\
\text { OEPTH (FT) }\end{array}$ & $\begin{array}{l}\text { DATE } \\
\text { COMP } \\
\text { (M-Y) }\end{array}$ & $\begin{array}{l}\text { FORMER } \\
\text { OESIGNATI }\end{array}$ \\
\hline 299 & $\underbrace{E 33}_{2296} 13$ & $\begin{array}{l}P^{N} 246278 \\
\quad 053093\end{array}$ & 625.53 & 235 & 220 & 8.0 & 234 & 210.235 & 18.53 & $241-B-17$ \\
\hline 299 & $\begin{array}{l}E 33 \\
2297^{24}\end{array}$ & $\begin{array}{l}P^{N} 046223 \\
\text { W }\end{array}$ & 622.12 & 230 & 218 & 8,0 & 230 & $212=227$ & $12-53$ & $241-8=18$ \\
\hline 299 & $\underbrace{833}_{2293} 15$ & $\begin{array}{l}N 046806 \\
P^{N} 052751\end{array}$ & 627.00 & 251 & 218 & 0,0 & 251 & $222-237$ & 2.53 & $241-8-11$ \\
\hline 299 & $\begin{array}{l}E 33 \\
2292^{16}\end{array}$ & $\begin{array}{l}P^{N} 04588 T \\
W 552815\end{array}$ & 635.51 & 258 & 229 & 8,0 & 298 & $231-246$ & 2053 & $241-8-5$ \\
\hline 299 & $\begin{array}{l}E 33 \\
2295^{17}\end{array}$ & $\begin{array}{l}P^{N} 645894 \\
\text { W } \\
P^{2} 2528\end{array}$ & 631.66 & 244 & $226^{\circ}$ & 0.0 & 244 & $220-244$ & 10.53 & $241-B=16$ \\
\hline 299 & ${ }_{2309}^{13} 18$ & $\begin{array}{l}P^{N} 025624 \\
N 252825\end{array}$ & 643.89 & 278 & 239 & 8,0 & 263 & $240 m 260$ & $2-50$ & $224-8=4$ \\
\hline 299 & E33 2331 & $\begin{array}{l}P^{N} 045744 \\
W 052629\end{array}$ & 639.00 & 252 & 238 & 0,0 & 243 & $217-248$ & 6.56 & $603-8 \div 1$ \\
\hline 299 & $\begin{array}{c}E 33 \\
2332^{29}\end{array}$ & $\begin{array}{l}P^{N 045664} \\
W 052620\end{array}$ & 640.87 & 254 & 238 & 8,0 & 250 & $225-25 !$ & 7.56 & $603-B-2$ \\
\hline 299 & $\underbrace{E 33}_{2353}$ & $\begin{array}{l}P^{N} 045321 \\
W 053856\end{array}$ & 663.43 & 282 & 258 & 8,0 & 230 & $235-275$ & 4.57 & . \\
\hline 299 & E33 22 & & 025.79 & 232 & 222 & 6.0 & & NONE & 8.65 & E33-2A \\
\hline 299 & ${ }_{2323}^{233}$ & & 625.34 & 230 & 220 & 6.0 & & NONE & 9.65 & $E 33-4 A$ \\
\hline 299 & $\begin{array}{l}E 33 \\
2520\end{array}$ & . & 638.03 & 256 & 233 & 8,0 & & $219-241$ & $5-67$ & \\
\hline 299 & $\begin{array}{c}E \geq 3 \\
2381\end{array}$ & 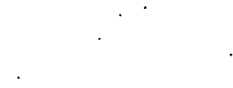 & 631.82 & 249 & 227 & 6.0 & & $199-233$ & 2.69 & \\
\hline
\end{tabular}


NELL

OESIGNATION

CoORginates EMA NO.

CASING DRELL

DEPTH D/W OER. DEPTH BOTYG MINAPAX BOTTCIM PERFORATED

\begin{tabular}{|c|c|c|c|c|c|c|c|c|c|c|c|c|}
\hline 299 & $\begin{array}{l}E 33 \\
238\end{array}$ & $2^{26}$ & & $\cdot$ & 632.82 & 248 & 229 & 0.0 & & & $3=69$ & \\
\hline 299 & $\begin{array}{l}E 33 \\
252\end{array}$ & $7^{27}$ & & & . & & 251 & 9,0 & & & $\theta=0$ & \\
\hline 299 & E33 & 51 & $\dot{P}^{N}$ & $\begin{array}{l}045374 \\
653488\end{array}$ & 652.57 & 150 & & 12,0 & 149 & $49-149$. & $11-44$ & $241-8-130$ \\
\hline 299 & $\{33$ & 52 & $P^{N}$ & $\begin{array}{l}845176 . \\
\text { es255? }\end{array}$ & $659.0 ?$ & 153 & & 12,0 & 154 & $52-152$ & $11=44$ & $271-8-131$ \\
\hline 299 & E33 & 33 & $p^{N}$ & $\begin{array}{l}845508 \\
052615\end{array}$ & 646.41 & 152 & & 12.0 & 953 & $50-149^{\circ}$ & $12-44$ & $241-8-132$ \\
\hline 299 & $E 33$ & $5 a$ & $P^{N}$ & $\begin{array}{l}045375 \\
452110\end{array}$ & 652.79 & 179 & & 12.0 & 170 & $50-150$ & $12=44$ & $241-8-133$ \\
\hline 299 & E33 & 55 & $P^{N}$ & $\begin{array}{l}045580 \\
052815\end{array}$ & 651.72 & 159 & & 12.0 & $159^{\circ}$ & $50-150$ & $12 m 44$ & $241-8-134$ \\
\hline 299 & $\{33$ & 56 & $P_{N}^{N}$ & $\begin{array}{l}045177 \\
052853\end{array}$ & 656,20 & 150 & & 12,8 & 251 & $48-148$ & $12=44$ & $241 \div 8-135$ \\
\hline 299 & E33 & 97 & $p^{N}$ & $\begin{array}{l}049320 \\
052928\end{array}$ & 659.86 & 150 & & 92,0 & 150 & $49-149$ & $12-44$ & $241-B-136$ \\
\hline 299 & E33 & 58 & $P_{W}^{N}$ & $\begin{array}{l}045633 \\
052761\end{array}$ & 642,82 & 1.53 & & 0.0 & 153 & & $5-47$ & $224=8-1$ \\
\hline 299 & $\begin{array}{r}\text { E33 } \\
.\end{array}$ & 59 & $P_{W}^{N}$ & $\begin{array}{l}045633 \\
852794\end{array}$ & 643,83 & 253 & & 2.0 & 153 & & $3=47$ & $224-6-2$ \\
\hline 297 & E33 & 60 & $\Rightarrow_{W}^{N}$ & $\begin{array}{l}045606 \\
052777\end{array}$ & 643,67 & $153^{\circ}$ & & 8.2 & 153 & & $5-47$ & $224-8-3$ \\
\hline 299 & E33 & 61 & $P_{W}^{N}$ & $\begin{array}{l}045537 \\
053145\end{array}$ & 653.23 & $990^{\circ}$ & & 8,0 & 150 & $40-800$ & $21=47$ & $248+8 x-130$ \\
\hline
\end{tabular}


WELL

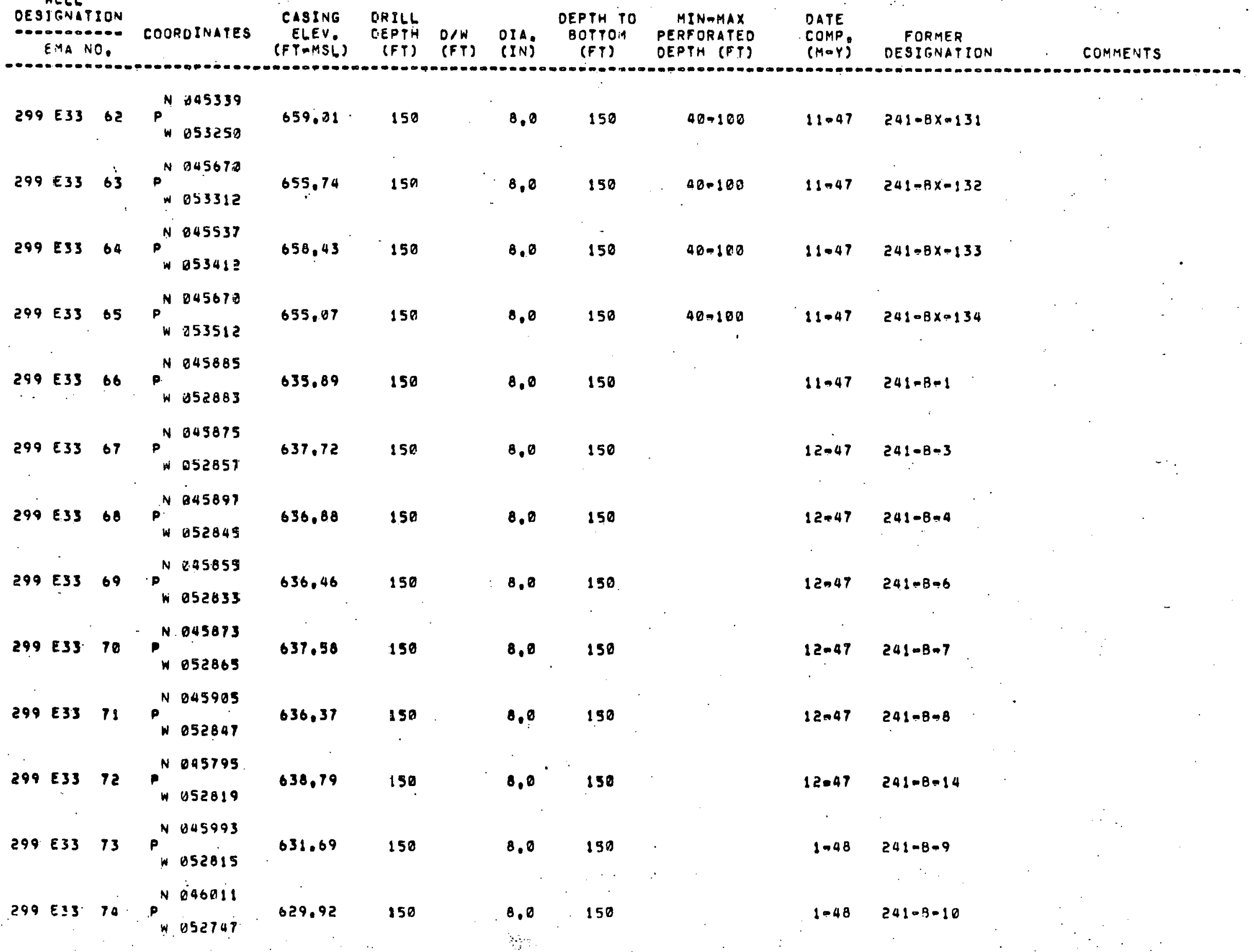




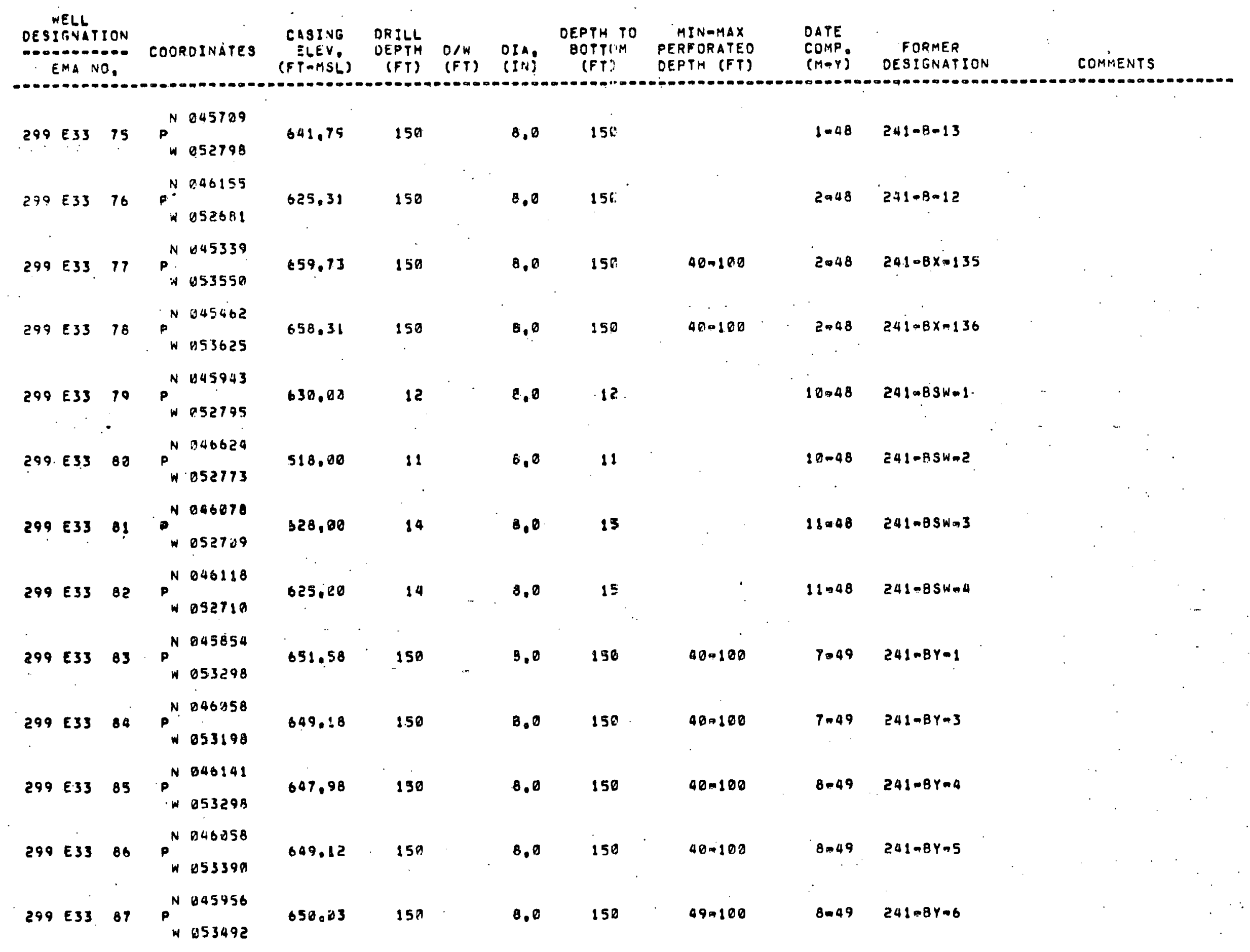


ELL

DESIGVATION

EMA NO.

COOROINAPES

CASING

ORILL

FimSi)

DEPTH TO MIN-MAX
DEPTH O/W DIA BOTTCM PERFORATED

BOTTCM PERFORATED

DATE

$(M \rightarrow Y)$

FORMER

OESIGNATION

COMMERTS

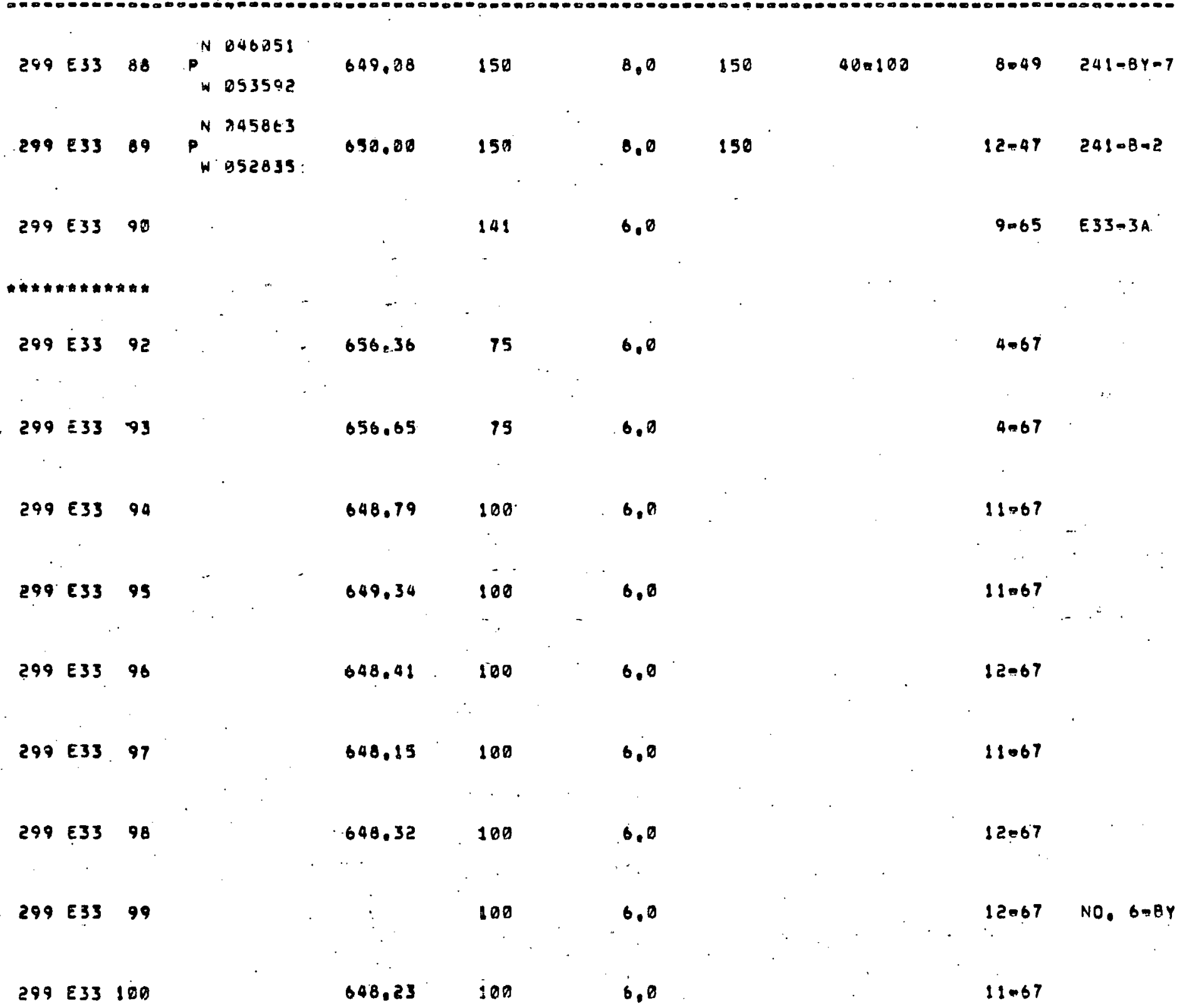




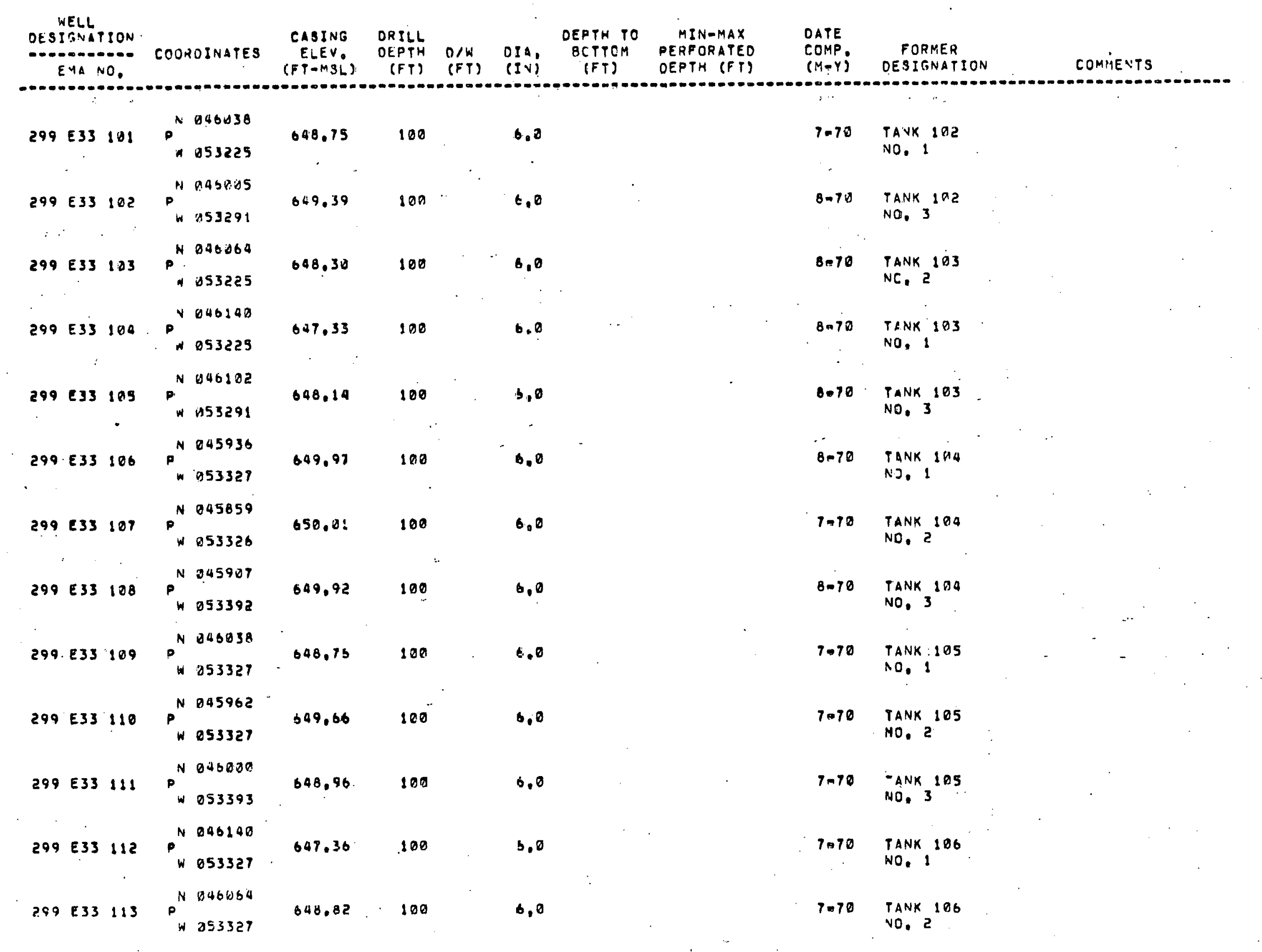




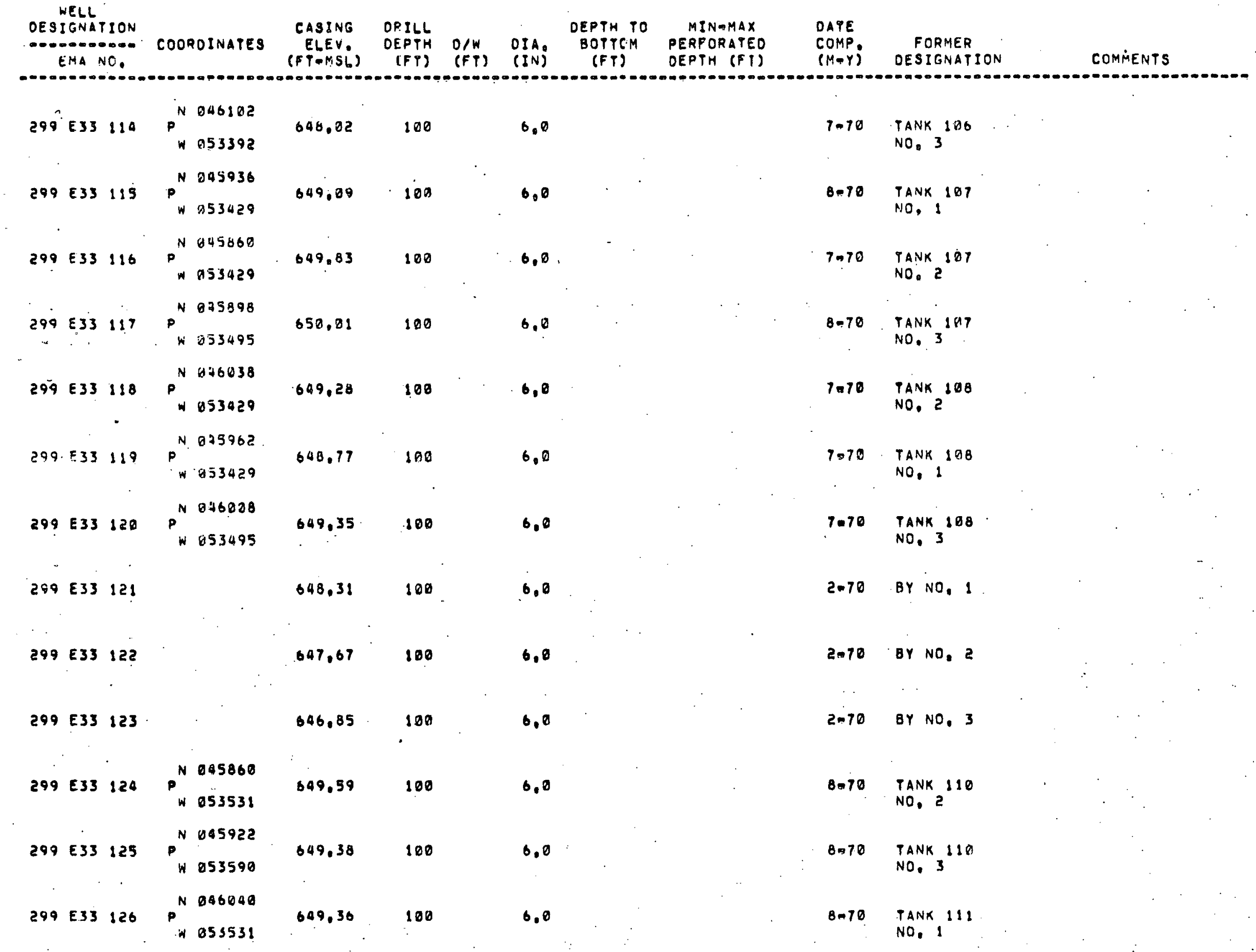




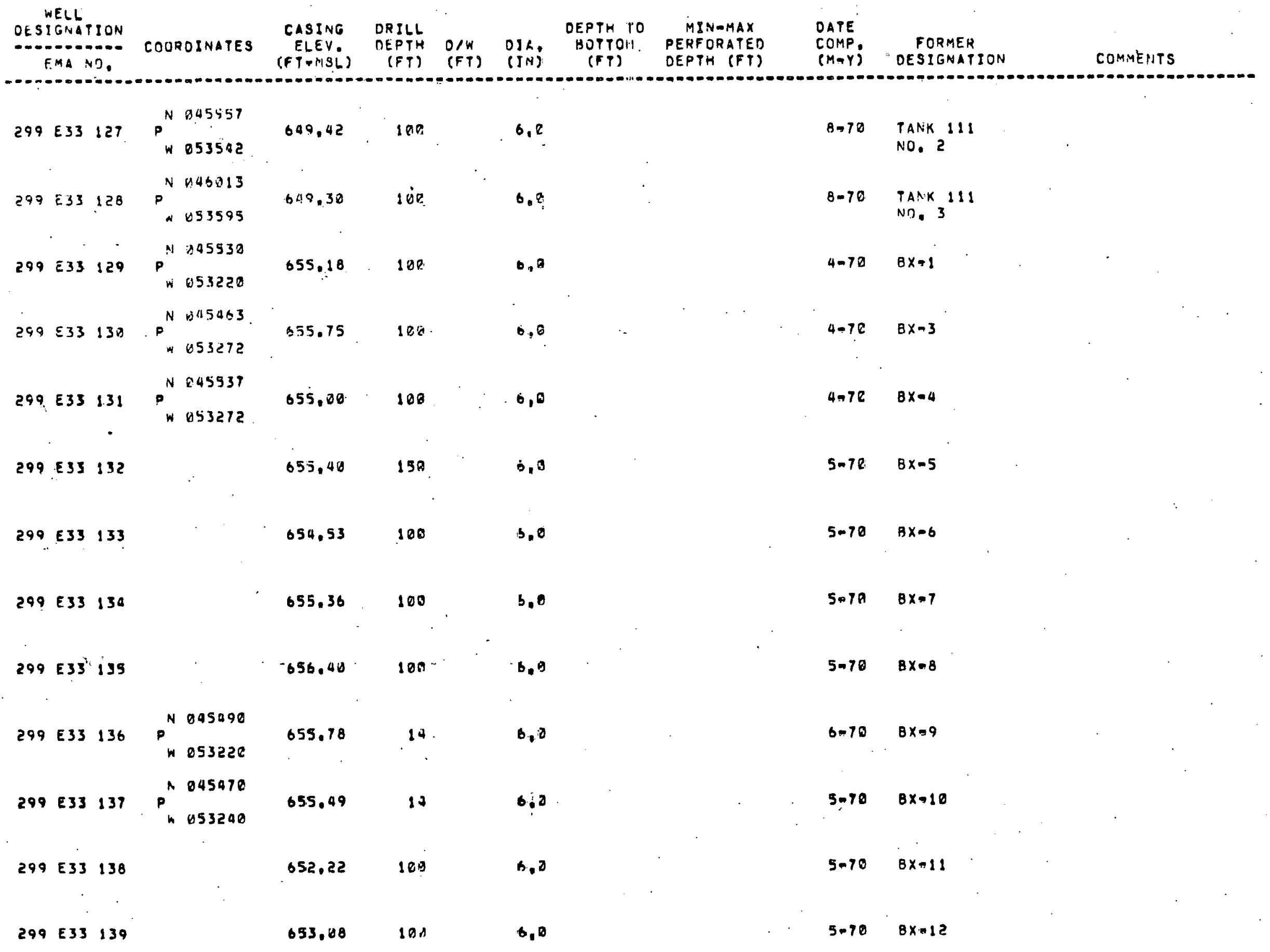


WELL

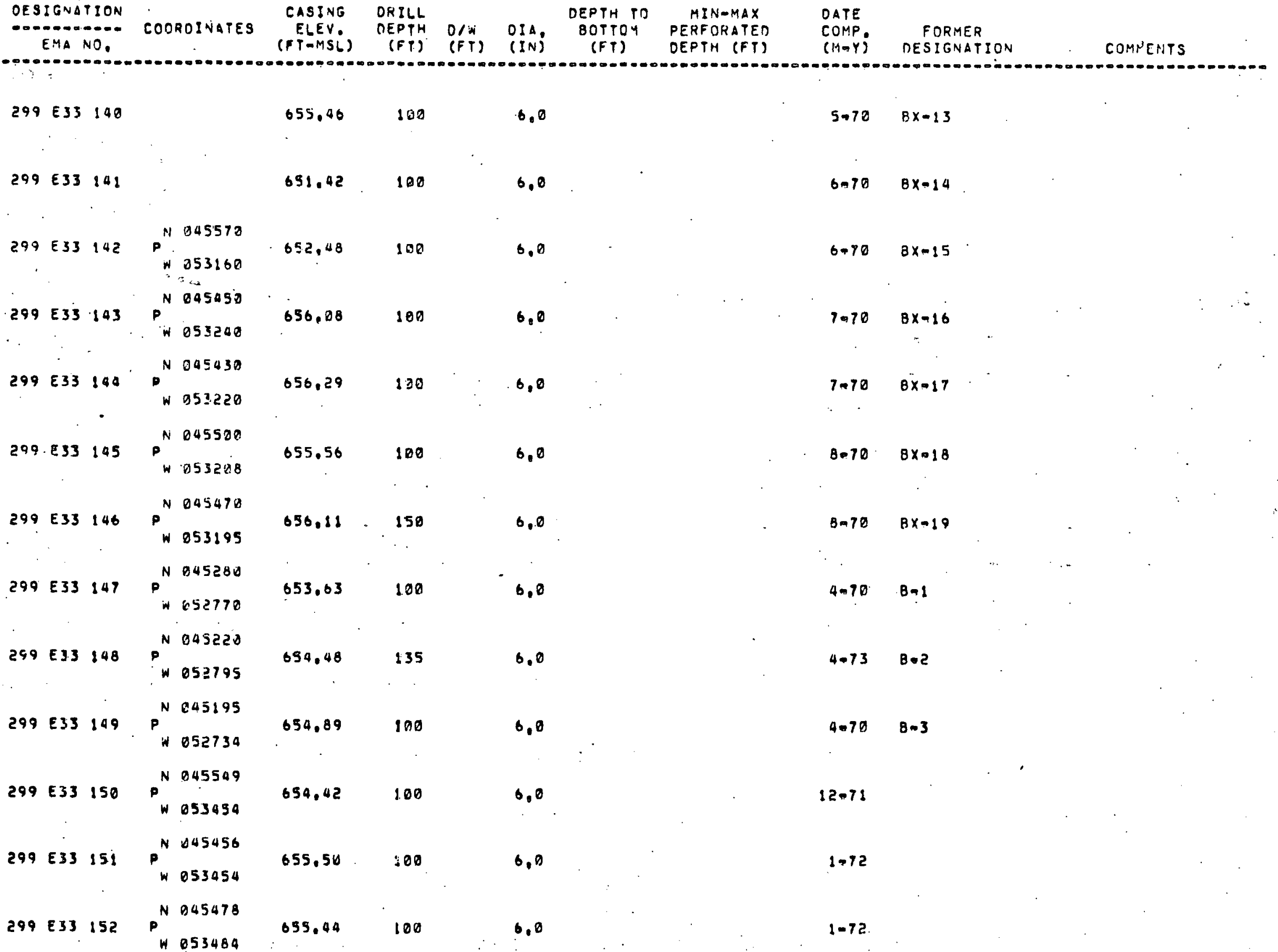




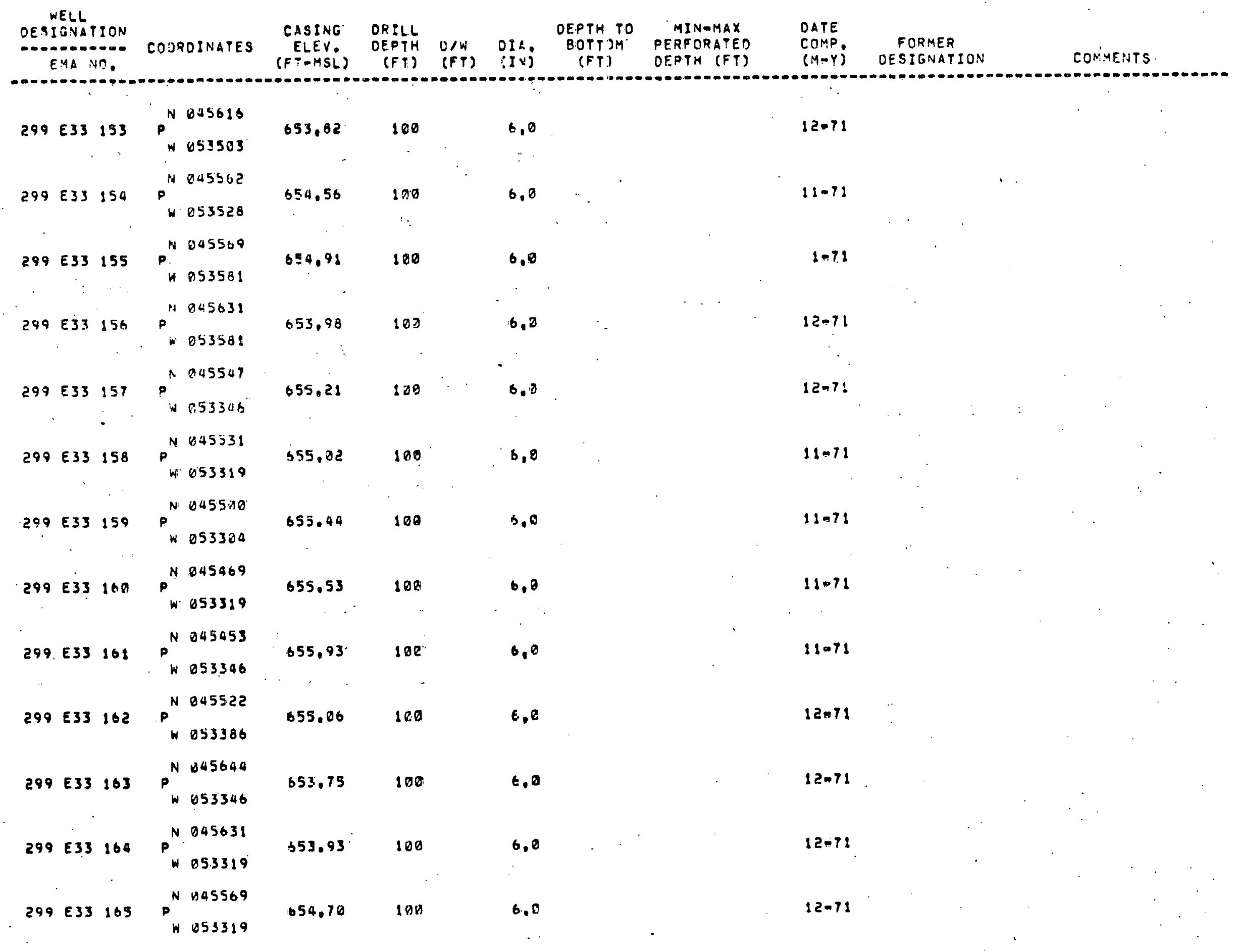


WELL

OESIGNITION

EMA 40

CASING DHILL OEPTH TO MINAMAX

DATE

COMP FORMER

(MAY) OESIGNATION

COMIIENTS

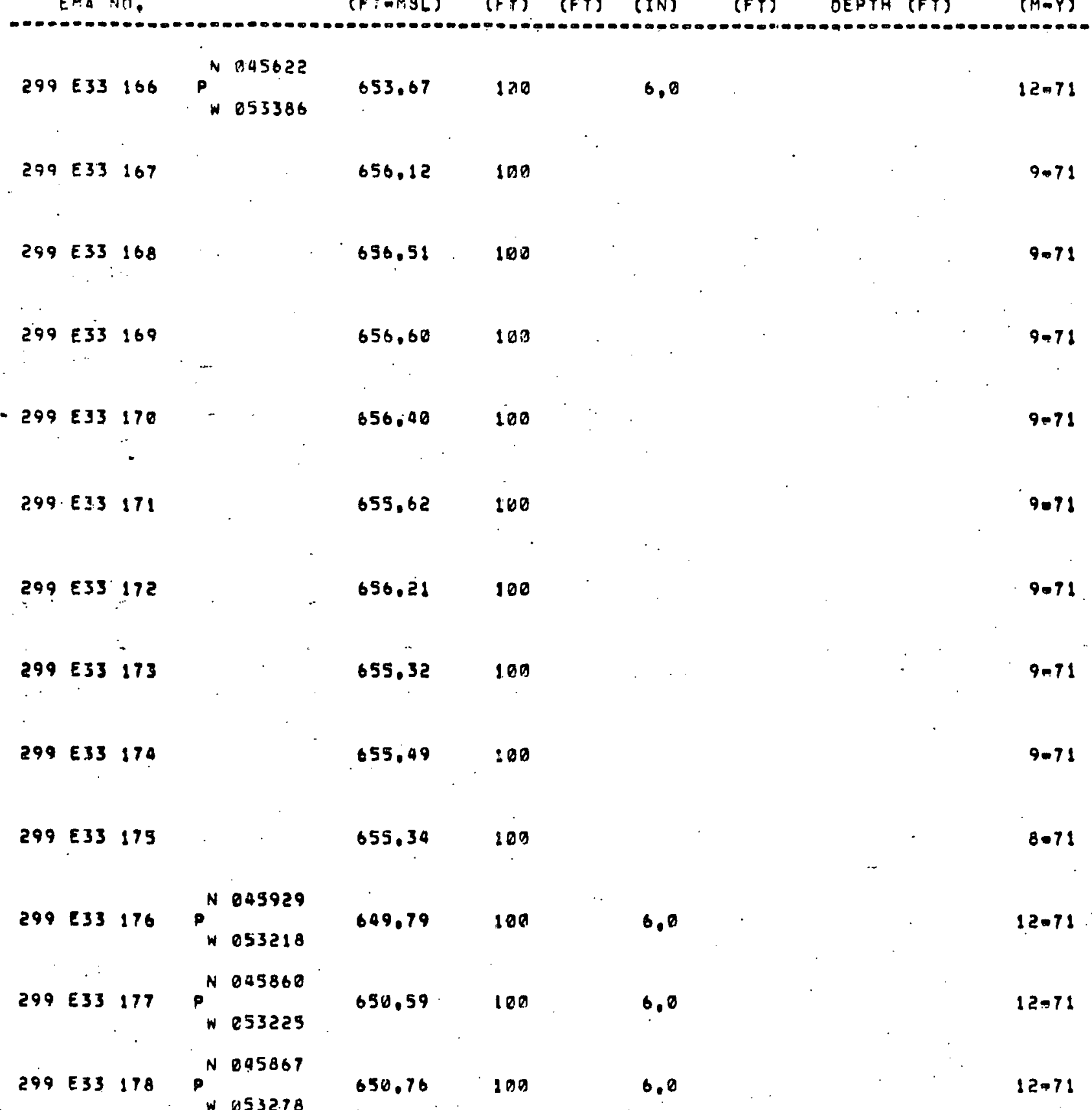


WELL

DESIGNATION COCROINATES

EMA NO.

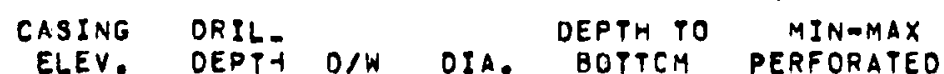

PERFORATED

DATE

ET-MSL) (FT) (FT) (IN) (FT) DEPTH (FT)

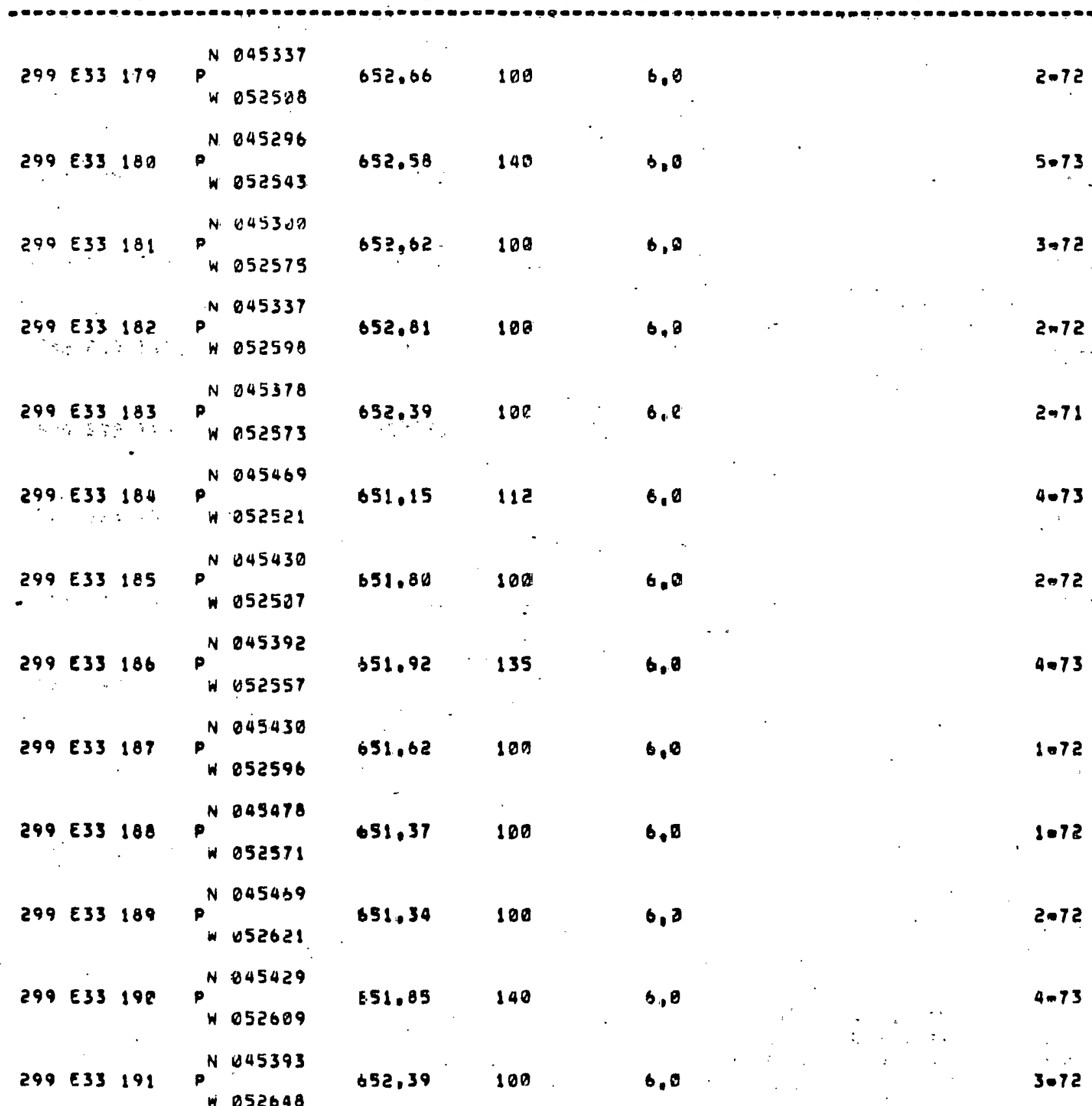




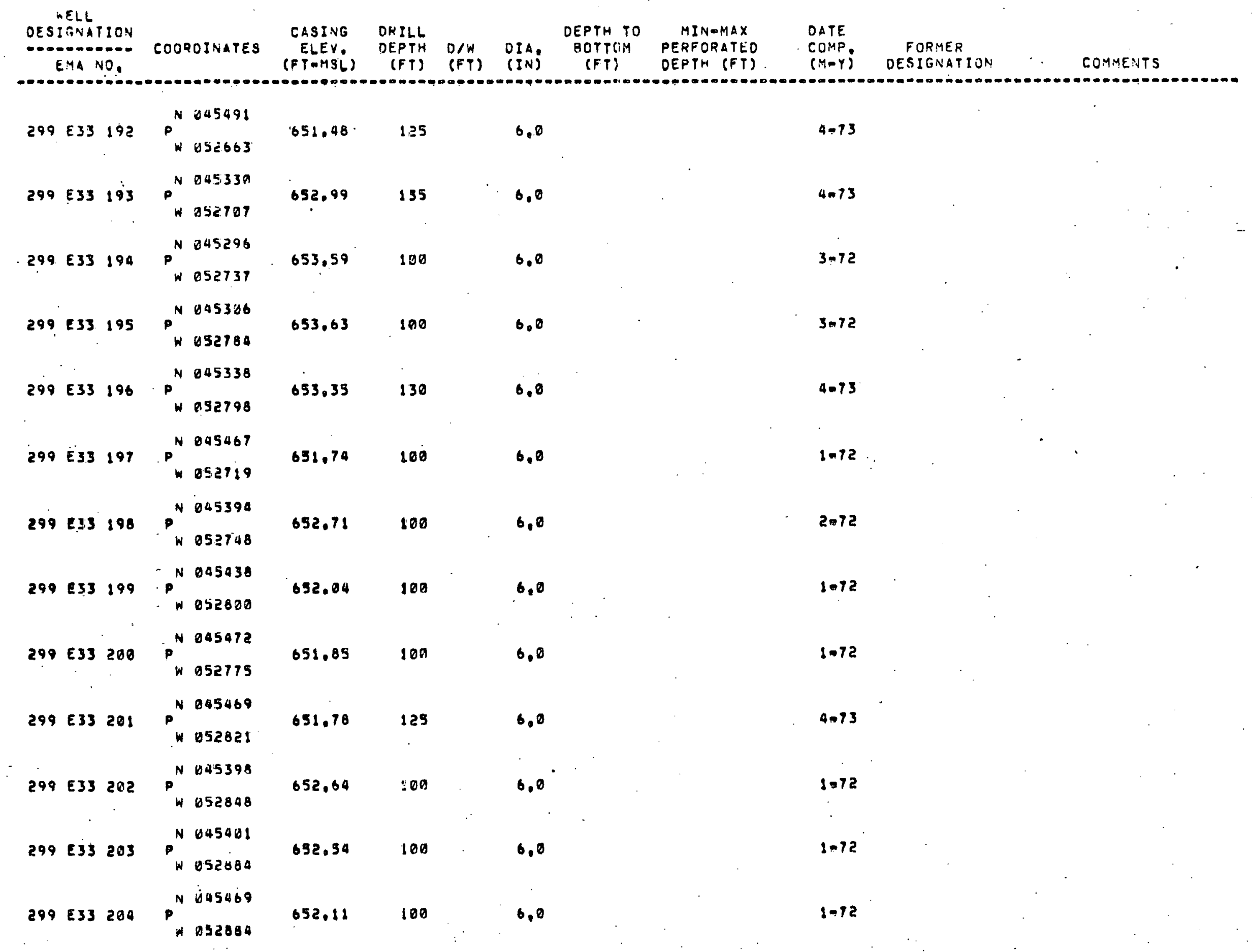


WELL

OESIGNATION

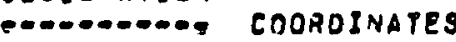

EMA NO.

CASING ORILL. DEPTH TO MINAMAX

BOTTOM PERFORATED

DATE

(ETOMSL) (FT) (FT) (IN) (FT) OEPTH (FT)

COMP. FORMER

$(M-Y)$ DESIGNATION

299633205

0.0

$299 . E 33226$

649.52

100

6,0

$1-73$

$299 E 33207$

608,84

100

6.0

1073

$299 E 33208$

648.55

100

6.0

$1=73$

$299 E 33209$

109

6.0

1.73

$259 E 33210$

100

6.0

12.72

$299 \quad 533211$

648.01

100

6.2

$12-12$

$299 E 33212 \cdot p^{\text {N }} 045257$

$654.25 \quad 100$

6,0

$8-73$

$299 E 33213-P^{N} 045257$
W 052819.

$554.02-100-0.0$

8.73

$299 E 33214 P^{N 04528 \pi}$

$\$ 54.27 \quad 180$

6,0

8.73

$299 E 33215 P^{N} 445238$

$\$ 55.15 \quad 135$

$299 E 33216$ N 045230

$\$ 55.49 \quad 100$

$$
6,0
$$

9.73

$299 E 33217 P^{N} 1045337$

$553,77 \quad 129$

6,0

$5=73$ 
WELL

DESIGNATION

EMA NO.

COOROINATES

CASING ORILL

DEPTH TO MIN-MAX

BOTTEIM

PERFORATEO

DEPTH (FT)

DATE

(M-Y) DESIGNATION

DEIGNATION . COMMENTS

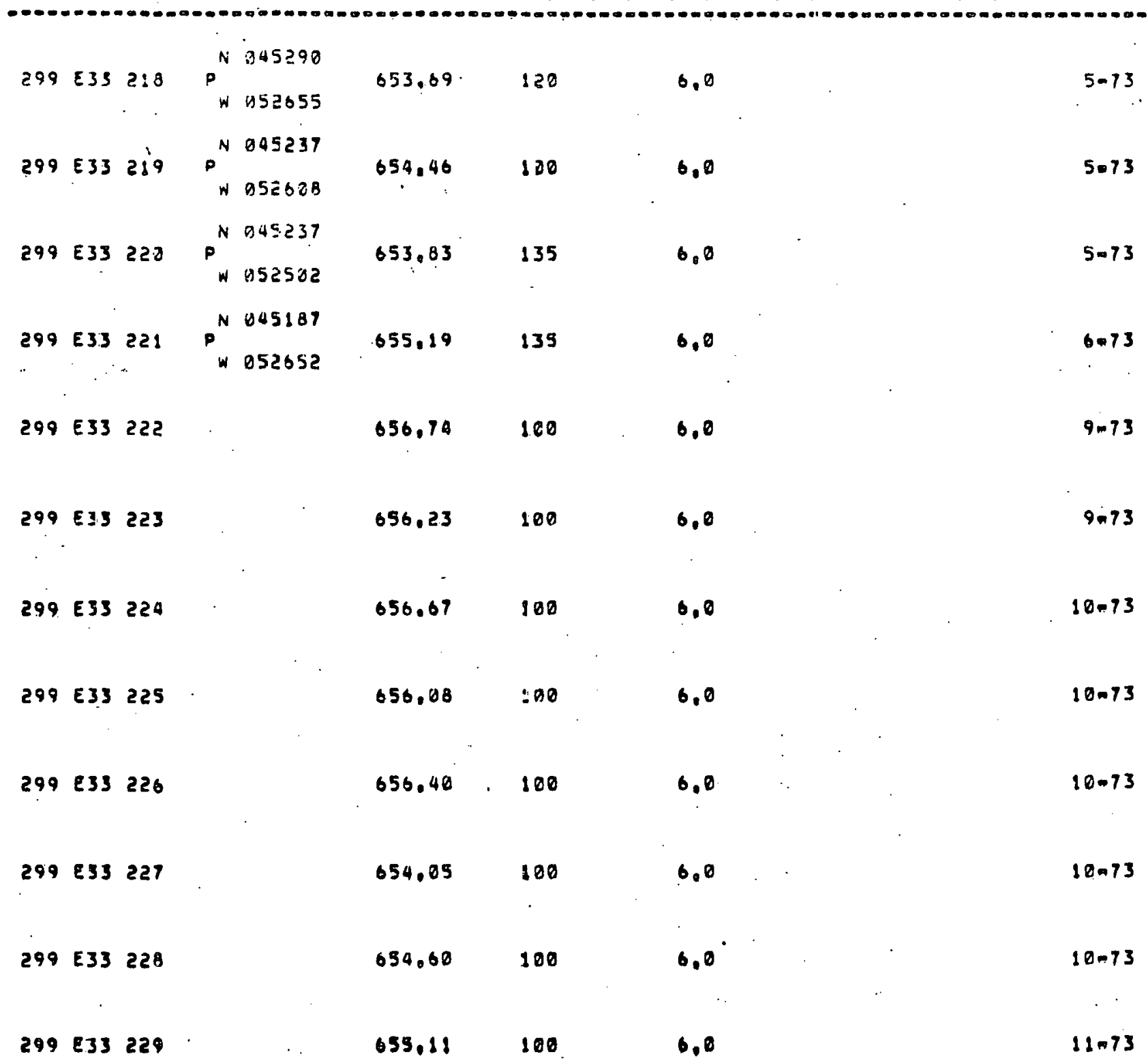

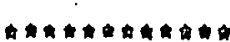




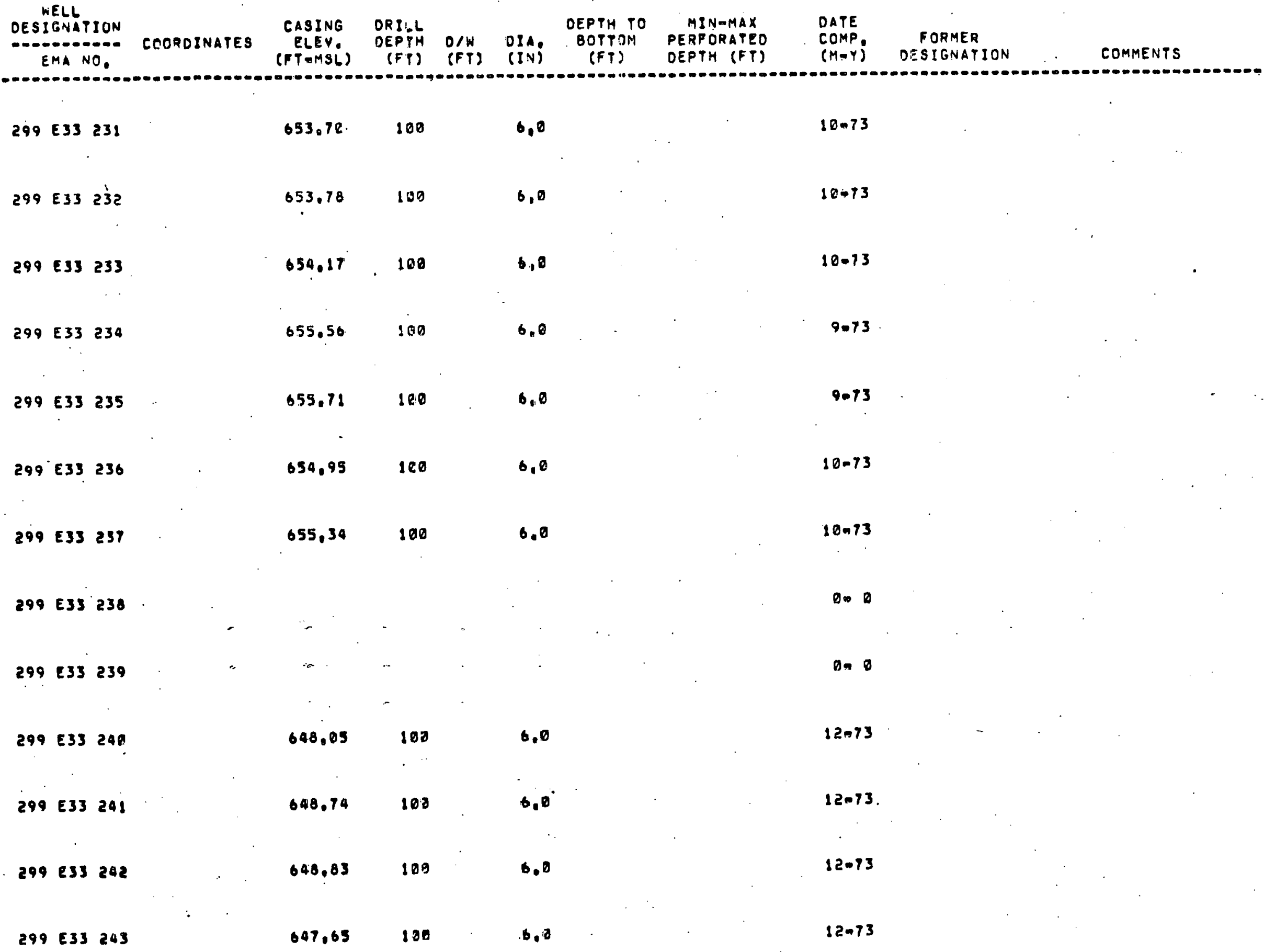


WELL

DESIGNATION

e-coma no.

EMA NO.

COOROJNATES

CASING

ELEV.

DRILL

DEPTH TO MINGMAX

BOTTCM PERFORATED

DEPTH (FT)

DATE

COMP, FORMER

(M-Y) OESIGNATION

COMMENTS

$29.9 E 33240$

698.76

100

6,0

$12-73$

$299 E 33 \quad 245$

100

6.0

5.74

$299 E 33246$

100

6,0

5.74

$299 \quad E 33 \quad 247$

$0=0$

$299 E 13208$

100

6.0

5.74

$299 E 33 \quad 249$

100

6,0

$5-74$

$299 E 33250$

100

6.0

$5-74$

$299 E 3325$

100

6.0

$5 n 74$

$299 E 33252$

$: 00$

6.0

$5+74$

$299 \quad 533 \quad 253$

180

6.0

5.74

$299 E 33254$

180

6.0

$5=74$

$299 E 33 \quad 255$

100

6,0

$5=70$

299 E33. 296

100

6.0

$5-74$ 
WELL

DESIGIVATION oestgivation EMA NO.

CASING

DRILL

DEPTH TO
DEPTH O/W OIA. BOTTOM

ELEV.

(FT) (FT) (I.V) (FT)

MINGMAX
PERFORATED

DATE DEPTH (FT)

COMP.

$(M-Y)$

FORMER

OES IGNATION

$299 E 33257$

653.93

109

6,0

$12 \div 73$

$299 E 33258$

654.25

100

6.0

12.73

$299 E 33259$

100

6.0

$6=74$

$299 E 33269$

100

6.0

6.74

$299 E 33261$

100

$b, 0$

6070

$299 \quad 533 \quad 262$

100

6,0

6.74

$299 E 35263$

100

6,0

$5 \cdot 74$

$299 \quad 533264$

100

6.8

$6-74$

$299 E 30 \quad 1 \quad p^{N-045129}$

629.42

$245 \quad 225$

0.245

6061 


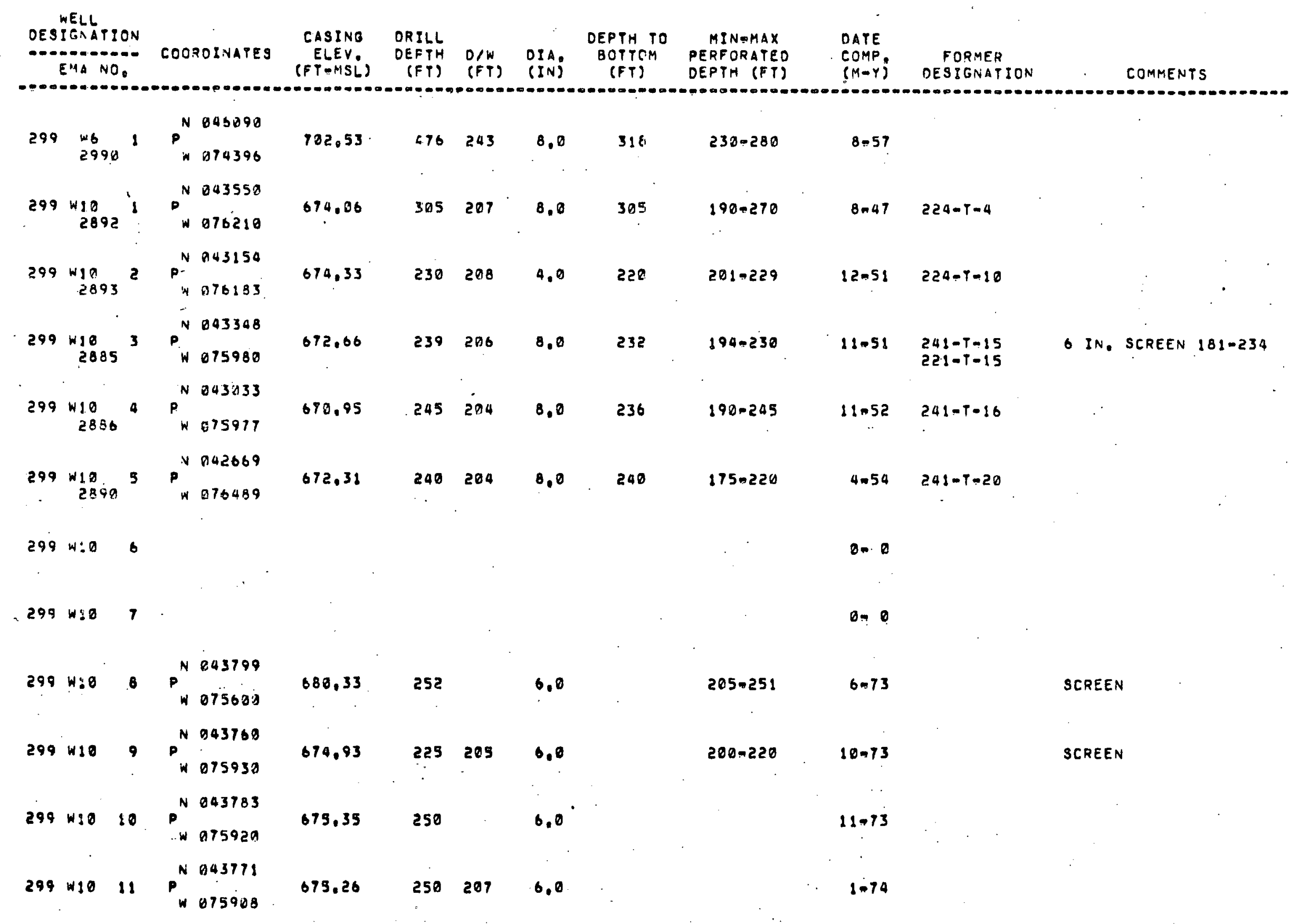




\begin{tabular}{|c|c|c|c|c|c|c|c|c|c|c|c|}
\hline OESI & $\begin{array}{l}\text { ELL } \\
\text { IGNAT } \\
\text { MAA }\end{array}$ & & COOROINATES & $\begin{array}{c}\text { CASINS } \\
\text { ELEV. } \\
\text { (FT=MSS-) }\end{array}$ & $\begin{array}{l}\text { ORILL } \\
\text { DEPYH } \\
\text { (FT) }\end{array}$ & $\begin{array}{l}\text { O/W } \\
\text { SPT: }\end{array}$ & $\begin{array}{l}\text { OIA. } \\
\text { IINI }\end{array}$ & $\begin{array}{l}\text { DEPTH TO } \\
\text { BOPTIIM } \\
\text { (FT) }\end{array}$ & $\begin{array}{l}\text { MINAMAX } \\
\text { PERFORATED } \\
\text { OEPTH (FT) }\end{array}$ & $\begin{array}{l}\text { DATE } \\
\text { COMP. } \\
\text { CM-Y) }\end{array}$ & $\begin{array}{c}\text { FORMER } \\
\text { OESIGNATIO }\end{array}$ \\
\hline 299 & W10 & 12 & $\begin{array}{l}P^{N} 043753 \\
N 075906\end{array}$ & 675.00 & & & & & & O. 0 & \\
\hline 299 & 10 & .51 & $\begin{array}{l}P_{W}^{N} 043484 \\
P^{2} 079774\end{array}$ & 671.55 & 148 & & 6,0 & 149 & $50=150$ & 10.44 & $241-T-131$ \\
\hline 299 & WIO & 52 & $\begin{array}{l}N 043385 \\
P^{N} \quad 075408\end{array}$ & 672.11 & 148 & & 0.8 & 149 & $58-150$ & 10.44 & $241-T-134$ \\
\hline 290 & N10 & 53 & $\begin{array}{l}F^{N} 043711 \\
W 075714\end{array}$ & 6.71 .98 & 151 & & 6.0 & 150 & $50-150$ & 10.44 & $241=T-130$ \\
\hline 299 & W10 & 50 & $\begin{array}{l}\text { N } 043584 \\
W 075907\end{array}$ & 671.61 & 150 & . & $b .0$ & 148 & $50-150$ & 10.44 & $241-T-132$ \\
\hline 299. & $w 10$ & 55 & $P^{N 043271}=75700$ & 672.34 & 150 & - & $1 \geq .0$ & 149 & $50-150$ & 9.44 & $241-T-136$ \\
\hline 299 & $W 10$ & 56 & $\begin{array}{l}P^{N} 843434 \\
\text { W } \\
070003\end{array}$ & 672.86 & 139 & & 0.0 & 150 & & 6.47 & $224-T-1$ \\
\hline 299 & W10. & 57 & $P^{N 043449}$ & 673.99 & 150 & & 0,0 & 150 & & $6=47$ & $2 \approx 4-7-2$ \\
\hline 299 & w10 & 58 & $\begin{array}{l}P^{V 043418} \\
N 075994\end{array}$ & 672.46 & 150 & & 8.8 & 150 & & 7.47 & $224-7-3$ \\
\hline 299 & W10 & 59 & $\begin{array}{l}P^{N} 043298 \\
N 070012\end{array}$ & 672.24 & 1.50 & . & 8.0 & 150 & $33-38$ & $7-47$ & $\begin{array}{l}241-T=2 \\
221-T=2\end{array}$ \\
\hline 299 & W1a & 60 & $\begin{array}{l}P^{N} 043298 \\
.075998\end{array}$ & 671.94 & 150 & & 8.0 & 150 & $31 \cdot 36$ & .7 .47 & $\begin{array}{l}241-T=3 \\
221-T=3\end{array}$ \\
\hline 299 & wio & 61 & $\begin{array}{l}P^{N 045272} \\
W 070012\end{array}$ & 672,29 & 150 & & 0.0 & 150 & $32 \cdot 37$ & $7=47$ & $\begin{array}{l}241=T-5 \\
221=T-5\end{array}$ \\
\hline 299 & $w ! 0$ & 62 & $\begin{array}{l}P^{N} 843305 \\
\end{array} 976020$. & 672.37 & $15 \pi$ & & 8,0 & 158 & & $7-47$ & $\begin{array}{l}241-T-6 \\
221=T-6\end{array}$ \\
\hline
\end{tabular}




\begin{tabular}{|c|c|c|c|c|c|c|c|c|c|c|c|}
\hline $\begin{array}{r}\text { OESI } \\
- \\
-\end{array}$ & $\begin{array}{l}\text { NEL } \\
\text { GNAT } \\
M A N \\
M A\end{array}$ & $\begin{array}{l}\text { ION } \\
0 . \\
0 .\end{array}$ & COORUINATES & $\begin{array}{c}\text { CASING } \\
\text { ELEV } \\
\text { (FT-MSL) }\end{array}$ & $\begin{array}{l}\text { DKILL } \\
\text { DEPTH } \\
\text { (FT) }\end{array}$ & $\begin{array}{l}0 / W \\
\text { (FT) }\end{array}$ & OIA; & $\begin{array}{l}\text { OEPTH TO } \\
\text { BOTTCM } \\
\text { (FT) }\end{array}$ & $\begin{array}{l}\text { MIN-MAX } \\
\text { PERFURATED } \\
\text { OEPYH (FT) }\end{array}$ & $\begin{array}{l}\text { OATE } \\
\text { COMP } \\
\text { (MAY) }\end{array}$ & $\begin{array}{c}\text { FORMER } \\
\text { DESIGNAT }\end{array}$ \\
\hline 299 & 410 & 63 & $\begin{array}{l}P_{N}^{N} 043265 \\
N 075980\end{array}$ & 671.92 & 150 & & 8.9 & 150 & & 9.47 & $\begin{array}{l}241-T-8 \\
221-T-8\end{array}$ \\
\hline 299 & 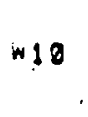 & 64 & $\begin{array}{l}\text { N } 043019 \\
W 075091\end{array}$ & 672.34 & 73 & & 8.0 & 75 & & 7.47 & $224-T-5$ \\
\hline 299 & W1O & 65 & $\begin{array}{l}\text { i. } \\
\text { p. } \\
\text { W } 045980\end{array}$ & 673.87 & 79 & & 8.0 & 75 & . & $8 \times 47$ & $224-T-6$ \\
\hline 299 & 12 & 66 & $\begin{array}{l}P_{W}^{N} 045272 \\
W 075980\end{array}$ & 671,80 & 125 & & 8.0 & 129 & $31=36$ & 9048 & $\begin{array}{l}241-T=4 \\
221=T-4\end{array}$ \\
\hline 299 & W10 & 67 & $\begin{array}{l}P^{N} 263305 \\
N 075980\end{array}$ & 672.34 & 150 & & 8.0 & 150 & & 8.47 & $\begin{array}{l}24 !-T-7 \\
221-T-7\end{array}$ \\
\hline 299 & W18 & 68 & $\begin{array}{l}P^{N} 043265 \\
W 076020\end{array}$ & 672.13 & 850 & & 8.0. & 158 & & 8.47 & $\begin{array}{l}241-T=9 \\
221-T-9\end{array}$ \\
\hline 299 & $w 10$ & 69 & $\begin{array}{l}P_{W}^{N} 043320 \\
W 076110\end{array}$ & 673.44 & 150 & & 8,0 & 150 & . & $8=47$ & $\begin{array}{l}241-T-10 \\
221-T=10\end{array}$ \\
\hline 299 & $w 10$ & 10 & $\begin{array}{l}\rho^{N} 043342 \\
W 096272\end{array}$ & 673.84 & 1.50 & & 8,0 & 150 & & 8.47 & $\begin{array}{l}241-T-11 \\
221-T-11\end{array}$ \\
\hline 299 & $w 10$ & 71 & $\begin{array}{l}P^{N 043285} \\
+076317\end{array}$ & 673.98 & .150 & & 8,0 & 150 & $60 \cdot 80$ & $8 m 47$ & $\begin{array}{l}241-T-12 \\
221-T-1 \cdot 2\end{array}$ \\
\hline 299 & $W: 8$ & 12 & $\begin{array}{l}\rho^{N .043270} \\
W 076200\end{array}$ & 673.01 & 150 & & 8,0 & 150 & & $8=47$ & $\begin{array}{l}241-T-13 \\
221-T-13\end{array}$ \\
\hline 299 & $W 10$ & 93 & $\begin{array}{l}P^{N} 043364 \\
W 016082\end{array}$ & 673.83 & 7.5 & & 8,0 & 75 & & $9=47$ & $\begin{array}{l}241-T-14 \\
221-T-14\end{array}$ \\
\hline $299^{\circ}$ & $w_{10}$ & 90 & $\begin{array}{l}P_{N}^{N} 043285 \\
N 076000\end{array}$ & 672.81 & $49^{\circ}$ & & 8.8 & 49 & & $10=47$ & $\begin{array}{l}241-T-1 \\
221-T-1\end{array}$ \\
\hline 299 & N10. & 75 & $\begin{array}{l}P^{N} 043431 \\
\text { in } 8759480\end{array}$ & 674.71 & 95 & . & 8.0 & 75 & & 10.47 & $224-9-7$ \\
\hline
\end{tabular}


WELL

OESIGNATION COOROINATES

$\begin{array}{ll}\text { CASING DRILL } & \text { DEPTH TO MINAMAX } \\ \text { ELEV DEPTH DIH DIA BOTTEM PERFORATEO }\end{array}$

BOTTEM

PERFORATEO

DATE

EMA NO,

(FTAMSL) (FT) (FT) (IN) (FT) DEPTH (FT)

$(M-Y)$

FORMER

DESIGNATION

COMMENTS

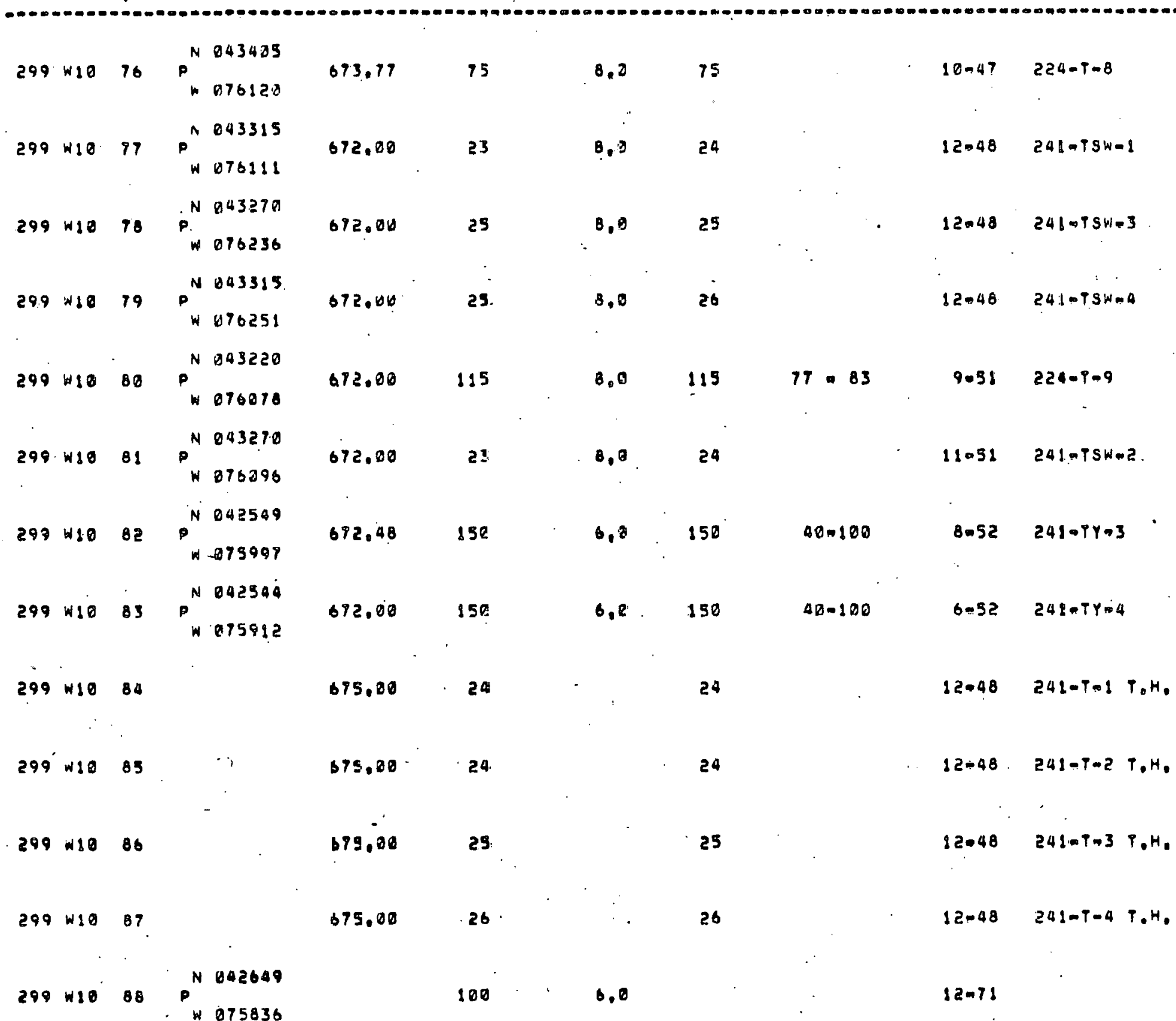


WELL

DESIGNATIO

EMA ND.

COORDINATES

CASING ORILL

$\begin{array}{lll}\text { CASING ORILL } & & \text { DEPTH TO MINAMAX } \\ \text { ELEV. DEPTH D/N DIA. BOTTEM PERFURATED }\end{array}$

DATE (FT-MSL) (FT) (FI) (IN) (FT) DEPTH (FT)

FORMER

DESIGNATION

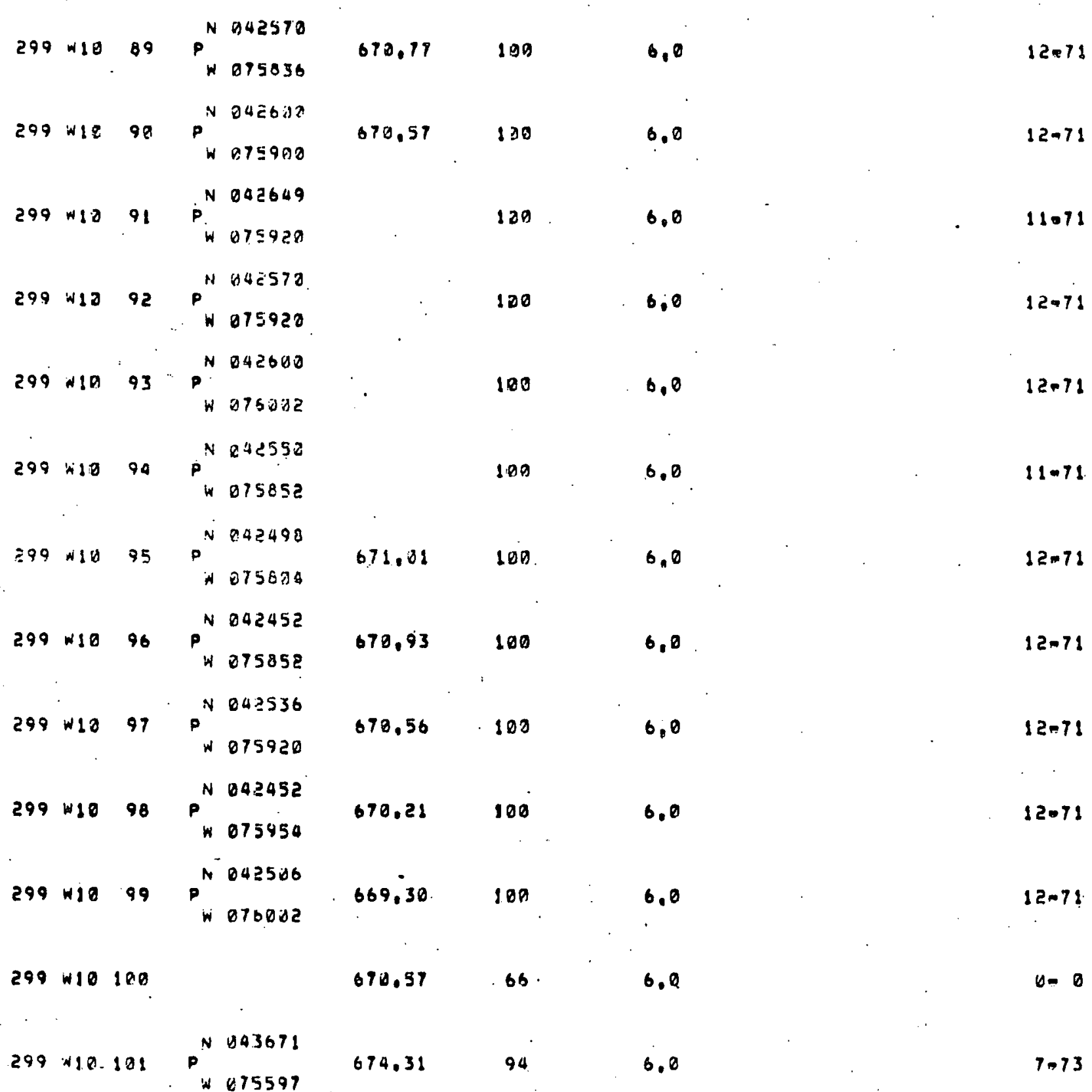




\begin{tabular}{|c|c|c|c|c|c|c|c|c|c|c|}
\hline DES: & $\begin{array}{l}\text { WELL } \\
\text { IGNAT } \\
\text { EMA }\end{array}$ & $\begin{array}{l}\text { igOn } \\
\text { ino. }\end{array}$ & COUROIVATES & $\begin{array}{c}\text { CASING } \\
\text { ELEV. } \\
\text { (FT:MSL) }\end{array}$ & $\begin{array}{l}\text { DKILL } \\
\text { DEPTH } \\
\text { (FT) }\end{array}$ & $\begin{array}{l}D / W \\
\text { (FT) }\end{array}$ & $\begin{array}{l}\text { DIA } \\
\text { (IN) }\end{array}$ & $\begin{array}{l}\text { DEPTH TO } \\
\text { BOTTIM } \\
\text { (FT) }\end{array}$ & $\begin{array}{l}\text { MIN-MAX } \\
\text { PERFORATED } \\
\text { DEPTH (FY) }\end{array}$ & $\begin{array}{l}\text { OAPE } \\
\text { COMP } \\
\text { (M=Y) }\end{array}$ \\
\hline 299 & W10 & 102 & $\begin{array}{l}P^{N} 043632 \\
W 075595\end{array}$ & 674.18 & 87 & & 3.0 & & & 8.73 \\
\hline 299 & 118 & 10.3 & $\begin{array}{l}P^{N} 043593 \\
\text { HN } 075637\end{array}$ & 673.04 & 94 & & $b, 0$ & & & $8-73$ \\
\hline 2.99 & $w 10$ & 104 & 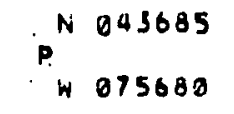 & 613.17 & 96 & & 6.0 & & . & $8 n+3$ \\
\hline 299 & $W 10$ & 105 & $\begin{array}{l}P^{2} 043692 \\
N 075637\end{array}$ & & द2 & & 6,0 & & & 7.73 \\
\hline 299 & $W 10$ & 186 & $\begin{array}{l}\text { iN } 043582 . \\
\text { N075837 }\end{array}$ & 761.42 & 120 & & 6,0 & & & 7.73 \\
\hline 299 & $w 10$ & 187 & $\begin{array}{l}\text { N043539 } \\
\text { P } \\
W 075792\end{array}$ & 671.91 & 100 & & $6,0$. & & & $7-73$ \\
\hline 299 & $w 10$ & 108 & $\begin{array}{l}P_{H}^{N 043580} \\
+075805\end{array}$ & 671.84 & 92 & & 0.0 & & & 9.73 \\
\hline 299 & w10 & 109 & 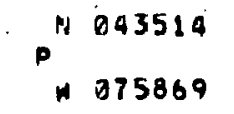 & 671.10 & 92 & & 6.0 & & & $7=73$ \\
\hline 299 & $w 13$ & 110 & $\begin{array}{l}p^{M} 043531 \\
h 075740\end{array}$ & 671.30 & 93 & & 6,0 & & . & 7.73 \\
\hline 299 & w10 & 111 & $\begin{array}{l}P^{N} 043511 \\
N 075829\end{array}$ & 671.37 & 94 & & 6,0 & & & $8=73$ \\
\hline 299 & W10 & 112 & $\begin{array}{l}P_{N}^{N} 043452 \\
N 075787\end{array}$ & 671.76 & 81 & & 6.7 & & & $7-73$ \\
\hline 299 & W10 & 113 & $\begin{array}{l}P_{W}^{N} 043502 \\
{ }^{2} 075742\end{array}$ & 671.61 & 8. & & - & & & 0.77 \\
\hline 299 & $\$ 10$ & 114 & $\begin{array}{c}P^{N} 84380 \\
W 675870 \\
\ddots\end{array}$ & 671,26 & 93 & & 5,0 & & & 7.73 \\
\hline
\end{tabular}




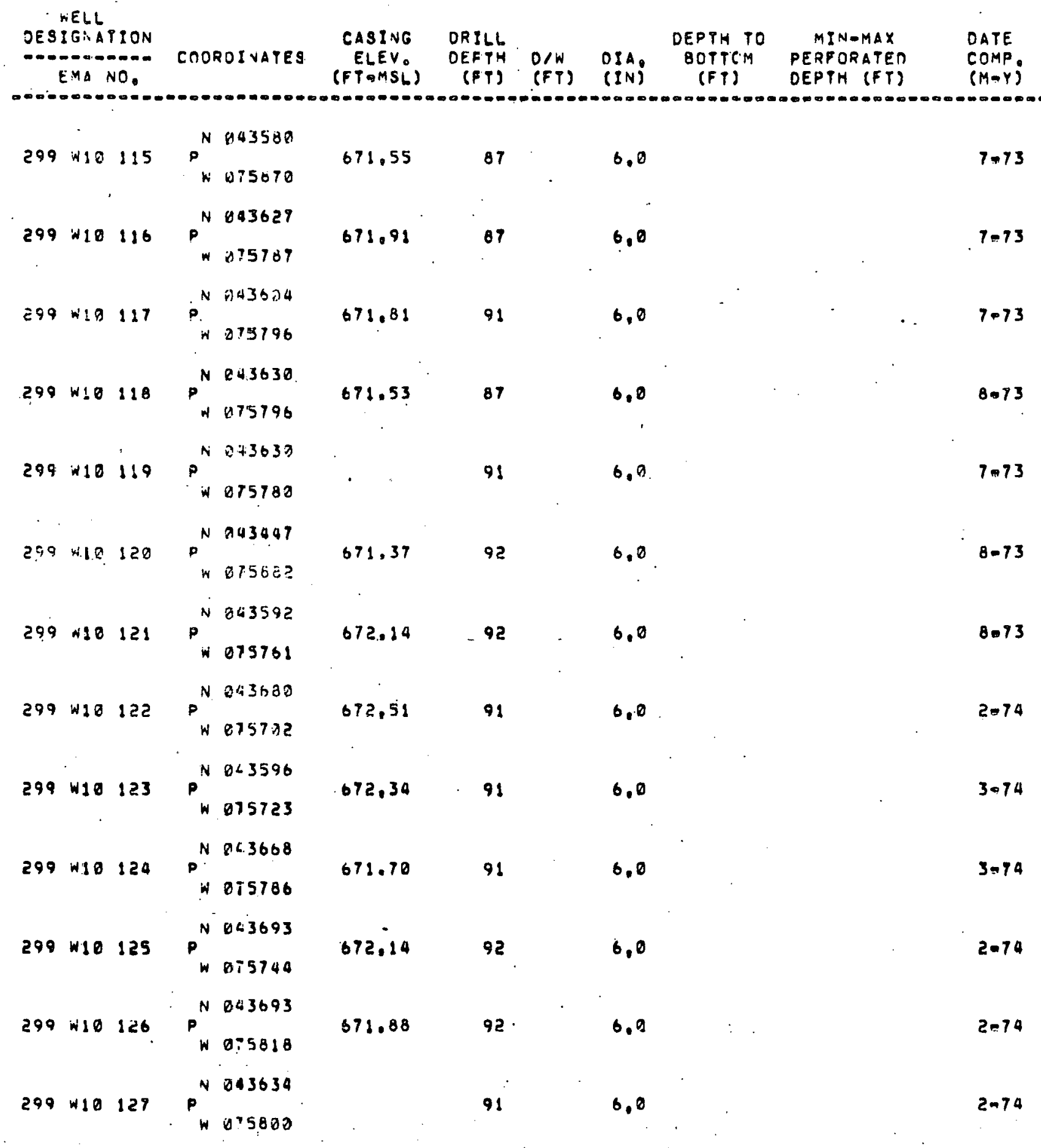


WELL

DESIGNATION

EMA NO.

COOROIVATES

CASING

ELEV.

DRILL
DEPTH

O/W DIA, DEPTHTO

$M I N-M A X$

PEPFORAPED

DATE (F P-MSL)

(PT) (FT) (IV)

(FT)

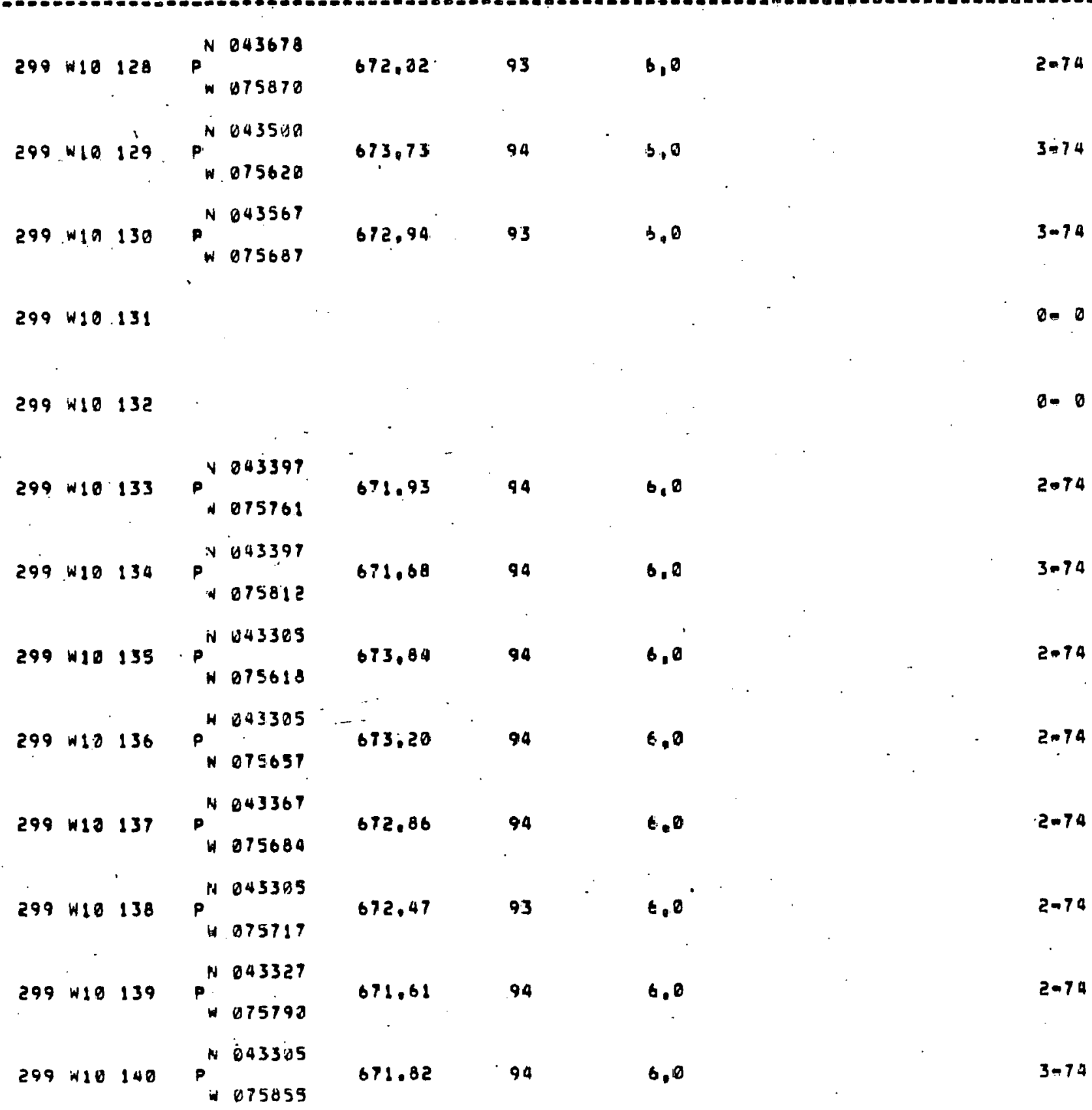


ELL

OESIGNATION

Ema nOa

COOROJNATES

CASIVE DKILL

DEPTH TO MINAMAX
DOTTUM PERFURATEO

DEPTH (FT)

DATE

(FTAMSL) (FT) (F.T) (IN)

FORMER

DESIIGNATION

COMMENTS

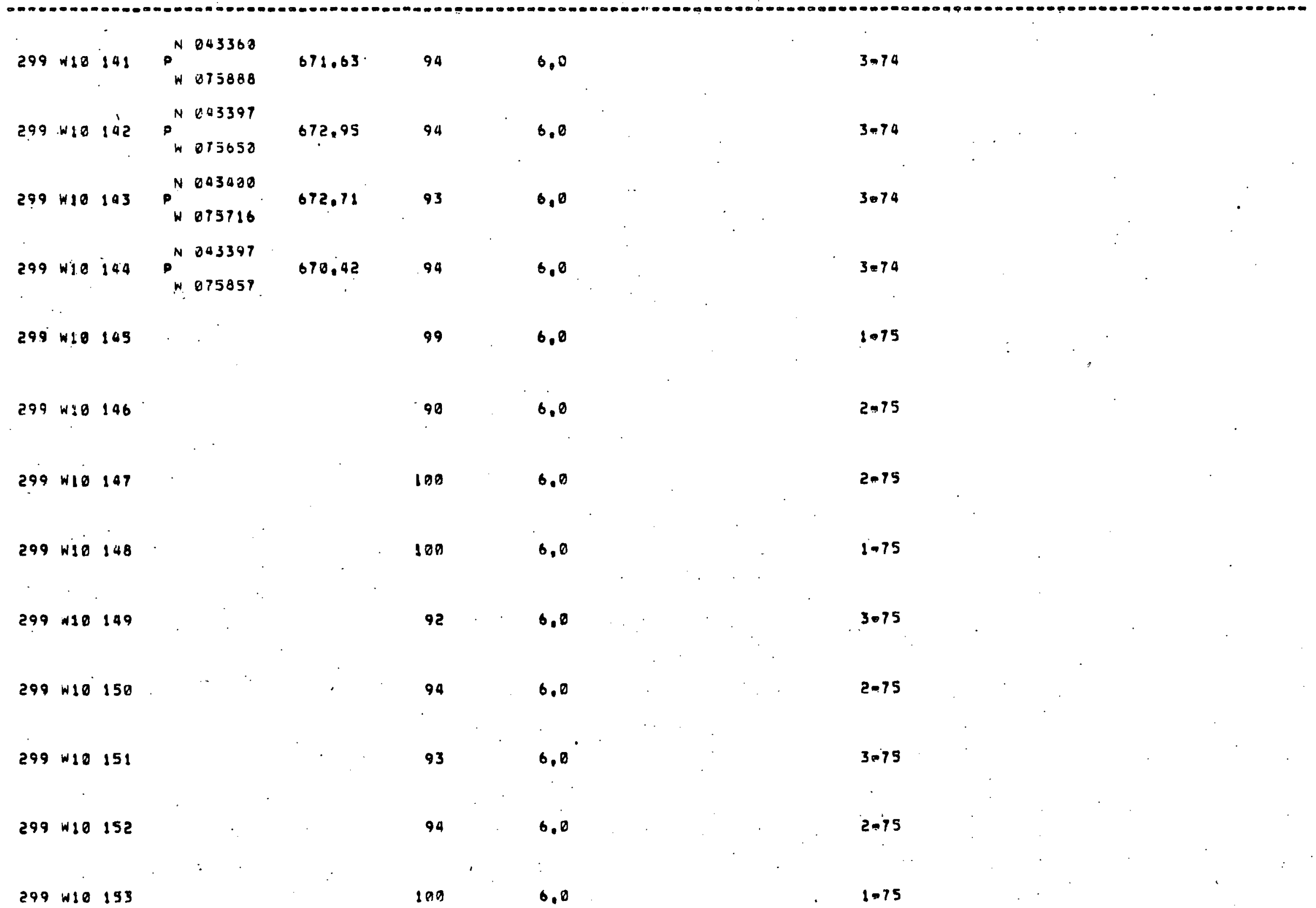




\begin{tabular}{|c|c|c|c|c|c|c|c|c|c|c|c|c|c|}
\hline OES & $\begin{array}{l}\text { IGNATIO } \\
\text { EANONO. }\end{array}$ & & COORUINATES & $\begin{array}{c}\text { CASING } \\
\text { ELEVY } \\
(F T=M S L)\end{array}$ & $\begin{array}{l}\text { ORILL } \\
\text { OEPTH } \\
\text { (FT) }\end{array}$ & $\begin{array}{l}0 / n \\
\text { (f T T) }\end{array}$ & $\begin{array}{l}\text { DIA. } \\
\text { CINi }\end{array}$ & $\begin{array}{l}\text { DEPTH TO } \\
\text { BOTYOM } \\
\text { (FT) }\end{array}$ & $\begin{array}{l}\text { MINAMAX } \\
\text { PERFURATEN } \\
\text { DEPTH (FT) }\end{array}$ & $\begin{array}{l}\text { DATE } \\
\text { COMP, } \\
\text { (M-Y) }\end{array}$ & $\begin{array}{l}\text { FORMER } \\
\text { DESIGNATION }\end{array}$ & . & COMME \\
\hline 299 & W10 15 & & & . & 100 & & 6.0 & & & 1.75 & & & \\
\hline 299 & $\begin{array}{l}W 11 \\
2873\end{array}$ & 1 & $P^{N 003274}$ & 727,24 & 243 & 250 & 8,0 & 270 & $220-311$ & $3 \sin 50$ & $361-9-12$ & & \\
\hline 299 & $\begin{array}{l}\text { W11 } \\
2879\end{array}$ & 2 & $\begin{array}{l}P_{W}^{N} 043335 \\
W 3772\end{array}$ & 714.51 & 530 & 253 & 8,0 & 259 & $256-508$ & $11-50$ & $361-9-14$ & & \\
\hline & & & 0 & 714.75 & 259 & 252 & 1,5 & & $239-259$ & $11-62$ & & & \\
\hline & 2601 & & P & 714,75 & 508 & 254 & 1.5 & 498 & $486-508$ & $11-62$ & & & - \\
\hline & 2602 & & 0 & 714.75 & 434 & & 1,5 & 434 & $499=434$ & 11.62 & & & \\
\hline & 2603 & & $\mathbf{R}$ & 714.75 & 380 & & 1.5 & 386 & $358-380$ & $11-62$ & & & . \\
\hline$\therefore$ & 2600 & & $\mathbf{s}$ & 714.75 & 322 & & 1,5 & 322 & $300-322$ & $11-62$ & & & \\
\hline & 2605 & & $\mathbf{T}$ & 714,75 & 264 & & 1,5 & 261 & $242-264$ & $11-62$ & & & \\
\hline 2.99 & $\begin{array}{l}111 \\
2989\end{array}$ & 3 & $\begin{array}{l}P^{N 043310} \\
N 073801\end{array}$ & 718.46 & 321 & 259 & 8.8 & 318 & 254.320 & 1051 & $361-T-15$ & 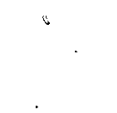 & \\
\hline 299 & $\begin{array}{l}w 11 \\
2876\end{array}$ & 4 & $\begin{array}{l}\dot{p}^{4} 843068 \\
\quad 073909\end{array}$ & $7: 3.37$ & 310 & 251 & 8.8 & 310 & $258-300$ & $5-51$ & $361-7-16$ & & \\
\hline 299 & $\begin{array}{l}W 11 \\
2877\end{array}$ & 5 & $\begin{array}{l}P^{N} 043675 \\
\times 073650\end{array}$ & 917.68 & 316. & 256 & 8,0 & 310 & $257-392$ & 7.51 & $361-7-17$ & & \\
\hline 299 & $\begin{array}{l}\text { W11 } \\
2878\end{array}$ & 6 & $\begin{array}{l}P_{N}^{N} 042730 \\
N 073525\end{array}$ & 716.23 & 312 & 254 & 8,0 & 304 & $262 m 312$ & 7.51 & $361-7-18$ & & . \\
\hline 299 & $\begin{array}{l}w 11 \\
2879\end{array}$ & 1 & $\begin{array}{l}P^{N} 043350 \\
.074251\end{array}$ & 789.11 & 315 & 246 & 8.0 & 306 & $245=290$ & $9-51$ & $361-7-19$ & 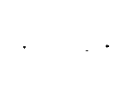 & \\
\hline 299 & $\begin{array}{l}W 11 \\
2880\end{array}$ & 8 & $\begin{array}{l}\text { P. } 042759 \\
* 072992\end{array}$ & 719.18 & 315 & 260 & 8,0 & $3: 3$ & $260-310$. & 12.53 & $361-T-22$ & & \\
\hline 2 & $\begin{array}{ll}11 \\
2881\end{array}$ & 9 & $P_{W}^{N} 872542$ & 722.94 & 297 & 264 & 8,8 & 282 & $270-292$ & 4.54 & $361-T-23$ & $6 I N_{0}$ & LINER \\
\hline
\end{tabular}




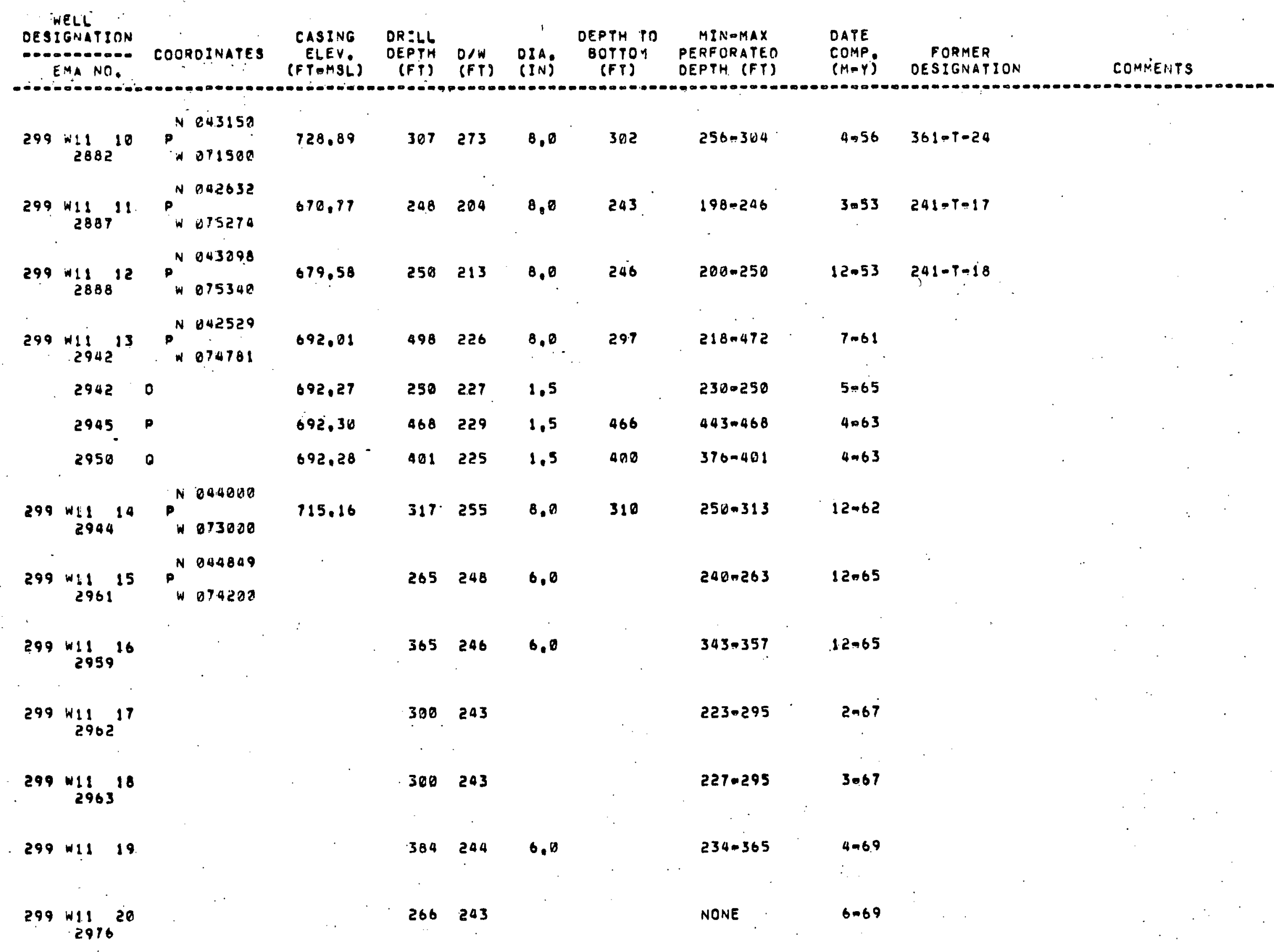




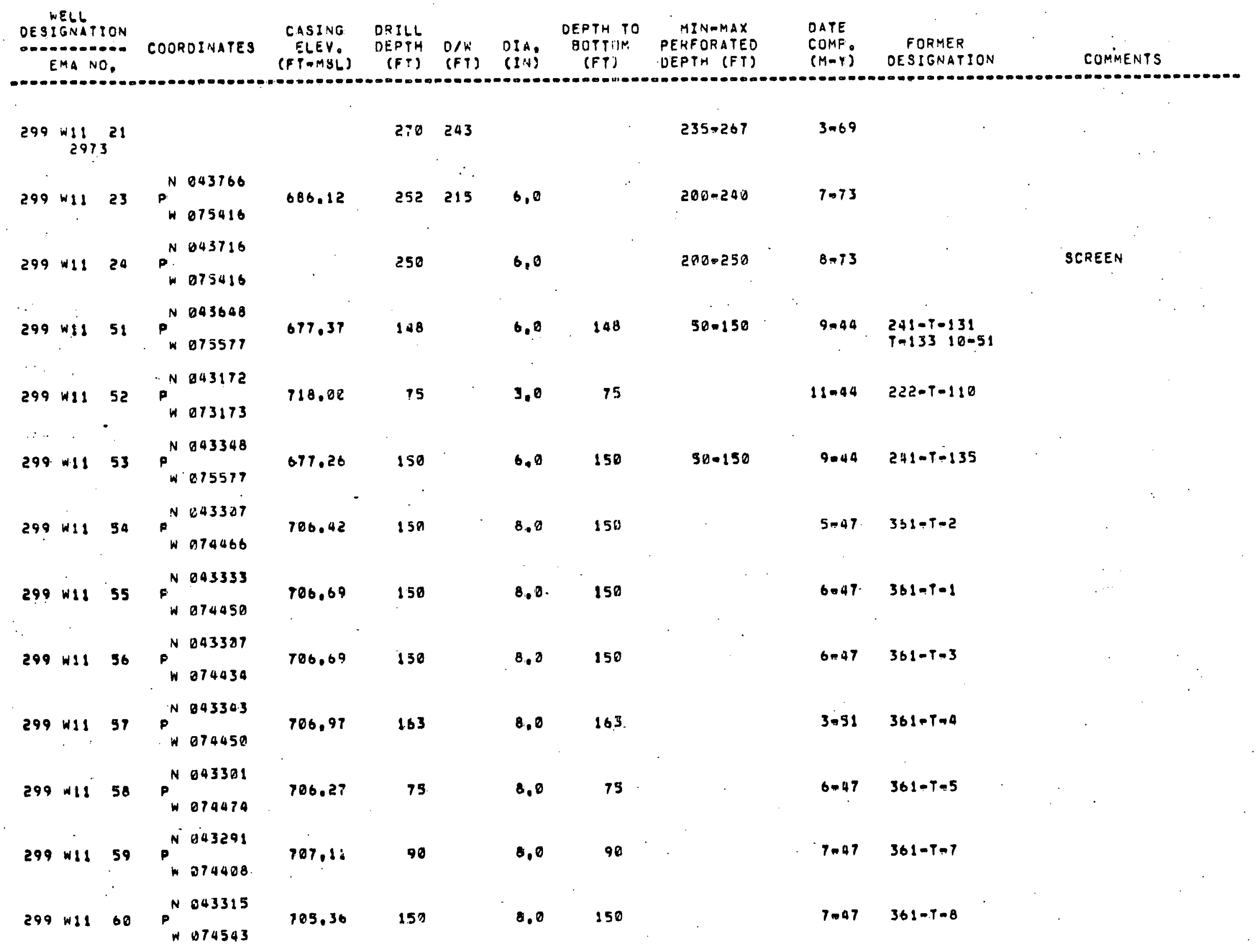




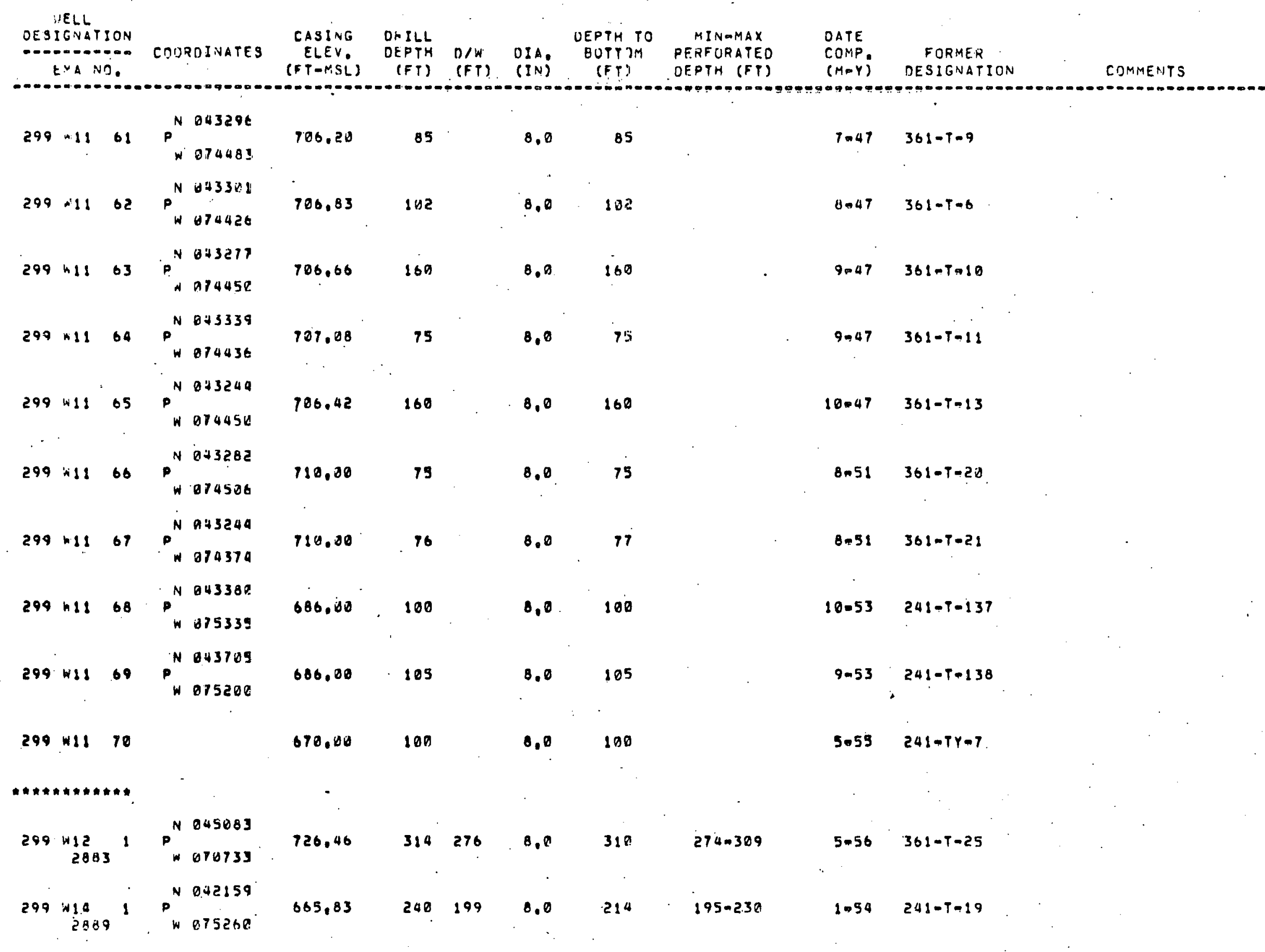




\begin{tabular}{|c|c|c|c|c|c|c|c|c|c|c|c|}
\hline OES & $\begin{array}{l}\text { WELL } \\
\text { IGNATI } \\
\text { EMA NO }\end{array}$ & & CCOROINATES & $\begin{array}{c}\text { CASING } \\
\text { ELEV. } \\
\text { (FTPMSL) }\end{array}$ & $\begin{array}{l}\text { DRILL } \\
\text { DEPTH } \\
\text { (FY) }\end{array}$ & $\begin{array}{l}0 / W \\
(F T)\end{array}$ & $\begin{array}{l}\text { DIA } \\
\text { GIVi }\end{array}$ & $\begin{array}{c}\text { OEPTH TO } \\
\text { BOTTLIMM } \\
\text { (FY) }\end{array}$ & $\begin{array}{l}\text { MIN }=M A X \\
\text { PERFORATEO } \\
\text { DEPTH (FT) }\end{array}$ & $\begin{array}{l}\text { DATE } \\
\text { COMP, } \\
(M-Y)\end{array}$ & $\begin{array}{l}\text { FORMER } \\
\text { DESIGNATION }\end{array}$ \\
\hline 299 & $\begin{array}{l}W 14 \\
2895\end{array}$ & 2 & $\begin{array}{l}P^{N} 842154 \\
W .075289\end{array}$ & 667.36 & 223 & 200 & 8.0 & 220 & $181-222$ & 5.55 & $2+1-T Y-3$ \\
\hline 290 & $\begin{array}{l}19 \\
2943\end{array}$ & 3 & $\begin{array}{l}P_{H}^{N} 0.42265 \\
H 075325\end{array}$ & 662.06 & 269 & 200 & 8.0 & 234 & $298-268$ & $12=61$ & \\
\hline & 2943 & & 0 & 6.62 .27 & 220 & 207 & 1.5 & $\cdot$ & $200-220$ & 5.65 & - \\
\hline & 2946 & & $\mathbf{p}$ & $6.62,27$ & 248 & 280 & 2.5 & 247 & $228-248$ & 5.65 & - \\
\hline 299 & $w 14$ & 4 & & & 275 & 199 & 6.0 & . & NONE & 7.66 & \\
\hline 299 & $w 14$ & 5 & & . & 240 & 205 & 6,0 & & $190-225$ & 10.74 & \\
\hline 209 & $w 14$ & 6 & & & 240 & 205 & 6.0 & & 195.225 & 12.74 & \\
\hline 299 & $w 18$ & 51 & $\begin{array}{l}p^{N} 841144 \\
W 075587\end{array}$ & 661.06 & 35 & & 8,0 & 26 & $208-268$ & $12-48$ & $271-1 \times 5 w n 2$ \\
\hline 299 & $w 14$ & 52 & $\begin{array}{l}P_{W 075587}^{N} 040934 \\
P_{1075}\end{array}$ & 061.08 & 27 & & 0.8 & $2 \varepsilon$ & $208=268$ & $12-48$ & $2+1-T \times S \operatorname{wn} 4$ \\
\hline 299 & w14 & 53 & & 6.70 .00 & 100 & & 0,0 & 100 & $288-268$ & 5.55 & $241-T Y-6$ \\
\hline 299 & $\begin{array}{l}115 \\
2897\end{array}$ & 1 & $\begin{array}{l}P_{W}^{N} 040962 \\
W 0576\end{array}$ & 672,86 & 300 & 202 & $B, \theta$ & 295 & $190-270$ & 5047 & $231-2$ \\
\hline 299 & $\begin{array}{l}\text { W15 } \\
2891\end{array}$ & 2 & $\begin{array}{l}P_{W}^{N} 042251 \\
W 970002\end{array}$ & 690.71 & $2 b 0$ & 221 & 0,0 & 253 & $218-258$ & 8.54 & $241-1-21$ \\
\hline 299 & $\begin{array}{l}w 15 \\
2894\end{array}$ & 3 & $\begin{array}{l}P_{W}^{N} 042353 \\
W 75996\end{array}$ & 671.57 & 245 & 203 & $\theta, \theta$ & 241 & $200-245$ & $9-52$ & $241-T Y=2$ \\
\hline 299 & $\begin{array}{l}\text { w15 } \\
2896\end{array}$ & a & & 662.02 & 217 & 993 & $B, \theta$ & 212 & $170-216$ & $1-56$ & $241-7 x-12$ \\
\hline
\end{tabular}




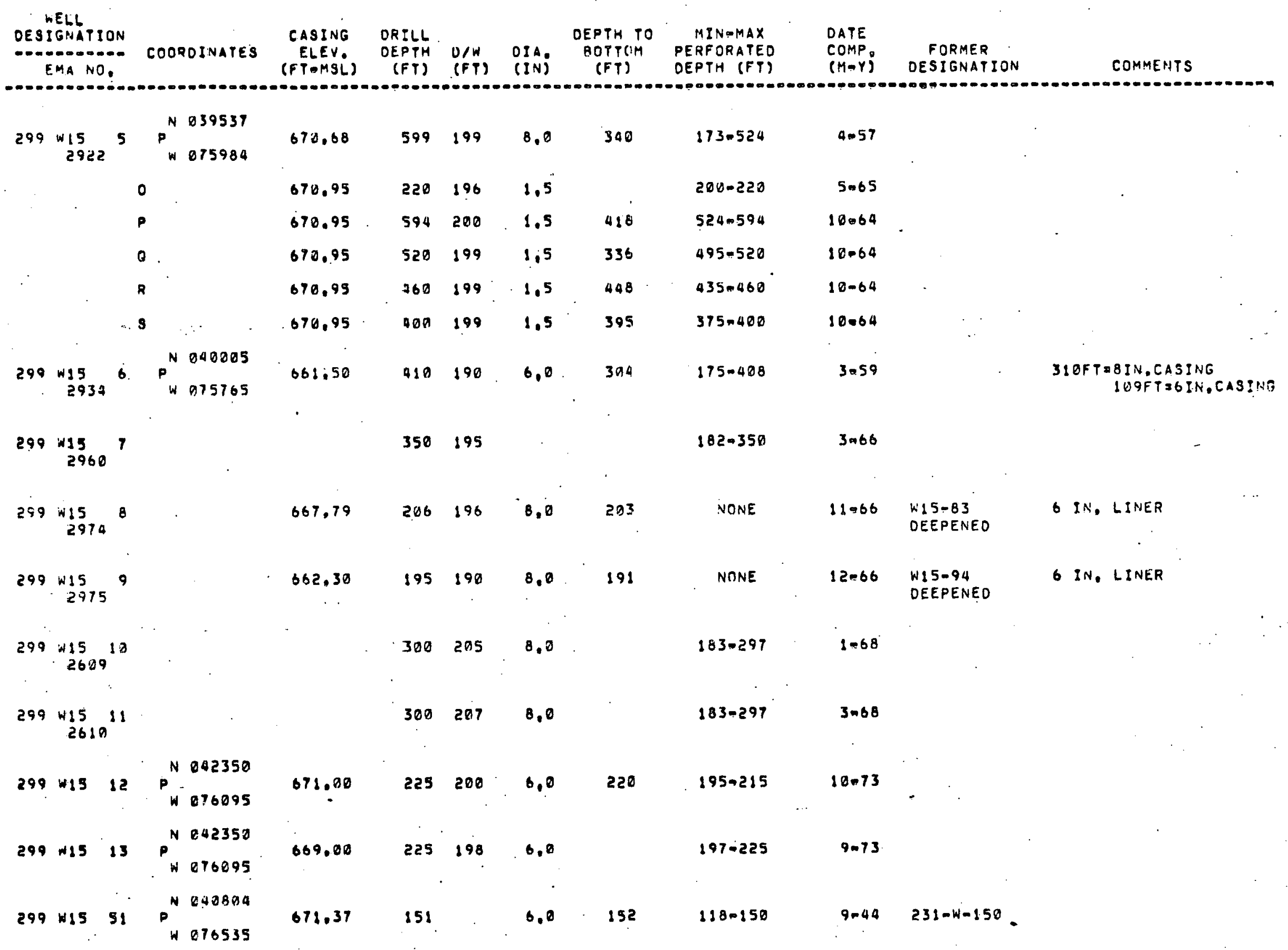


VIELL

DESIGVATION

EMA NC.

cojRuInates

CASING URILL

MINAMAX
PERFORATED

BOTPCM

OEPTH (FT)

DATE

COMP.

(FTAMSL)

(FP) (FT) (IN)

(FY)

$(M-Y)$

FORMER

DESIGNATION

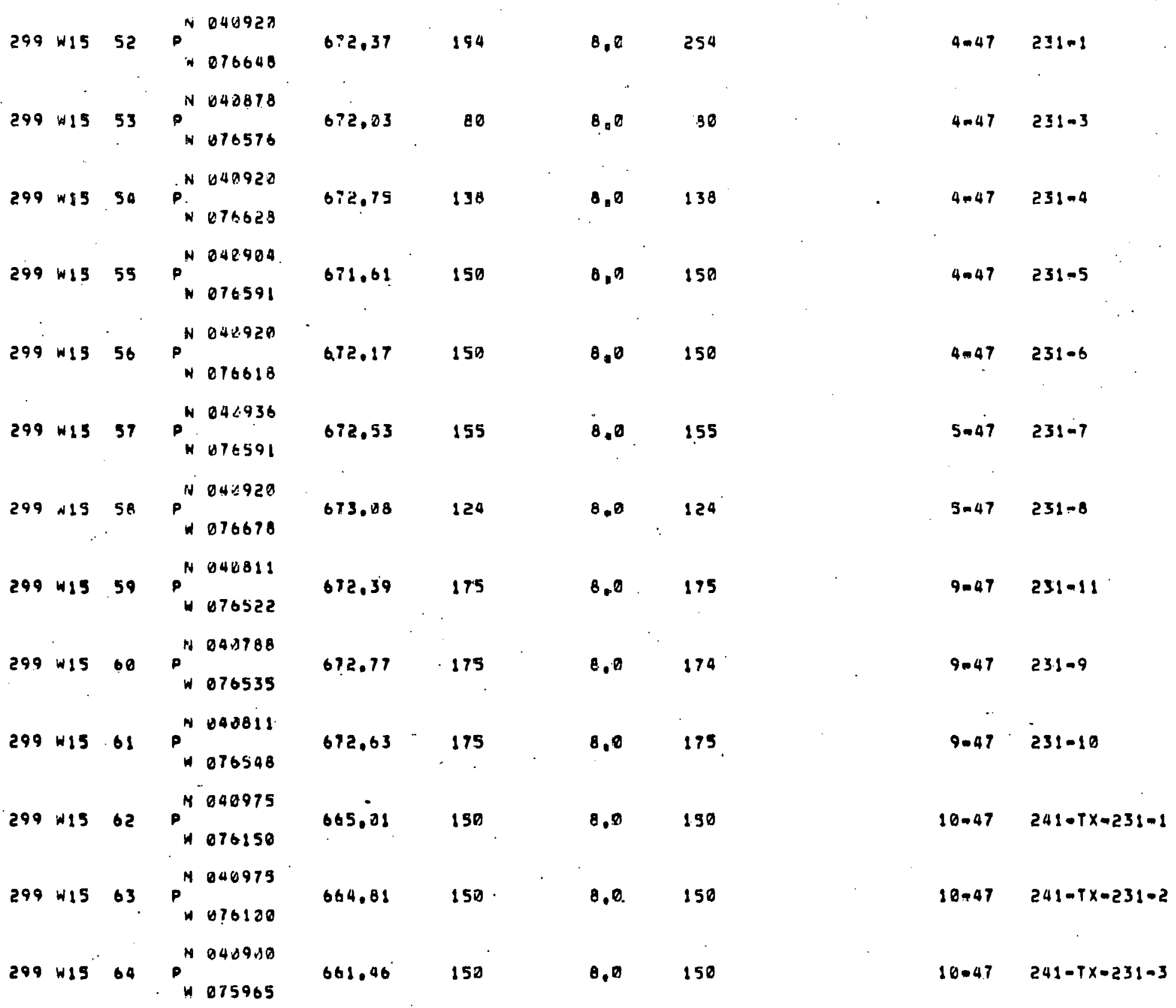




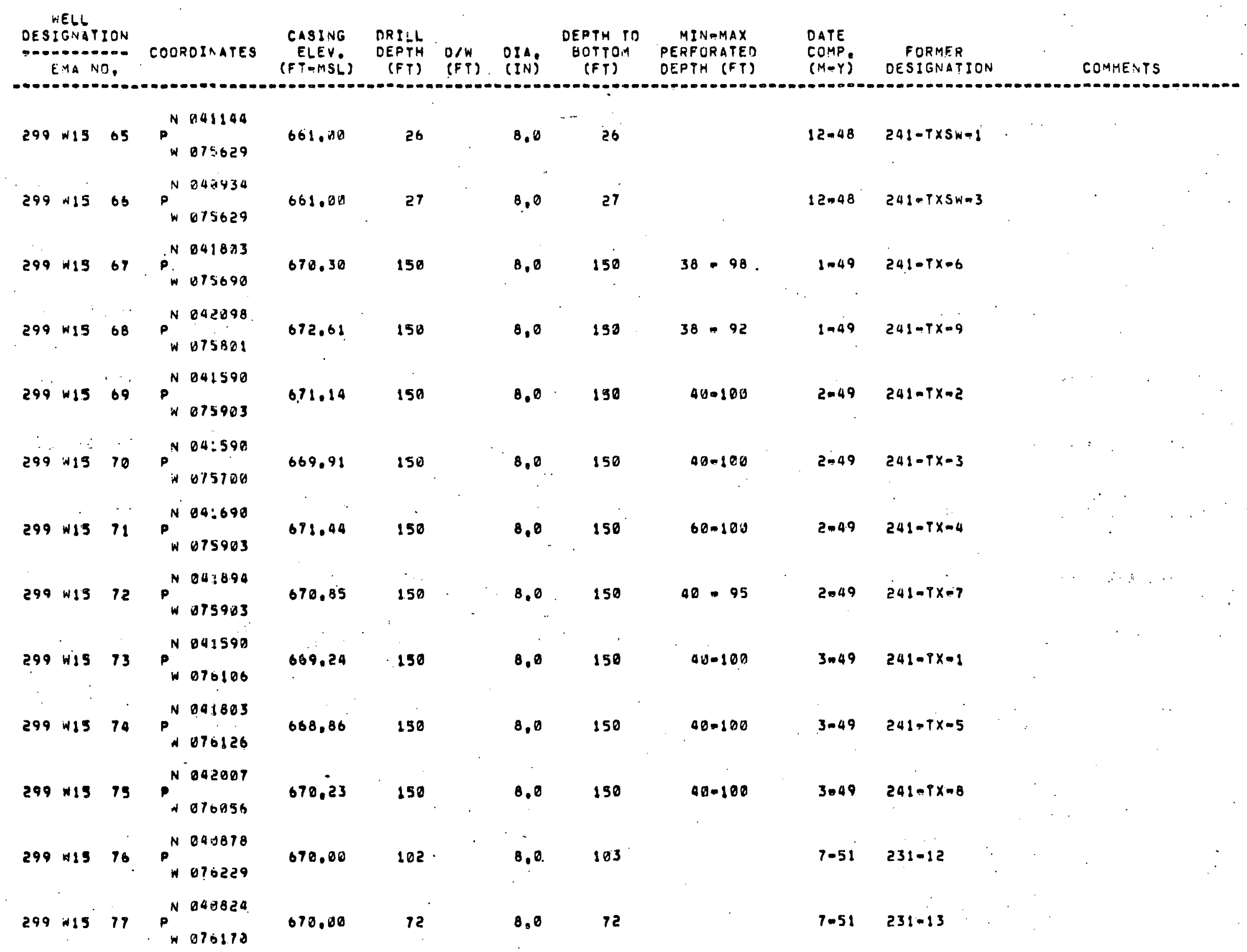


WELL

OESIGNATION

omacomana

EMA NO.

CGOROIVATES

CASING

DRILL

D/W DIA, DETTCM

MINAMAX
PEPFORATED

DATE

(FY) (FT) (IV) (FT) DEPTH (FT)

COMP.

$(M-Y)$

FORMER

DESIGNATION

COMMENTS

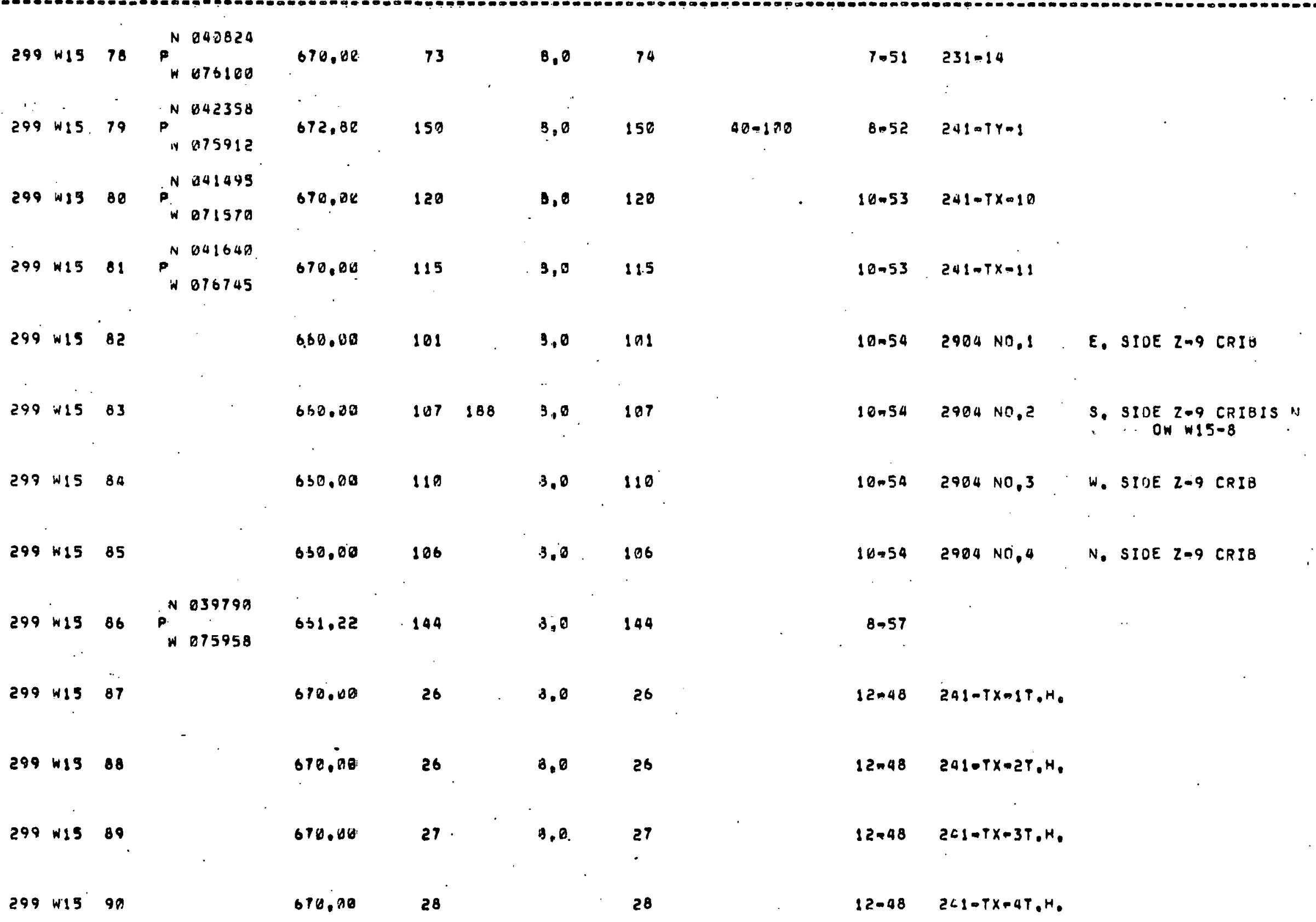


WELL

DESIGNATION

CASING DRILL

DEPTH D/W OIA DEPTH TO MINAMAX

BOTTCM PERFORATED

DATE

(FT-MSL) (FT) (FT) (IN)

(M-Y) DESIGNATION

COMMENTS

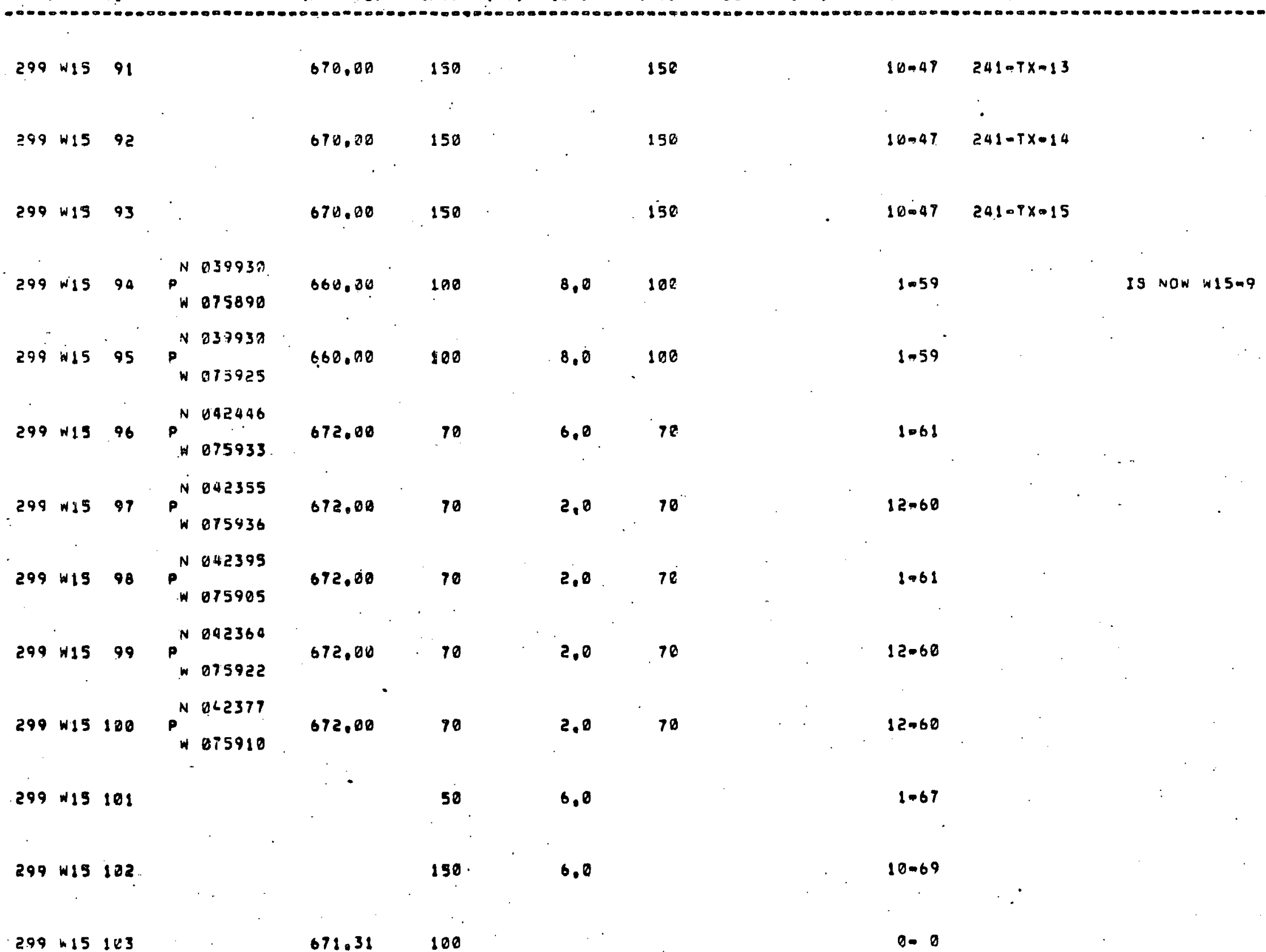


WELL

OESIGNATION

COJRUINATES

CASING ORILL

OEPTH TO

EMA NO. (F:-MSL)

$299 \times 15 \quad 100$

$671.17 \quad 100$

$0 \cdot 0$

$299 \times 15 \quad 105$

670.59

299415126

690.20

D. 0

299 wis 107

670.21

100

Q.

$299 \quad W 15 \quad 168$

660.66

100

0. 0

299 W19 109

667.35

200

$0=0$

$299 \times 15 \quad 128$

668.60

100

$a-0$

$299 \times 15111$

667.95

190

Q. 0

$299415: 12$

$670.12 \cdot 100$

on 0

$299 \quad 415 \quad 113$

$571.06 \quad 100$

D- 0

299 W15 110

$671.14 \quad 180$

D. 0

299 W13.115

670.75

100

0

$299+15116$

$869.90 \quad 100$

D. 0 


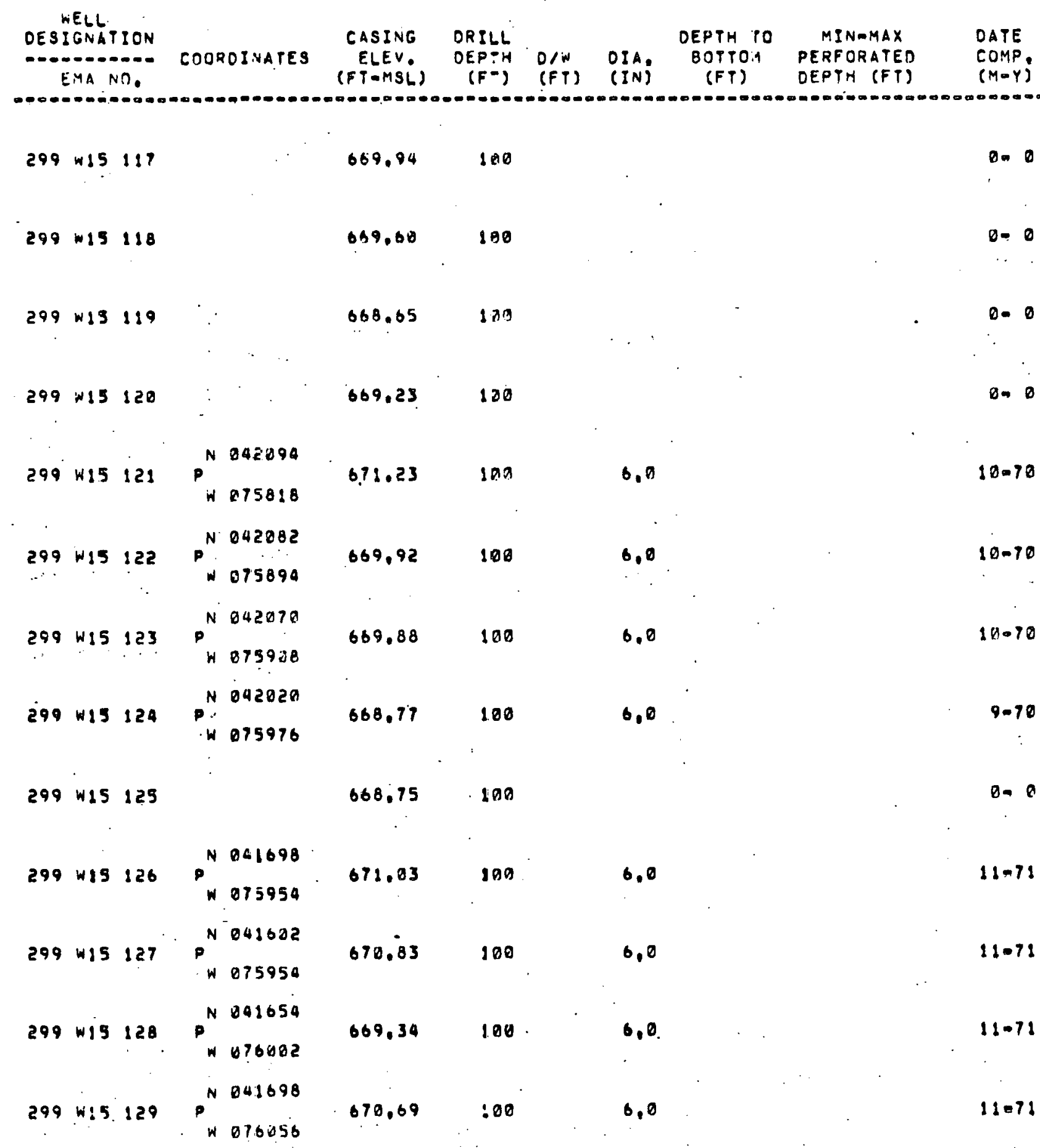




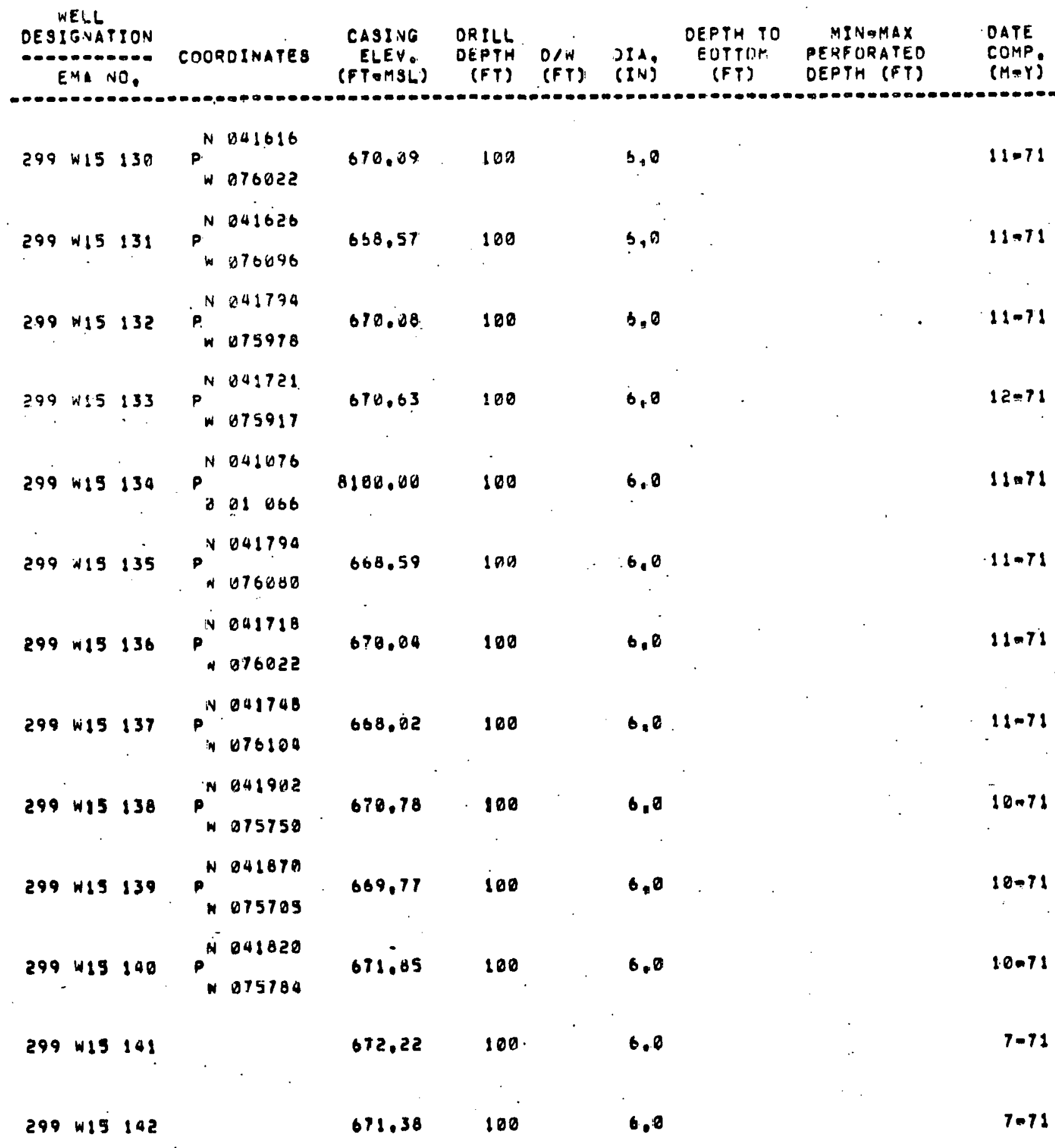


WELL

DESIGNATION

-omencueno.

. EMA NO.

COOROINATES

CASING

ELEY ORILL $(F T \rightarrow M S L)$

DEPYH

D/W DIA DEPTH PO

DEPTH 10 MINAMAX

(FT)

PERFORATEO

OEPTH (FT)

DATE

COMP. FORMER

(M-Y) OESIGNATION

COMMENTS

$299 \times 15 \quad 143$

$672,24 \quad 100$

6.0

$7-71$

299 N15 144

$673.09 \quad 104$

6.0

$6+91$

$299 \times ! 5 \quad 145$

$674.06 \quad 100$

6,0

$6-71$

$299 \times 19146$

$672.45 \quad 100$

6.0

$6-71$

$299^{\circ}$ W $15 \quad 147$

671.91

100

6,0

$7-7 !$

$299 W 15148$

672,59

100

6,9

6.71

$299 W 15149$

$672.37 \quad: 00$

6.0

7.71

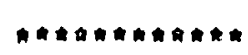

$299 \times 15155$

$672.04 .: 15$

.6 .8

$12-73$

$299 W 15156$

299 WIS 157

$299 \quad 15 \quad 158$

$299 * 15 \quad 159$

668.45

$: 00$

$11-73$

D. 0

$0=0$ 
WELL

OESIGNATION

OESIGNATION COOROINATES

EMA NO.

CASING ORILL
ELEV, DEPTH O/W DIA DEPTH TO MUTTHM PERMMAX

11073

299 W1S 162

$670.39 \quad 100$

$11-73$

$299+15 \quad 163$

671.08

100

$12-73$

$299^{\circ} \times 15 \quad 169$

670.46

100

$10-73$

$299 \times 15165$

668.68

100

b. o

$11+73$

299 WIS 166

673.05

112

b. 0

1074

$299 \quad W 15 \quad 167$

$672.32 \quad 115$

6.0

$12-73$

$299 W 15168$

672.45

180

6.0

$4 \cdot 74$

299 W15 169

672.12

120

9074

$299 \times 15 \quad 170$

672.45

111

6,0

197.4 


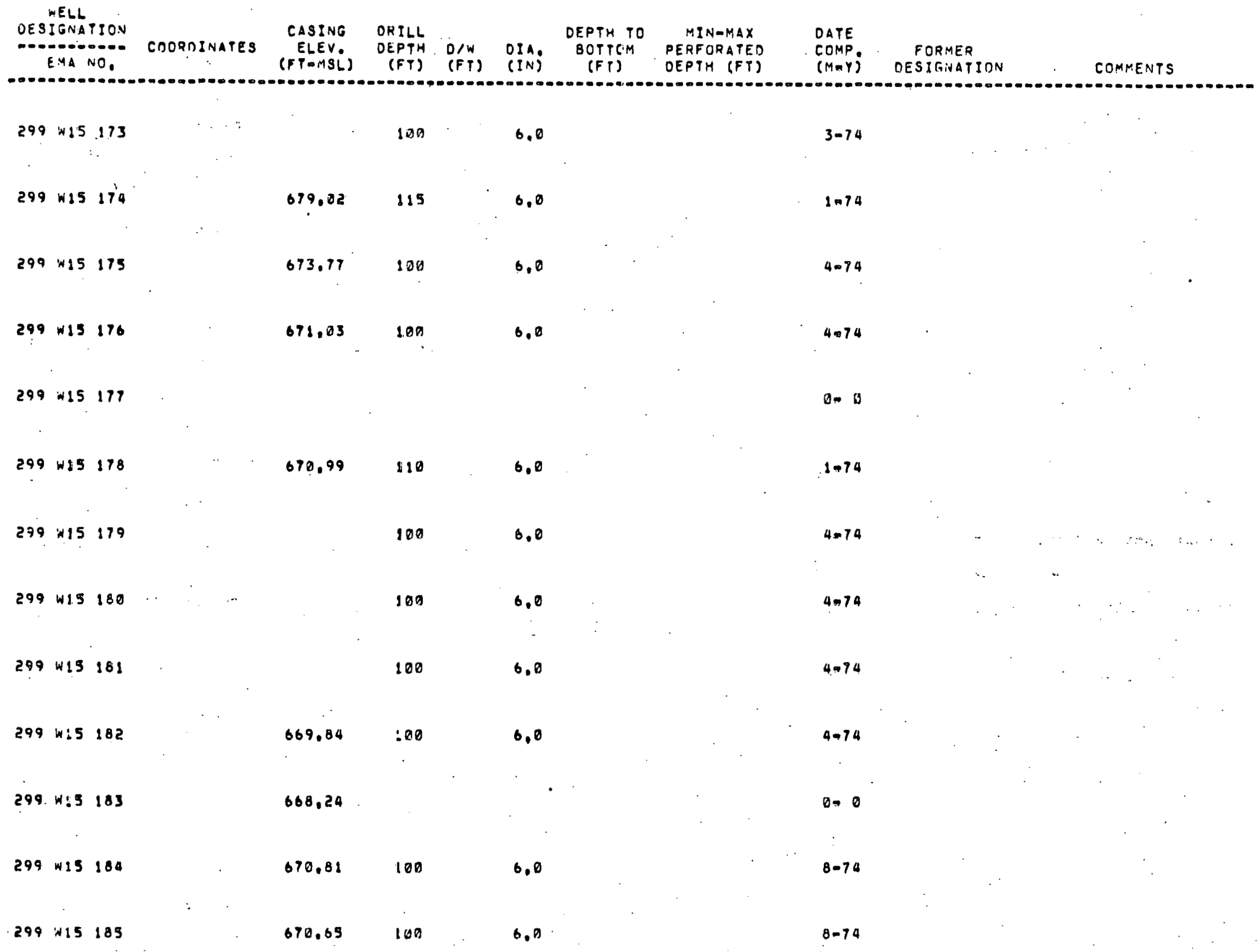


WELL

DESIGNATION

COOROINATES

CASING ORI

EYA NO

FY-MSi

O/W OIA DEPTH TO

MIN-MAX

$\begin{array}{ll}\text { BOTTISM } & \text { PERFORATED } \\ \text { (FT) } & \text { DEPTH (FT) }\end{array}$

DATE

COMP

GORMER

DESIGNATION

299 W15 186

671.05 .100

6.0

8.74

$299 \times 18: 1 P^{N 935}+039388$

$68 \pi .00$

$4.27 \quad 208$

8.0

$195-425$

$1=59$

$290 \times 182 P^{N 039120}$

$681.40 \quad 280 \quad 208 \quad 0.0 \quad 246 \quad 280.278 \quad 11.58$

6 IN. SCREEN 205-255

$299 \operatorname{lig}_{2936} \quad F^{N 039600}$

688.02

$450^{\circ} \quad 215$

$6.024 ?$

$205-447$

$1=59$

6'IN. SCREEN 20P-25S

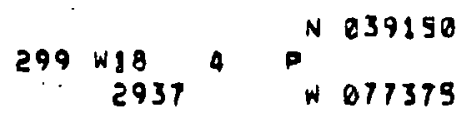

684.08

$230 \quad 211$

$8,0 \quad 246 \quad 200-278$

$2=59$

- IN. SCREEN 194-254

299 W18 $5 P^{N 039350}$

683.00

280211

272

195-274 $\quad 11.58$

$\begin{array}{lll}299 & 18 & P^{N} 039224 \\ 2951 & N 076491\end{array}$

$299 \times 18, p^{Y 039212}$ 2952 N 076706

$299 \times 18$
$297 !$

$299 \begin{array}{ccc}H 8 & 9 & P^{M} 038852 \\ 2964 & H 076846\end{array}$

682.47

350204

6,0

$1900-298$

1064

$300203 \quad 8.0$

$1900298 \quad 1064$

$212 \quad 202 \quad 6.0$

NONE

1067

$299 \mathrm{HIS.19} P^{N 038847}$ $2965 * 076003$

682.63

$220 \quad 208$

6.0297

$180-218$

12068

299 W18 il $p^{\text {No38735 }}$ 2966 W $\$ 2955$

$22208 \quad 6.0$

1900229

$\theta=0$

299 W18 $12 p^{N} 838850$

$220 \quad 208 \quad 6.0$

$190-218$

0. 0 


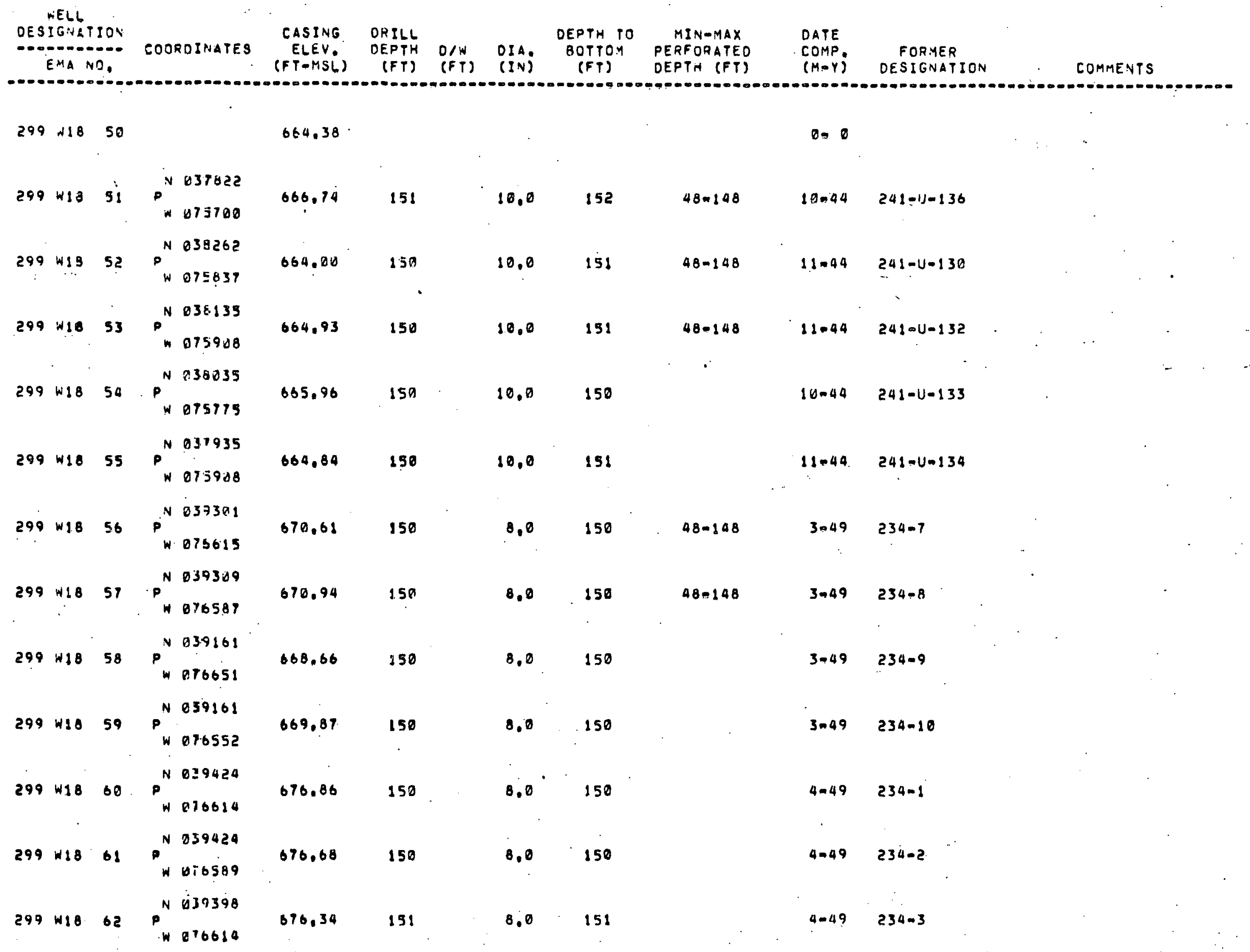




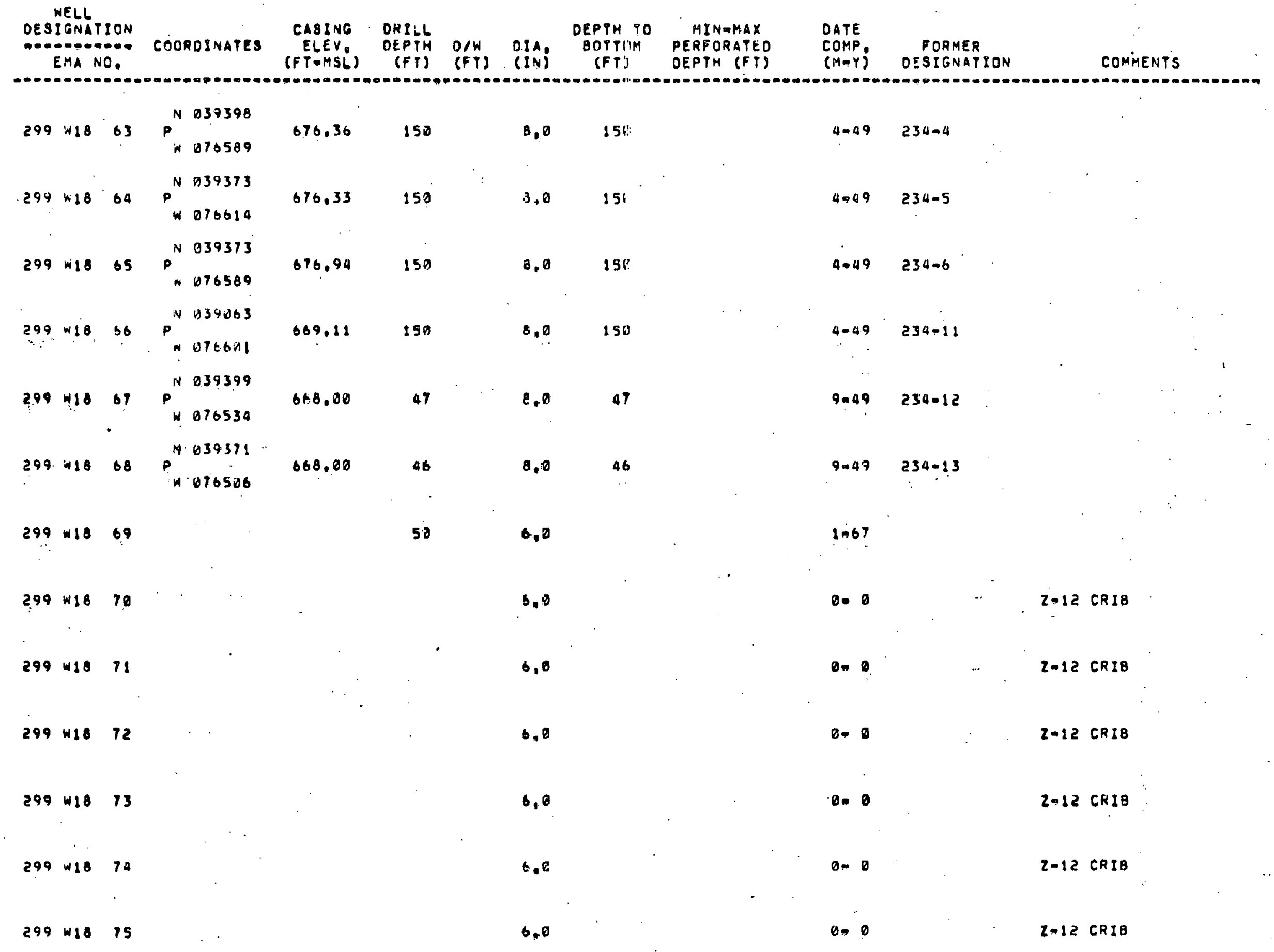




\begin{tabular}{|c|c|c|c|c|c|c|c|c|c|c|c|c|c|c|c|}
\hline OES & $\begin{array}{l}\text { WELL } \\
\text { IGNAT } \\
\text { EMA }\end{array}$ & $\begin{array}{l}10 N \\
0.0\end{array}$ & COOROINATES & $\begin{array}{l}\text { CASING } \\
\text { ELEV. } \\
\text { (FT-MSL) }\end{array}$ & $\begin{array}{l}\text { DRILL } \\
\text { DEPTH } \\
\text { (FT) }\end{array}$ & $\begin{array}{l}0 / W \\
\text { (FT) }\end{array}$ & $\begin{array}{l}\text { OIA; } \\
\text { (IN) }\end{array}$ & $\begin{array}{l}\text { DEPTH TD } \\
\text { BOTYO } \\
\text { (FT) }\end{array}$ & $\begin{array}{l}\text { MIN-MAX } \\
\text { PERFORATEO } \\
\text { OEPTH (FT) }\end{array}$ & $\begin{array}{l}\text { DATE } \\
\text { COMP } \\
\text { (MOY) }\end{array}$ & $\begin{array}{l}\text { FORMER } \\
\text { DESIGNATION }\end{array}$ & & & MENTS & \\
\hline 299 & 118 & 76 & & & & & 6.0 & & & 0.0 & & $z-1$ & CRIB & & \\
\hline 299 & WI 8 & 77 & . & & & & 6.0 & & & $\theta .0$ & & $2-1$ & CRIB & & \\
\hline 259 & W18 & 78 & & . & & & 6.0 & & & 0.0 & & $z-1$ & CRIS & & \\
\hline 299 & WIO & 79 & & . & & & 6.0 & & & $0-\theta$ & & $2-2$ & CRIB & . & \\
\hline 299 & W18 & 89 & $\cdot$ & & . & & 6.0 & & . & $\theta=0$ & & $2-1$ & CRIB & & \\
\hline 299. & +1.8 & 81 & . & & & & 6,0 & & & $\theta 0$ & & $2-10$ & CRIB & & \\
\hline 299 & $w: 8$ & 82 & . & & & & & & & $0=0$ & & 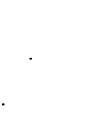 & & & \\
\hline 299 & W18 & 83 & & & & & & & & $0=0$ & & & & & \\
\hline 299 & $W 18$ & 84 & & & 190 & & 6.0 & & $\cdot$ & $7=69$ & & $\cdot$ & & & \\
\hline 299 & $w 18$ & 85 & $\begin{array}{l}P_{W}^{N} 038989 \\
W 0717\end{array}$ & 679.75 & 150 & . & 6,0 & 150 & & 7969 & & & & - & \\
\hline 299 & 118 & 86 & $\begin{array}{l}P^{N} 039100 \\
W 076742\end{array}$ & 683.49 & 150 & & 6.0 & 150 & & 8.69 & & & & & \\
\hline 299 & wis & 87 & $\begin{array}{l}P^{N 038980} \\
W 070004\end{array}$ & 677.23 & 150 & & 6.9 & 150 & & $9-69$ & .. & & & & \\
\hline 299 & $W 18$ & 88 & $\begin{array}{l}P^{N} 039293 \\
W 076432\end{array}$ & 679.76 & 150 & $\therefore$ & 6.0 & 150 & & $9-69$ & & & & & \\
\hline
\end{tabular}




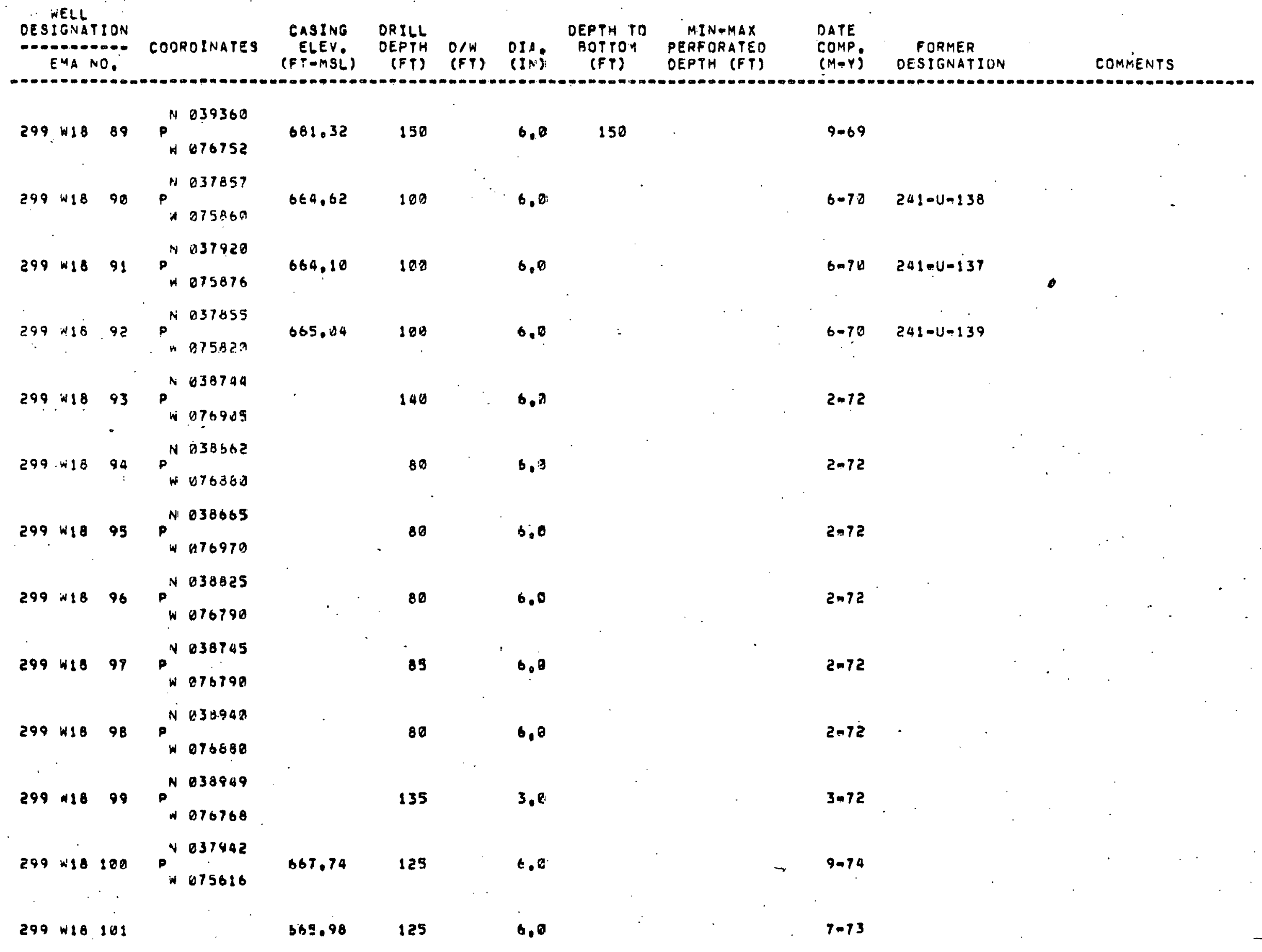


WELL

OESTGNATION

oesignarron

COUROIVATES

$\begin{array}{lll}\text { CASING ORILL } & \text { DEPTH TO MINMMAX } \\ \text { ELEV. DEFTH D/W OIA. BOTTCM PERFORATED }\end{array}$

DEPTH (FT)

DATE

E4) NO,

(FP) (FT) (IN) (FP)

COMP

$(M-Y)$

FORMER

DESIGNATION

COMMENTS

299 W18 102

666.29

6.0

7.73

$299 \times 18 \quad 183$

$664.94 \quad 125$

6.0

6.73

299 wis 100

$667.43 \quad 129$

0,0

6.74

299 wio 105

$669.92 \quad 125$

6,0

6073

$299 \times 18106$

On $D$

$299 \times 18 \quad 189$

$\theta=0$

299 W:8 180

0.0

299 W:8.109

665.95

$: 25$

6.0

4.74

$299 \times 48 \quad 118$

665.65

$\lcm{25}$

6.0

5.74

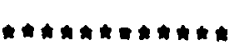

$299 W 18 \quad 112$

On. 0

$299 \times 10113$

664.63

125

6.0

$7-74$

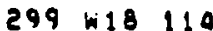

0.0 
WELL

DESIGNATION

-...-o.m-. coOrdinates EMA NO.

CASING ORILL

ORILL

D/W

DIA. DEPTH TO

MINDMAX
PERPORATED DEPTH (FT)

DATE $(F P-M S L)$

(FI) (FT) (IN) / (FT)

COMP.

$299 W 18 \quad 115$

$606.10 \quad 125$

0,0

$3-74$

$299 \times 18 \quad 116$

$299 \times 10 \quad 117$

666.47

125

6,0

5.74

299 WIB 118

$299 \times 18119$

664.64

125

0.0

5.79

299 W18 120

$299 \times 18121$

$299 \times 18.122$

664.25

120

6. 2

$2-74$

$299 \times 18 \quad 123$

663.84

125

6.0

$7+74$

$299+18 \quad 124$

668.83

120

3. 0

2.74

299 W18 125

668.76

820

0,0

$2-74$

$299 W 18 \quad 126$

660.93

125.

6,0

3.74

$299 \times 15129$

655.63

125

6.0

$5-74$ 

WELL

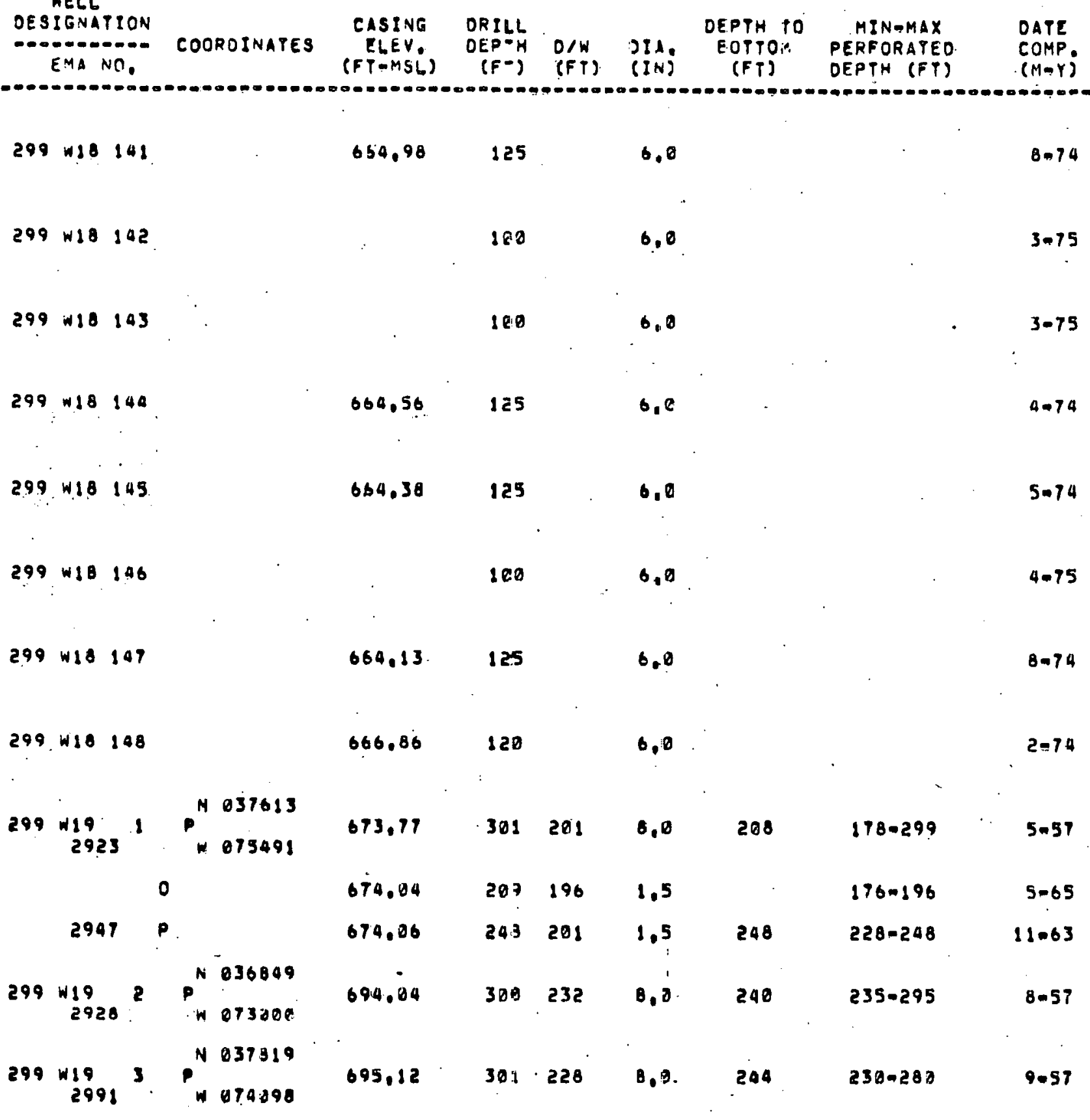




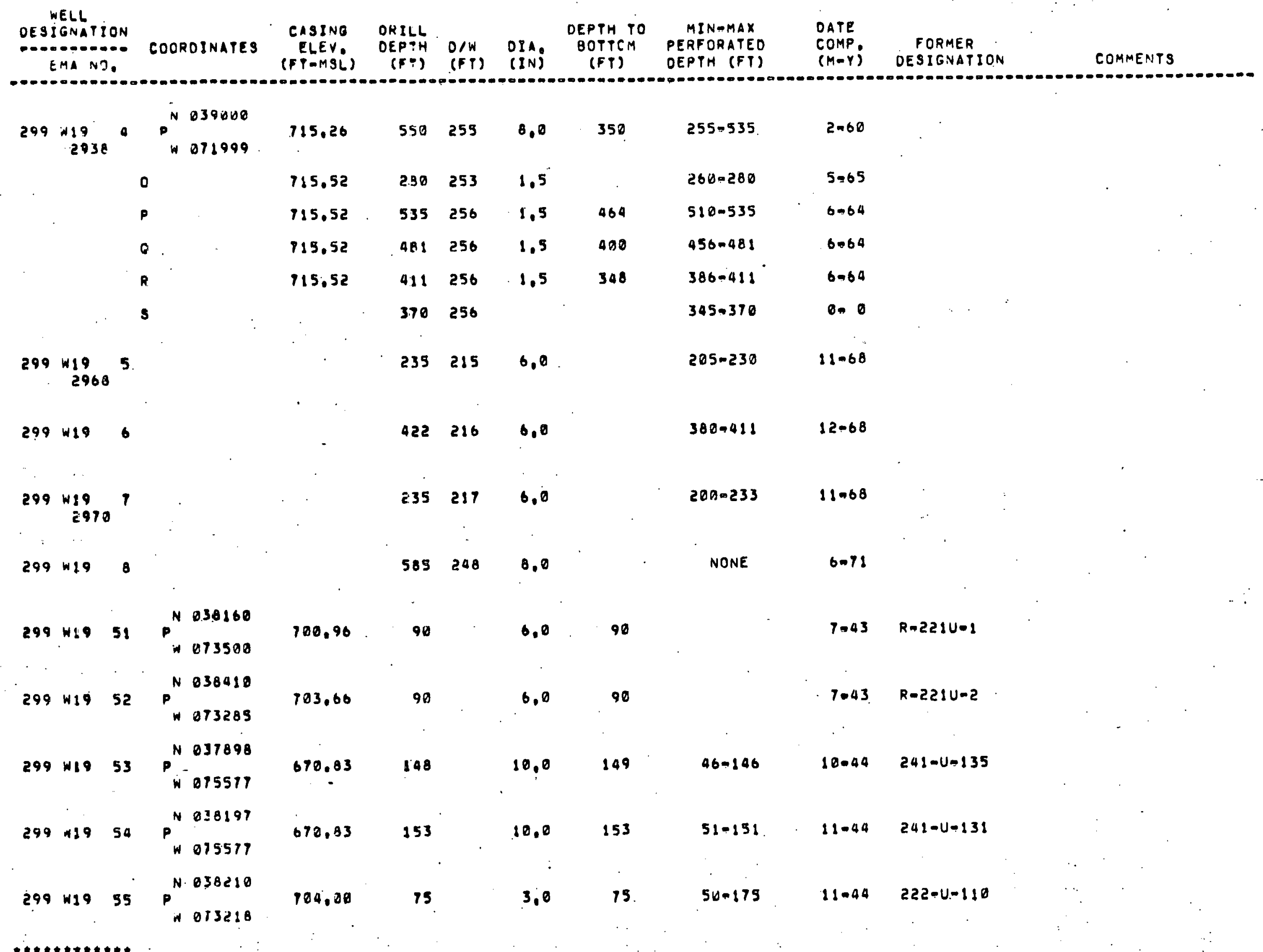




\begin{tabular}{|c|c|c|c|c|c|c|c|c|c|c|c|}
\hline $\begin{array}{r}W \\
\text { DESI } \\
-O\end{array}$ & $\begin{array}{l}\text { NELL } \\
\text { IGNATIS } \\
\text { EA NO }\end{array}$ & & CODQOINATES & $\begin{array}{l}\text { CASING } \\
\text { ELEV } \\
(F T-M S L)\end{array}$ & $\begin{array}{l}\text { DRILL } \\
\text { DEPTH } \\
\text { (FT) }\end{array}$ & $\begin{array}{l}D / W \\
(F Y)\end{array}$ & $\begin{array}{l}\text { OIA: } \\
\text { IIN) }\end{array}$ & $\begin{array}{l}\text { DEPTH TO } \\
\text { BUPTIM } \\
\text { FFT. }\end{array}$ & $\begin{array}{c}\text { AINAMAX } \\
\text { PERFURATED } \\
\text { OEPTH (FT) }\end{array}$ & $\begin{array}{l}\text { DATE } \\
\text { COMP } \\
(M-Y)\end{array}$ & $\begin{array}{l}\text { FORMER } \\
\text { OESIGNAT }\end{array}$ \\
\hline 299 & was & 69 & $\begin{array}{l}P_{W 073100}^{N 036920} \\
W 07\end{array}$ & 692.00. & 49 & - & 8.0 & 44 & & 1.51 & $216-W R=1$ \\
\hline 299 & $w 197$ & $\begin{array}{r}79 \\
:\end{array}$ & $\begin{array}{l}F^{N .036860} \\
W 073100\end{array}$ & 692,08 & 105 & & 8.0 & $10 ! 5$ & & $1-5 !$ & $216-W R-2$ \\
\hline 299 & W19? & 71 & $\begin{array}{l}P^{N} 0368200 \\
W 013100\end{array}$ & $692,0 \mathrm{E}$ & 117 & & 8,0 & 117 & & $\mid-51$ & $216-W R=3$ \\
\hline 299 & $\begin{array}{lll} & 19\end{array}$ & 12 & $\begin{array}{l}\text { N } 037859 \\
\text { N } 474247\end{array}$ & 925.60 & 50 & & 8,0 & $5 n$ & & $2-5 !$ & $216-U-1$ \\
\hline 299 & $\begin{array}{l}W 19 \quad \\
\therefore\end{array}$ & 73 & $\begin{array}{l}P^{N} 037859 \\
W 070322\end{array}$ & 705.30 & 50 & & 8.0 & 50 & & 3.51 & $2: 6-U-2$ \\
\hline 299 & $\$ 197$ & 74 & & 668.45 & 125 & & 6.0 & . & & 8.74 & . \\
\hline 299 & $\begin{array}{c}\text { w19? } \\
:\end{array}$ & 76 & & 668.89 & 125 & & 6,0 & & & $B=74$ & \\
\hline 299 & $\begin{array}{l}\text { W2! } \\
2930\end{array}$ & 1 & $\begin{array}{l}P^{N} 035808 \\
* 071382\end{array}$ & 699.26 & 352 & 241 & 8,0 & 253 & $220-290$ & $9=57$ & \\
\hline 299 & W21 & 83 & & & 100 & & . & & & $4=74$ & \\
\hline 299. & W21 8 & 89 & & & 34 & & & & & $5-74$ & \\
\hline 299 & $\begin{array}{l}W 22 \\
2919\end{array}$ & 1 & P & 670.00 & 306 & 198 & $8.0^{\circ}$ & 285 & $190-280$ & 6956 & $\begin{array}{l}241-5-9 \\
207-3-9\end{array}$ \\
\hline 299 & $\begin{array}{l}\text { N22 } \\
2920\end{array}$ & 2 & Pr A1522a & 670.00 & 367 & 201 & 8.7 & 289 & $195-285$ & $5-56$ & $\begin{array}{l}241-5-10 \\
20.7-5-10\end{array}$ \\
\hline
\end{tabular}




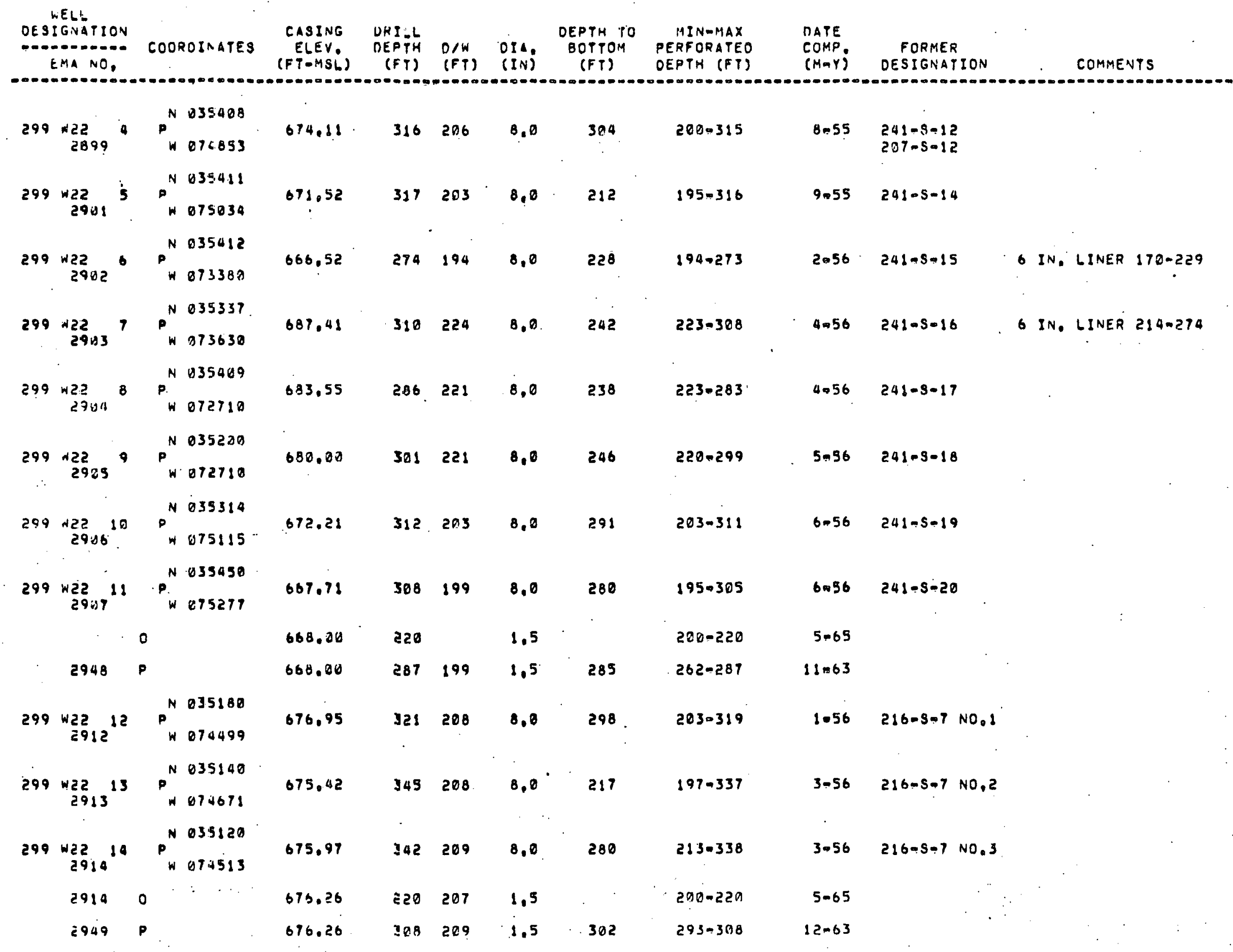




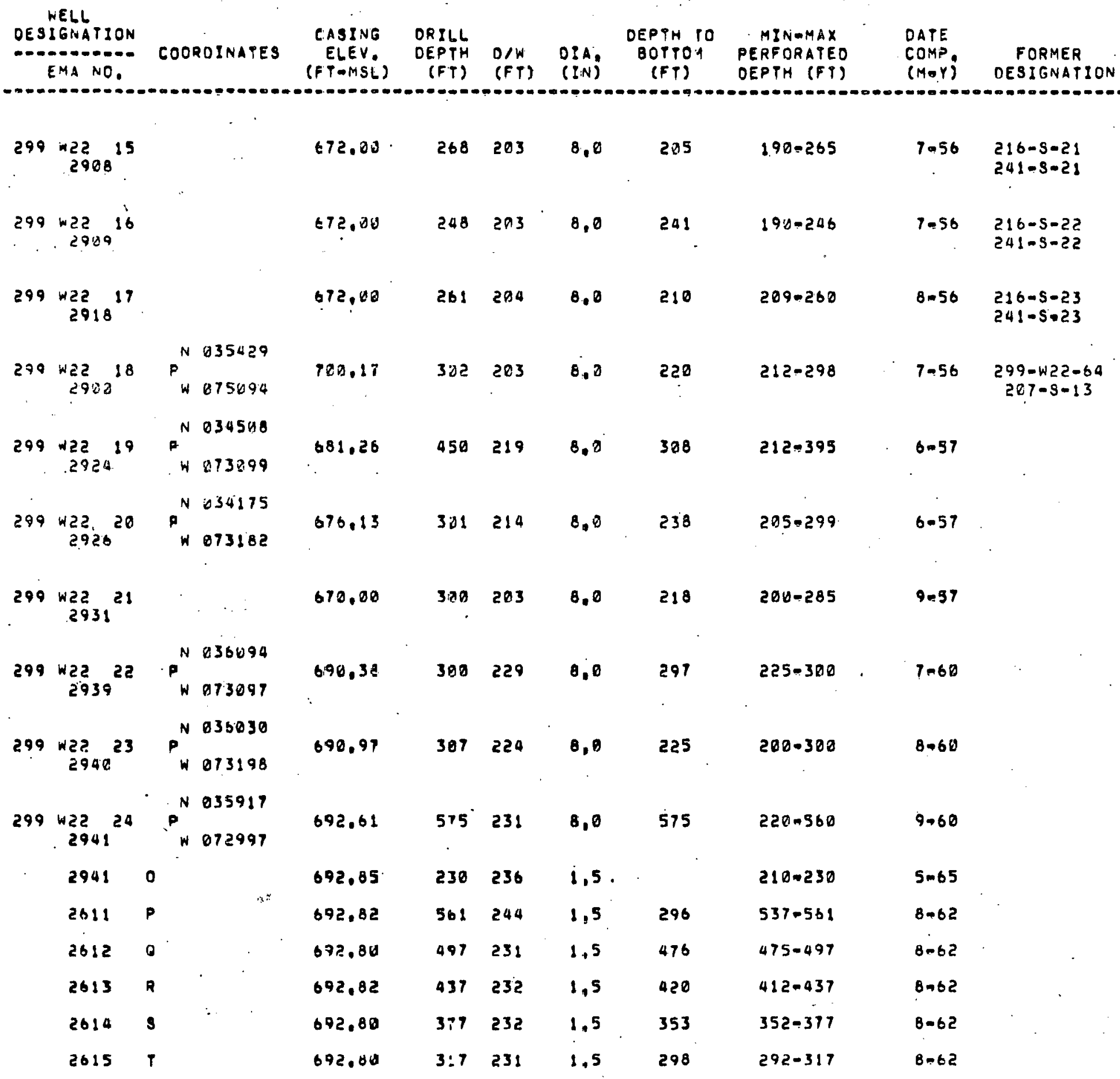




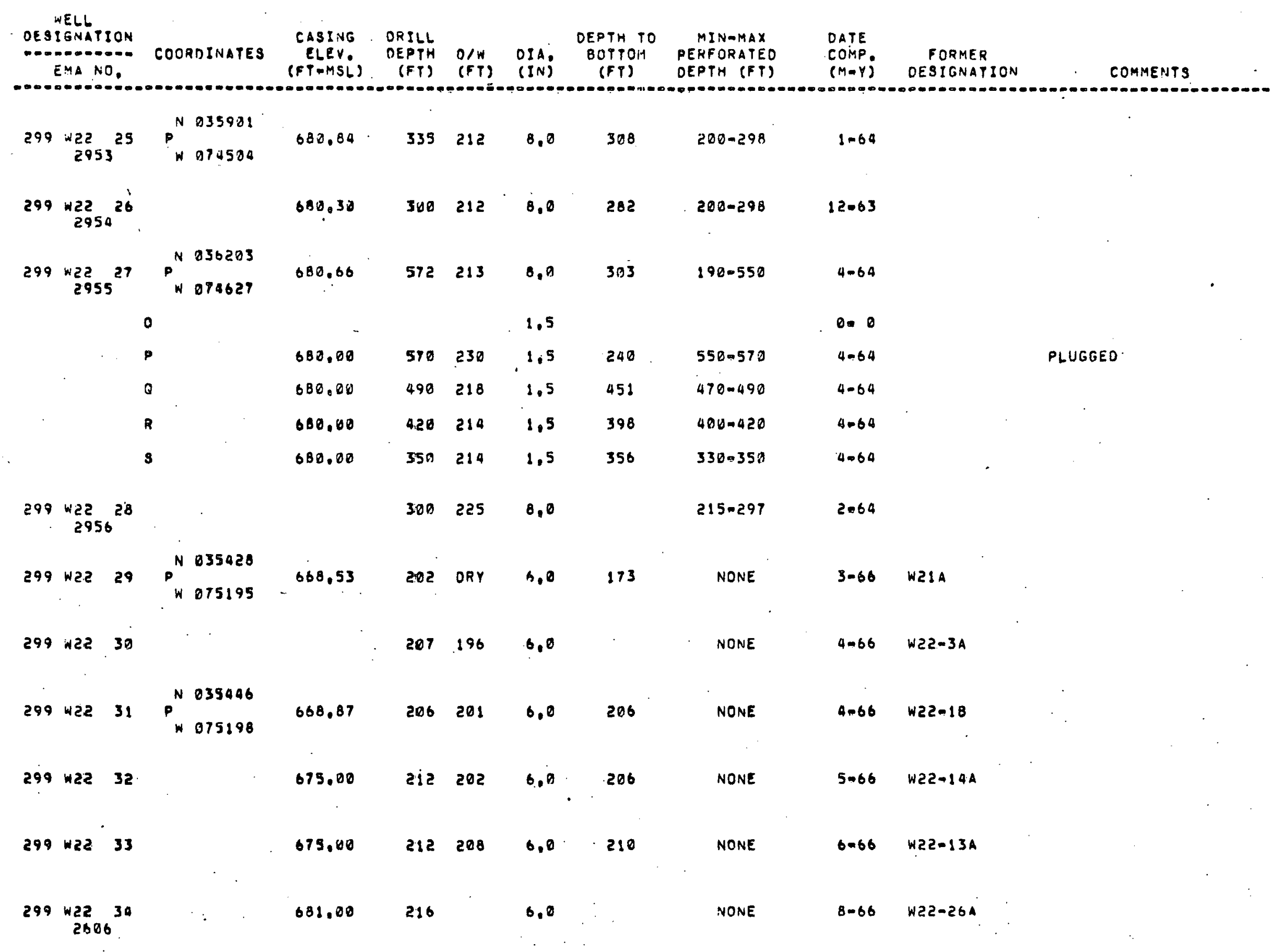




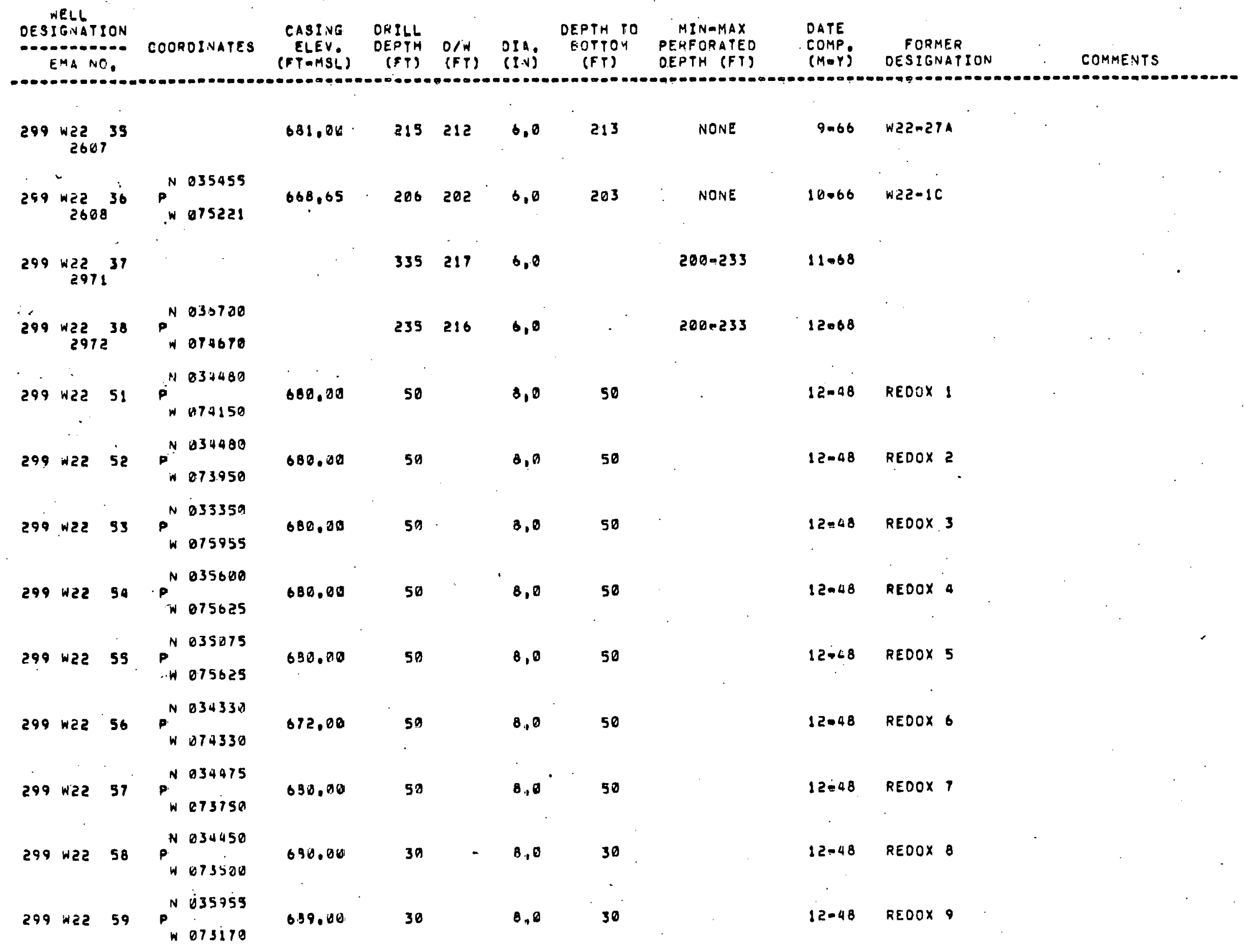




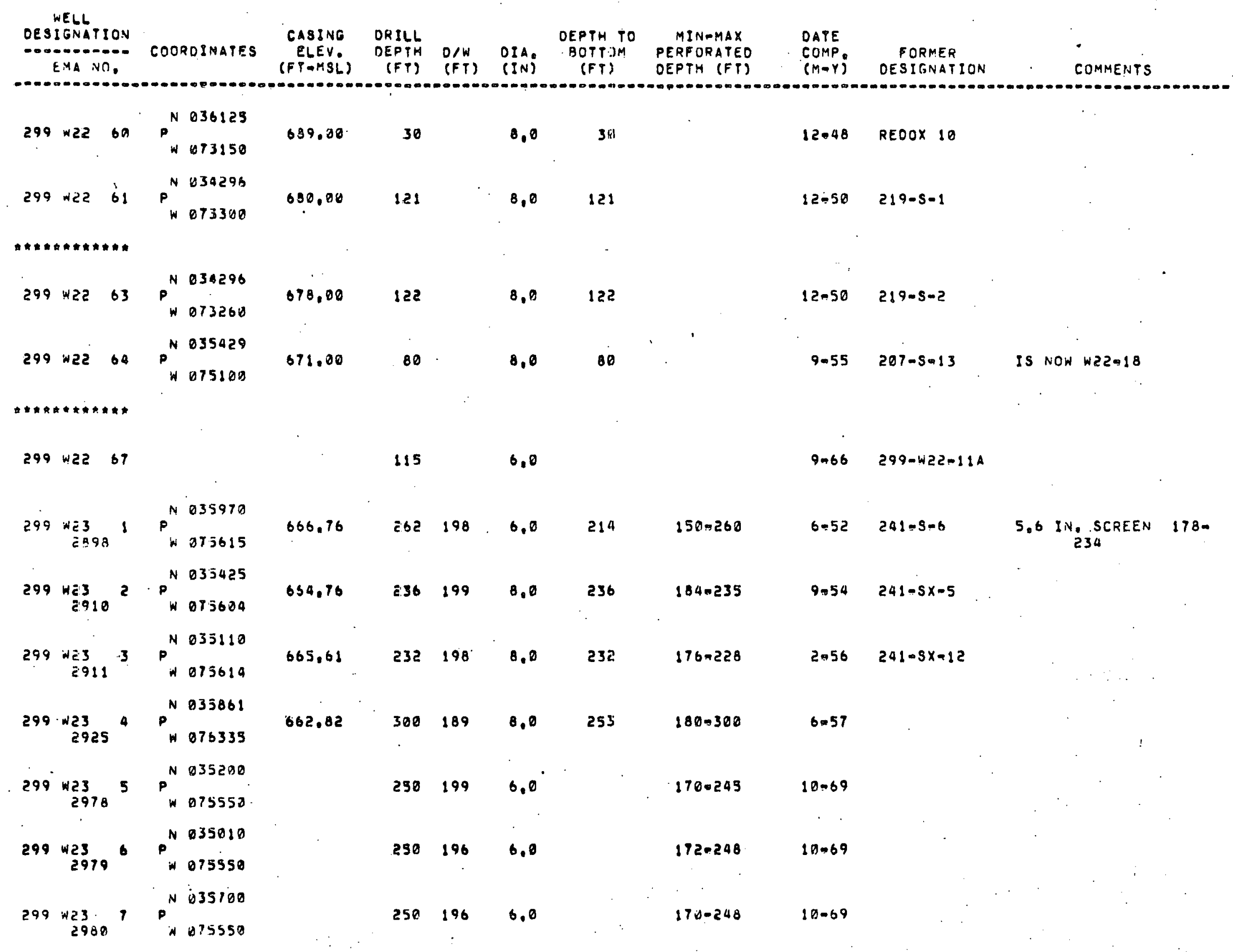




\begin{tabular}{|c|c|c|c|c|c|c|c|c|c|c|c|c|c|c|}
\hline $\begin{array}{r}\text { OES } \\
=-\infty\end{array}$ & $\begin{array}{l}\text { WELL } \\
\text { IGNATI } \\
\text { EMA NO }\end{array}$ & ON & COOR & TOINATES & $\begin{array}{l}\text { CASING } \\
E L E V . \\
(F T-M S L)\end{array}$ & $\begin{array}{c}\text { ORILL } \\
\text { DEP }=H \\
\left(F^{-}\right)\end{array}$ & $\begin{array}{l}D / W \\
(F T)\end{array}$ & DIA: & $\begin{array}{l}\text { DEPTH TO } \\
\text { EOTTCM } \\
\text { (FT) }\end{array}$ & $\begin{array}{l}\text { MINGMAX } \\
\text { PERPORATED } \\
\text { DEPTH (FP) }\end{array}$ & $\begin{array}{l}\text { OATE } \\
\text { COMP } \\
(M-Y)\end{array}$ & $\begin{array}{l}\text { FORMER } \\
\text { DESIGNATION }\end{array}$ & $\mathrm{COM}$ & MMENTS \\
\hline 299 & $\begin{array}{l}-23 \\
2992\end{array}$ & 8 & $P^{N}$ & $\begin{array}{l}035520 \\
076100\end{array}$ & 663.20 & 235 & 190 & 6,0 & . & $165-238$ & $9-72$ & & $216-5-25$ & CRIB \\
\hline 299 & $\begin{array}{l}\text { We3 } \\
2993\end{array}$ & 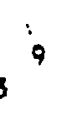 & $P^{N}$ & $\begin{array}{l}035480 \\
076320\end{array}$ & $\begin{array}{l}654.00 \\
.0\end{array}$ & 235 & 192 & 6,0 & 229 & $164-230$ & 8.72 & . & $216-5-25$ & CRI 8 \\
\hline 299 & $\begin{array}{l}W 23 \\
2994\end{array}$ & 10 & $P^{N}$ & $\begin{array}{l}035427 \\
076535\end{array}$ & 657.00 & 235 & 191 & 6,0 & 226 & 165.230 & 10.72 & & $216-5-25$ & CRIB \\
\hline 299 & $\begin{array}{l}\text { W23 } \\
2995\end{array}$ & 5 & $P^{N}$ & $\begin{array}{l}635568 \\
076725\end{array}$ & 653.06 & 255 & 189 & 6.0 & 226 & $165-230$ & 11.72 & & $216-3-25$ & CRIB \\
\hline 299 & 123 & 51 & $P^{N}$ & $\begin{array}{l}030175 \\
075615\end{array}$ & 659.00 & 150 & & 6.0 & 150 & $40-100$ & $3-52$ & $291-9+1$ & $\cdot$ & \\
\hline 299 & W? 3 & 52 & $P^{N}$ & $\begin{array}{l}036166 \\
0757 \geq 2\end{array}$ & 6.59 .20 & 159 & & 6.0 & 150 & $40=100$ & $3-52$ & $241-5-2$ & & \\
\hline 299 & w23 & 53 & $P^{N}$ & $\begin{array}{l}036186 \\
075827\end{array}$ & $669,00:$ & 150 & & 6,0 & 150 & $00-100$ & $3-52$ & $261-5-3$ & & \\
\hline 299 & W23 & 59 & $P^{N}$ & $\begin{array}{l}034082 \\
075722\end{array}$ & 669.00 & 150 & & 6,0 & 150 & $40 \cdot 100$ & $4 \cap 52$ & $261-3 \div 4$ & & \\
\hline 299 & W23 & 55 & $P^{N}$ & $\begin{array}{l}836082 \\
075827\end{array}$ & 669.00 & 190 & & 6,0 & 150 & $48-180$ & 4.52 & $241-3-5$ & & \\
\hline 299 & W23 & 56 & $P^{N}$ & $\begin{array}{l}035875 \\
075722\end{array}$ & 669.00 & 175 & & 6.0 & 150 & 40.100 & 4.52 & $24.1-5=7$ & & \\
\hline 299 & W23 & 57 & $p^{4}$ & $\begin{array}{l}035980 \\
075827\end{array}$ & 669.04 & 175 & & $6,0^{\circ}$ & 150 & $40-100$ & 4.52 & $241-5-8$ & & \\
\hline 299 & W23 & 58 & $D^{N}$ & $\begin{array}{l}035501 \\
015900\end{array}$ & 668.00 & 121 & & 8,0 & 101 & $10-99$ & 9.54 & $241-3 x-1$ & . & 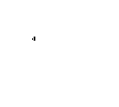 \\
\hline 299 & W23 & 59 & $p^{N}$ & $\begin{array}{l}035520 \\
015808\end{array}$ & 668.08 & 1E2 & & 8,0 & 101 & $10=99$ & $9-54$ & $241-8 x-2$ & . & \\
\hline
\end{tabular}




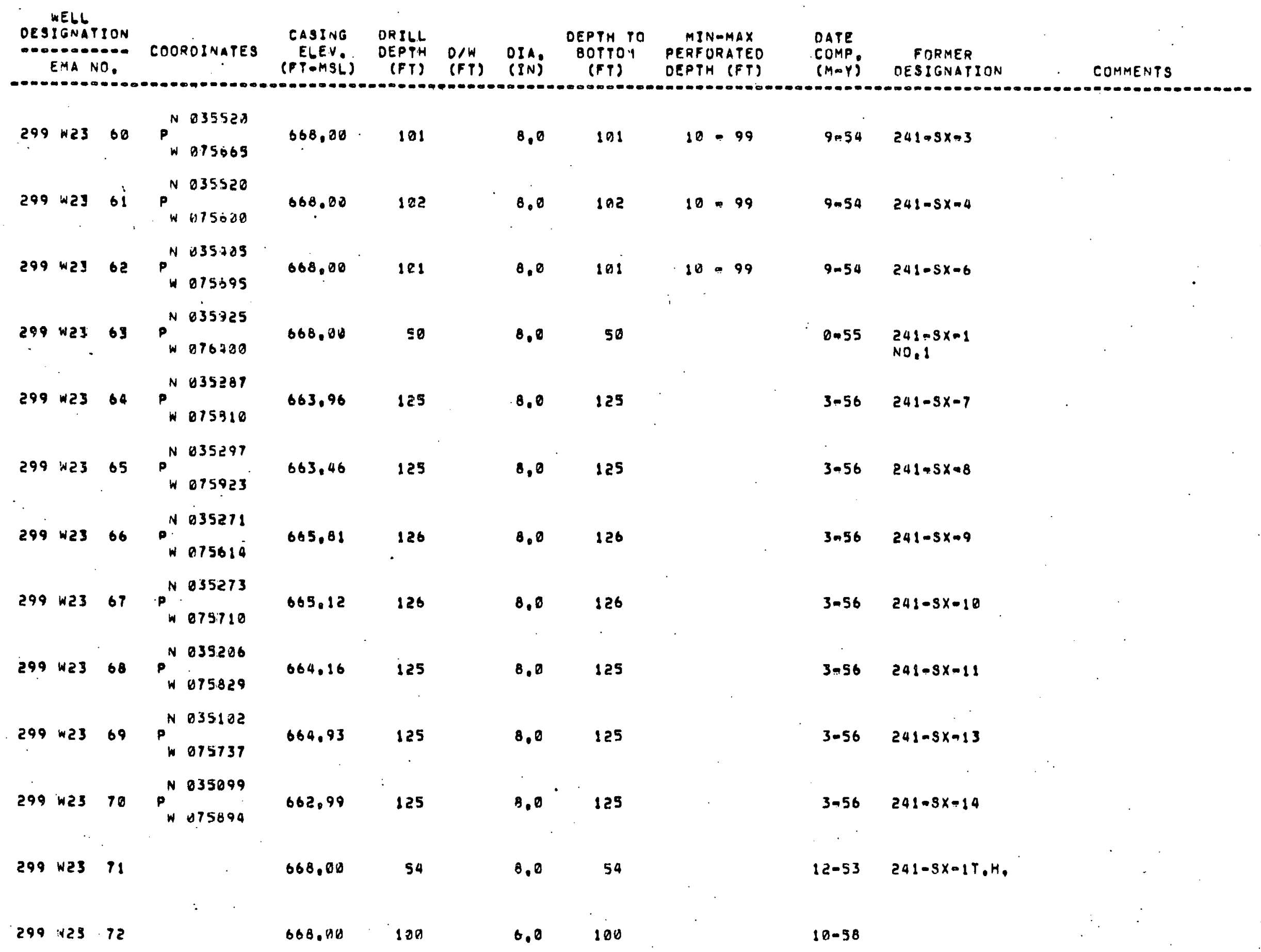




\begin{tabular}{|c|c|c|c|c|c|c|c|c|c|c|}
\hline \multicolumn{3}{|c|}{$\begin{array}{c}\text { WELL } \\
\text { OESIGGAYION } \\
\text { EMA NO. }\end{array}$} & COOROINATES & $\begin{array}{c}\text { CASING } \\
\text { ELEV. } \\
\text { (PTAMSG) }\end{array}$ & $\begin{array}{l}\text { ORILL } \\
\text { OEPTH } \\
\text { (FT) }\end{array}$ & $\begin{array}{l}0 / W \\
\text { (FT) }\end{array}$ & $\begin{array}{l}\text { DIA } \\
\text { (IN) }\end{array}$ & $\begin{array}{l}\text { DEPTH TO } \\
\text { BOTPLIM } \\
\text { GFT: }\end{array}$ & $\begin{array}{l}\text { MIN-MAX } \\
\text { PERFORAPED } \\
\text { DEPTH (FT) }\end{array}$ & $\begin{array}{l}\text { CATE } \\
\text { COMP, } \\
\text { (M-Y) }\end{array}$ \\
\hline 299 & W23 & 73 & $\begin{array}{l}P^{N} 235389 \\
W 075668\end{array}$ & 668.04 & 75 & & 8.0 & 75 & & 2062 \\
\hline 299 & $\ln 23$ & 74 & $\begin{array}{l}P^{N} 035383 \\
N 079631\end{array}$ & 668.08 & 75 & & $B, 0$ & $7:$ & & $2-62$ \\
\hline 299 & w23 & 75 & $\begin{array}{l}P^{N} 035340 \\
{ }_{W} 075687\end{array}$ & 608,08 & 75 & & $B, 0$ & 75 & & $2=62$ \\
\hline 299 & W23 & 76 & $\begin{array}{l}P^{N} 035308 \\
W 075639\end{array}$ & 668.00 & 75 & & 8,8 & 7.5 & & $2-62$ \\
\hline 299 & N23 & 77 & $\begin{array}{l}P^{N} 039302 \\
\quad 075681\end{array}$ & 668.86 & 75 & & 3,0 & 75 & & $2-62$ \\
\hline 299. & w23 & 78 & $P_{W 075711}^{N 035332}$ & 666.80 & 75 & & $B, 0$ & 75 & & $2=62$ \\
\hline 299 & W23 & 19 & $\begin{array}{l}P^{N} 235374 \\
N 375700\end{array}$ & 668.00 & 75 & & 0,0 & 75 & & $2-62$ \\
\hline 299 & W23 & BO & $\begin{array}{l}P^{v 035290} \\
\quad 075645\end{array}$ & 668.00 & 75 & & 0,0 & 135 & & $4-73$ \\
\hline 299 & W23 & $8 !$ & $\begin{array}{l}P^{N} 035262 \\
N 075617\end{array}$ & 668.00 & 75 & & 8,0 & 75 & & 2.62 \\
\hline 299 & $w 23$ & $8 z$ & $\begin{array}{l}P_{W 0352206}^{N 03538} \\
W 0756\end{array}$ & 668.00 & .79 & & $e .0$ &.$^{75}$ & & $2=62$ \\
\hline 299 & w23 & 83 & $\begin{array}{l}P^{N 035199} \\
W 075675\end{array}$ & 668.08 & 75 & & $\varepsilon, 0$ & 75 & & 2.62 \\
\hline 899 & W23 & $8 a$ & $\begin{array}{l}P_{n}^{N} 035227 \\
n 075709\end{array}$ & $B \in B, D Q$ & 15 & & 9,:D & 75 & & $2-62$ \\
\hline 299 & 123 & 85 & $\begin{array}{l}P_{H}^{N} 835287 \\
H 5690\end{array}$ & 668.00 & 75 & & 8,0 & 75 & & 3.62 \\
\hline
\end{tabular}




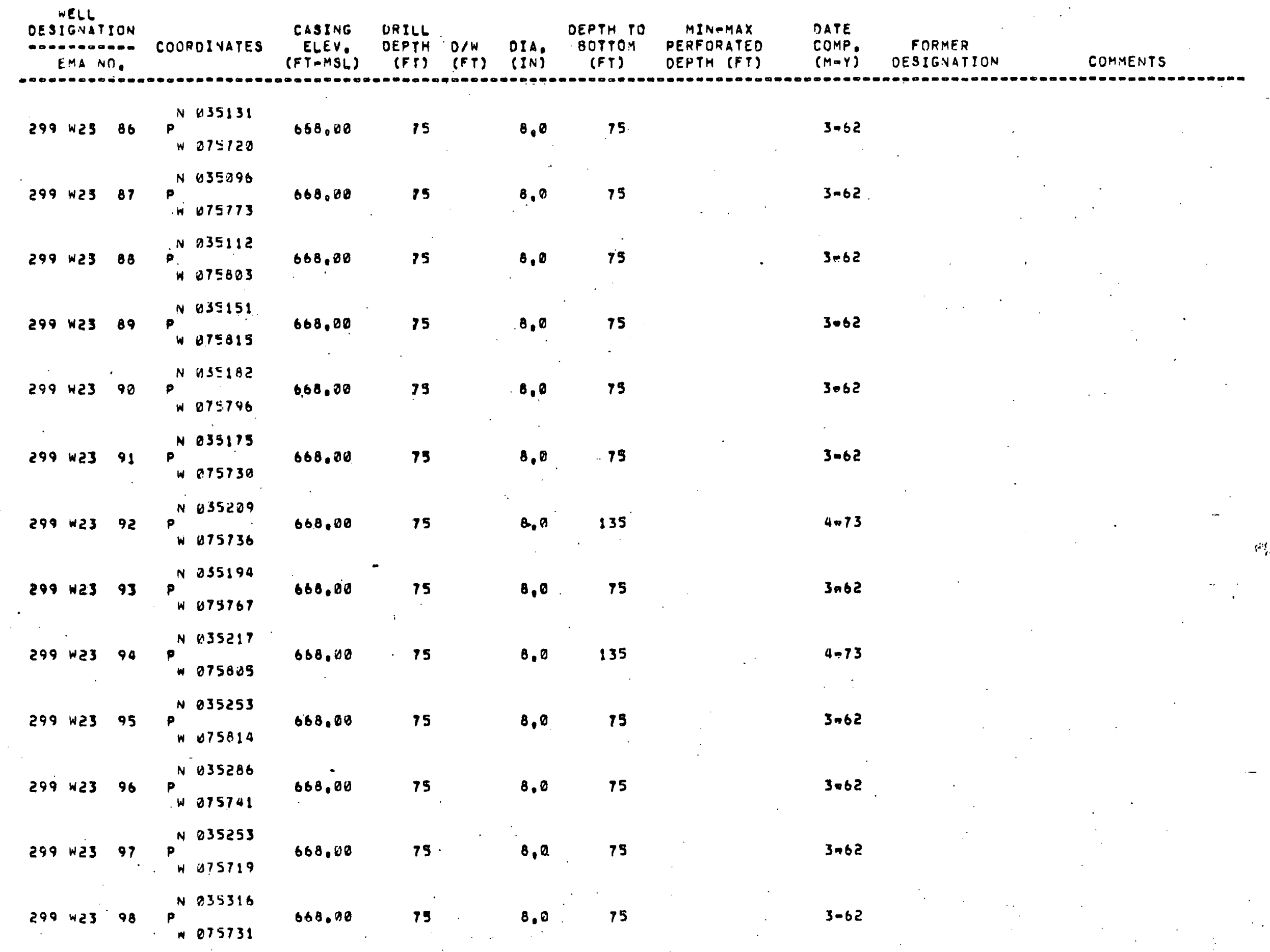


WELL

OESIGNATION

COOROINATES

CASING DRILL

MINMMAX
PEPFORATEO

OATE

Eya rio

(FTMMSL) (FP) (FP) (IV) (FP) DEPTH (FT)

(M-Y)

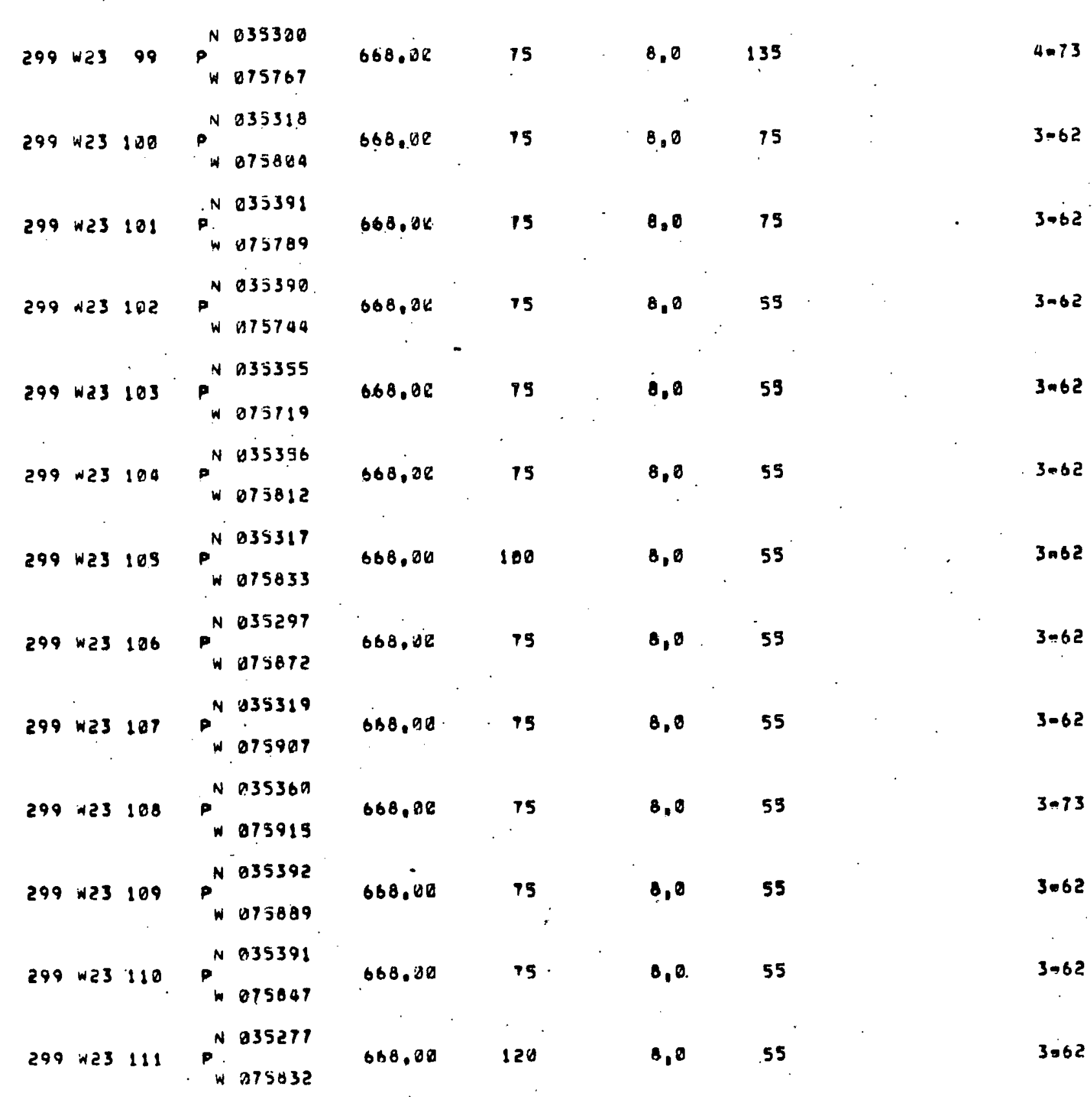


HEL:-

DESIGNATION

EMA NOR

COOROINATES

CASING ORILL

ELEY, DEPYH O/W DIA: BOTTOM PERFORATED

DATE

CUMP. FORMER

$(M-Y)$

DESIFNATION

COMMENTS

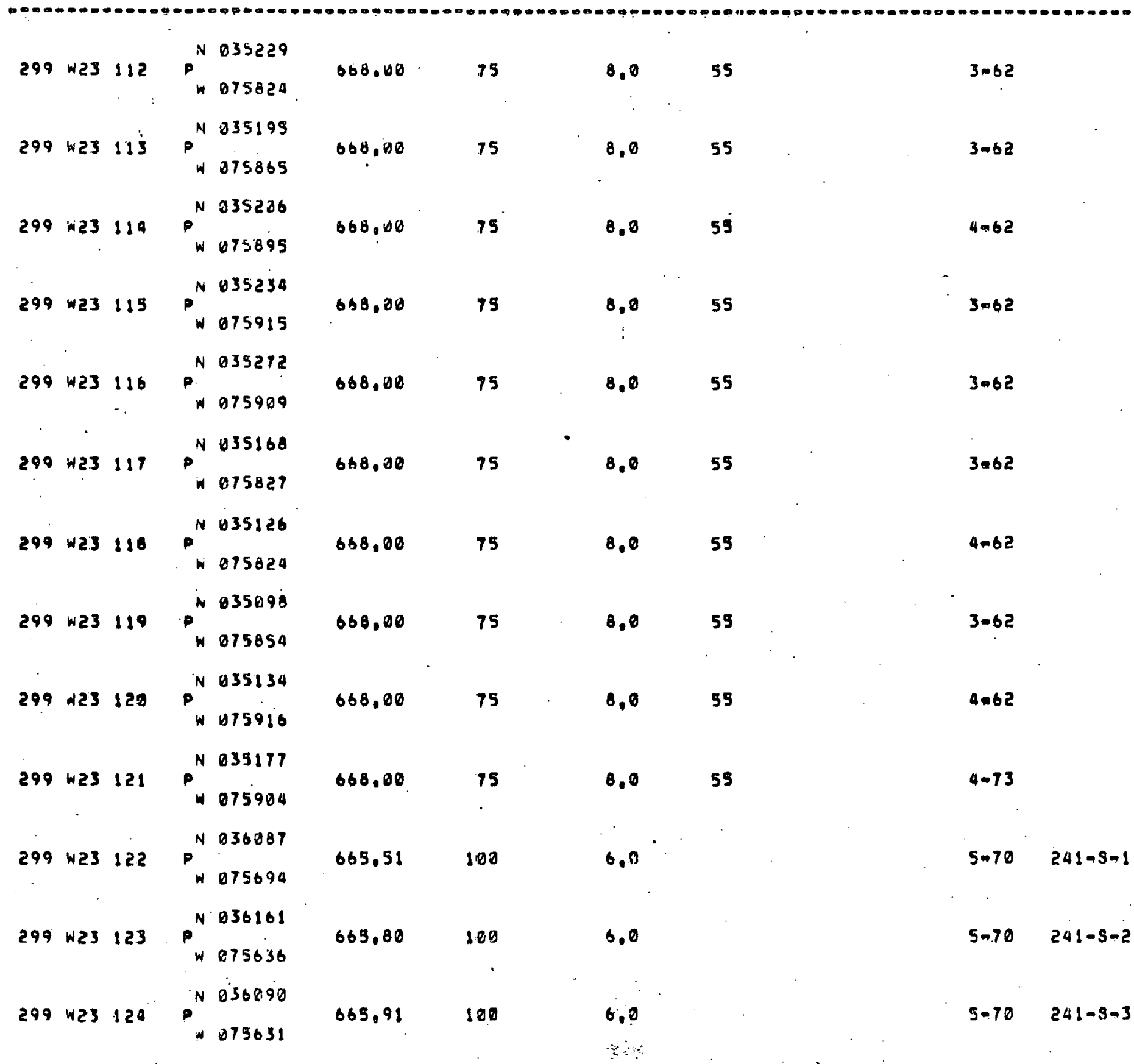


WELL

OESIGNATION

COOROINATES

EMA 10

(Fi-MSi)

299 W23 $134 P^{N} 035534$

299 W23 $135 p^{N 435590}$

$299 W 23136 p^{N 035548}$ 


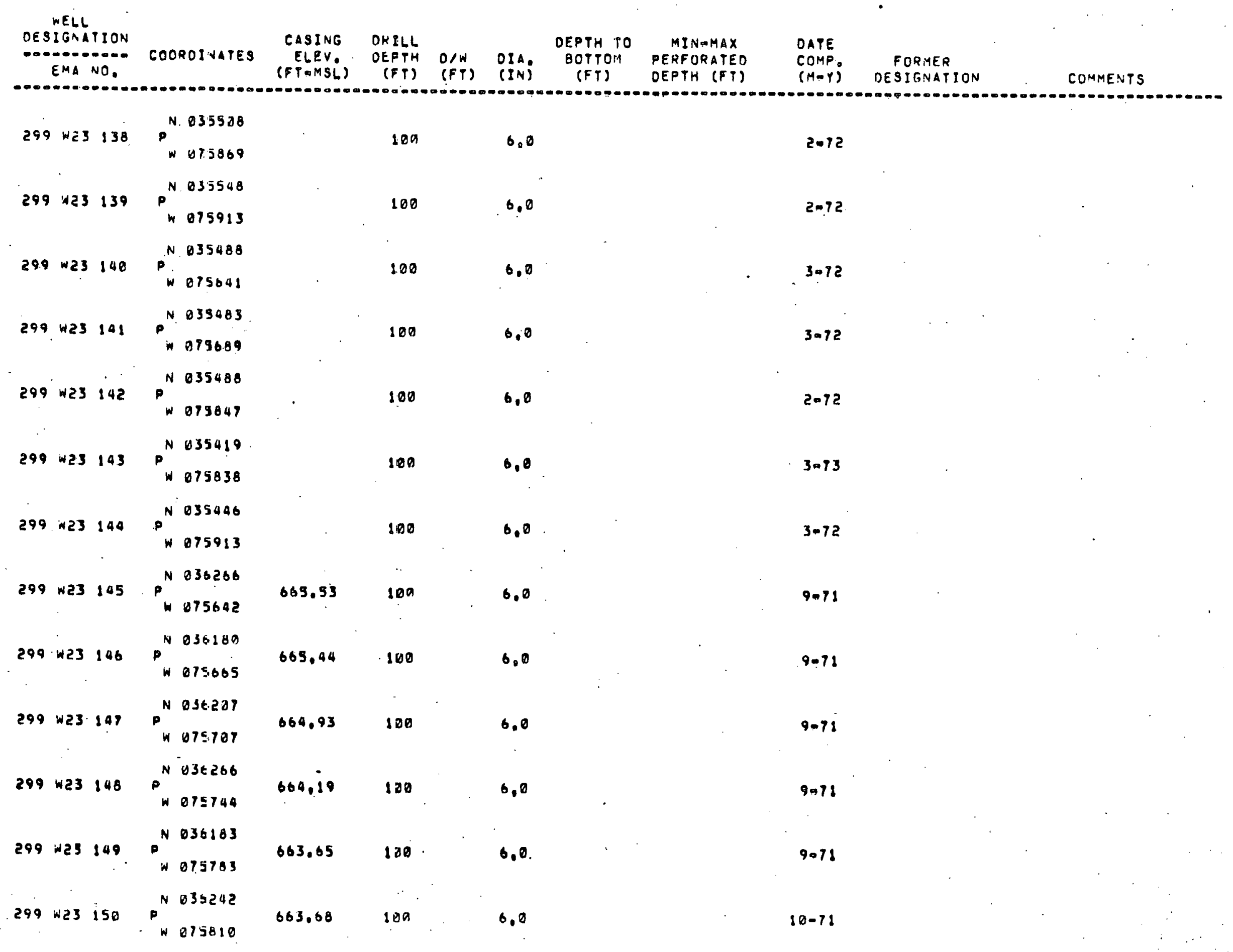




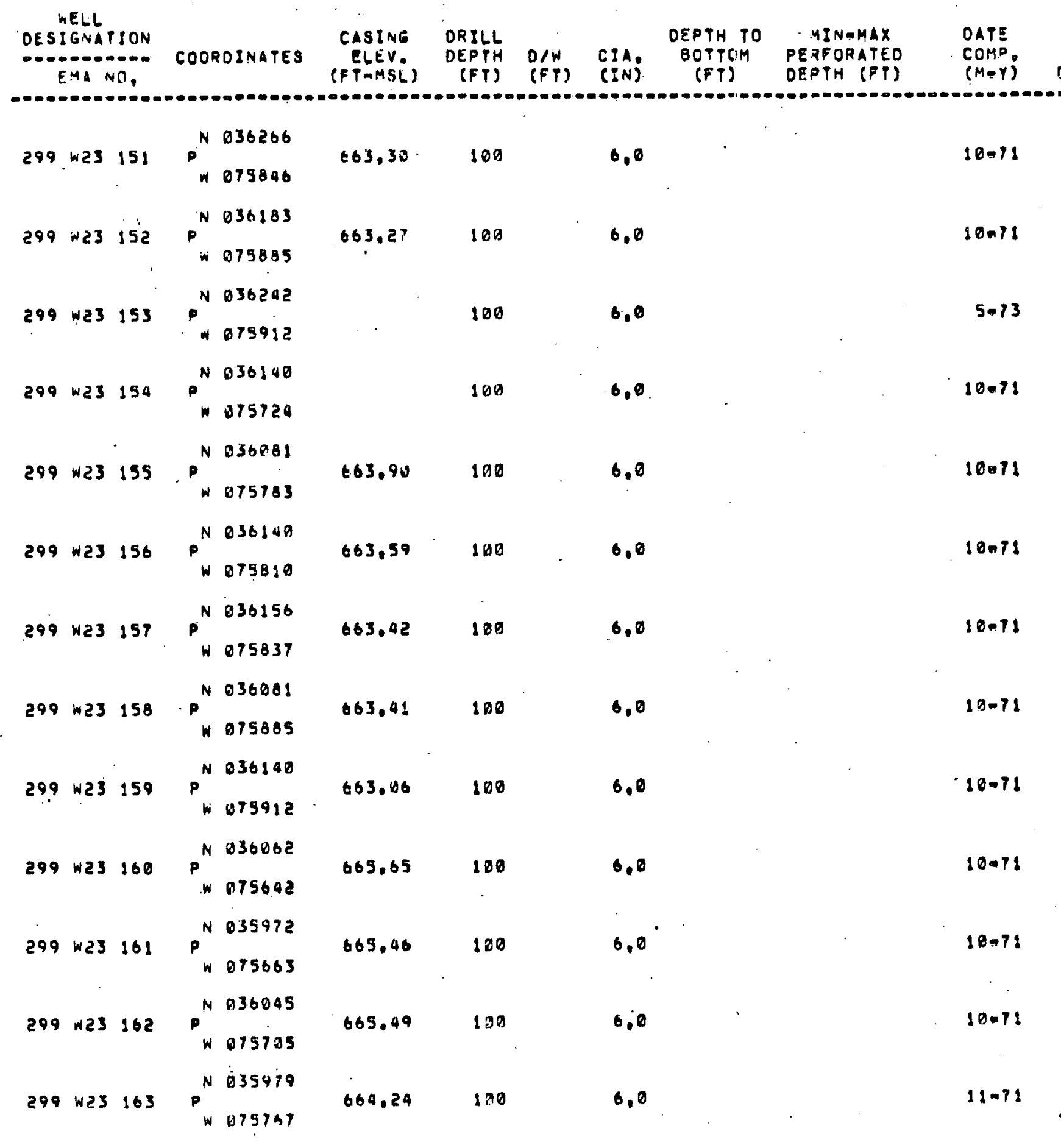




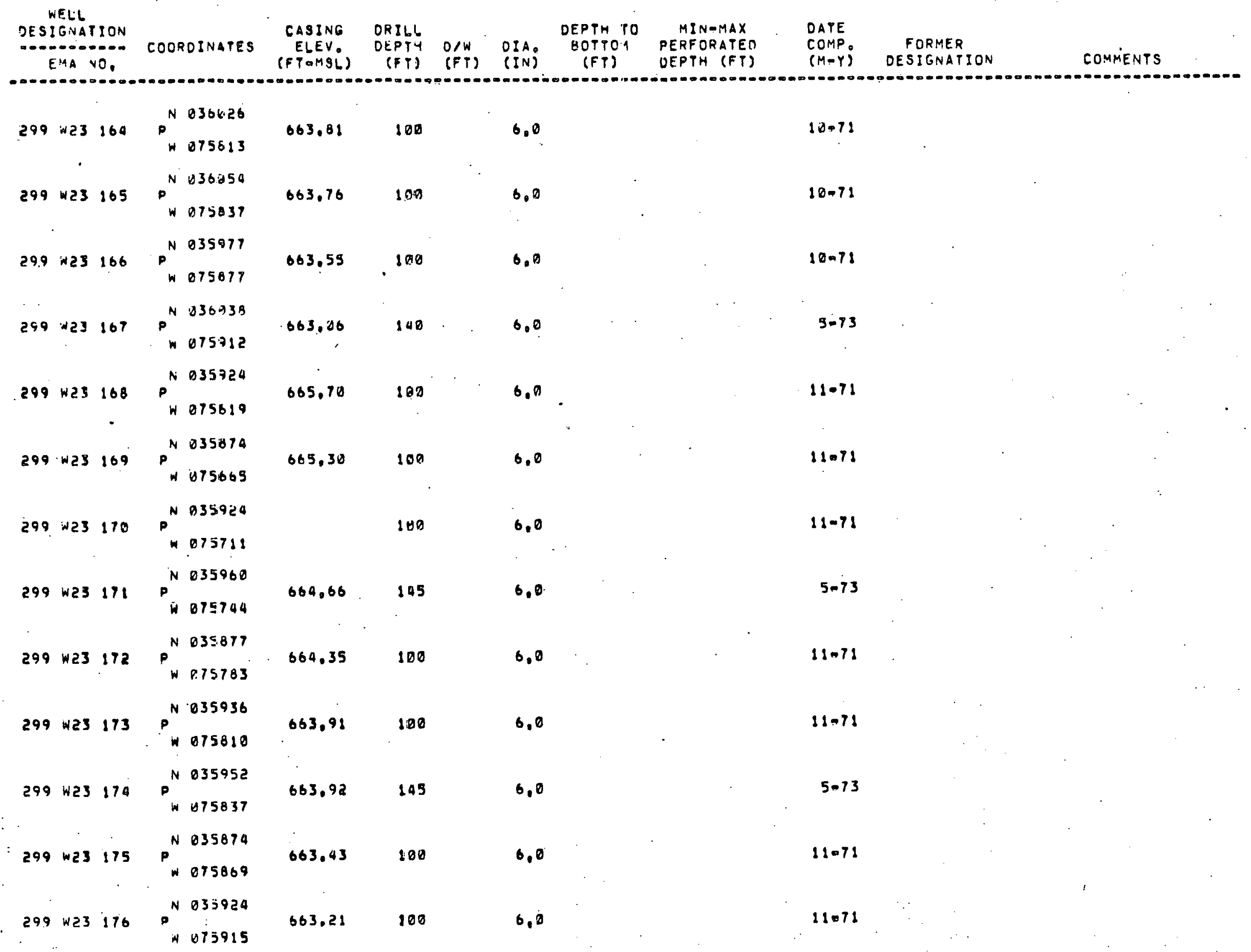


WELL

OESIGNATION

CASING DRILL

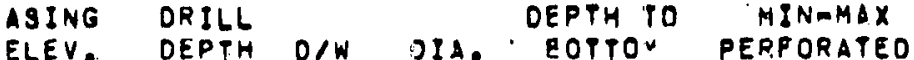

DATE

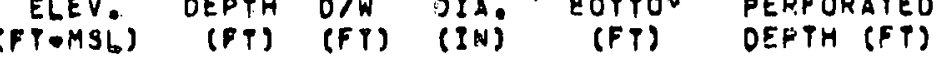

COMP FORMER

(M-Y) DESIGNATION

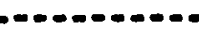

299 W23 179

665.48

On 0

299. $123 \quad 178$

665.92

On 0

$299+23119$

066.10

120

6.0

3074

299 W23 190

$665.07 \quad 109$

0,8

$3-79$

$299 \times 23 \quad 189$

664.10

190

6.9

$3-74$

299.พट) 182

665,24

100

6.0

$3 n 74$

299 W23 183

662.69

100

$6,8=$

3-79

299 W23 184

663.03

100

6,0

$3+74$

$299 W 23 \quad 185$

665,99

100

6.2

$3 \cdot 74$

299 N23 186

665.59

100

$B, 0$

$3=74$

299 W23 187

665.67

100

$b, 9$

$4 \cdot 74$

$299 \times 23 \quad 188$

100

$b, 0$

$2-74$

299 Wंड 189

h. 0

$2-74$ 
WELL

DESIGNATION

EMA NO.

COOROINATES

CASING

ORILL

DEPTH TO

MINAMAX
PERFORATED

DATE

ELEV OEPIH O/W OIA: BOTTOH PERFORATED

COMP. FORMER

$(M-Y)$ OESIGNATION

COMMENTS

$299 \times 23 \quad 190$

169

6.0

$11-74$

$299 \times 23191$

100

6.0

11074

$299 \times 23 \quad 192$

100

6,0

11.74

$299 \times 23 \quad 193$

100

0.0

10.74

$279 \times 23194$

100

6,0

10.74

$? 99 \times 23 \quad 95$

190

6,0

11074

299 W3 196

100

6.0

10.74

2द9 $123 \quad 199$

120

6.0

$11=74$

$290 \times 23198$

106

6.0

$18=74$

299 W2b
2915

650.00

87 DRY 8,0

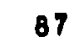

NONE

$6-54$

REDOX CRIB

NO.1 1

$299 \mathrm{H2B} 2$

650.00

100 DRY

8.8100

NONE

$6=54$

299 W2B $3 P^{N 033906}$

650.83

$190 \quad 168$

$8,0 . \quad 190$

NONE

6-54 REDUX CRIB

NO. 3

299 N2S

650,00

77

$6.0 \quad 77$

NONE

T-54 REOOX CRIO 


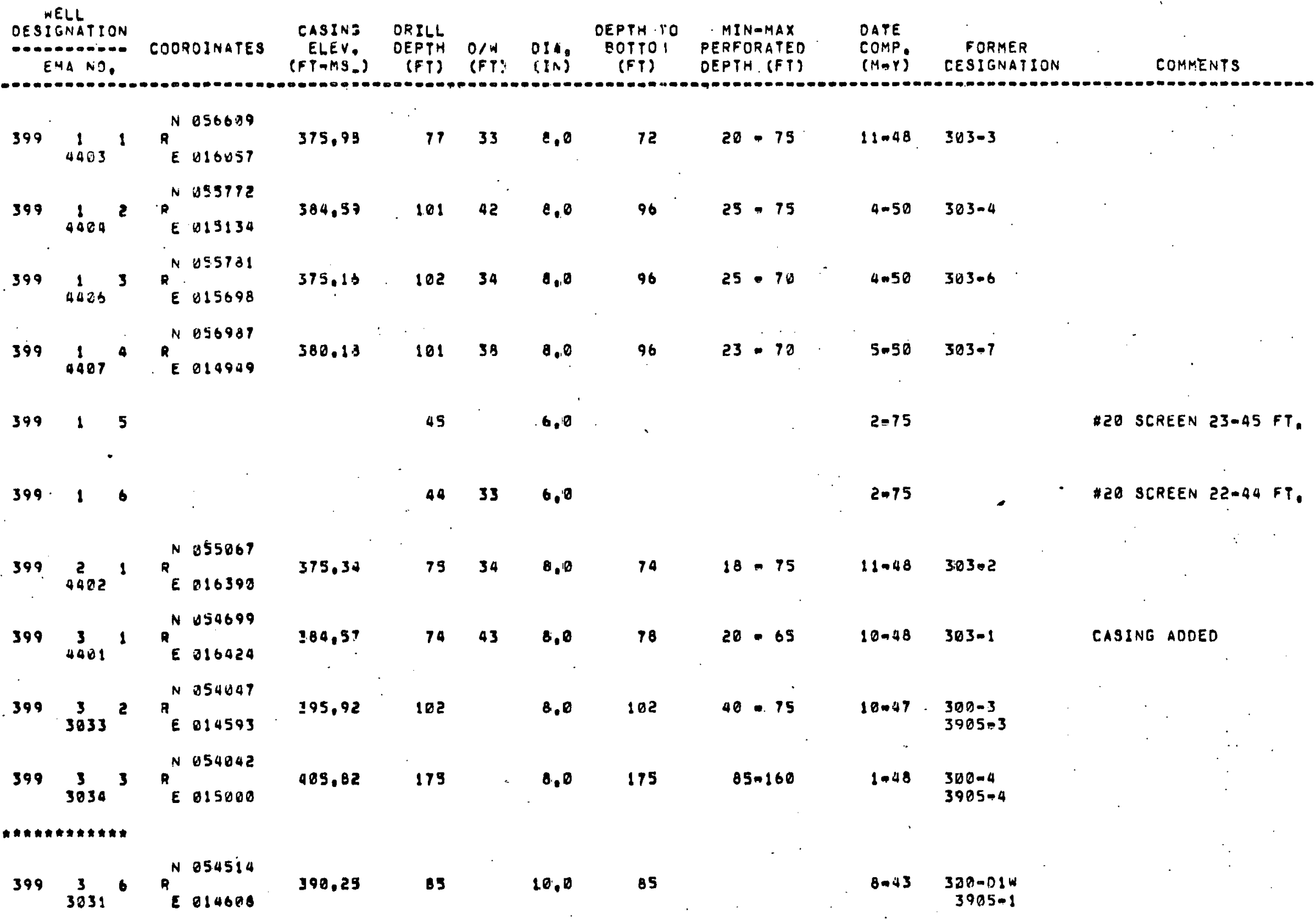




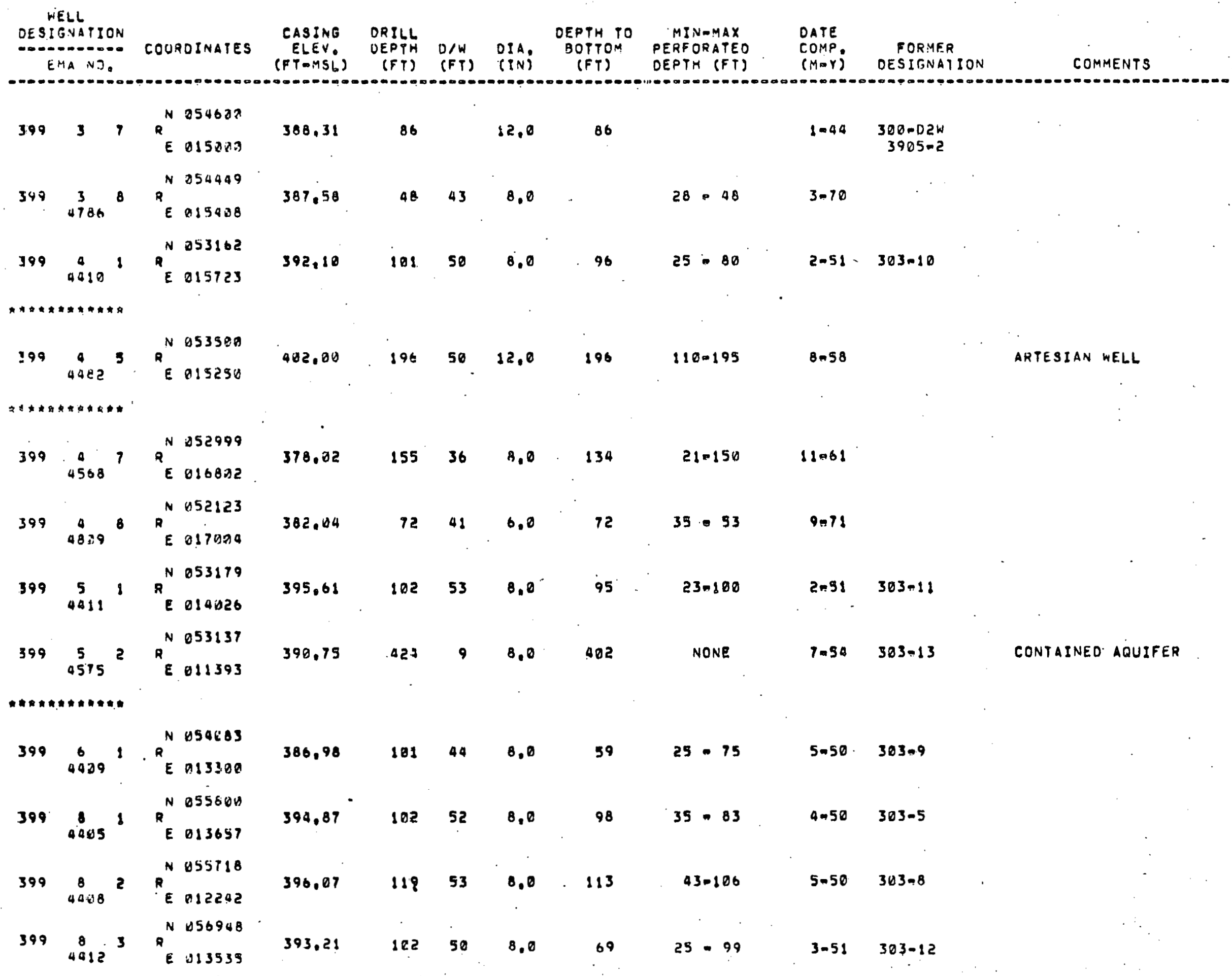




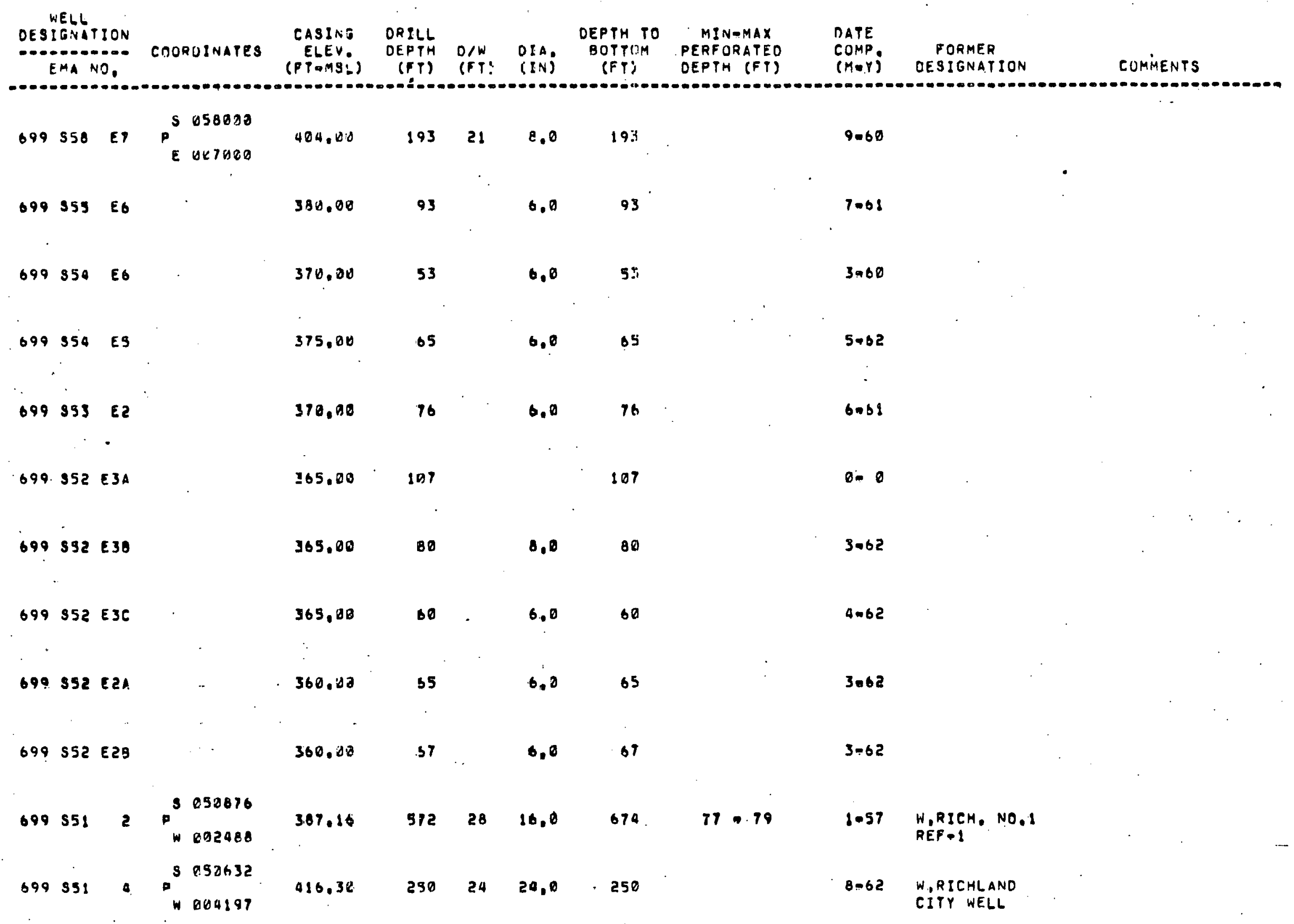




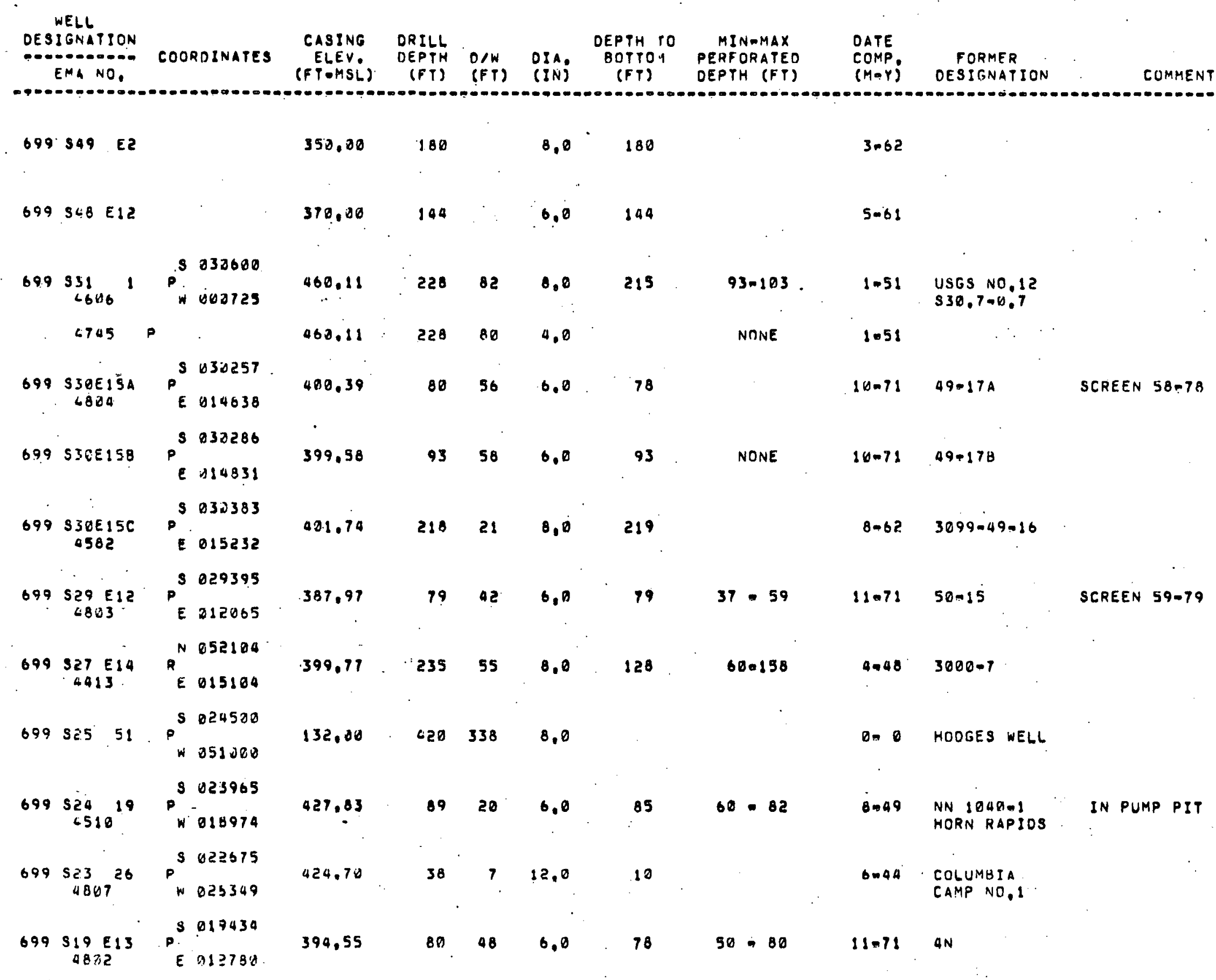




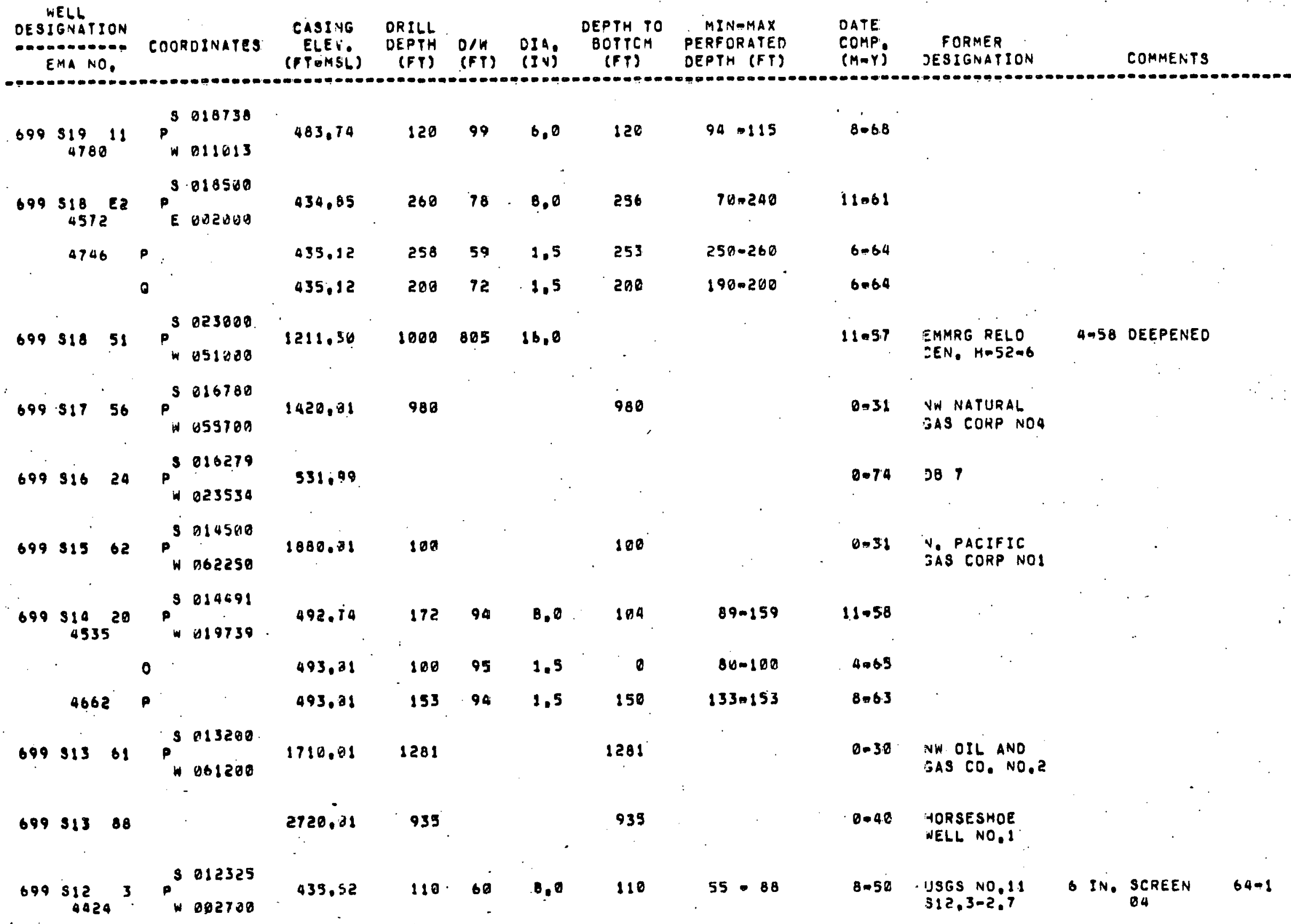




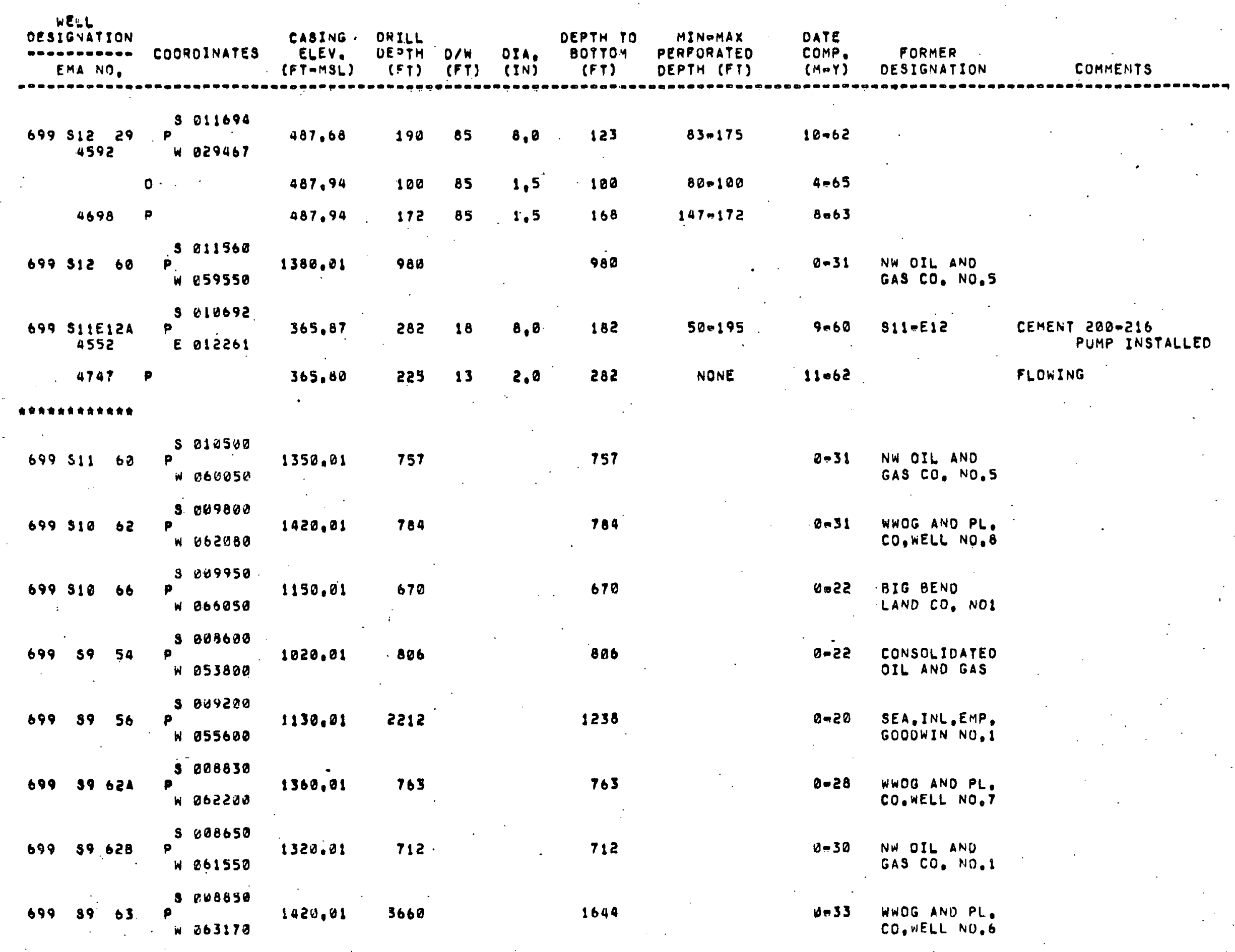




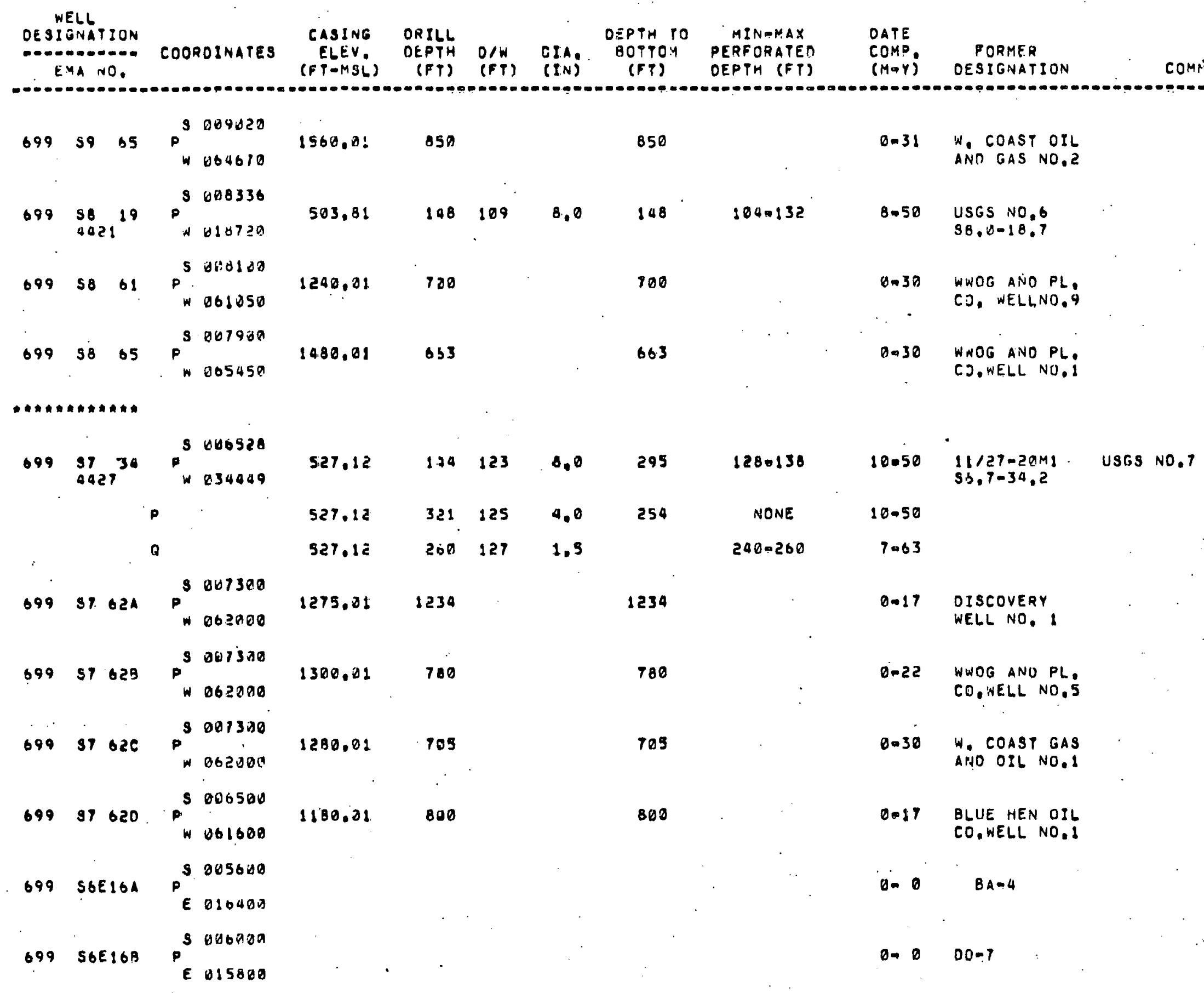




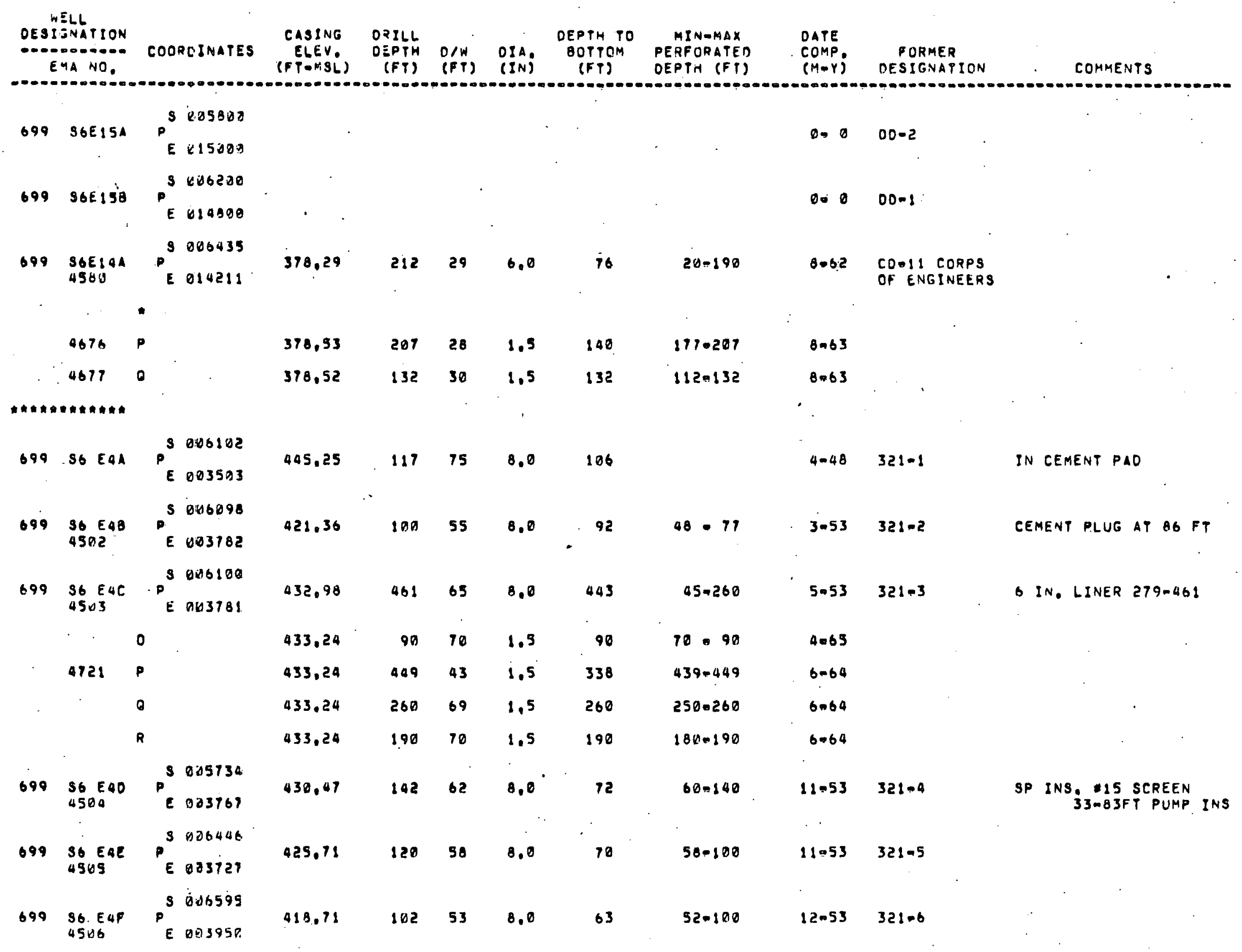




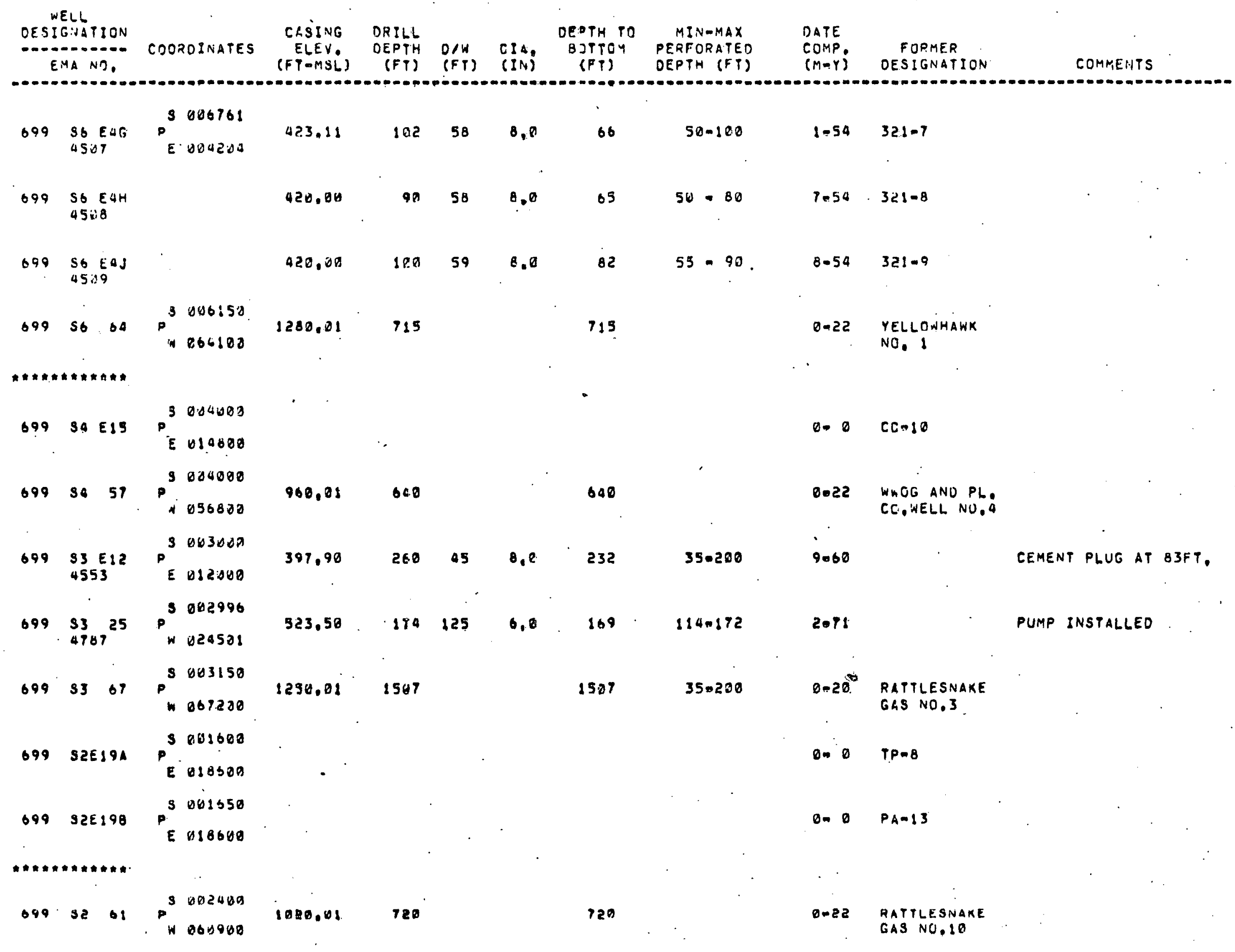


WELL

DESIONATION

COOROIVATES

CASING ORJ

DEPTH TO MINAMAX
ORJLL
OEPTH D/W DIA. BOTTEM PERFORATED DEPTH (FT)

DAPE

COMP. FORMER

EMa vo, (FTAML)

(FT) (FT) (IN) (FT)

(MOY)

FORMER
OESIGNATION

\begin{tabular}{|c|c|c|c|}
\hline 699 & SIEI9A & $P_{E}^{s}$ & $\begin{array}{l}001400 \\
219700\end{array}$ \\
\hline 699 & S1E19B & $P^{5}$ & $\begin{array}{l}03: 0110 \\
018070\end{array}$ \\
\hline 699 & SIEIQC & $P_{E}^{B}$ & $\begin{array}{l}001520 \\
0135100\end{array}$ \\
\hline
\end{tabular}

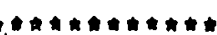

$6995170 \quad p^{8001008}$

$554.29 \quad 649 \quad 169^{\circ} \quad 0.0$

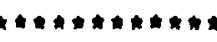

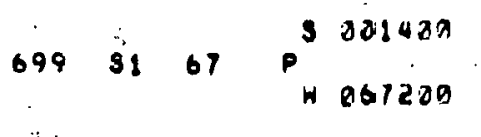

69930 ค P 500184

$1125.01 \quad 205$

$\begin{array}{llll}699 & 38,9000180 \\ 2817 & P^{5007479}\end{array}$

$699 \quad 0112$

$609110^{N 001300}$

$699 \quad 1100^{N^{-} 021420}$ 4513 W17593

$699210^{N \operatorname{\theta ic} 2459}$

W טuzads

$69923^{2} p^{N \operatorname{VR} 1900}$

548.68

10555

18.5

103. DRY
205

$230-380$

$12 m 69$

D. BA-5

Q-1 PA-14

D. D PA-15

RATTLESNAKE

3-72 FFTF WELL I WATER SUPPLY WELL

3.72 FFTF WELL 2 WATER SUPPLY WELL

A.58. RATTLESNAKE -22FT.018.5CASINGBE 3

Q: 0 IA-SPAI

$\begin{array}{llllll}537.68 & 350 & 142 & 0.0 & 160 & 109-265\end{array}$

D. $0 \quad 1 A-S P-2$

$477.14 \quad 147 \quad 90 \quad 8.0 \quad 119 \quad 85.123$
PUMP INSTALLED
8.50 USGS NO.B $1.9 \cdot 3.4$ 


\begin{tabular}{|c|c|c|c|c|c|c|c|c|c|c|c|}
\hline DES: & $\begin{array}{l}\text { ELL } \\
\text { GNATI } \\
\text { MANOE }\end{array}$ & & COJRUIVATES & $\begin{array}{c}\text { CASING } \\
\text { ELEV. } \\
\text { (FT-MSL) }\end{array}$ & $\begin{array}{l}\text { ORILL } \\
\text { OEPTH } \\
\text { (FT) }\end{array}$ & $\begin{array}{l}0 / w \\
(F+i)\end{array}$ & $\begin{array}{l}\text { OIA. } \\
\text { (IN) }\end{array}$ & $\begin{array}{l}\text { OEPTH TO } \\
\text { BOTTOMM } \\
\text { (FT) }\end{array}$ & $\begin{array}{l}\text { MINAMAX } \\
\text { PERFORATEO } \\
\text { DEPTH (FT) }\end{array}$ & $\begin{array}{l}\text { DATE } \\
\text { COMP, } \\
(M-Y)\end{array}$ & $\begin{array}{l}\text { FORMER } \\
\text { DESIGNAT }\end{array}$ \\
\hline 699 & $2 E$ & & $\begin{array}{l}P^{v 001633} \\
\equiv 013573\end{array}$ & 388.79 & & 2 & & & & 0.0 & \\
\hline 699 & $2 E$ & 19 & $\begin{array}{l}P^{Y 01962} \\
E 018926\end{array}$ & 384.24 & & & & & & 8.74 & $\mathrm{DE} \cdot 3$ \\
\hline 699 & $\begin{array}{c}2 \\
4526\end{array}$ & 33 & $\begin{array}{l}v 021813 \\
+032823\end{array}$ & 536.37 & 466 & 136 & 8.0 & 200 & 1360405 & $19-58$ & \\
\hline & & & 0 & 536.64 & 169 & 136 & 1.5 & 161 & 1480169 & $9=0$ & \\
\hline & 4654 & & $\mathbf{p}$ & 536.65 & 370 & 134 & 1,5 & 223 & $350-370$ & 8.63 & \\
\hline & 4659 & & 0 & 535.63 & 320 & & 1.5 & 319 & $300-328$ & 8.63 & \\
\hline & 4.656 & & $R$ & 536.65 & 263 & & 1.5 & 268 & $243-263$ & 8.63 & \\
\hline 699 & ${ }_{4593}^{3}$ & 45 & $\begin{array}{l}\text { v033049 } \\
+045099\end{array}$ & 504.54 & 175 & 98 & 8,8 & 178 & 90.100 & $10-58$ & \\
\hline 699 & 4 & 1 & $\begin{array}{l}P^{N} 003600 \\
N 001400\end{array}$ & & 107 & ORY & & & & $\theta=0$ & $1 A-3 P-3$ \\
\hline 699 & 4 & 5 & $\begin{array}{l}P^{N} 004180 \\
N 004908\end{array}$ & r & 106 & ORY & & & & 8. 0 & $1 Q-S P-2$ \\
\hline 699 & 4 & 6 & $\begin{array}{l}\therefore 003500 \\
=025600\end{array}$ & & 115 & ORY & & . & . & D. 0 & $18-5 P-1$ \\
\hline 699 & $\mathbf{9}$ & 2 & $\begin{array}{l}\text { v00475a } \\
+001800\end{array}$ & · & 207 & ORY & $\vdots$ & & . & 0.0 & $1 A-5 P-4$ \\
\hline 699 & $\mathbf{s}$ & 3 & $\begin{array}{l}\text { poas2an } \\
\text { N002100 }\end{array}$ & . & 106 & DRY & & & & Qn 0 & $18-59=4$ \\
\hline 699 & $\mathbf{3}$ & 4 & $\begin{array}{l}\text { p } \\
+0000000000\end{array}$ & 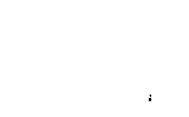 & 105. & DRY & - & & & $0-0$ & $18-5 P-3$ \\
\hline 699 & 6 & 8 & $\begin{array}{l}\text { v006300 } \\
{ }^{2} 0000000\end{array}$ & . & 3 & DRY & & & & $\theta-0$ & $18-5 P-6$ \\
\hline
\end{tabular}


WELL:

oESIGNATION

Designarion

EMA NO,

COOROINATES

CASING ORILL
ELEV. DEPYH TO DIH O/W OIA. BOTYOM PERFORATEO

(FT) (FT) (IN) (FT) DEPTH (FT)

DATE.

COMP FORMER

(M-Y) DESIGNATION

COMMENTS

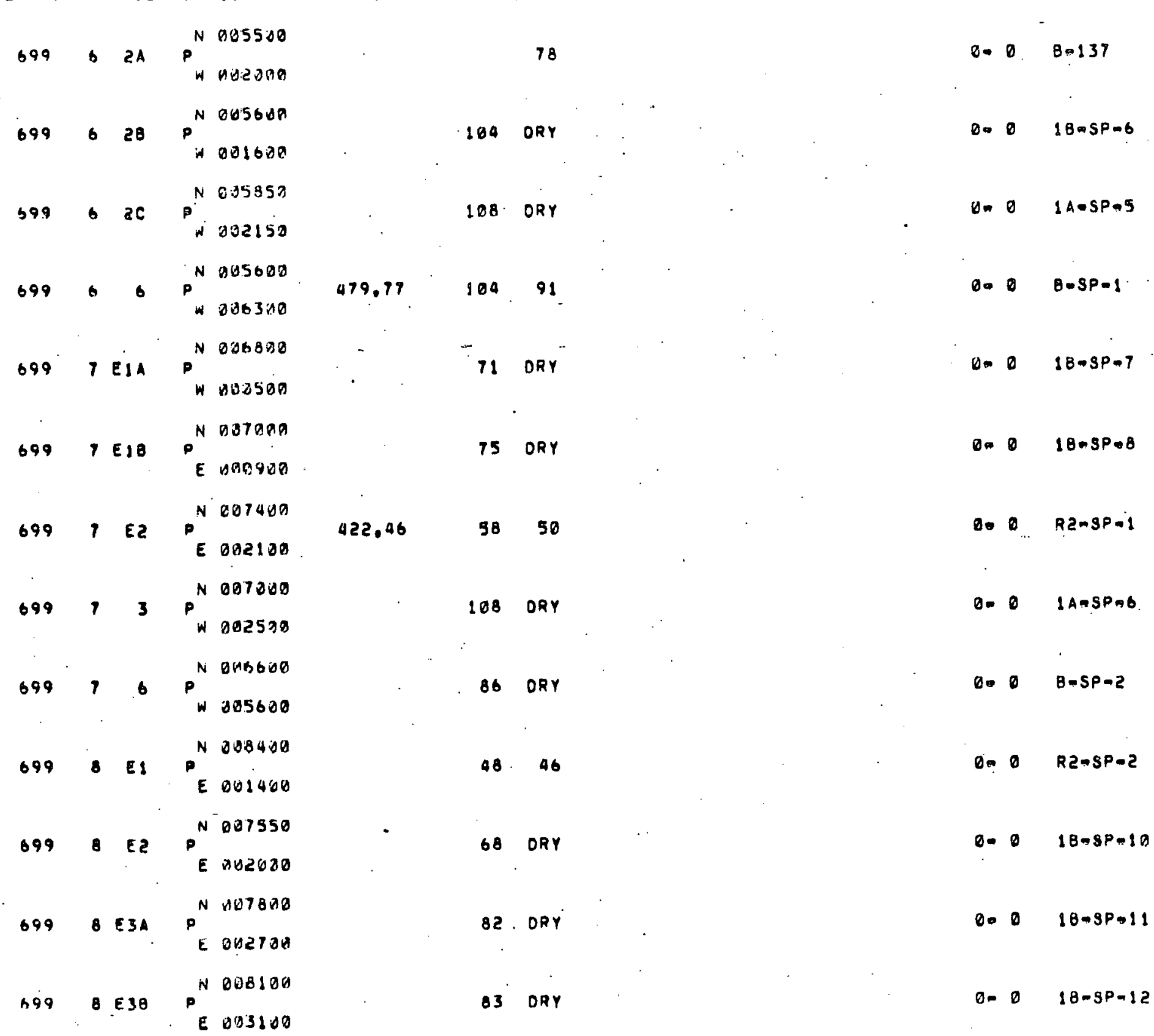




\begin{tabular}{|c|c|c|c|c|c|c|c|c|c|c|c|c|}
\hline OES & $\begin{array}{l}E L L \\
\text { GNATI } \\
\text { MA NO }\end{array}$ & & COUROINATES & $\begin{array}{l}\text { CASING } \\
\text { ELEV } \\
\text { (FTAMSL) }\end{array}$ & $\begin{array}{l}\text { DRILL } \\
\text { DEPYH } \\
\text { (FI) }\end{array}$ & $\begin{array}{ll}0 / 4 \\
(F+)\end{array}$ & DIA; & $\begin{array}{l}\text { DEPTH TO } \\
\text { BOTYD } 1 \\
\text { (FT) }\end{array}$ & $\begin{array}{l}\text { MINAMAX } \\
\text { PERFORATEO } \\
\text { OEPTH (FT) }\end{array}$ & $\begin{array}{l}\text { DATE } \\
\text { COMP, } \\
(M-Y)\end{array}$ & $\begin{array}{l}\text { FORMER } \\
\text { DESIGNATION }\end{array}$ & COMMENTS \\
\hline 699 & 8 & $\mathbf{s}$ & $\begin{array}{l}P^{N} 007700 \\
W 005000\end{array}$ & & 86 & DRY & & & & $\theta=0$ & $B-S P-3$ & \\
\hline$\cdots$ & antan & & & & & & & & & & & \\
\hline 699 & $\begin{array}{l}8 \\
442 \pi\end{array}$ & 17 & $\begin{array}{l}\text { Nogocida } \\
P^{W} 0.87125\end{array}$ & $5 \geq 2.44$ & 209 & 125 & 8,0 & 154 & $109-166$ & $5-50$ & $\begin{array}{l}\text { USGS NO,5 } \\
8,2-17,2\end{array}$ & $\begin{array}{r}\text { SCREEN 189-158FT P } \\
\text { LUG AT } 158 \text { FT. }\end{array}$ \\
\hline 699 & 4788 & $3^{25}$ & $\begin{array}{l}P^{N} 489995 \\
W 025073 .\end{array}$ & 589,30 & 170 & $\$ 10$ & 6.0 & 156 & 118.160 & 1.71 & & 6 IN. SCREEN 103-160 \\
\hline 699 & $\begin{array}{c}8 \\
4422\end{array}$ & $8^{32}$ & $\begin{array}{l}P^{N} \text { Me7875 } \\
\text { W } 031800\end{array}$ & 554.39 & 204 & 139 & 8.0 & 179 & $150-184$ & $9-50$ & $\begin{array}{l}\text { USGS NO.2 } \\
7.9-31.8\end{array}$ & 40 SCREEN $151-161$ \\
\hline 699 & 9 & E1 & $\begin{array}{l}P^{N} 009480 \\
3003680\end{array}$ & & 58 & a7 & 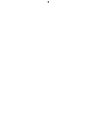 & & & $\theta-\theta$ & $R 2-S P=3$ & 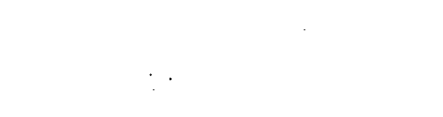 \\
\hline 699 & 9519 & $e^{E 2}$ & $\begin{array}{l}P^{N} 003579 \\
E 002324\end{array}$ & 418.89 & 454 & 49 & $b, 0$ & 60 & $15=255$ & 1.58 & $699-9 \cdot 5.4$ & $\begin{array}{l}\text { PUMP AT } 324 \text { FT, PLUE } \\
\text { AT GOFT, PUMP INS }\end{array}$ \\
\hline 699 & 9 & Es & $\begin{array}{r}P_{E}^{N} 009148 \\
E 05120\end{array}$ & & & 84 & & & & $\theta-\theta$ & & \\
\hline 699 & 9 & 3 & $P^{N}$ W003600 & & 116 & ORY & & & & $\theta \cdot 0$ & $1 A-S P=7$ & \\
\hline 699 & 9 & aA & $\begin{array}{l}P_{E}^{-N} 04.8700 \\
E 8150\end{array}$ & & 86 & DRY & & & & 0.0 & $18-5 P-13$ & \\
\hline 699 & 9 & 48 & $\begin{array}{l}P_{\text {W }}^{N} 04086000 \\
P^{2}\end{array}$ & - & 17 & 73 & & & & 0.0 & $B=3 P=4$ & . \\
\hline 699 & 10 & 0 & 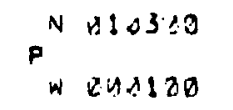 & & 50 & 46 & & & & $\theta .0$ & $R 2-S P-4$ & $\therefore$ \\
\hline 699 & 18 & 34 & $P_{N}^{N} 033466$ & & 573 & 57 & & & & $\theta=0$ & $8-36$ & \\
\hline
\end{tabular}


WELL

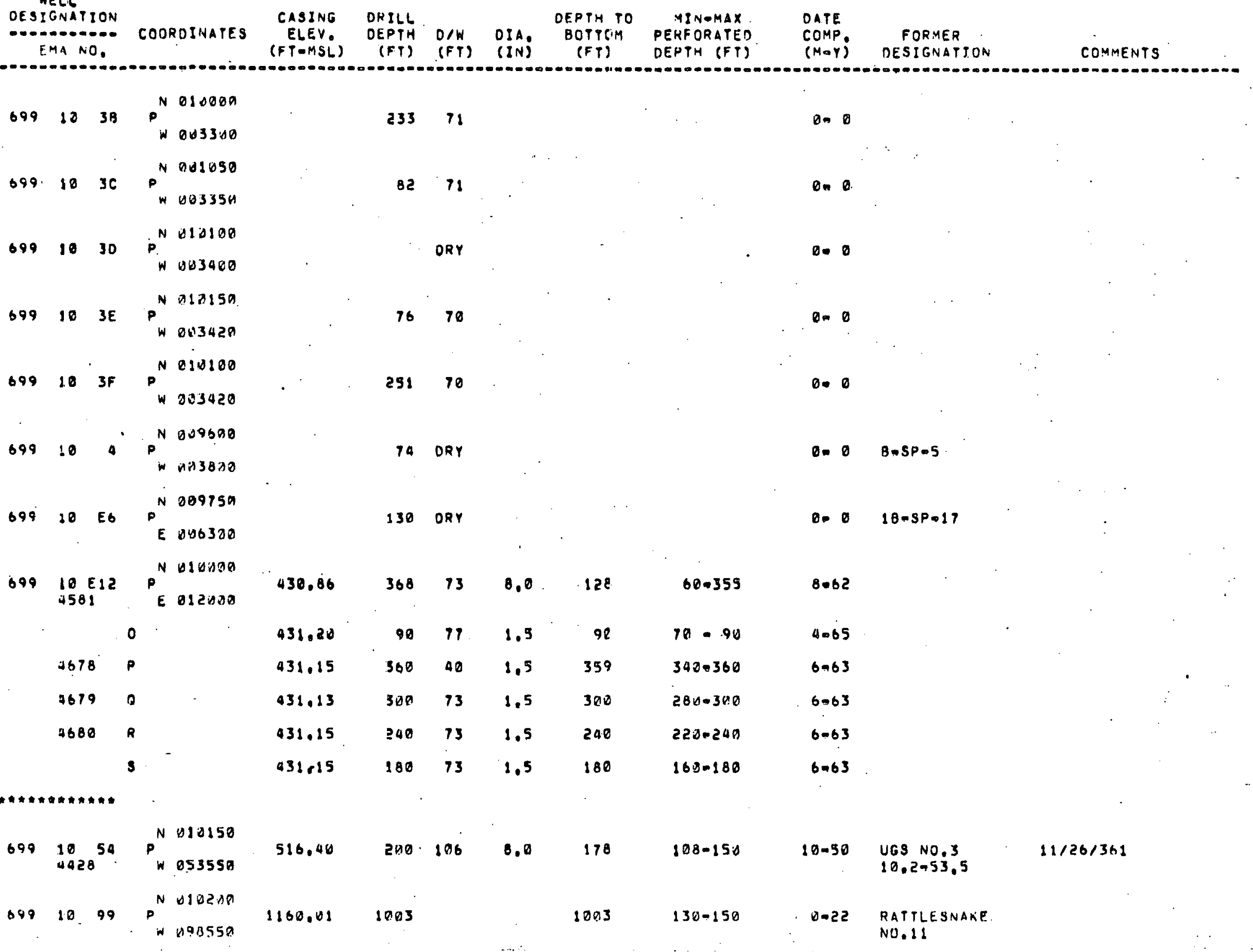




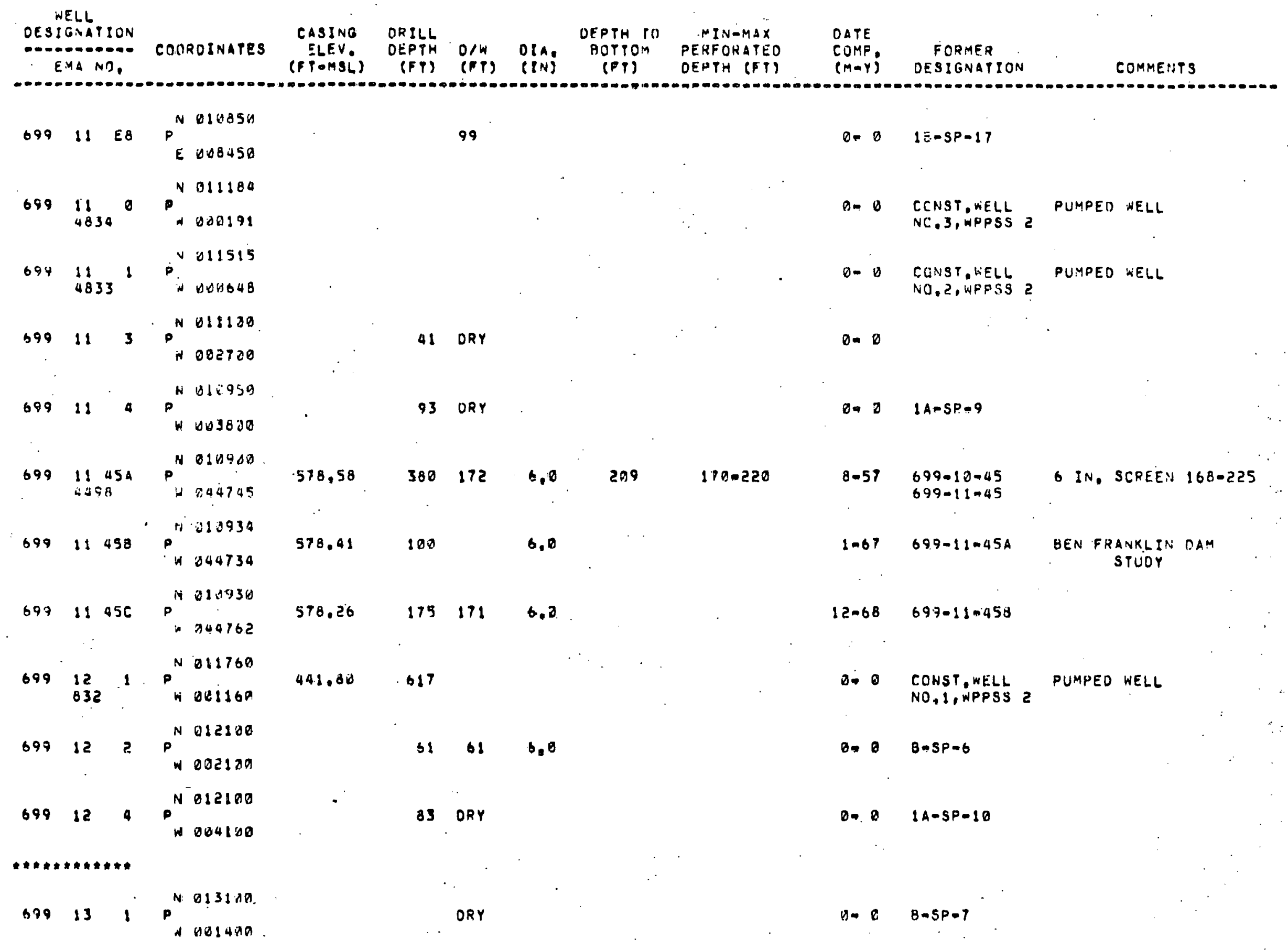




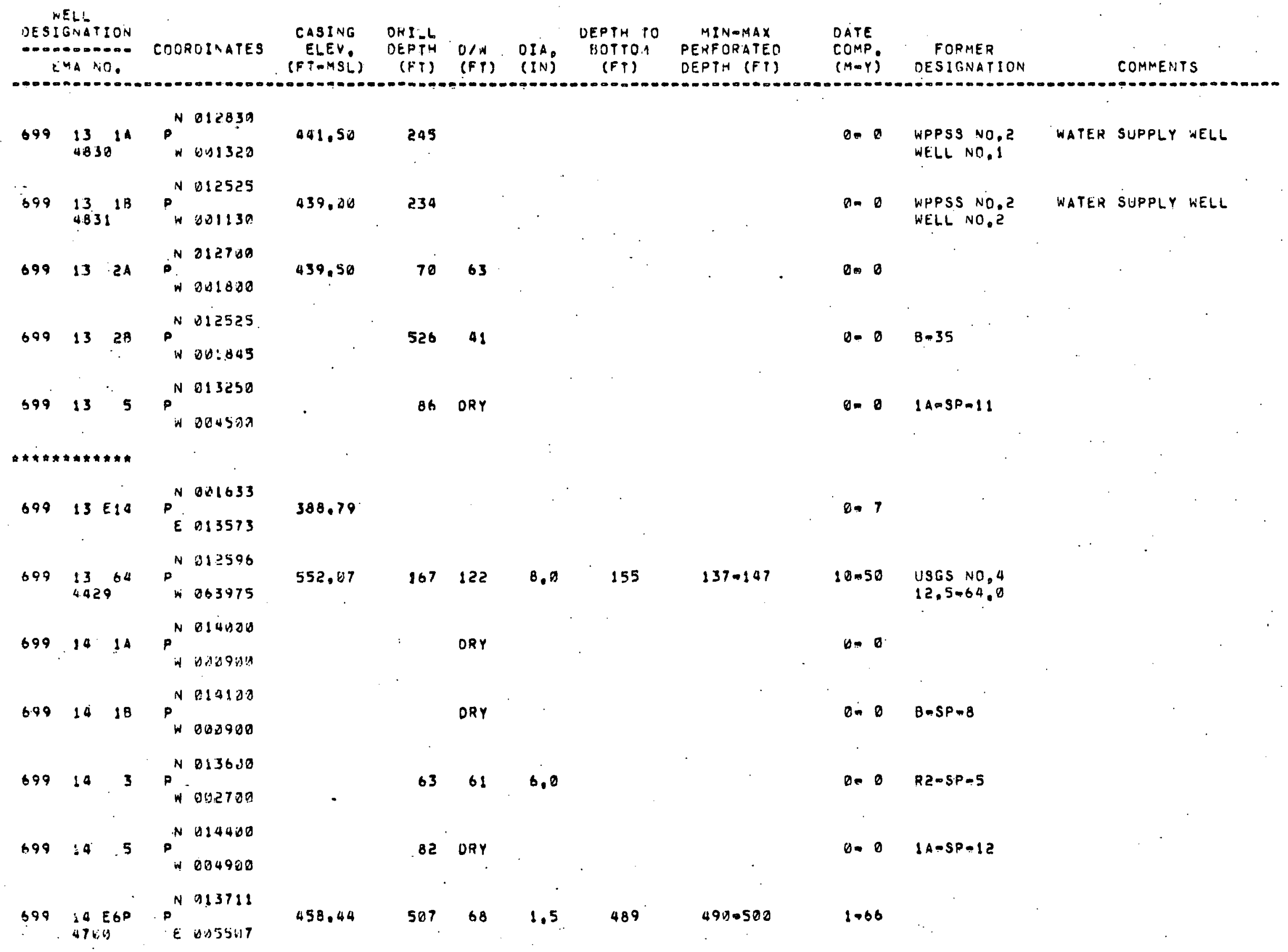


WELL

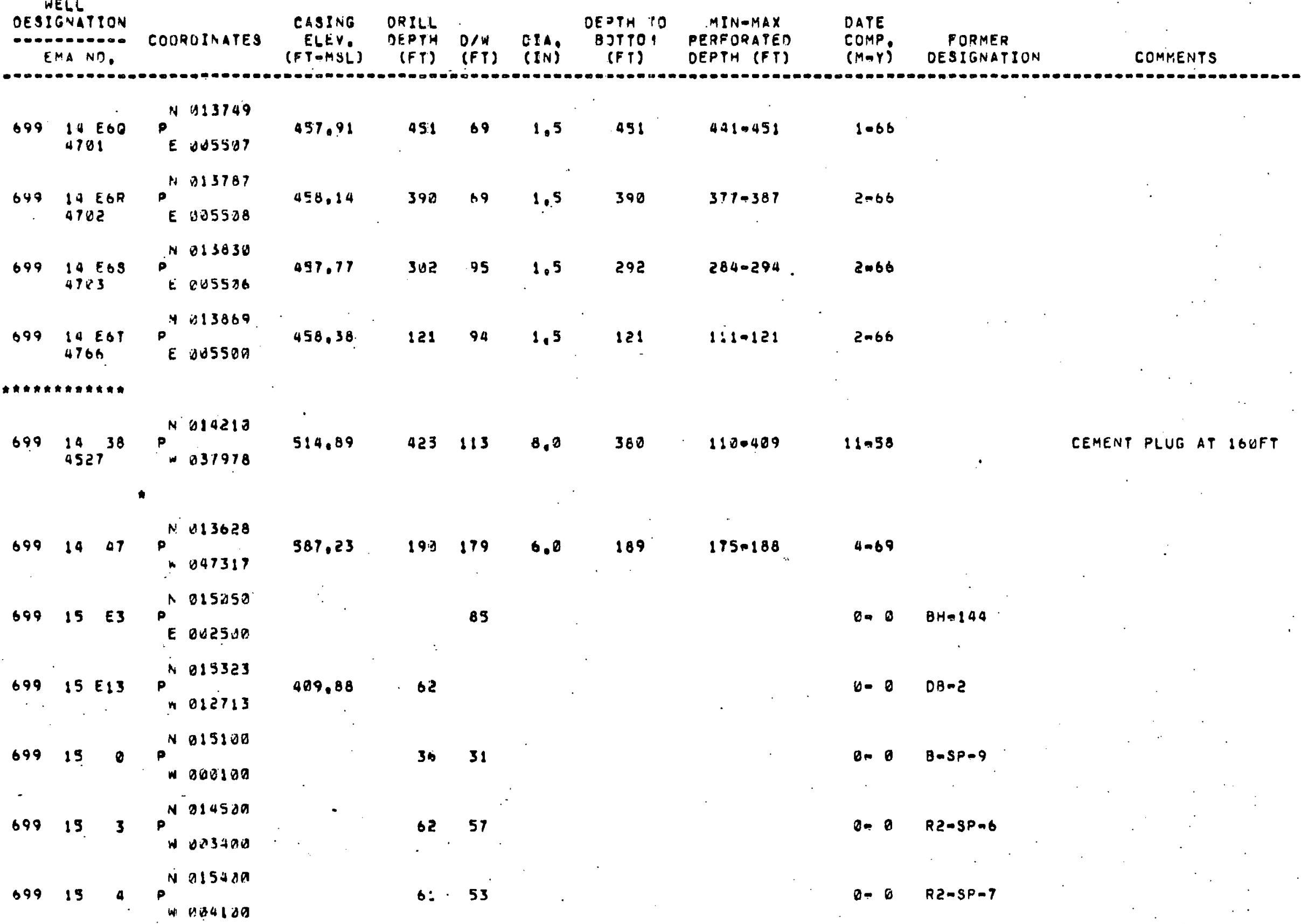


WELL

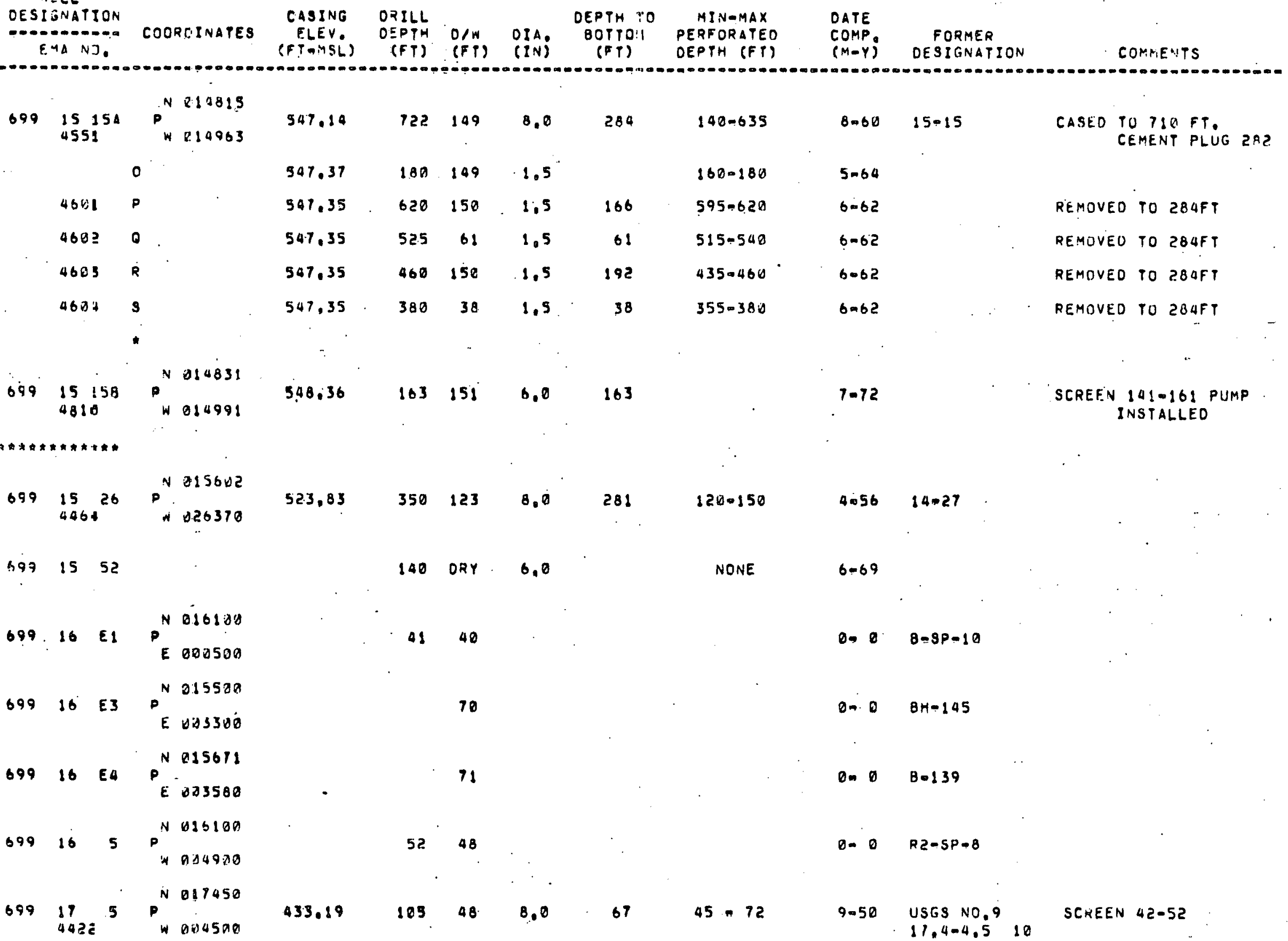




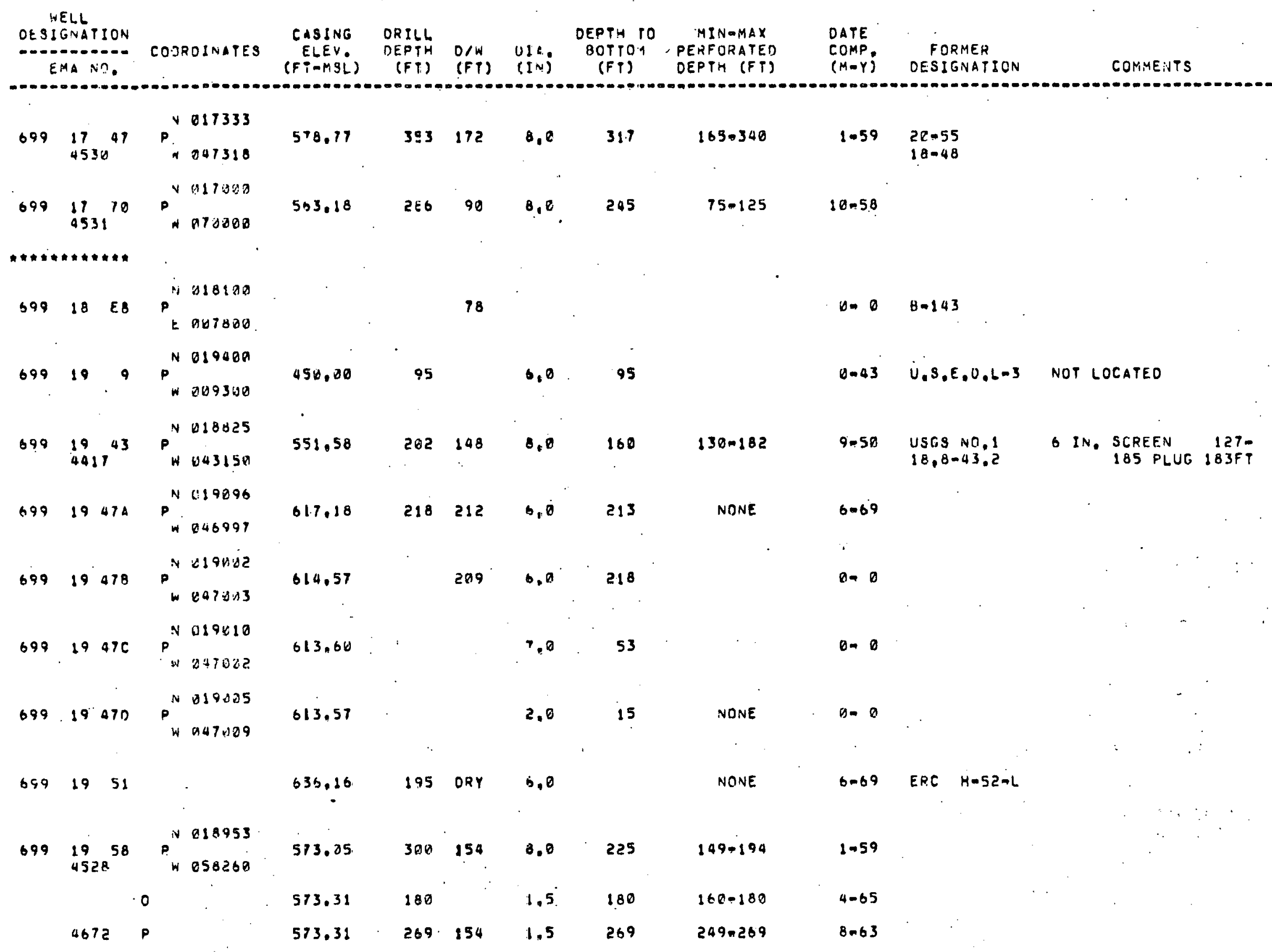




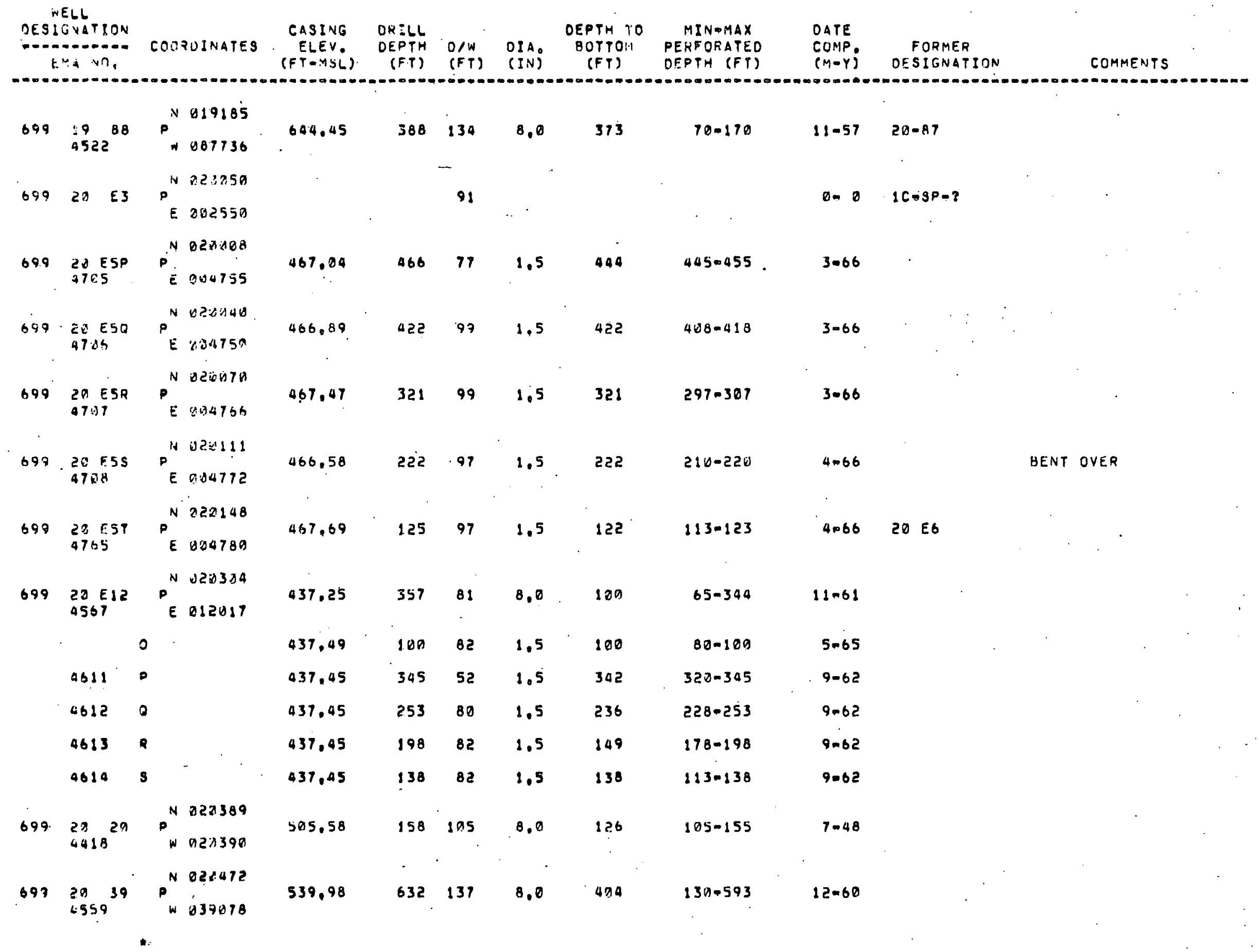




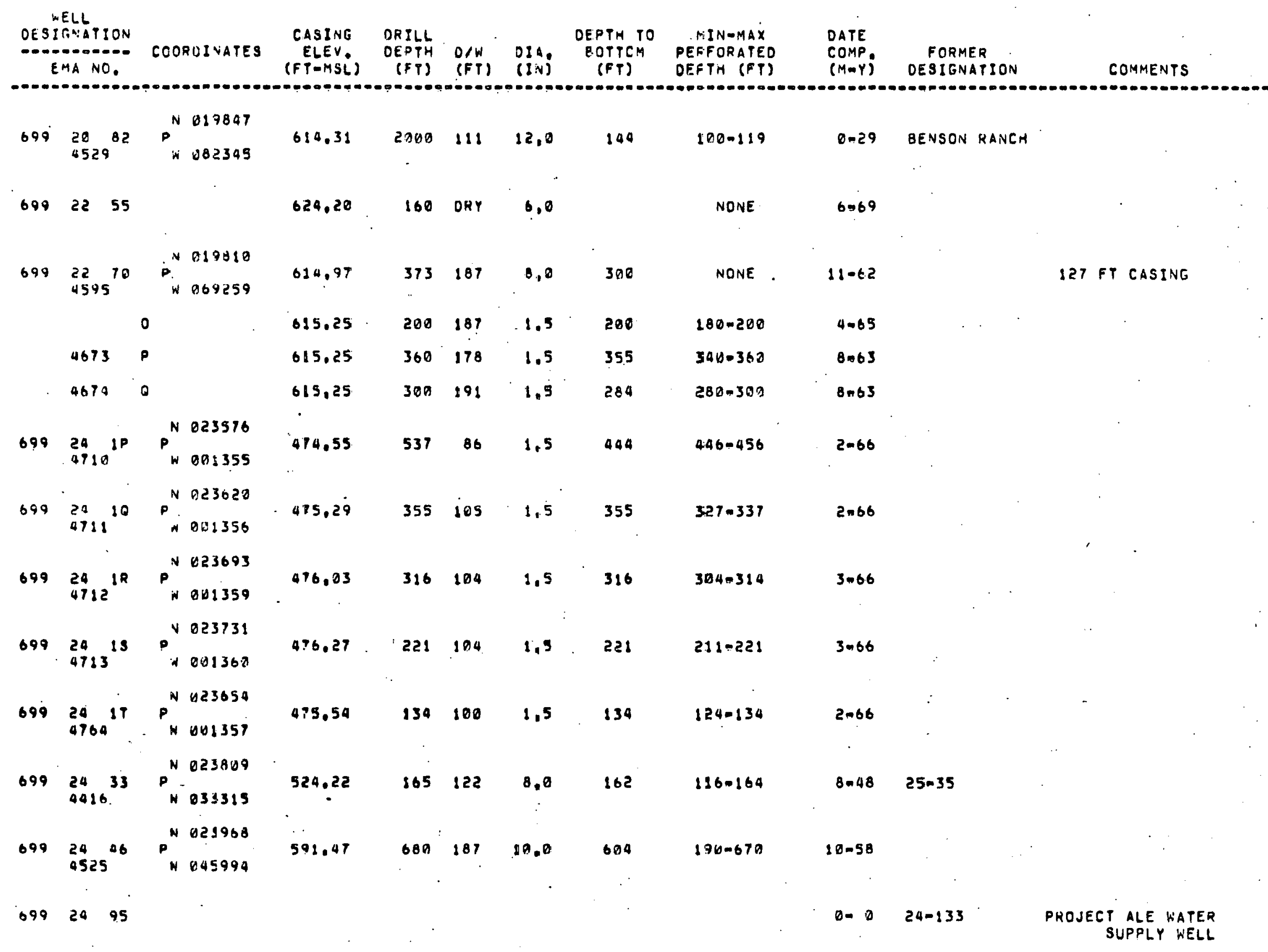


WELL

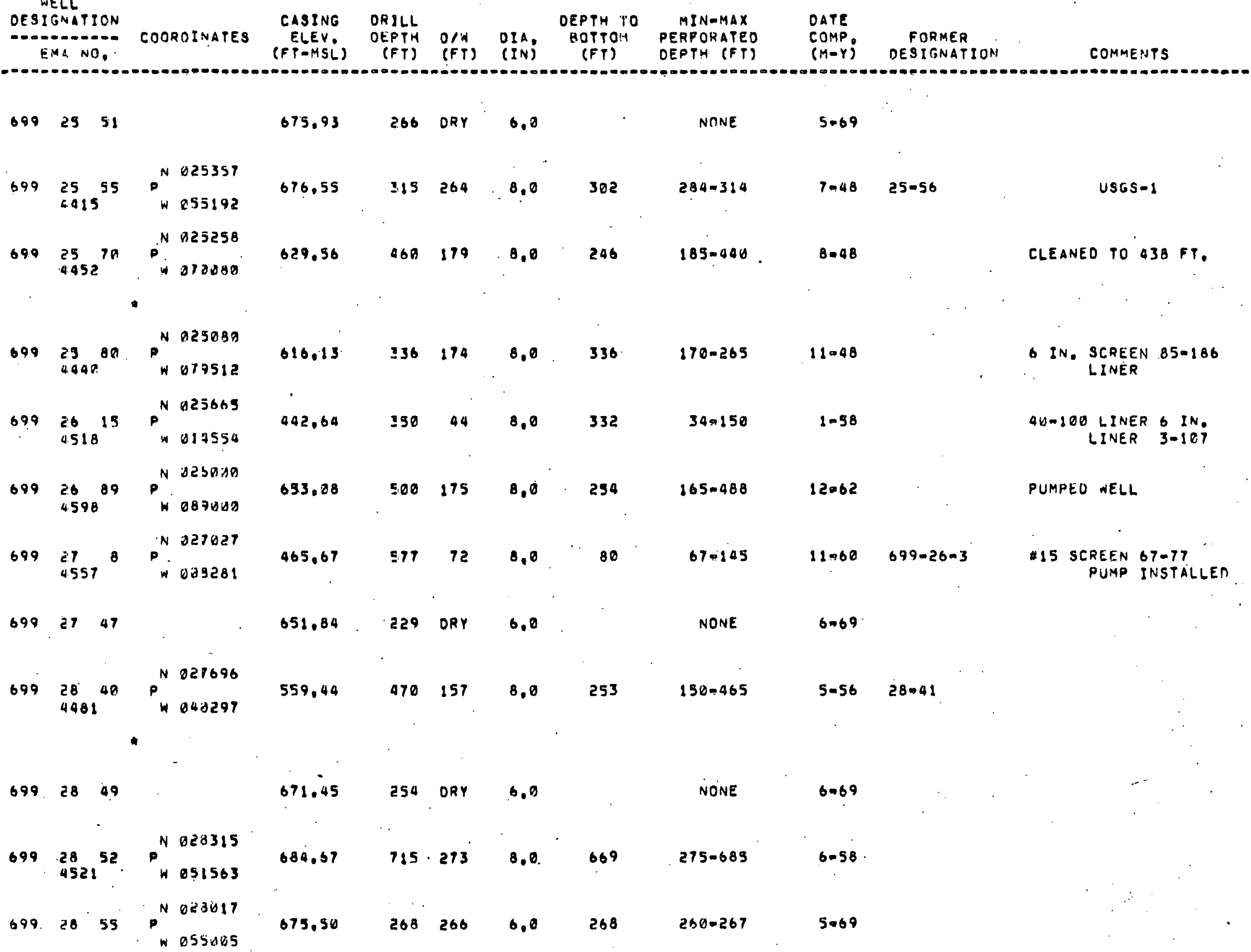




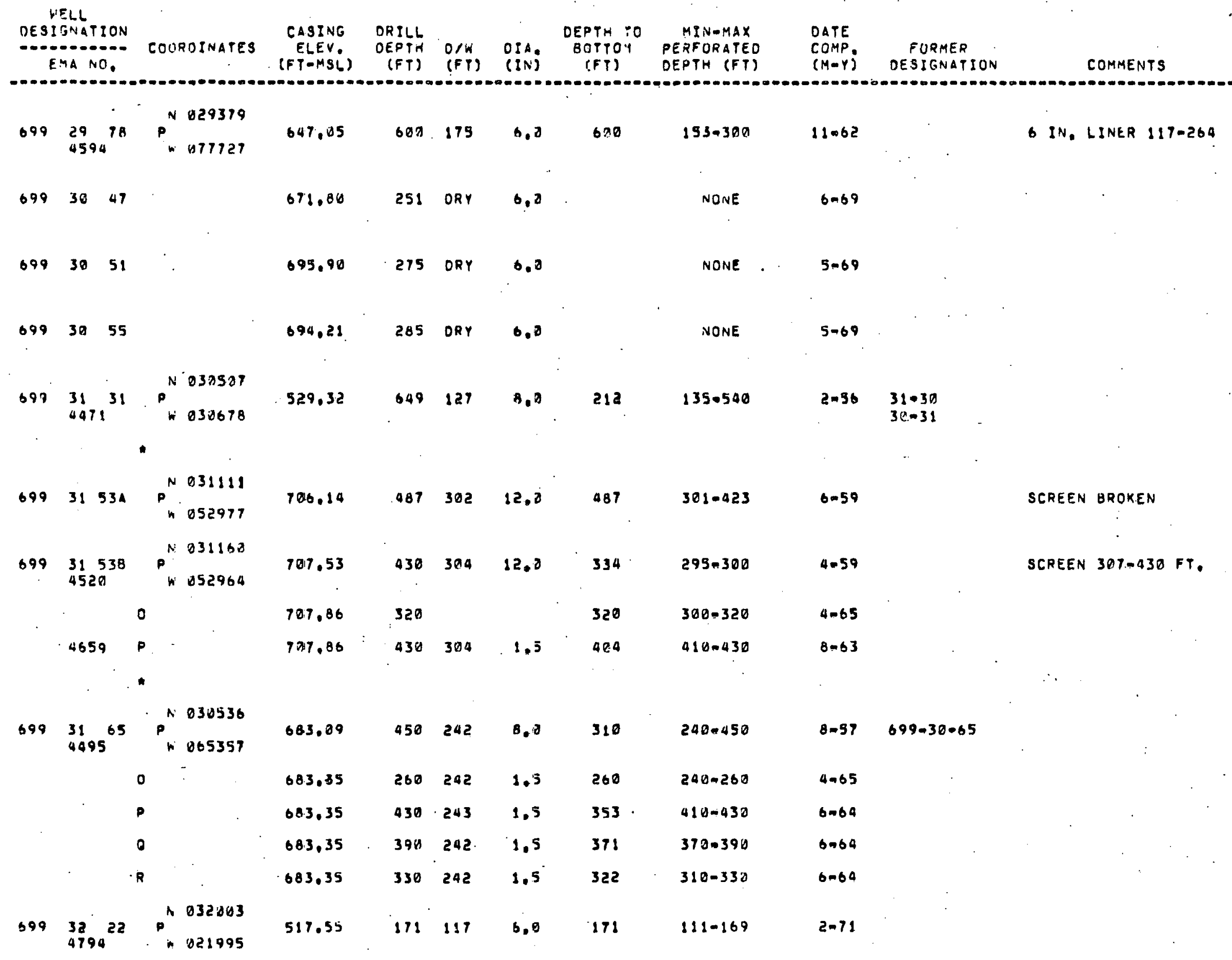


WELL

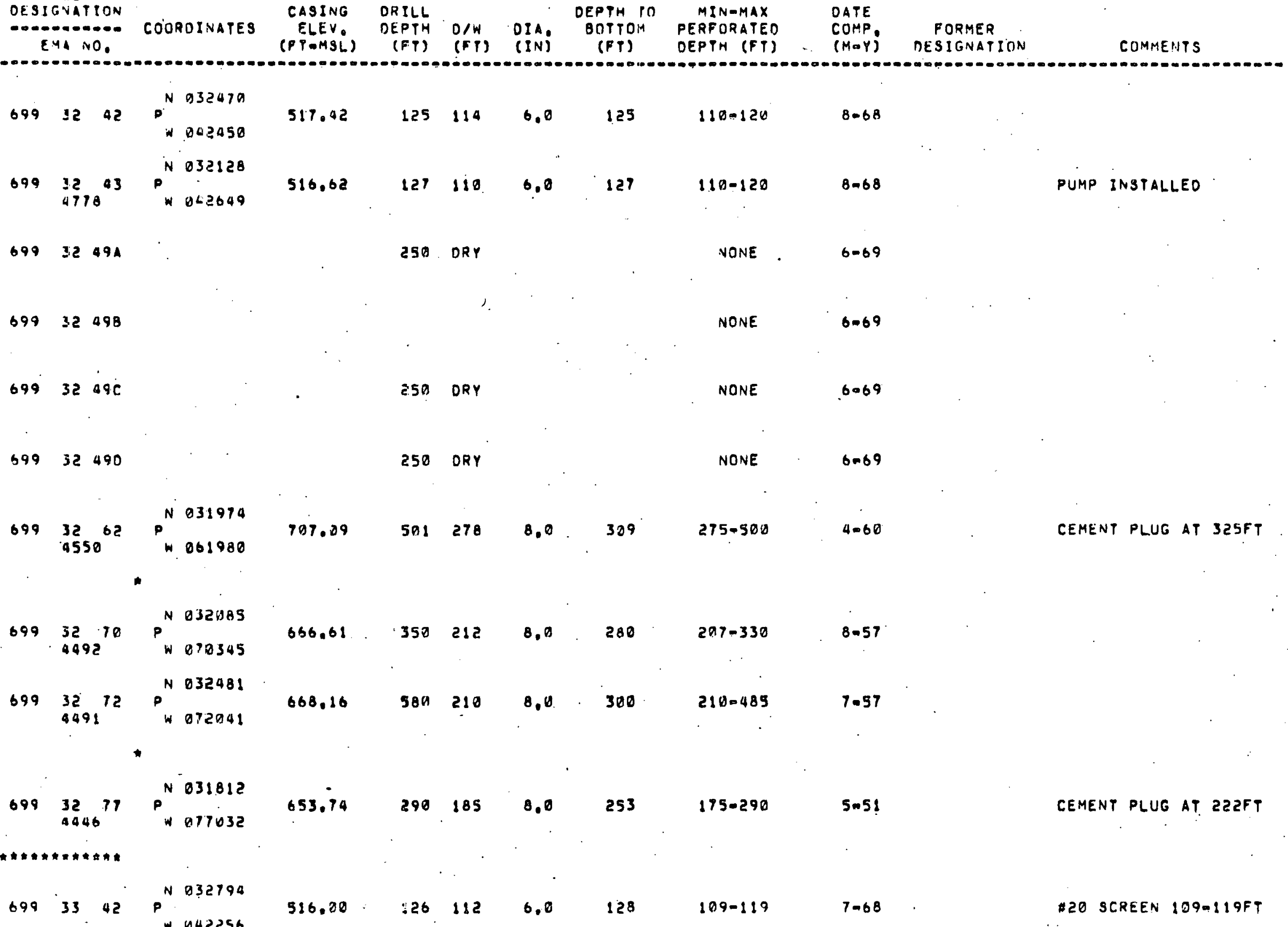




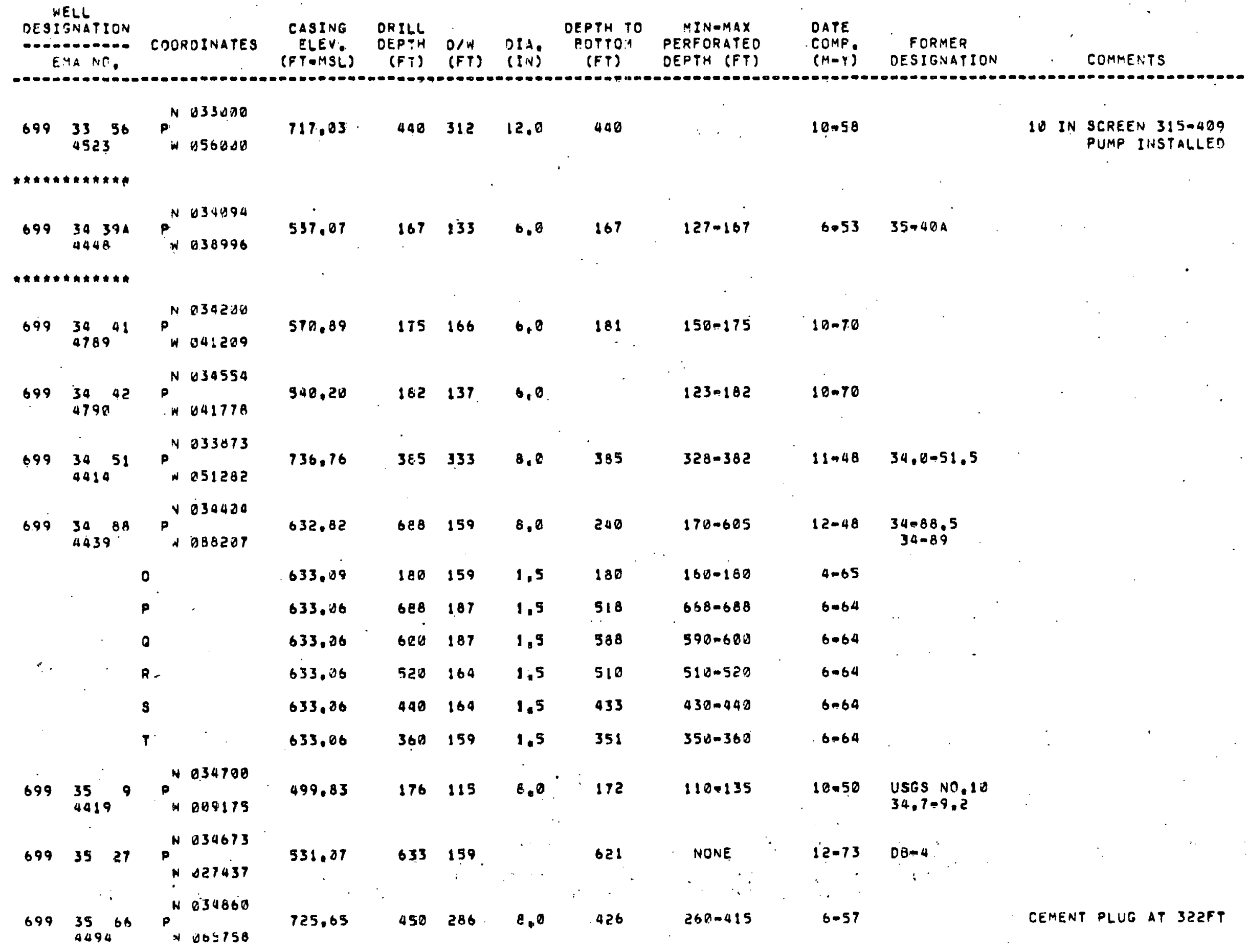


WELL

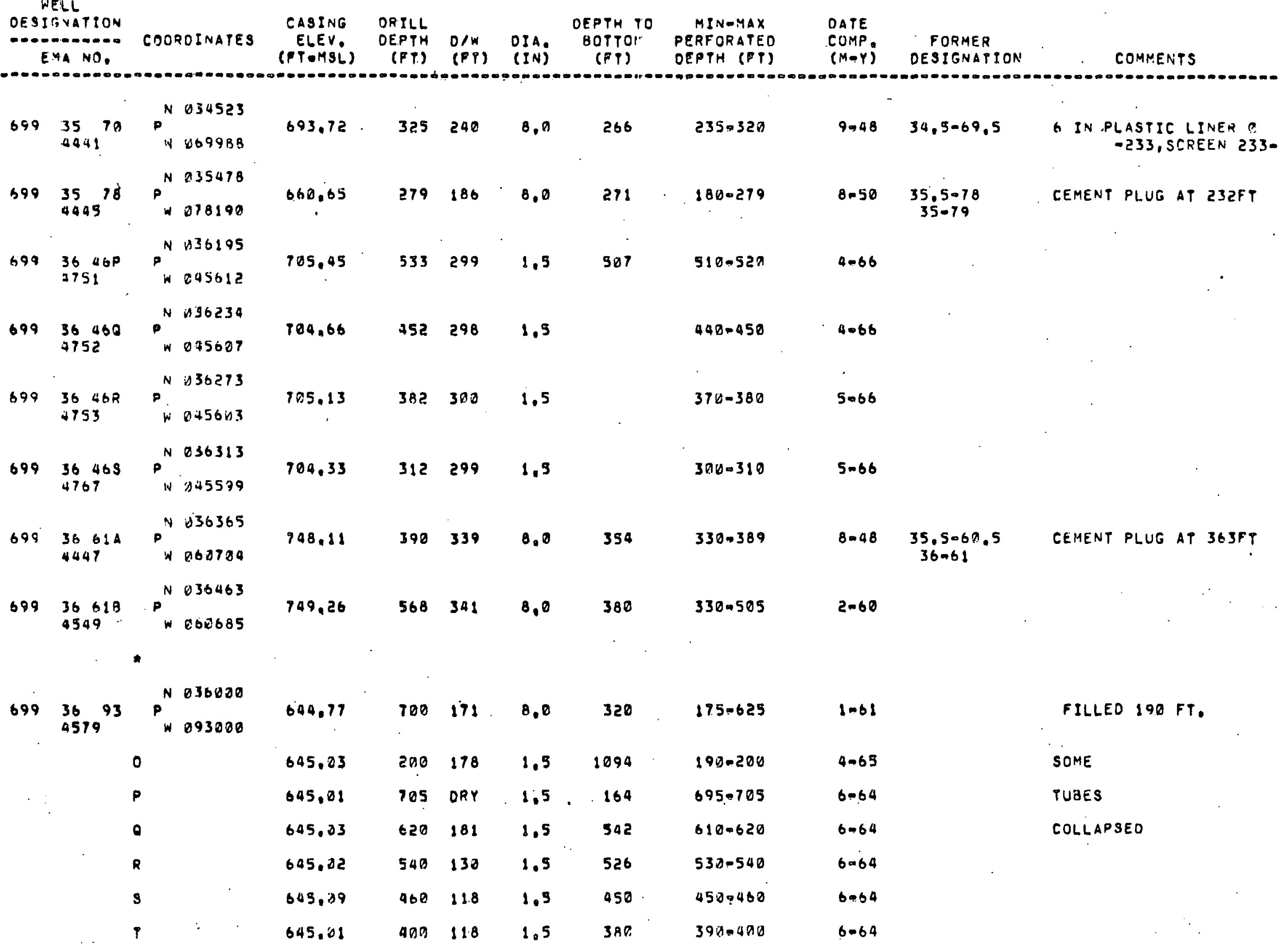




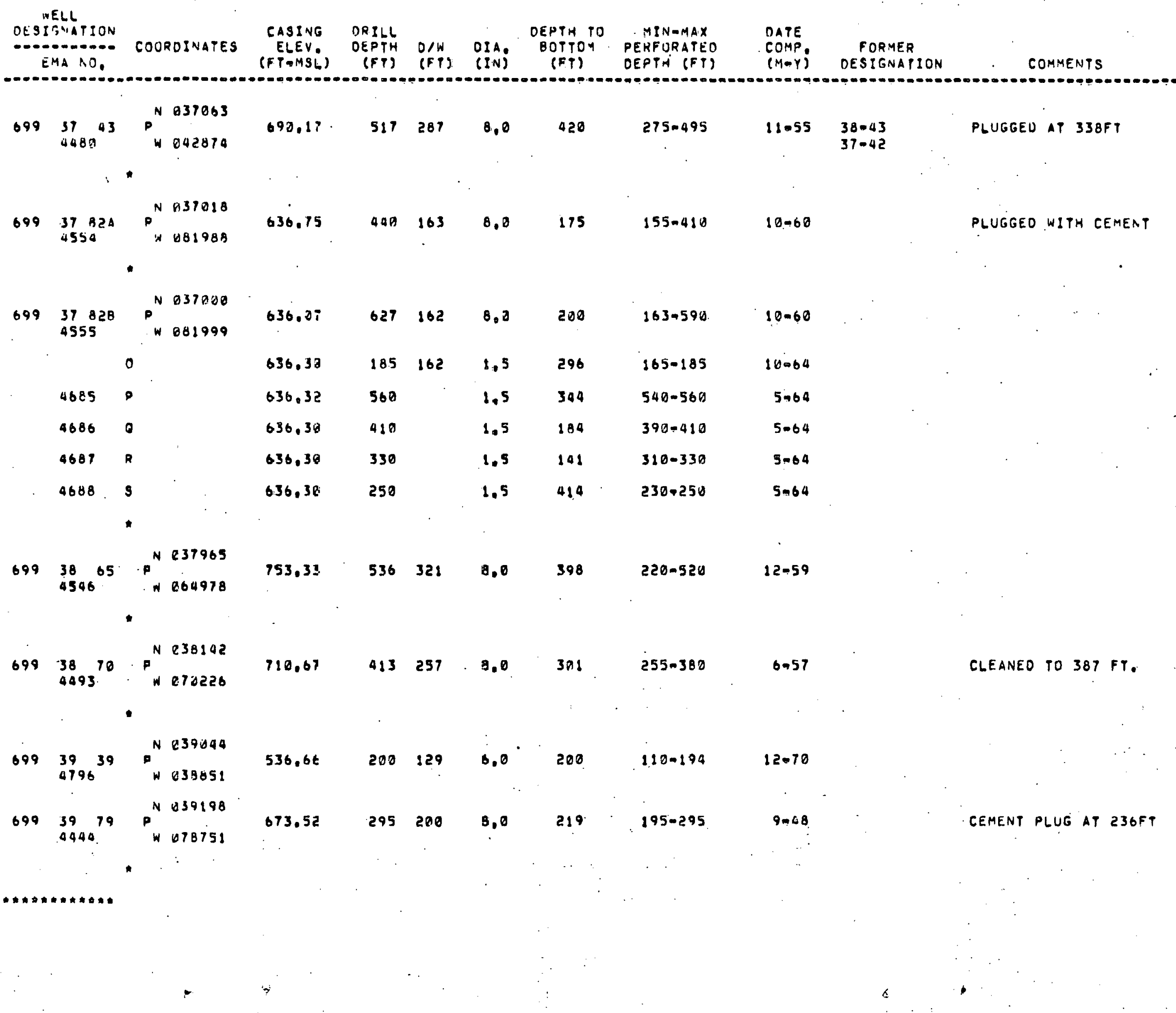




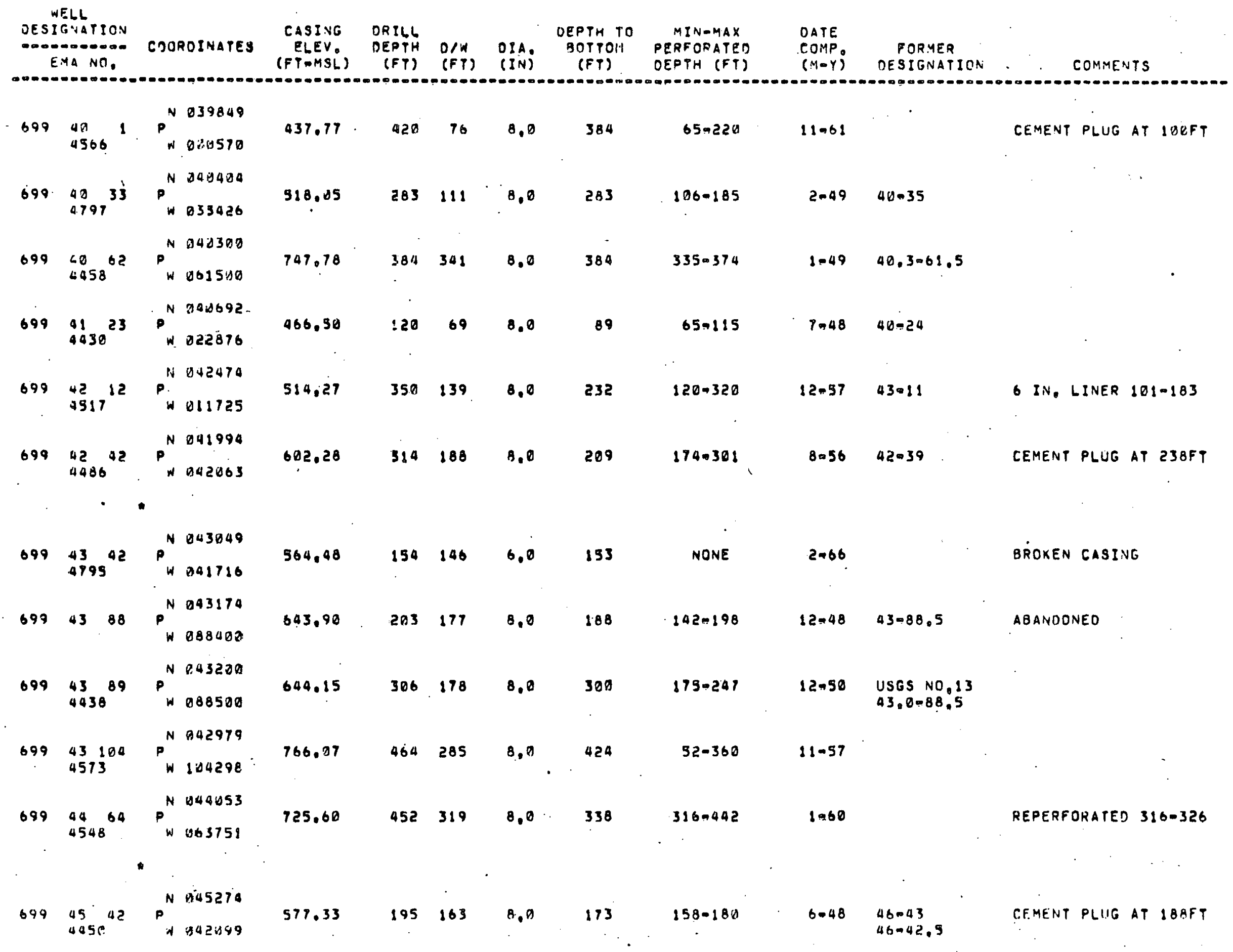


WELL

OESITINATION

hemionation

EMA NO.

COOROINATES

CASING DRILL

DEPTH

O/n. OIA. DEPTH TO

MINTMAX

PERFORATED OEPTH (FP)

DATE (FTOMSL)

(FT) (FF) (IN)

$\begin{array}{lllll}725.46 & 368 \quad 278 & 8.0 & 320\end{array}$

$274-366$

6048

$45-70$

$690 \operatorname{la}_{4049} 69 P^{N 045003}$

. 368.979

sen

$5-69.5$

699 N046093 $4479^{21}+020629$

522.22

$337 \quad 13$

$197 \quad 68$

B. 1

$133-220$

$9-55$

$45-20$

$\begin{array}{ll}6994739: 9^{N} 846860 \\ 4478 & =254659\end{array}$

$3994746 p^{N 047039}$ $4564^{46} P 045994$

980.19

$297 \quad 176$

0.0205

583.45

167 DRY

8.0

167

NONE

10.59

$4.8-51$

$235-277$

7.48

$47.5 \cdot 60.5$

$699 \quad 46 \quad 18$

424.98

0. 0

SEE HAN-24

$699 \quad 48 \quad 49$

5000

O. D ARHOOCI

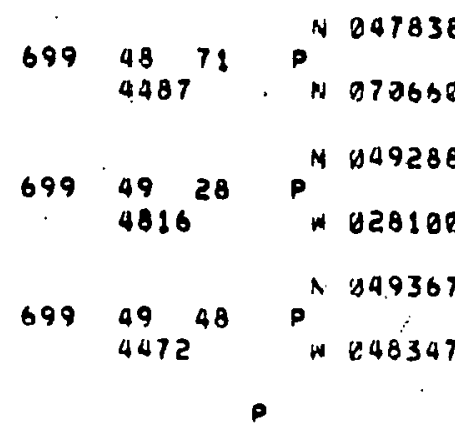

$6994955 p^{N 0440805}$ $4562^{55} P 059426$

68.8 .15

$305 \quad 244$

$0.0 \cdot 287$

2390302

$9 \cdot 56$

$535.91 \quad 179 \quad 143 \quad 6.0 \quad 178$

6-7:

SCREEN $167-177$

$\begin{array}{llllll}551.92 & 173 \quad 119 & 3,0 & 137 & \end{array}$

4.55

$50 \cdot 48.5$

$551.92 \quad 1165 \quad 145$

$4,0 \quad i 165$

NONE

$0-0$

$930.14 \cdot 149 \cdot 127$

8.8 148

$125-135$

$7-61$

\#39 SCREEN 124-139

REPERF ORATED 75-87FT CLEANEO TO B8FT

ASANDONED 


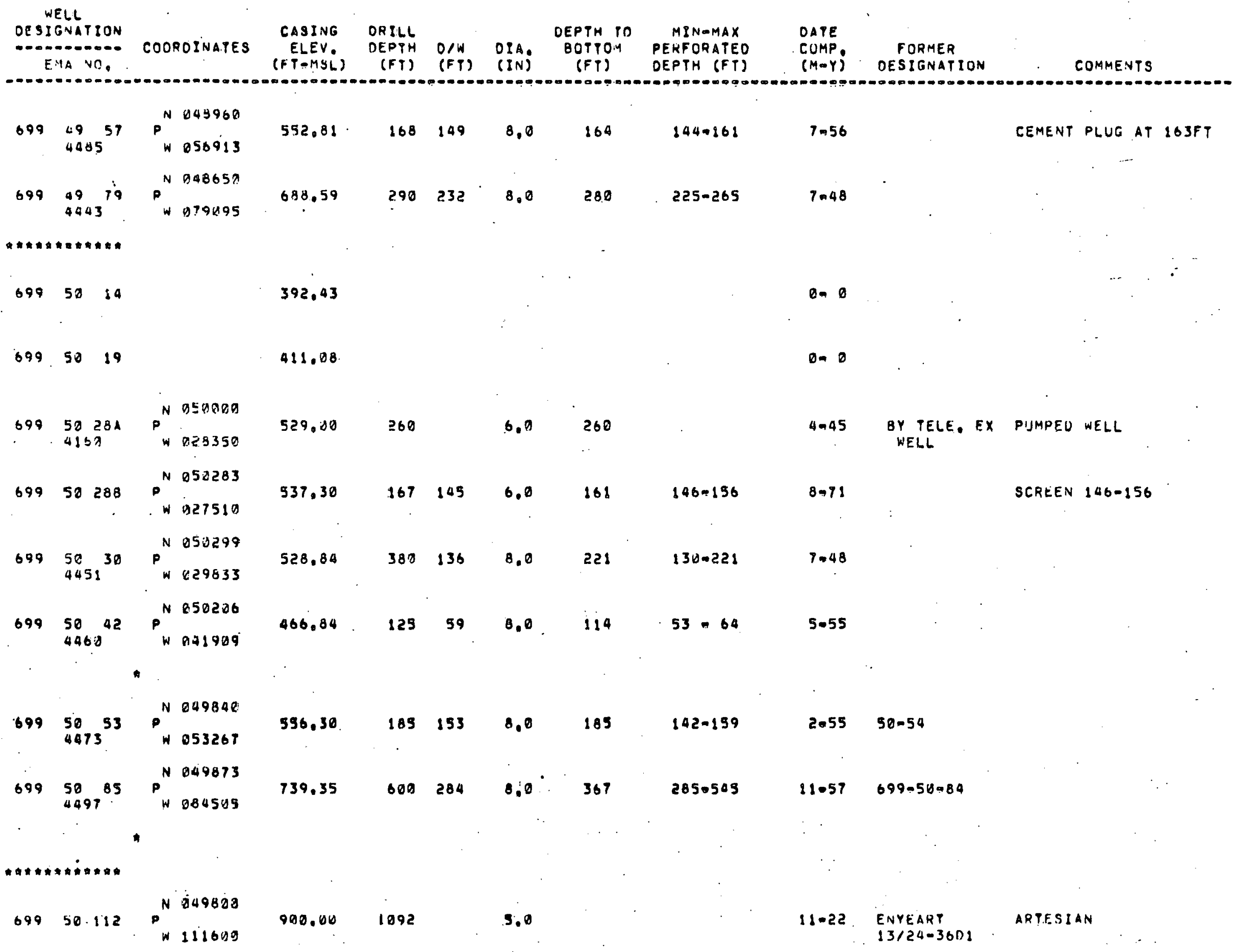




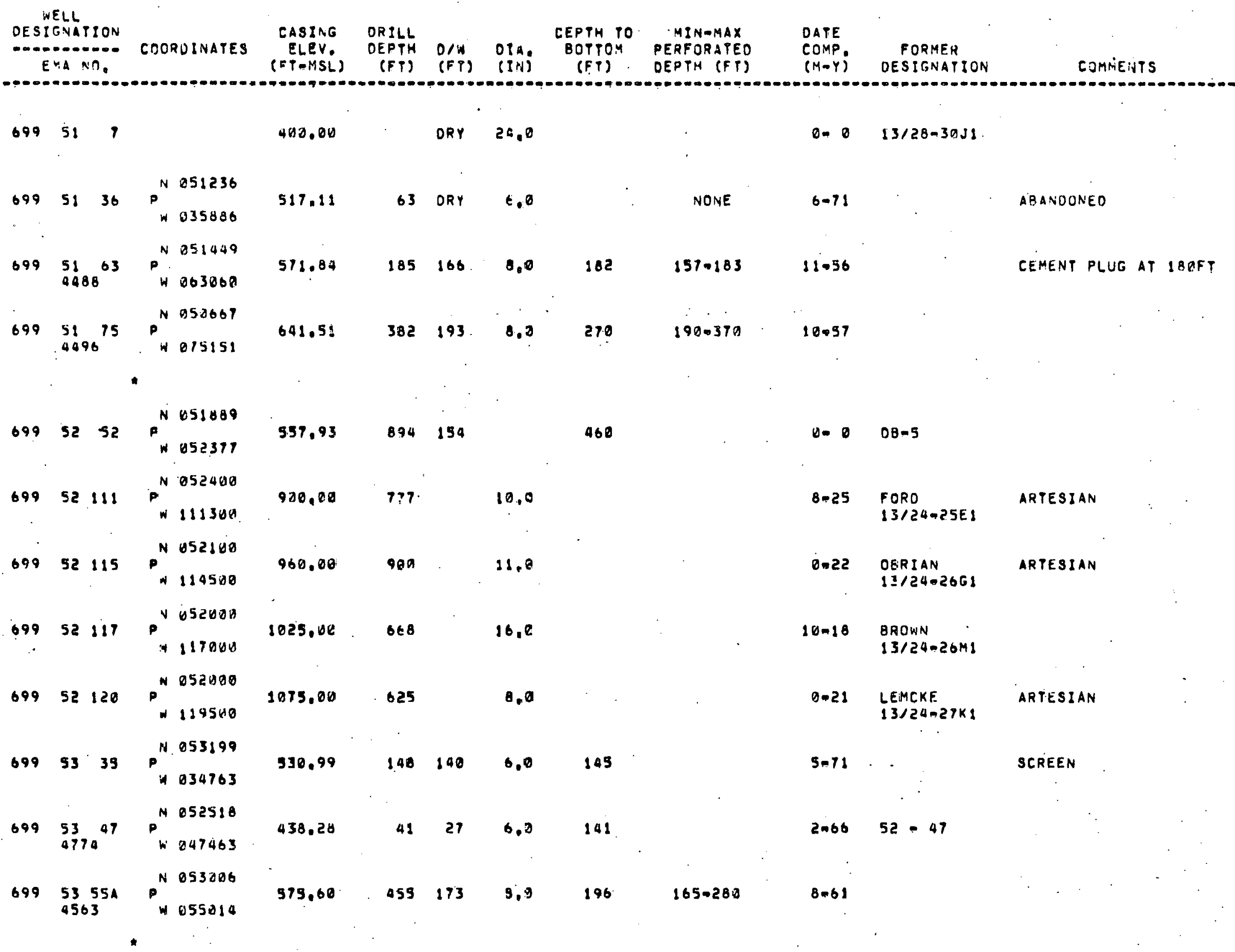


WELL

DESITNATION

EMA. NO.

COOROINATES

CASING DRILL

ELEV. OEPTH D/H OIA, BOTYOA PERFORATED

DEPTH (FT)

OATE

COMP FORMER

(M-Y) DESIGNATION

COMPENTS

\begin{tabular}{|c|c|c|c|c|c|c|c|c|c|c|c|c|c|}
\hline 699 & 535 & 558 & $P_{W}^{N}$ & $\begin{array}{l}052979 \\
055430\end{array}$ & 569.90 & $=52$ & & . & & . & $5-75$ & . & \\
\hline 699 & 535 & $55 \mathrm{C}$ & $P^{N}$ & $\begin{array}{l}052916 \\
055215\end{array}$ & 564.82 & & & & & & D. & & \\
\hline 699 & $\begin{array}{l}5.31 \\
6772\end{array}$ & $\int^{103}$ & $D^{N}$ & $\begin{array}{l}052729 \\
103420\end{array}$ & 837.28 & $19: 0$ & & 8.0 & 1110 & $165=280$ & $4-27$ & MCGEE WELL & ARTESIAN. \\
\hline 699 & 50 & 34 & $p^{N}$ & $\begin{array}{l}05+185 \\
0.30075\end{array}$ & 590.24 & 166 & 145 & 6.0 & 164 & . & 8.71 & & IO FT. SCREEN \\
\hline 699 & $\begin{array}{l}54 \\
4970\end{array}$ & 37 & $P^{N}$ & $\begin{array}{l}554140 \\
936806\end{array}$ & 534.17 & 970 & 128 & 6,0 & 132 & & 8.23 & $\begin{array}{l}\text { HAYNES STOCK } \\
\text { WELL }\end{array}$ & $\begin{array}{l}6,4,5,3,5 \text { IN CASINGS } \\
\text { CLEANED TO } 923 \mathrm{FT}\end{array}$ \\
\hline 699 . & $\begin{array}{l}50 \\
9432\end{array}$ & $2^{42}$ & $P$ & $\begin{array}{l}054390 \\
042431\end{array}$ & 511.49 & 210 & 112 & 8.0 & 182 & $100-200$ & $6-48$ & $54.5-42.5$ & CEMENT PLUG AT ISZFT \\
\hline 699 & $\begin{array}{l}54 \\
4811\end{array}$ & $1^{45}$ & $P^{N}$ & $\begin{array}{l}054203 \\
044536\end{array}$ & 494.25 & 105 & 98 & 6.0 & 105 & & 6071 & & SCREEN 95-105 \\
\hline 698 & $\begin{array}{l}54 \\
9459\end{array}$ & $9^{57}$ & $P^{N}$ & $\begin{array}{l}0.54311 \\
0 \sin 639\end{array}$ & 577.78 & 199 & 172 & 8,0 & 193 & 159.188 & 6.59 & $55-55$ & CLEANED TO $190 \mathrm{FT}$. \\
\hline 699 & $\begin{array}{l}55 \\
4731\end{array}$ & $2 !$ & $P_{W}^{N}$ & $\begin{array}{l}054020 \\
228449\end{array}$ & 395.96 & 48 & 34 & 42,0 & 35 & & $0=0$ & REF.2 NO. 18 & DUG WELL \\
\hline 699 & 55 & 48 & $P^{N}$ & $\begin{array}{l}055331 \\
940435\end{array}$ & 543.13 & $: 45$ & 136 & 6,0 & 140 & & 8.71 & - & SCREEN \\
\hline 699 & 59 & 44 & $p^{N}$ & $\begin{array}{l}055462 \\
043677\end{array}$ & 519.67 & 160 & 126 & 6,0 & 152 & $100-1.50$ & 0.71 & & SCREEN $14 Q-150$ \\
\hline
\end{tabular}




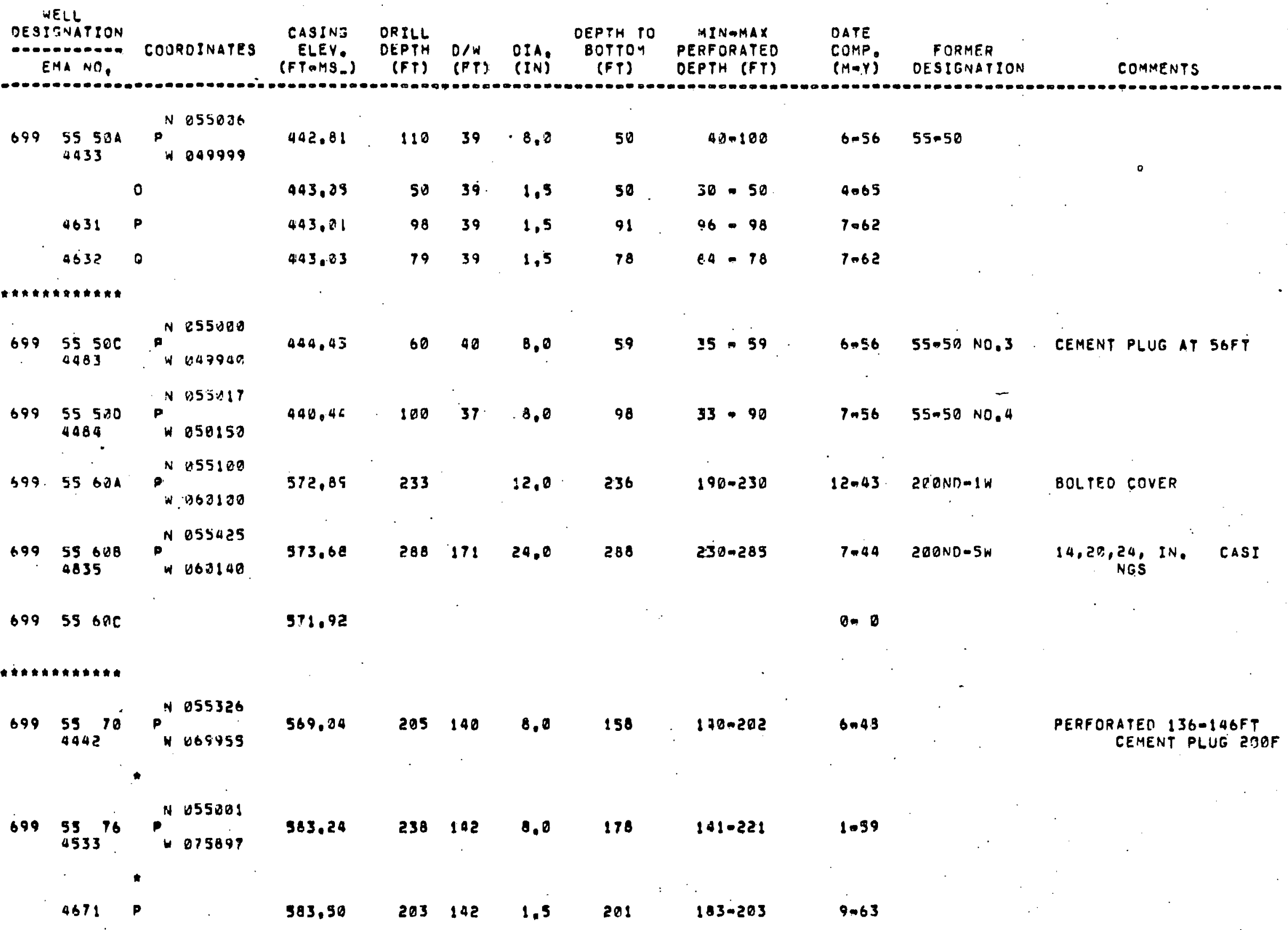


WELL

DESITHATION

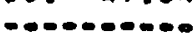

EMA NO.

COORDINATES

CASING

ELEV ORILL (FY:MSL)

DEPYH

DEPTH YO
D/W OIA. BOTTOY

MIN-MAX
PERFORATEO PERFORATEO
DEPTH (FT)

DATE

COMP.

$(M=Y)$

FORMER

DESIGNATION

COMMENTS

$\begin{array}{lll}699 & 5589 & P^{N} 054569 \\ 4453 & N 089542\end{array}$

0

$\begin{array}{llll}235 & 165 & 0.0 & 194\end{array}$

$170=222$

$11=48$

$.55-88.5$

$017.69 \quad 190 \quad 169 \quad 1,5^{\circ} 191$

$617.69 .220 \quad 165 \quad .1 .5 \quad 220$

$160-190$

$4-65$

N 055000

777.05

$530310 \quad 8.0 \quad 338$

$200-228$

9.63

4934

W 094950

777.35

$\begin{array}{llll}519 & 348 & 1.5 & 519\end{array}$

$325=515$

$\operatorname{1n} 59$

797.35

$a \in 5 \quad 332 \quad 1.9 \quad 434$

$499-519$

6.64

0

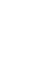

$6995626 p^{N 055801}$

พ. 225736

409.84

25 ORY

6.0

$8-71$

AGANDUNEO

$69956430^{N 053261}$

54.0 .42

$155 \quad 233$

6.0

154

$138-155$

$6-71$

SCREEN $138-155$

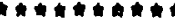

$6995725 \quad$ N 05t.755

. $25 \quad P$ W 025477

414,57

$150^{\circ} 5$

6.0

64

$52 \cdot 80$

$7=71$

DEEPENEO 1-74, SCREEN REMOVED

เ99 57 N 057100

$6995_{4462}^{59} P^{N 020132}$
6853
32.0

68

50.65

10.44

PISTOL RANGE

CASING TO TOP OF PIT
$5.92 \mathrm{FT}$

$6995783 \quad P^{N .651020}$

577.9

$359 \quad 147 \quad 0.0: 179$

$150-348$

22060

578.22

$170 \cdot 147 \quad 1.5 \quad 170$

$240-170$

$4-65$

578.22

$355 \quad 847.1,5 \quad 355$

$335-355$

6.64

578.22

$292 \quad 149 \quad 1.5 \quad 292$

$270-290$

$6-64$

R

978.22

$\begin{array}{llll}232 & 149 & 1.5 & 232\end{array}$

210.233

$6-64$ 
HELL.

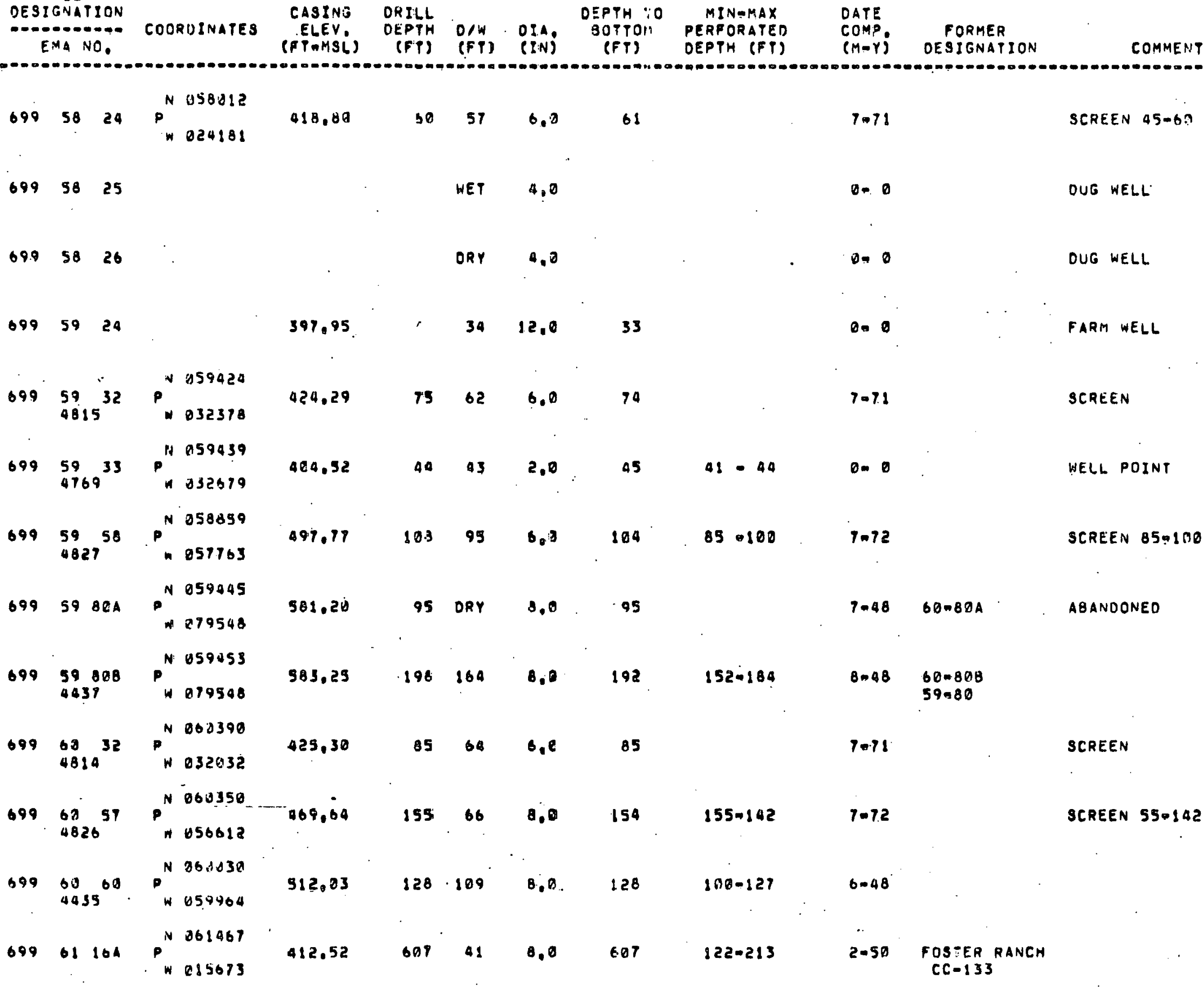




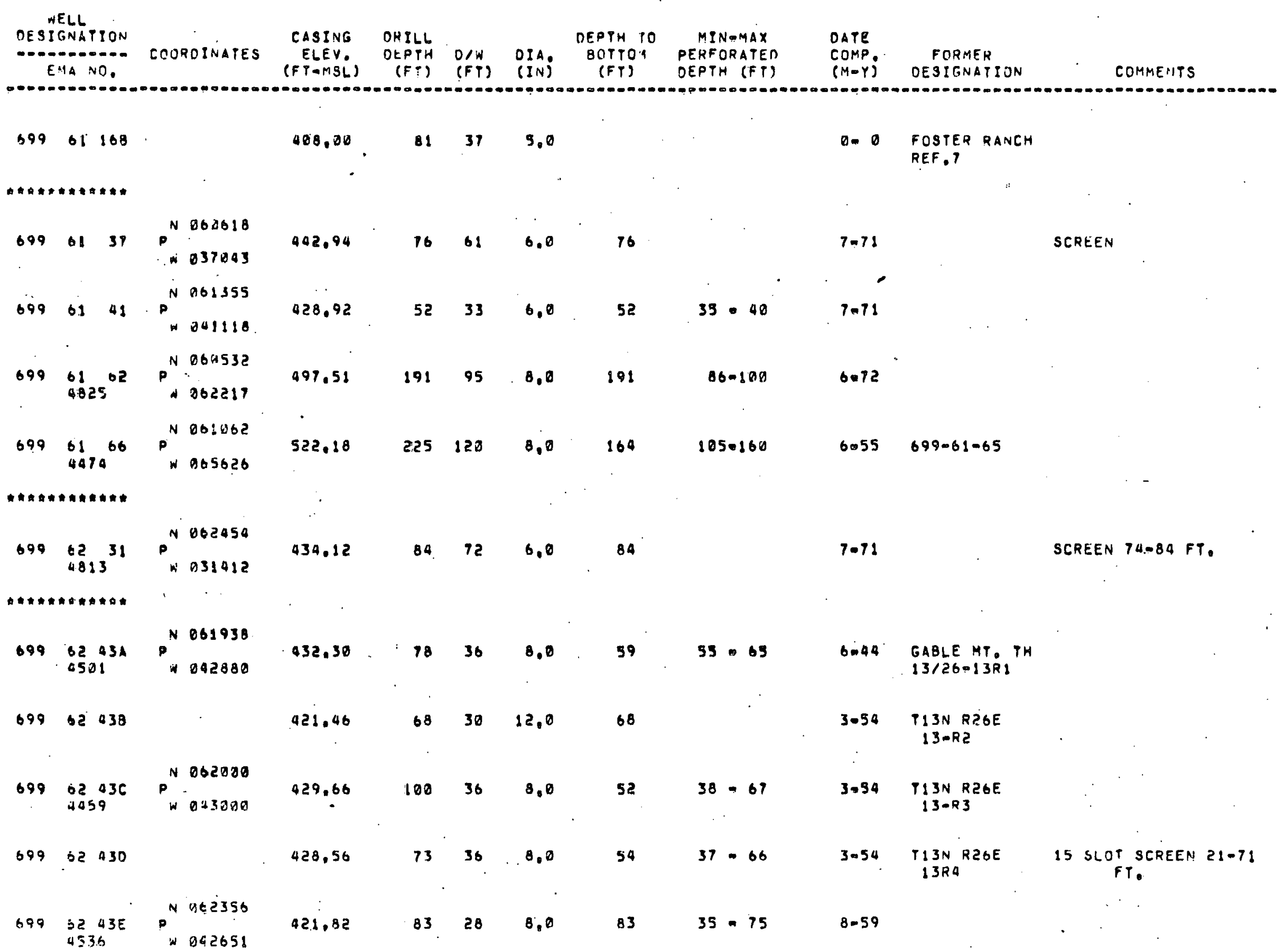


WELL

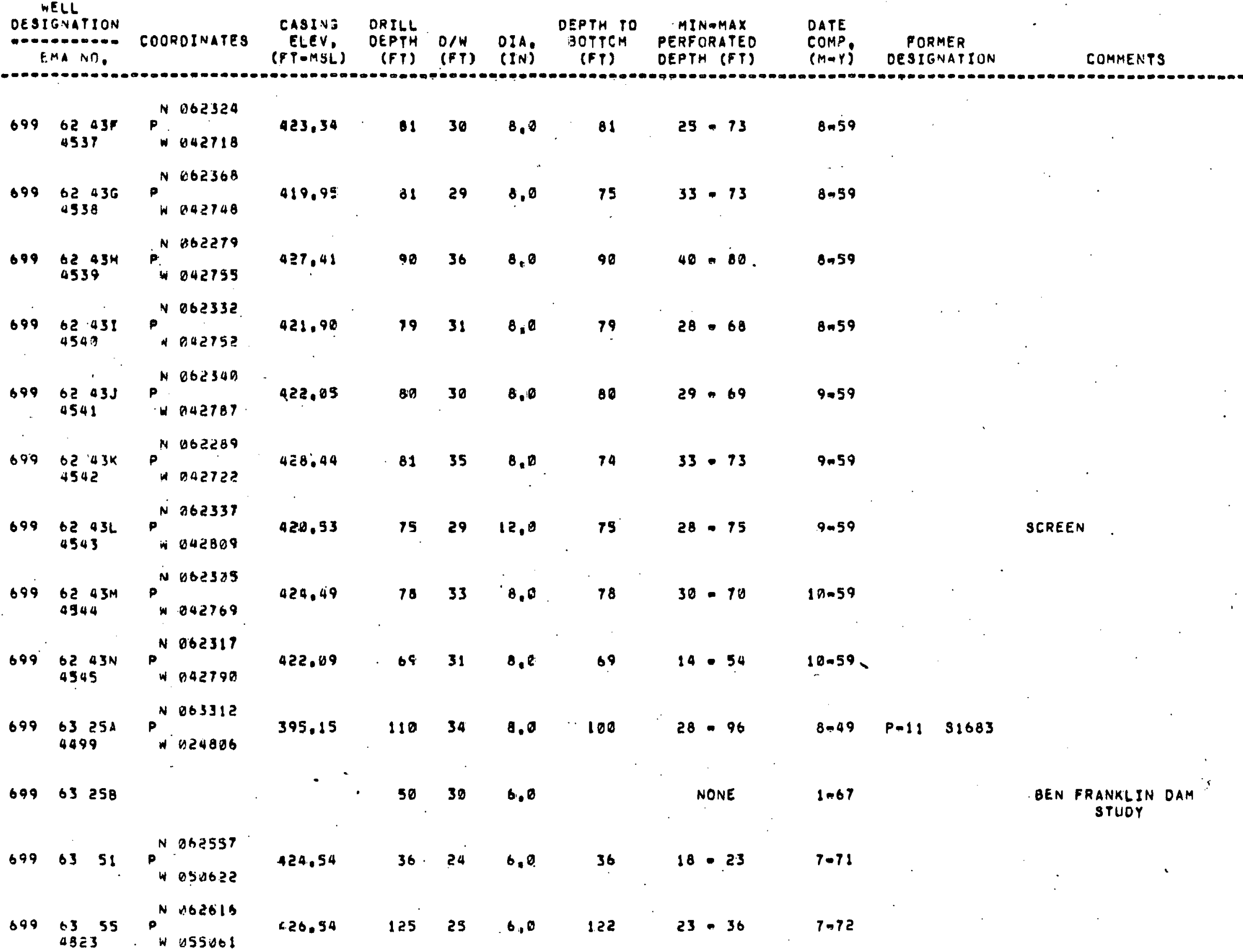




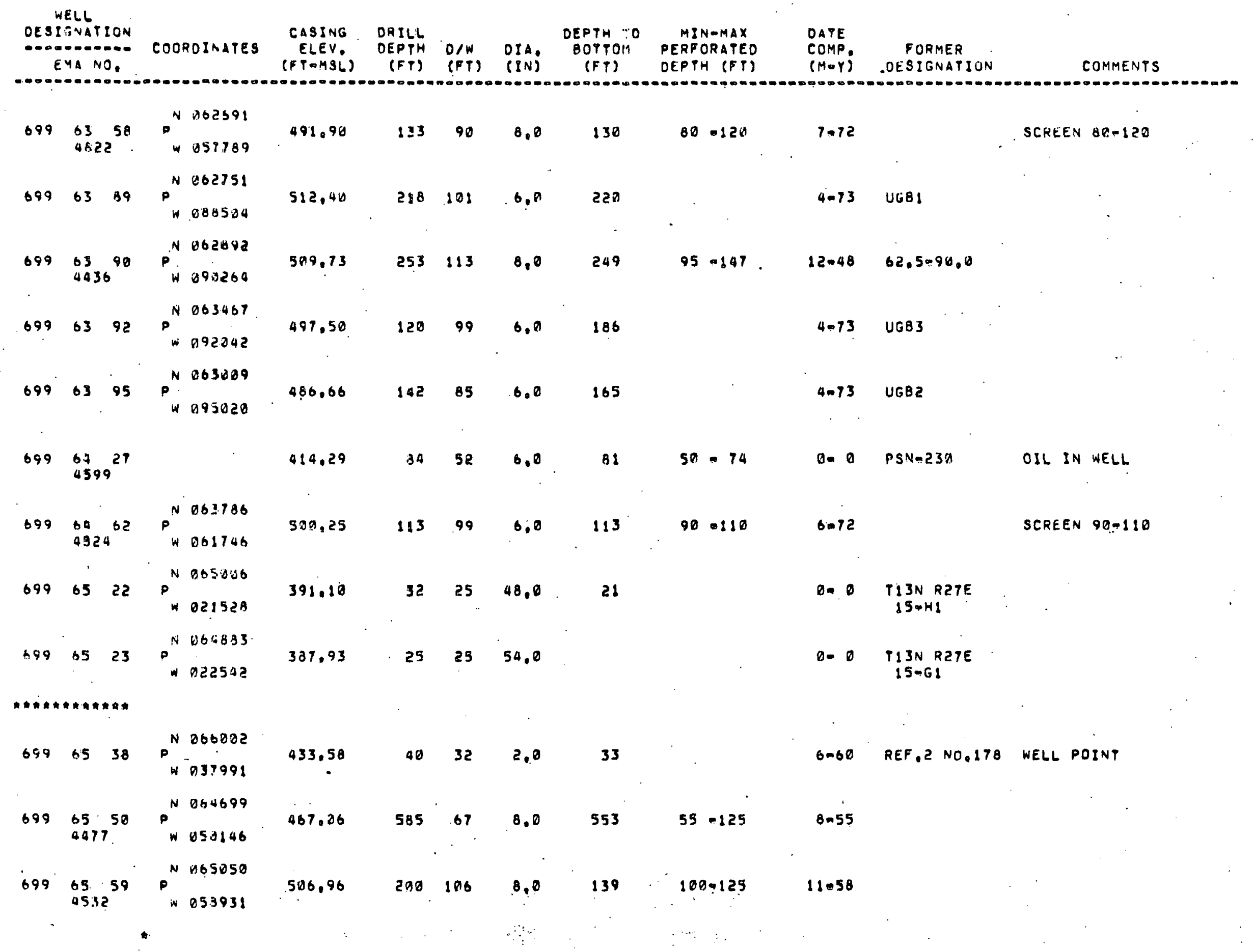




\begin{tabular}{|c|c|c|c|c|c|c|c|c|c|c|c|c|c|}
\hline$\frac{\text { OESI }}{-\quad E}$ & $\begin{array}{l}\text { ELL } \\
\text { CNATION } \\
\text { MA NO. }\end{array}$ & COOR & ROIVATES & $\begin{array}{c}\text { CASING } \\
\text { ELEV } \\
\text { (PYOMSL) }\end{array}$ & $\begin{array}{l}\text { DRILL } \\
\text { DEPTH } \\
\text { (F?) }\end{array}$ & (FT) & $\begin{array}{l}2\left[A_{1}\right. \\
\text { IIN] }\end{array}$ & $\begin{array}{l}\text { DEPTH } 10 \\
\text { EOTPO:A } \\
\text { (FT) }\end{array}$ & $\begin{array}{l}\text { MIN-MAX } \\
\text { PERFORATED } \\
\text { OEPYH (FT) }\end{array}$ & $\begin{array}{l}\text { DATE } \\
\text { COMP, } \\
\text { (MAY) }\end{array}$ & $\begin{array}{l}\text { FORMER } \\
\text { DESIIGNATION }\end{array}$ & & COMMENTS \\
\hline 699 & $\begin{array}{l}65 \\
4403^{72}\end{array}$ & $P^{N}$ & $\begin{array}{l}064452 \\
072156\end{array}$ & 570,28 & 216 & 142 & $22 ; 0$ & 214 & $137 \cdot 157$ & $\theta=0$ & $\begin{array}{l}\text { REF, } \\
\text { RANCH } 13\end{array}$ & & \\
\hline 699 & ${ }_{4773^{83}}^{65}$ & $p^{v}$ & $\begin{array}{l}064944 \\
092961\end{array}$ & 485,63 & 121 & 88 & 6.2 & 120 & $60-120$ & 4.67 & & & $\cdot$ \\
\hline 399 & 6585 & $i^{N}$ & $\begin{array}{l}804783 \\
095301\end{array}$ & 452,20 & 142 & 54 & 6.0 & 104 & . & $4-73$ & UG84 & & \\
\hline 699 & 651141 & $P^{N}$ & $\begin{array}{l}064770 \\
113930\end{array}$ & 462.10 & 185 & & $B, D$ & 185 & & $12-49$ & $\begin{array}{l}\text { BONNE, POWER } \\
\text { AOH, NO,Z }\end{array}$ & & \\
\hline 699 & 651148 & $p^{N}$ & $\begin{array}{l}065300 \\
119450\end{array}$ & 4.61 .40 & 122 & & 10.0 & 102 & & 0.52 & $\begin{array}{l}\text { BONNE, POWER } \\
\text { AOM, NO, } 1\end{array}$ & & . \\
\hline 699 & ${ }_{4547^{63}}^{23}$ & $P^{N}$ & $\begin{array}{l}005994 \\
022979\end{array}$ & 389.81 & 120 & 29 & 8,0 & 59 & $20 \cdot 96$ & 10.61 & & & \\
\hline & & 0 & & 389.27 & 58 & 29 & $\therefore, 5$ & 50 & $30-50$ & $5-65$ & & & \\
\hline & 4668 & $p$ & & 389.27 & 84 & 29 & 1,5 & 81 & 80.100 & 9063 & & & \\
\hline 699 & $\begin{array}{l}6638 \\
4586\end{array}$ & $P_{W}^{N}$ & $\begin{array}{l}060000 \\
038000\end{array}$ & 436.20 & 150 & 34 & 8,8 & 90 & $29-145$ & $18=62$ & . & & \\
\hline & & 0. & & 436.46 & 60 & 34 & 1.5 & 60 & & 5.65 & & & \\
\hline & 4659 & $p$ & & 236.46 & 150 & 49. & 1,5 & 125 & $130-150$ & 9.63 & & & \\
\hline & 4690 & A & & 436.46 & 110 & 49 & 1.5 & $: 10$ & $90-110$ & 9.63 & & & - \\
\hline 699 & $4812^{39}$ & $P_{W}^{N}$. & $\begin{array}{r}066099 \\
039459\end{array}$ & $\begin{array}{c}453.70 \\
?\end{array}$ & 90 & 47 & 6,2 & 90 & $38 \cdot 65$ & $7-71$ & - & . & . \\
\hline 699 & $\begin{array}{l}66 \\
4821\end{array}$ & $D^{N}$ & 457768 & 583.33 & 115 & 102 & 6,0 & 214 & $93-112$ & $7-72$ & . & SCREEN & $93-112$ \\
\hline 699 & $\begin{array}{l}96864 \\
4824^{64}\end{array}$ & $P^{N}$ & $\begin{array}{l}066483 \\
064249\end{array}$ & 505,92 & 120 & 106 & 6.0 & 120 & $96-116$ & $6-72$ & & SCREEN & $96-116$ \\
\hline
\end{tabular}




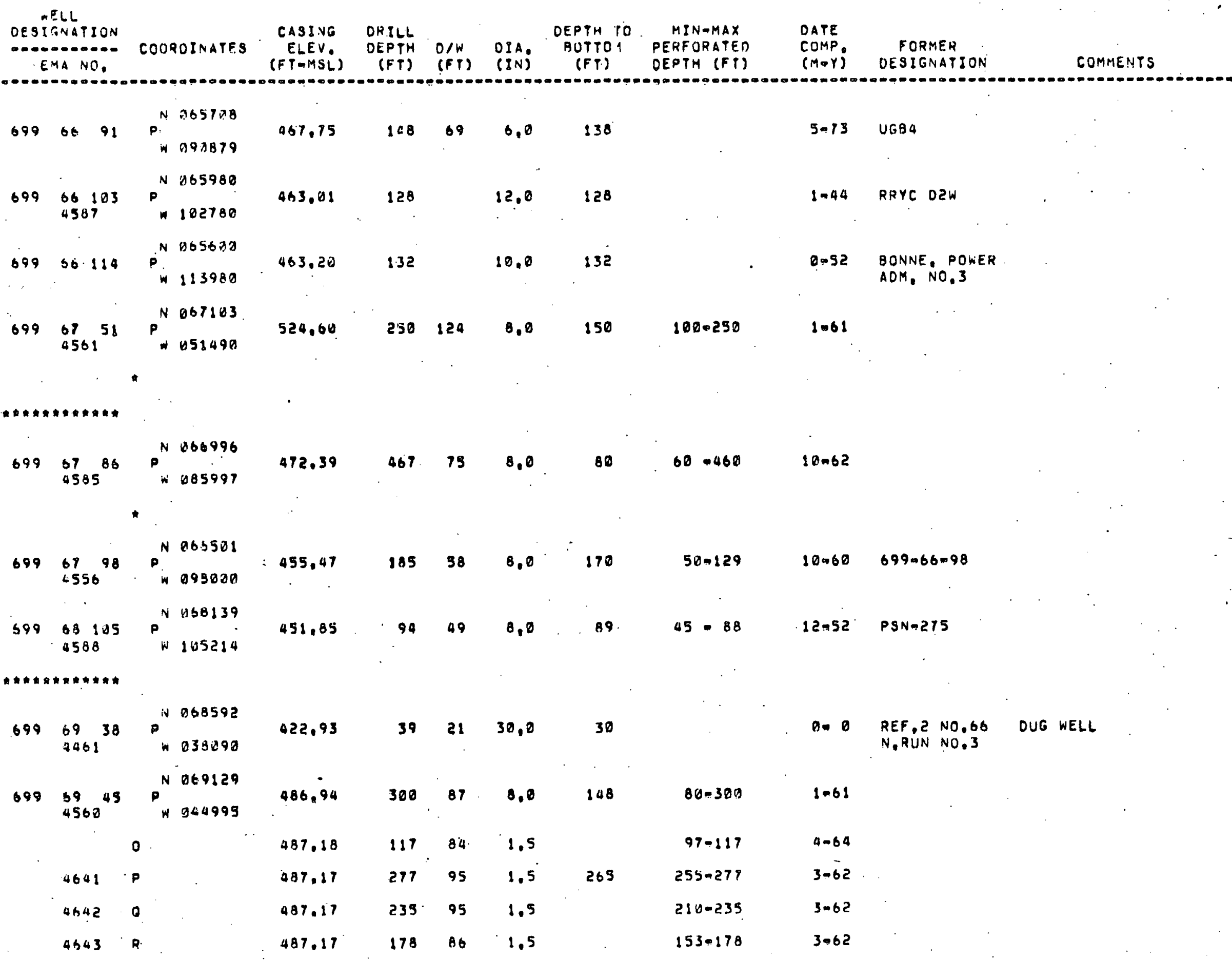




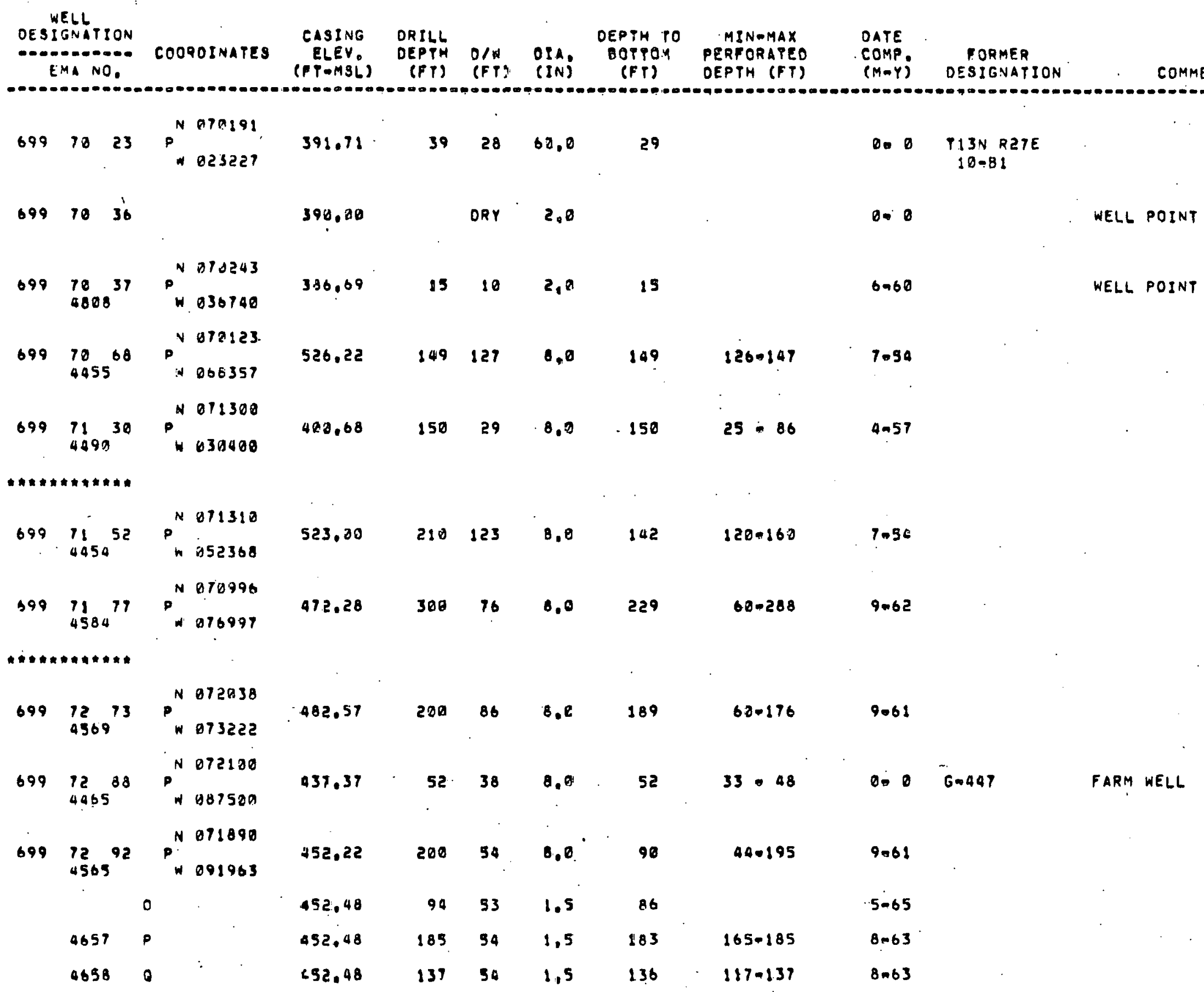




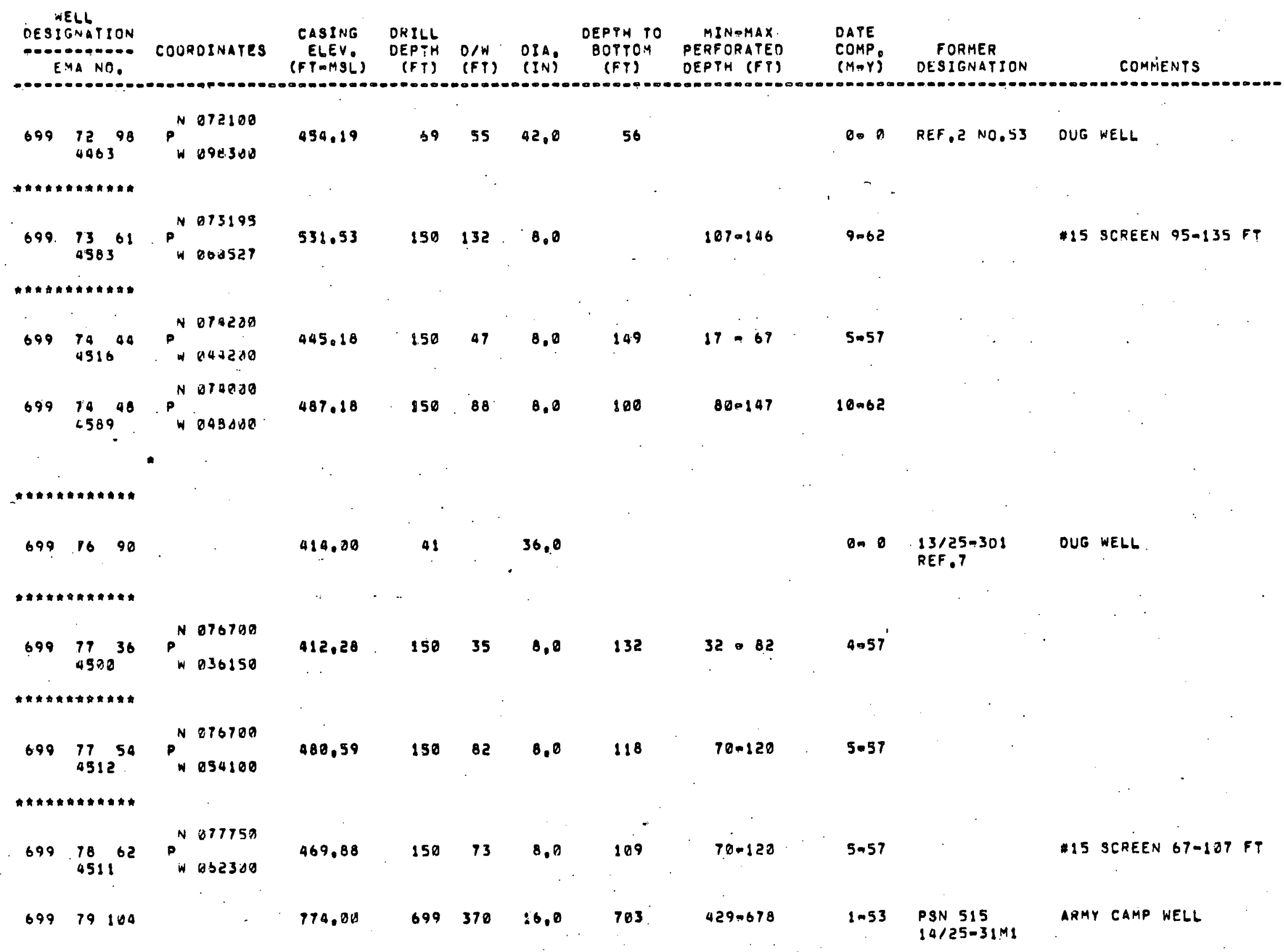


WELL

DESIGNATION

EMA ND.

COOROINATES

CASIN3 ORILL

DEPYH

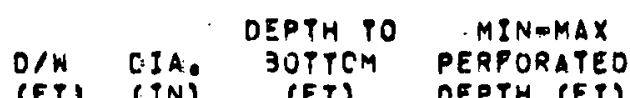

DEPTH (FT)

DATE

COMP.

PORMER

DESIGNATION

COMMENTS

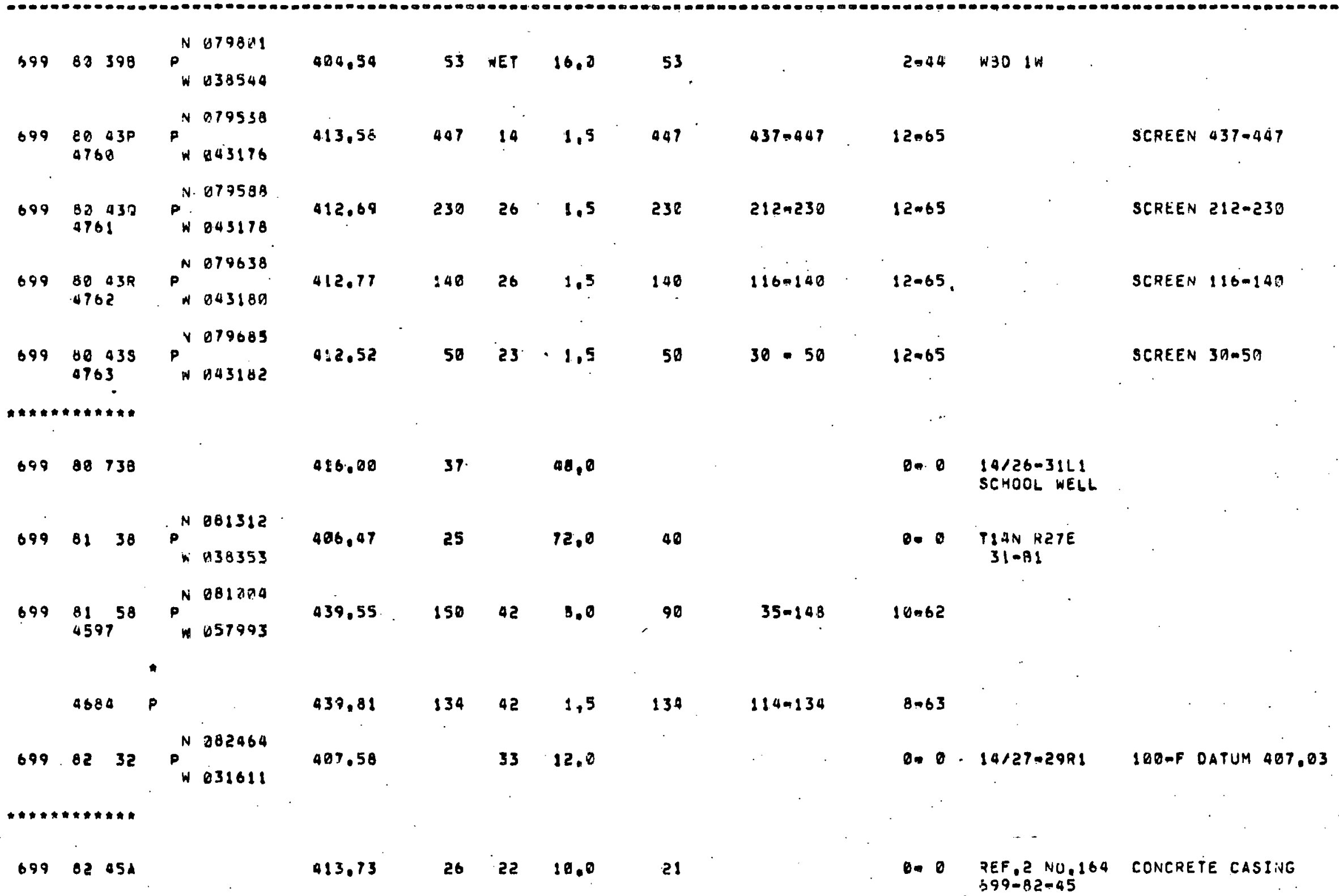




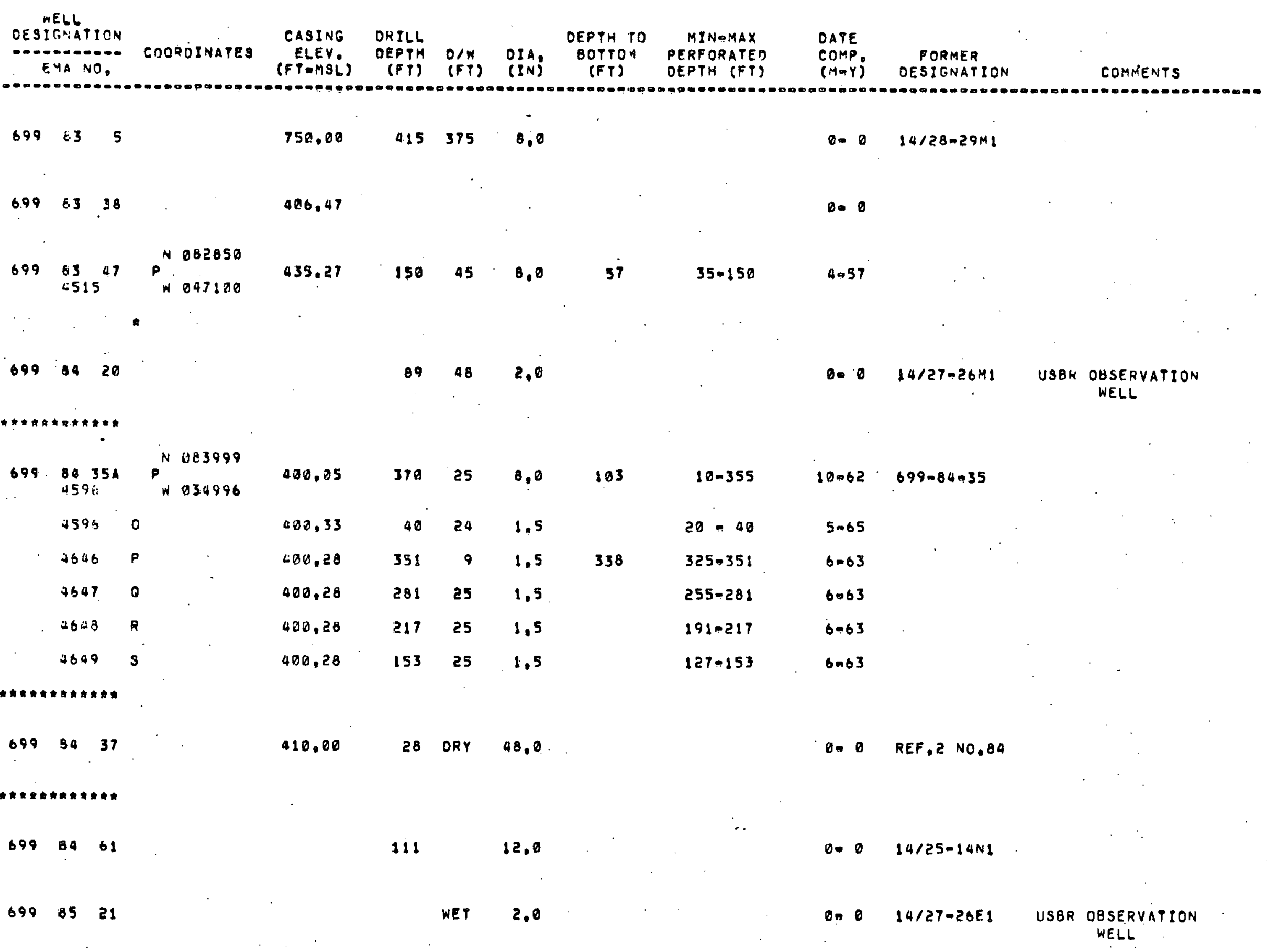


WELL

OESIGNATION

EMA NQ.

COOROINATES

CASING ORILL

OEPTH TO MINEMAX

PERFORATEO

DATE

COMP

FORMER

DESIGNATION

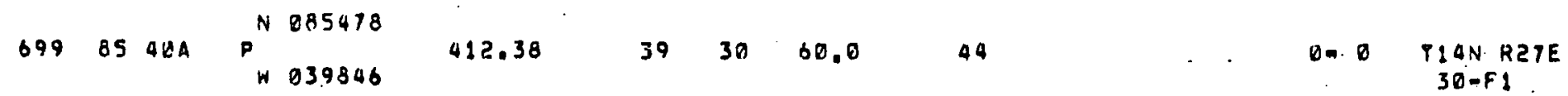

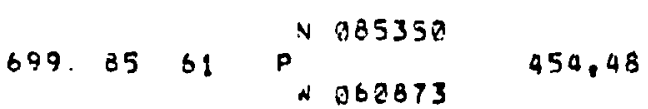

$699 \quad 86 \quad 351$

$699 \quad 86 \quad 358$

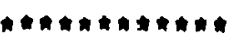

$699.86 \quad 42 \quad P_{N 042135}^{N} 485693 \quad 469.92 \quad 42 \quad 25 \quad 72,0 \quad 42$

$699 \int_{4570}^{60} P^{N} 085723$

$69986 \quad 95$

699872

822.09

500

8.0

$699 \quad 8724$

68 ORY

2,8

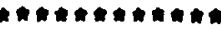

$699 \quad 87$ 921 N087187 4793 W

016.53

$3941 \cdot 12,2$

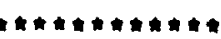

$6998755 P^{N 086707}$ 4792 . 055425

24.0

18.6
0.0

O- REF。2 NO,172 $699-86-35$

O. $0 \quad 699.86-35 A$

O. O TIAN RZTE DUG WELL

10.62

D- 14125-28EI NOT LOCATED

D. D MICHEL RANCH PUMPEO WELL 14/28-2981

Q. $14 / 27.2701$ USBR OBSERVATION WELL

O- D REF.? NO.119. DUG WELL $14 / 27-3002$

$6.69 \quad 100 \mathrm{~N} N 0.3$

$199-N-16$ 


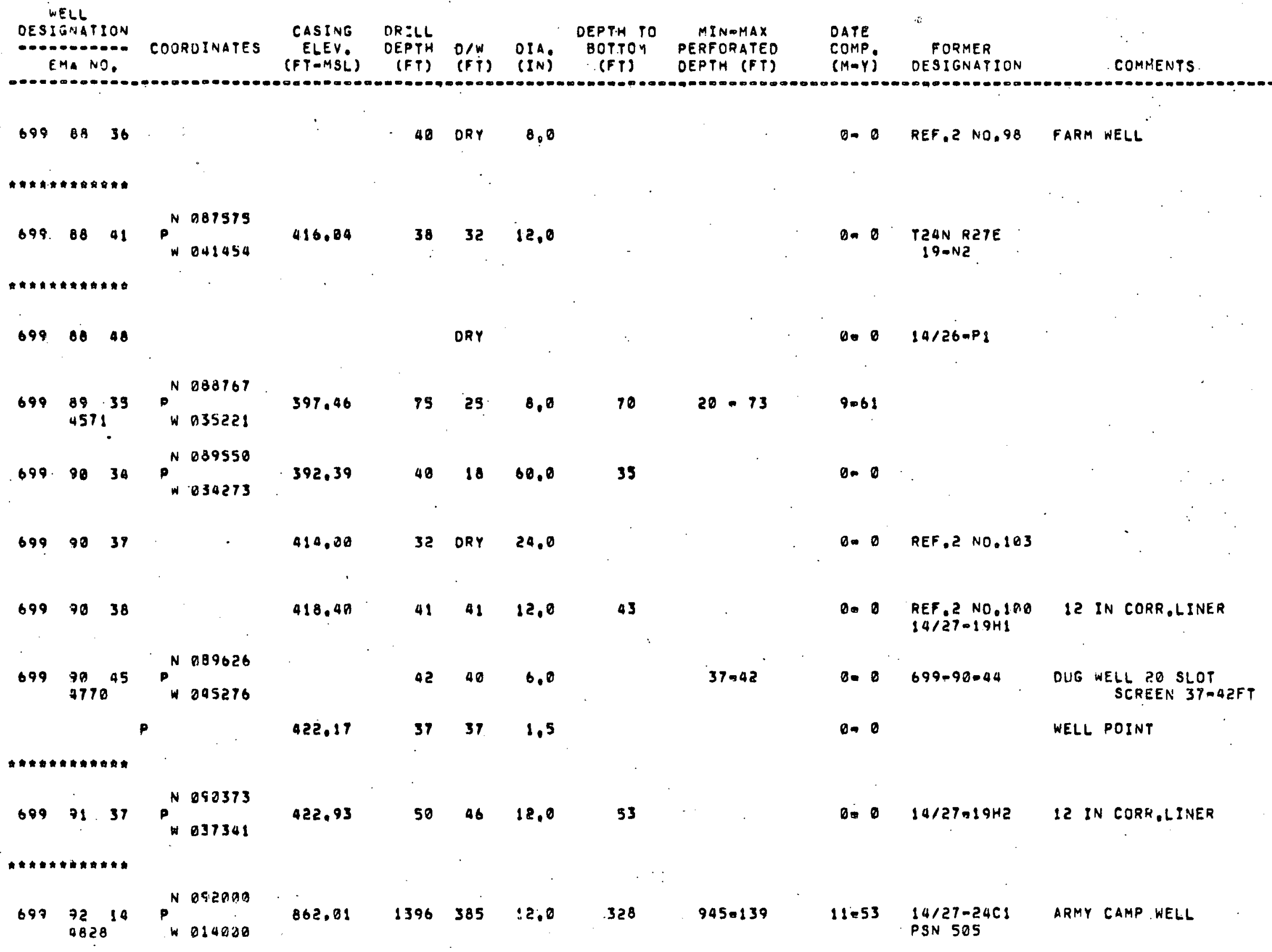


WELL

DESIGNATION

EMA NO.

COORDINATES

CASING

ORILL DEPTA PO

$M I N-M A X$

$\begin{array}{ccccc}\text { OEPTH D/W } & \text { D:AO BOTTOY PERFORATEO } \\ \text { (FT) (FT) (EY) (FT) OEPTH (FY) }\end{array}$

DATE

FT॰MSL)

COMP

FORMER

(M-Y) DESIGNATION

COMMENTS

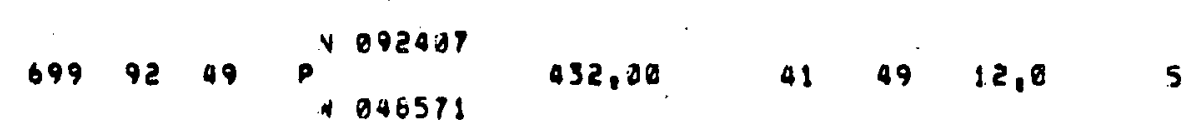

0. 14/25-23A1

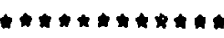

699.9393

637.81

520

$235 \quad 26.0$

515

$262-516$

$5-53$

$14.24-2181$

ARMY CAMP WELL

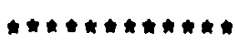

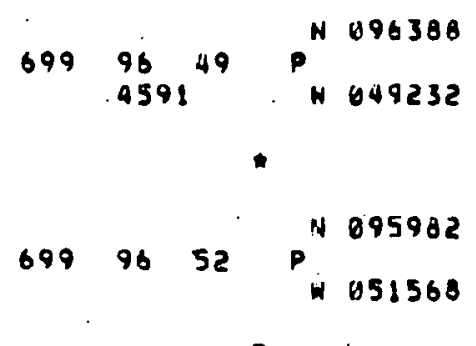

419.29

100

8.0

58

$28 \cdot 96$

$10=62 \quad H-15$

$p$

412.52

$30 \quad 12.0$

$30 \quad 1.5$

36

DUG WELL

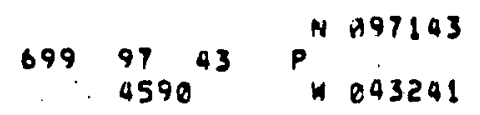

421.81

$100 \quad 43 \quad 8.0$

60

$25-97$

922.10

$6043 \quad 1,5$

6

$40 \cdot 60$

$70 \cdot 90$

$4693 P$

$\begin{array}{llll}699 & 97 \quad 47 \quad P^{M} 096735 \\ M & 047285\end{array}$

413.00

26 ORY 48.2026

49.2 .84

ORY $\quad 1.5$

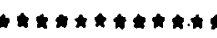

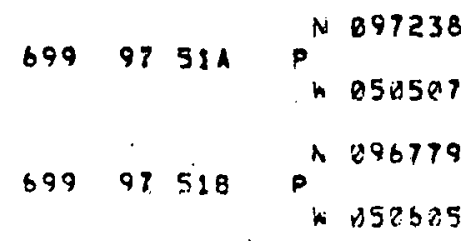

$\begin{array}{lllll}402.49 & 32 & 21 & 10.7 & 37\end{array}$

$496,50 \quad 28 \quad 22 \quad 12.0 \quad 31$

$\begin{array}{lllll}407.11 & 28 & 25 & 1.5 & 32\end{array}$
D. 0
0.65
$+$
$10-62$
8.63

D. REF, 2 NO.141 DUG WELL.

8. 0

WELL POINT
- - REF.2 NO.142 $97.50,97-51$

D. 0 $14,26-14 C 1$

FILLED AROUND 12 IN

$0 \cdot 0$

NELL POINT 


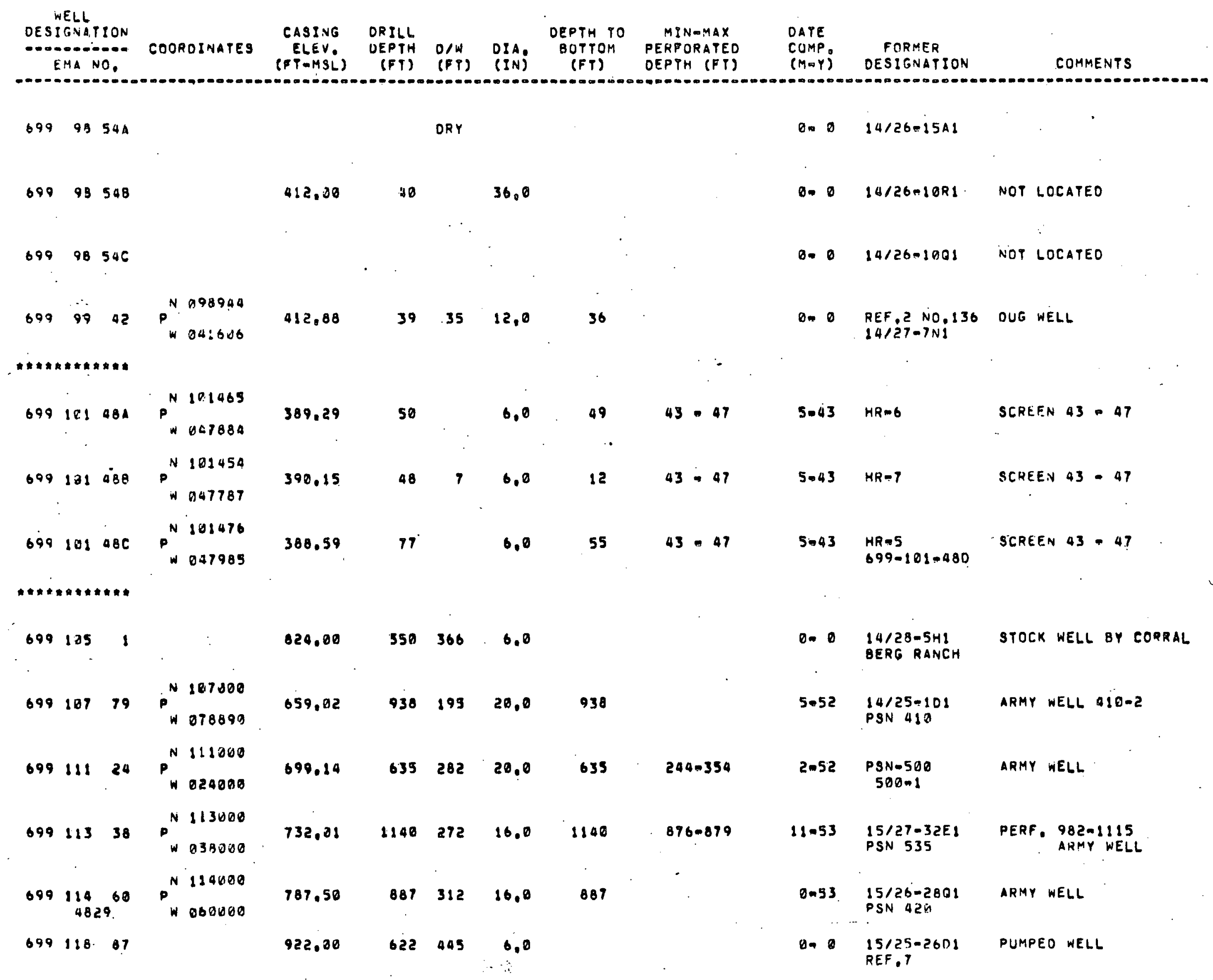


WELL.
DESTGNAT

OESIGNATION

EMA NO

COOROINATES

CASIVG ORILL

DEPTH TO MINGMAX

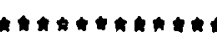

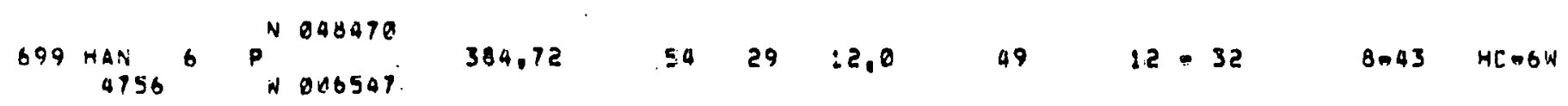

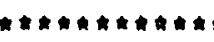

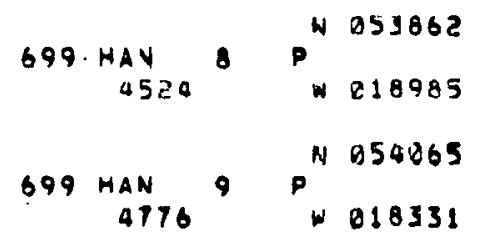

383.60

60

$22 \quad 24.0$

44

394.46

90

$26 \quad 16,0$

83

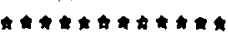

699 HAN $19 P^{N} 048825$

412.72

$8.252 \quad 20.0$

82

$55 \cdot 75$

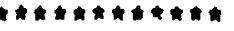

b99 HAN $24 \quad P^{N 047903}$

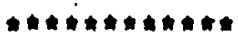

699 HAN $27 P^{N 049857}$ 1 18552

411.08

14

. N 049880

699 MAN. 28 P. O. O13917

392.43

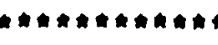

699 MAN 30

997.50

68

60.8

68

48.0 $\quad 52$

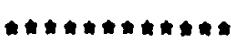

699 MAN 33

412.20

$\mathbf{3 3}$
$10.43 \quad H 0.8 W$

$12=4.3 \quad \mathrm{HO} 9 \mathrm{~W}$

PUMPED WELL

- D REF. 2 NO.30 NOT LOCATED 


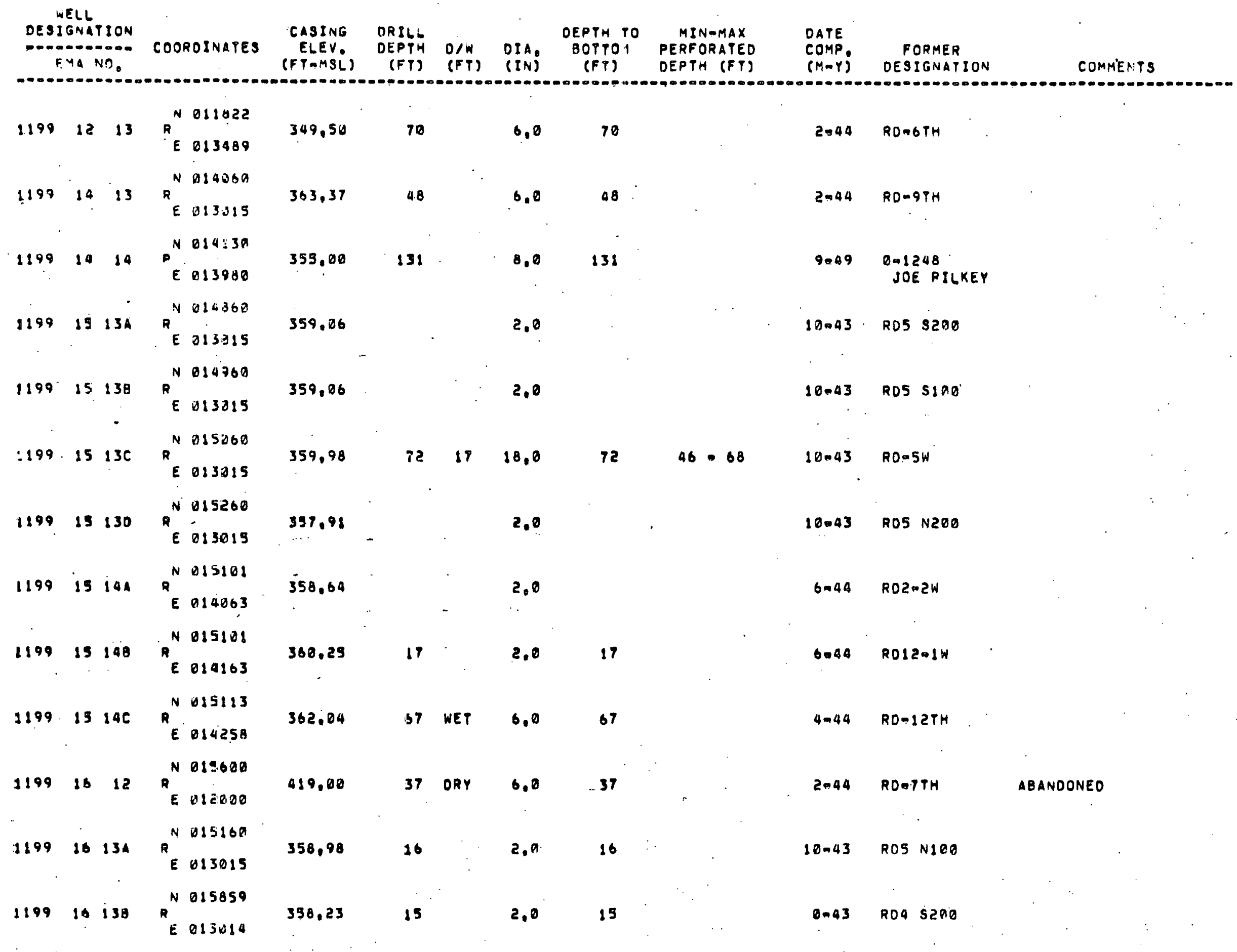




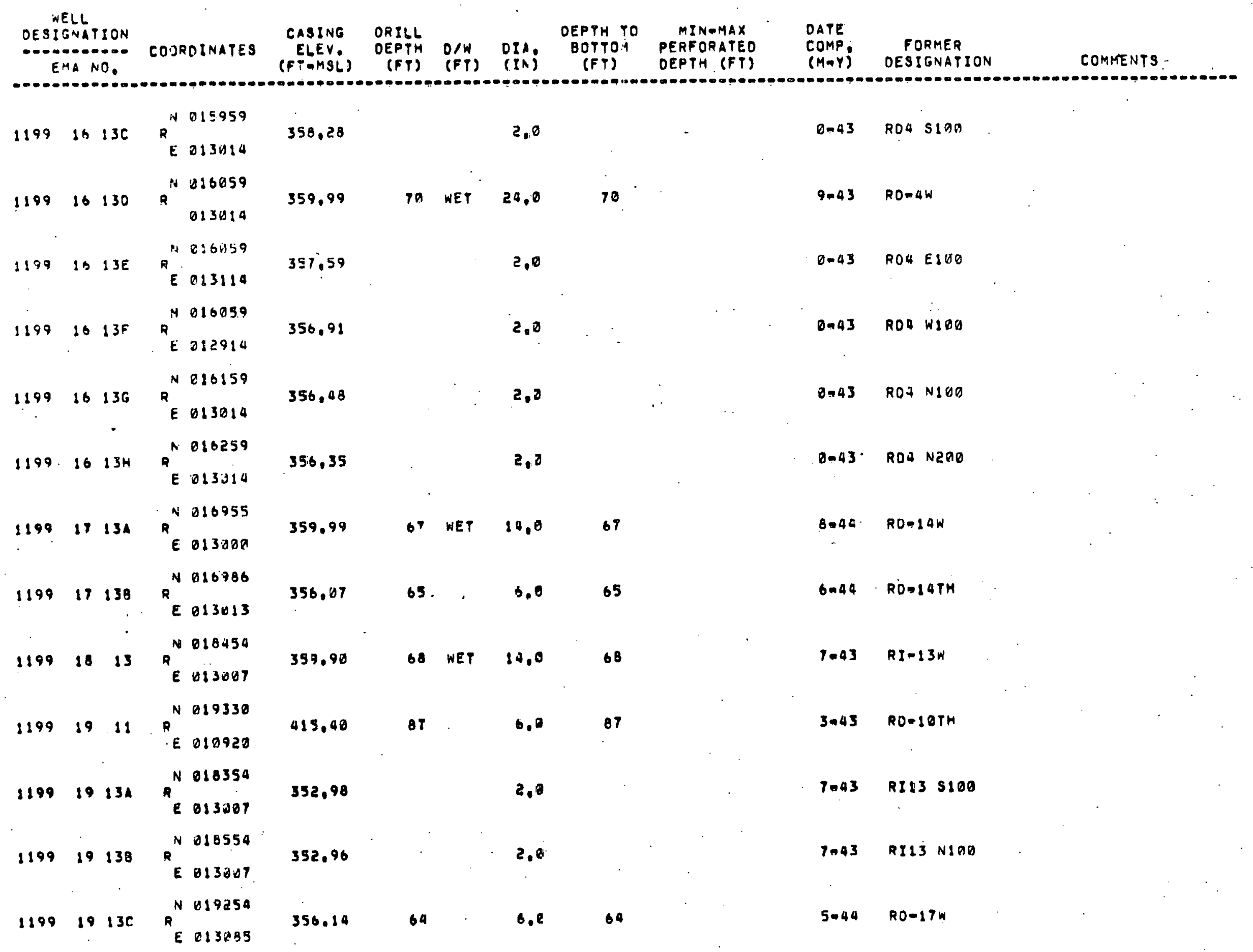




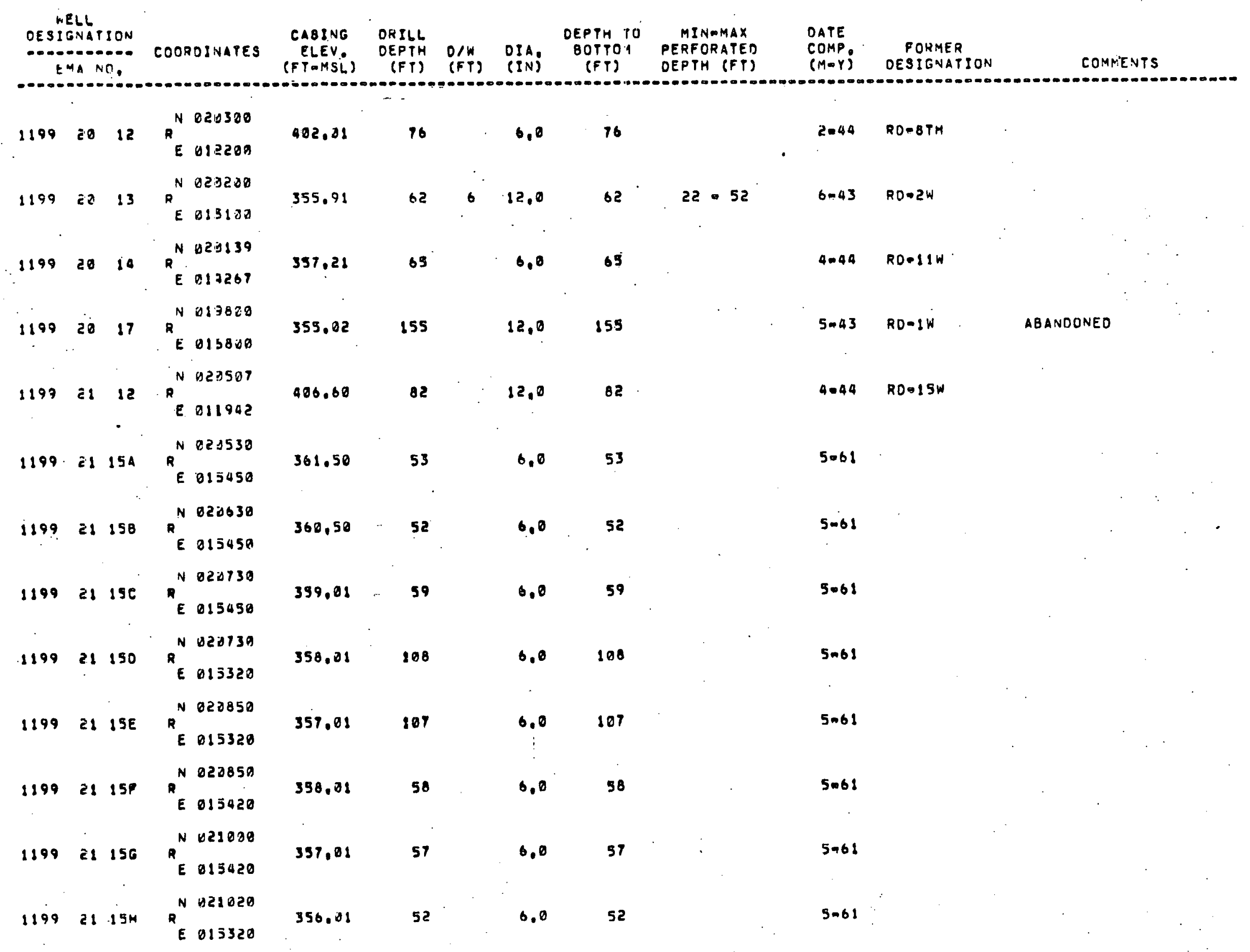




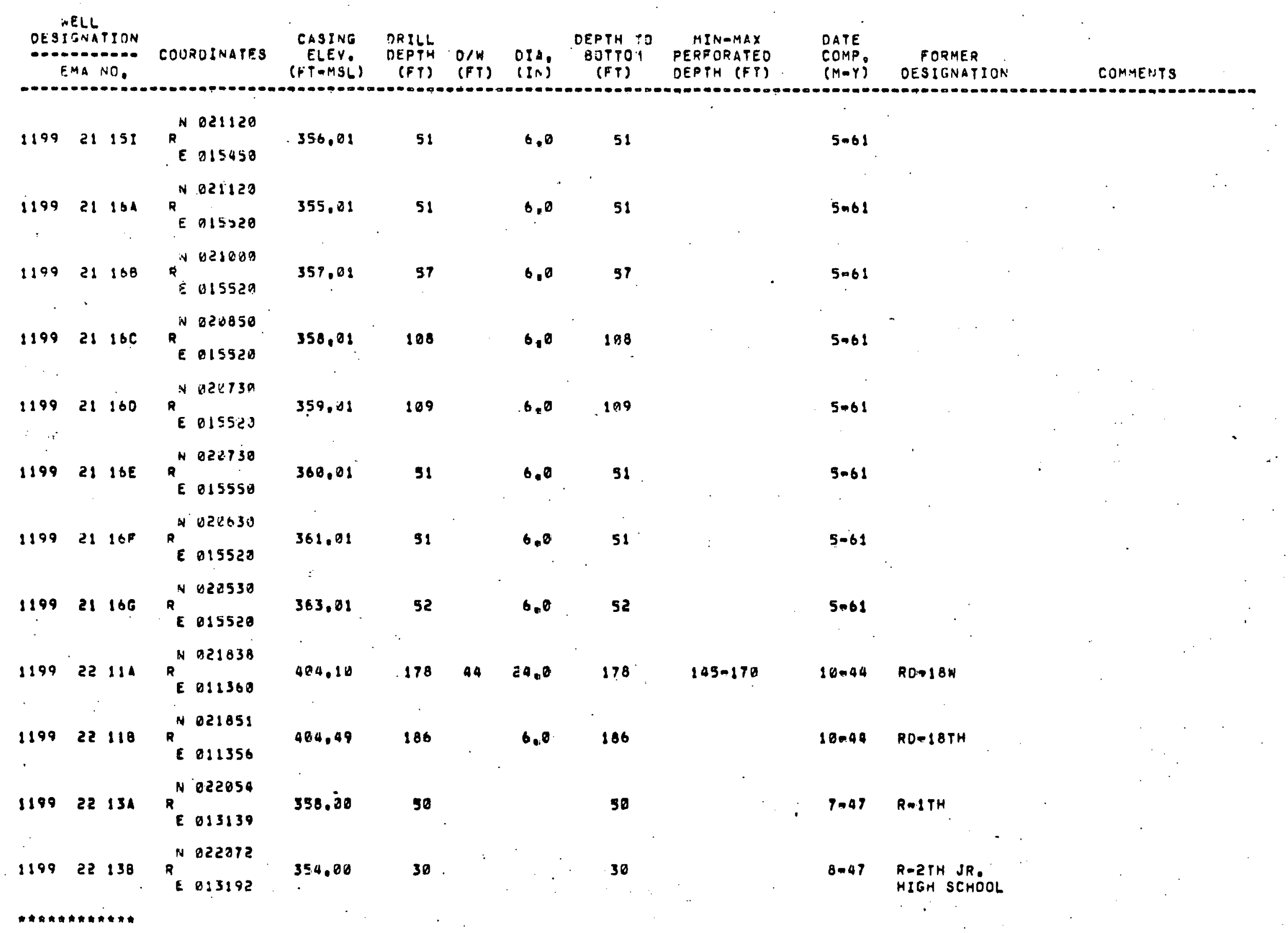




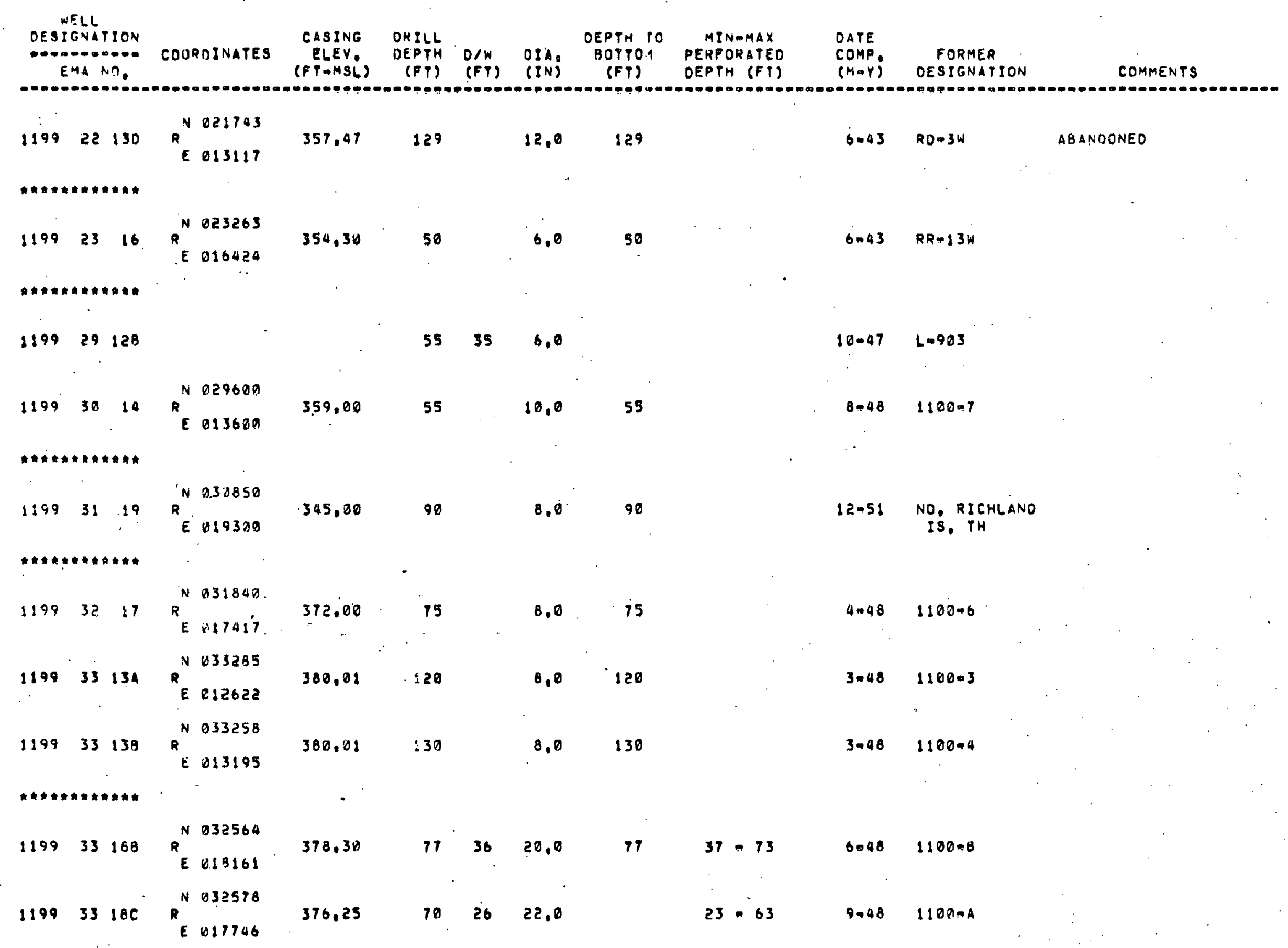


$\because$ WELL

DESIGNATION

tMA NO

COOROINATES

CASING ORILL

FTOMSL.

ORILL
AE.PTH

DEPTH TO

MIN-MAX
PRFORATE

DATE

EMA NO:

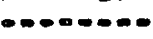

(FT) (FT) (IN) (FY) OEPTH (FT)

( $M \cap Y$ )

FORMER

OESIGNATION

COMMENTS

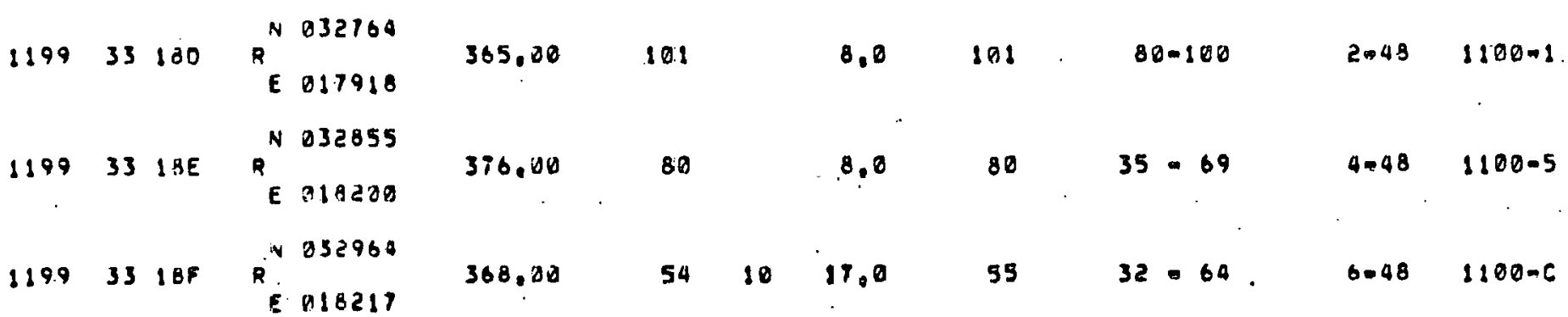

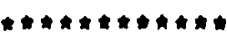

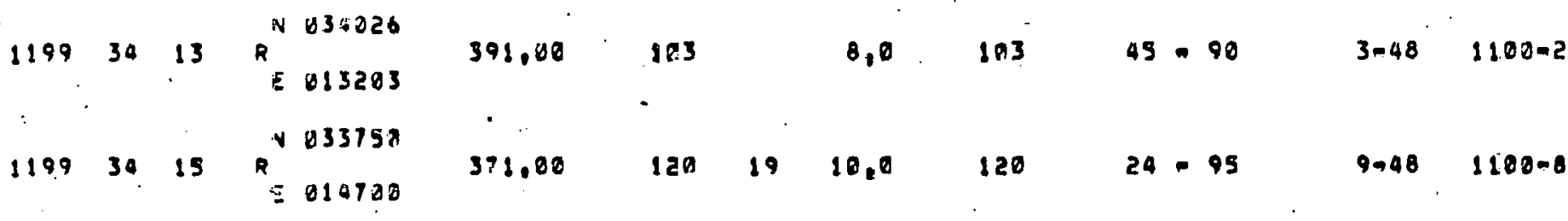

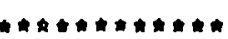

$11993419 \quad R^{N 034260}$

62

$8.0 \quad 63$

$10047 \quad F-10, \times 772$

$1199 \quad 35 \quad 16 \quad Q^{N 0352 \dot{2}} \quad 379.57$

Q. $\quad F=14$

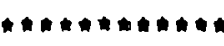

\begin{tabular}{|c|c|c|c|c|c|c|c|c|c|c|c|}
\hline 1899 & 30. & 11 & $R^{N}$ & $\begin{array}{l}937656 \\
01262 i\end{array}$ & 352,26 & 90 & 6,0 & 90 & & 9044 & $3000-0.4$ \\
\hline 1199 & 39 & IAA & $R^{N}$ & $\begin{array}{r}038585 \\
009526\end{array}$ & 357.15 & 100 & 6.0 & 100 & & $9=44$ & 3600-0-3 \\
\hline 1199 & 39 & 168 & $R_{E}^{N}$ & $\begin{array}{l}039072 \\
01916 x\end{array}$ & 352.62 & 54 & 6,0 & 54 & & $8-44$ & $3000-0=2$ \\
\hline 1199 & 39 & 161 & $R^{N}$ & $\begin{array}{l}038831 \\
015984\end{array}$ & 368.82 & 62 & 17,0 & 63 & $22 \cdot 58$ & $8-48$ & $3000=E$ \\
\hline
\end{tabular}




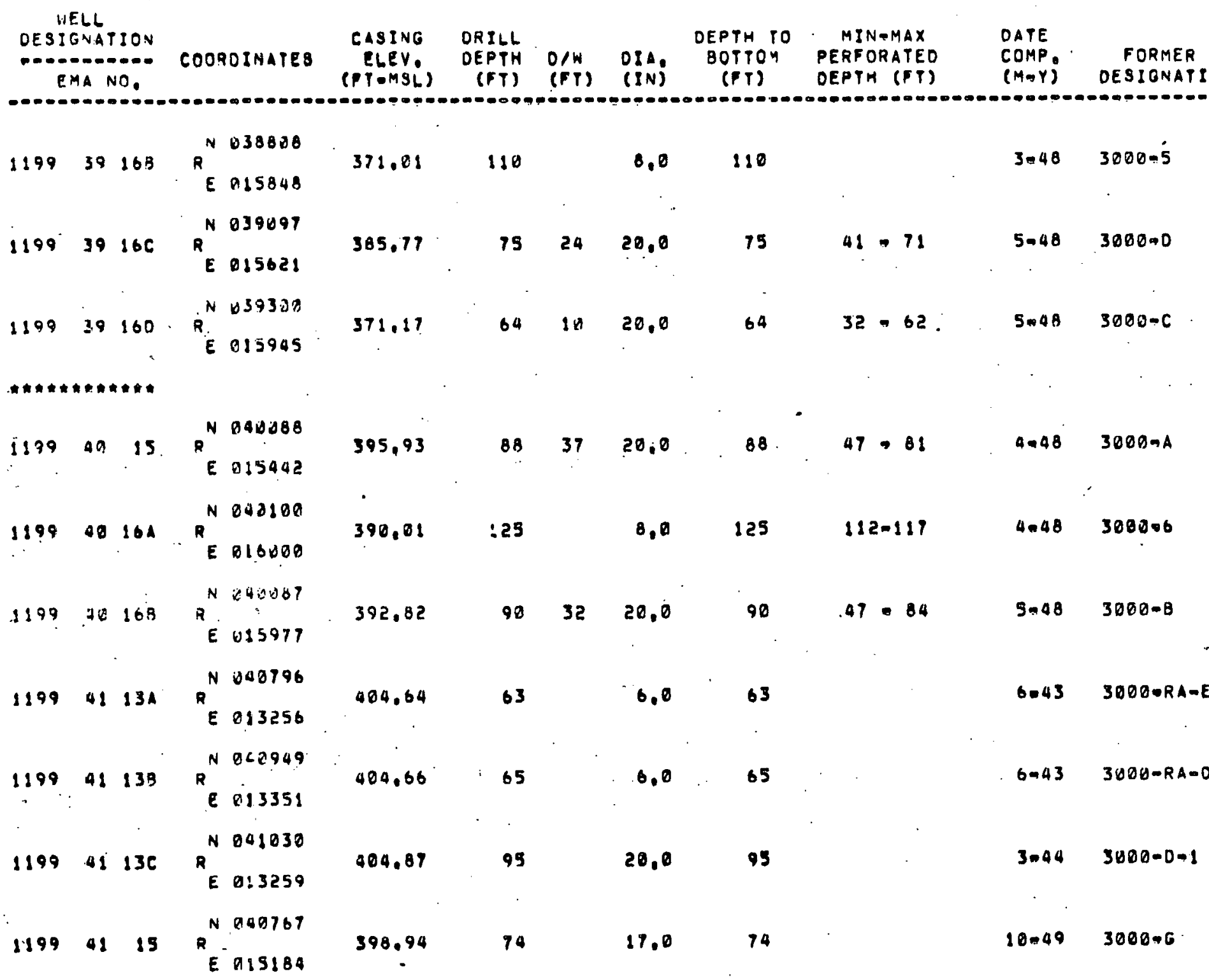


WELL

OESIGINATION

EHA NO.

COORDIVATES

CASINZ̈ ORILL

ORILL DEPH TO
DEPYH O/W OIA. BOTPOM

MINAMAX

PERFORATED

DEPTH (FT)

DATE

(FT-MS) (FT) (FT) (IN) (FT)

$(\mathrm{M}-\mathrm{Y})$

FORMER

DESIGNATION

COMMENTS

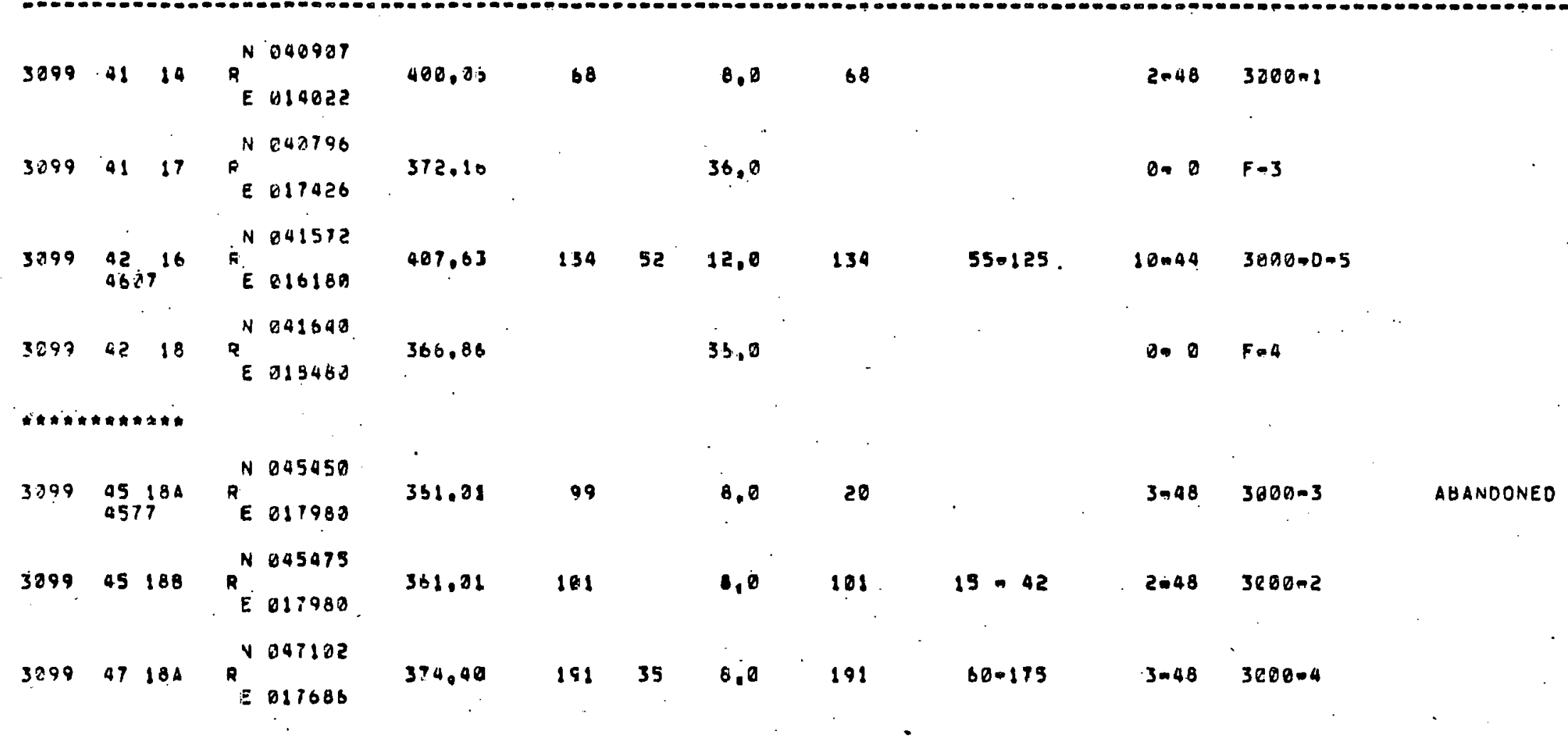


No. of Copies

Offsite

1 ERDA Chicago Patent Office

A. A. Churn

3 ERDA Technical Information Center

On Site Hanford

6 ERDA Richland Operations Office

0. J. Elgert

P. G. Holsted

M. W. Tiernan

J. A. Fernandez

F. C. Robinson

P. G. Rhoades

19 Atlantic Richfield Hanford Company

F. R. Dornheim

R. A. Deju

R. E. Isaacson

L. E. Bruns

W. H. Price

S. M. Price

K. R. Price

K. R. Fecht

D. J. Brown

R. E. Gephart

C. T. Webster

R. A. Arnett

R. K. Ledgerwood

M. Legatski

E. J. Rink

H. P. Shaw

ARHCO File

1 Hanford Environmental Health Foundation

L. J. Maas

$1 \quad$ Vitro/AII

S. S. Compton

1 Westinghouse Hanford Co. - HEDL

R. B. Hall 
3 United Nuclear Inc.

T.E. Dabrowski

W. G. Westover

J. P. Schmidt

33 Battelle-Northwest

D. B. Cearlock

J. R. Eliason

J. R. Raymond

A. E. Reisenauer

V. L. McGhan

E. L. Hilty

P. S. Schrotke

R. W. Wallace

D. W. Damsheu

C. M. Unruh

H. V. Larson

D. A. Myers

J. J. Fix

J. H. Soehnlein

J. P. Corley

P. J. Blumer

M. W. Leale

L. J. Kirby

Technical Publications

Technical Information 


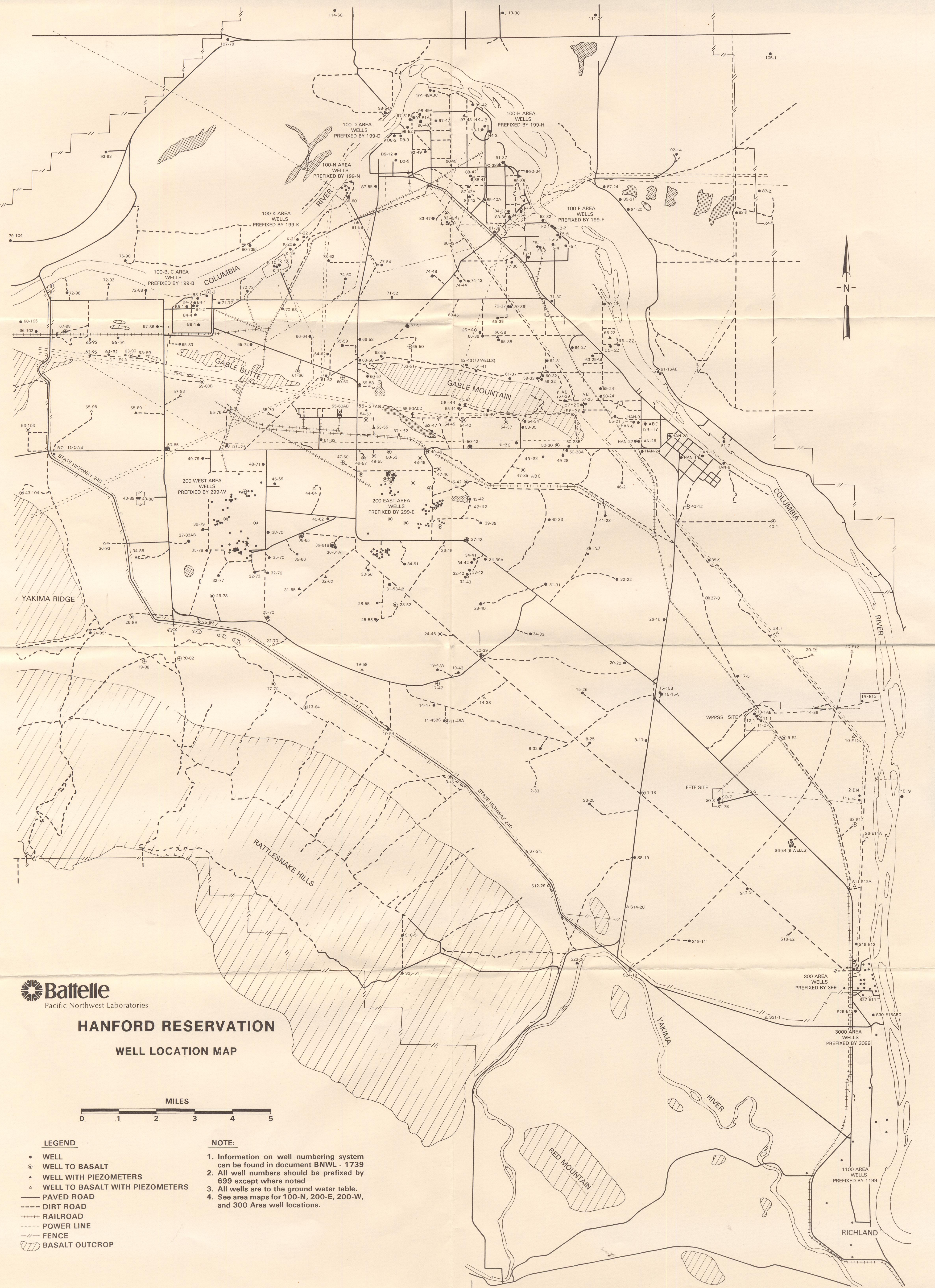

